\title{
Resolve in International Politics
}

\author{
Dissertation
}

Presented in Partial Fulfillment of the Requirements for the Degree Doctor of Philosophy in the Graduate School of The Ohio State University

By

Joshua David Kertzer, B.A.Hons, M.A.

Graduate Program in Political Science

The Ohio State University

2013

Dissertation Committee:

Richard K. Herrmann, Advisor

Bear F. Braumoeller

Kathleen M. McGraw

Christopher F. Gelpi 
(c) Copyright by

Joshua David Kertzer

2013 


\section{Abstract}

Why do some leaders and publics display remarkable persistence in war, while others "cut and run" at the first sign of trouble? Why did the French remain in the First World War despite having suffered nearly a third of a million soldiers killed, missing, or wounded in the Battle of Verdun alone, while the United States immediately halted its military operations in Somalia after 18 of its soldiers were killed during the Battle of Mogadishu? Although resolve is one of the most frequently used independent variables in International Relations, used to explain everything from developments on the battlefield to deliberations at the bargaining table to decisions at the ballot box, we have very little sense of why some actors are more resolved than others. I argue that resolve is an interaction between situational stakes and dispositional traits; by pointing to a series of dispositional characteristics frequently studied in a growing body of research on willpower in behavioral economics and social psychology (time and risk preferences, honor orientations, and trait self-control), I disaggregate the costs of war and explain why certain types of actors are more sensitive to the costs of fighting, while others are more sensitive to the costs of backing down. I test this argument at the micro-level with laboratory and survey experiments, and at the macro-level with Boolean statistical analyses of great power military interventions from 1946-2003. The macro-level analyses suggest that resolve indeed boosts the probability of victory, finding evidence in favor of country-level situational and leader-level dispositional sources of resolve. 


\section{Acknowledgments}

I am indebted to an embarrassingly long list of people for making this project possible.

There is evidently something about supervising this dissertation that compels one to seek out administrative responsibilities, which makes the amount of time that Rick Herrmann, Bear Braumoeller, Kathleen McGraw and Chris Gelpi were able to devote to the project all the more incredible. Rick Herrmann, my advisor, has a nose for the dreaded "so what?" question, introduced me to experiments and behavioral decision-making in IR before they were fashionable, and was willing to give me just enough latitude to develop as a scholar without falling into the abyss; the micro-level side of the project is made in his image. Bear Braumoeller encouraged me to pursue a recklessly counter-cyclical dissertation topic, and is the inspiration behind the macro-level side of the project, as he is for my interests in fast optimization algorithms and slow food. Kathleen McGraw let me act as her apprentice, and taught me everything I know about too many things to name. Chris Gelpi kindly came on board mid-voyage, and the project is all the better thanks to his constructive advice and intellectual generosity.

Beyond my committee, it was in conversations with Alex Wendt about how going to war was like quitting smoking that my interest in resolve took root; without his encouragement to pursue a "big question", the project would have probably been more tractable but less interesting. Luke Keele, Irfan Nooruddin, and Brian

Rathbun were more generous with their time and savoir faire than they had any right to be, and better mentors than anyone could ask for.

Previous versions of this project benefited from quizzical glances and skeptical questions during presentations at Binghamton University, Dartmouth College, 
Harvard University, McGill University, New York University, The Ohio State University, Princeton University, the University of California Santa Barbara, the University of Illinois at Urbana-Champaign, the University of Kansas, the University of South Carolina, the 2011 International Studies Association Meeting in Montreal, the 2012 New Faces in International Security conference at the University of North Carolina at Chapel Hill, and the 2012 Peace Science Society Meeting in Savannah, GA. The survey experiment in Chapter 4 was made possible thanks to the National Science Foundation's Time-sharing Experiments for the Social Sciences (TESS) initiative; I am grateful to Jeremy Freese, Penny Visser, and the anonymous reviewers for their help in improving the experiment's instrumentation, and Ben DiPaola and Poom Nukulkij at GfK for their assistance fielding the study. The project was also supported in part by a doctoral fellowship from the Social Sciences and Humanities Research Council of Canada, and a dissertation-year fellowship from the Graduate School at Ohio State.

For thoughtful feedback or assistance, I am grateful to Scott Althaus, Adam Berinsky, Daniel Bessner, Bill Boettcher, Jake Bowers, Steve Brooks, David Carter, Dave Clark, Mike Colaresi, P.E. Digeser, Peter Feaver, Ben Fordham, Jeff Frieden, Jeff Friedman, Stephen Gent, Mike Horowitz, Nahomi Ichino, Iain Johnston, Katja Kleinberg, Christine Leppard, Rebecca Littman, Amir Lupovici, Melissa Michelson, Neil Narang, Krzysztof Pelc, Paul Poast, Bob Powell, Vincent Pouliot, Patrick Shea, Beth Simmons, Chris Skovron, Harvey Starr, Tricia Sullivan, Dustin Tingley, Chris Twomey, Stephen Weatherford, and Bill Wohlforth, as well as from colleagues at Ohio State, including Bentley Allan, Jan Box-Steffensmeier, Zoltan Buzas, Austin Carson, Aldous Cheung, Raphael Cunha, Chris Devine, Marina Duque, Kevin Duska Jr., Marcus Holmes, Ted Hopf, Jason Keiber, Tim Luecke, Meri Ellen Lynott, Sebastien Mainville, Jennifer Mitzen, Jason Morgan, John Oates, Katy Powers, Xiaoyou Pu, Randy Schweller, Srdjan Vucetic, Alex Thompson, Joshua Wu, and especially Ben Jones and Eleonora Mattiacci, my fellow two musketeers. I'm also thankful to Véronica Betancourt, Ryan Brutger, Jarett Henderson, George Ibrahim, 
Erica Jones, Arpi Kovacs, Francesca McLin, Aaron Moats, Patrick Morrison, Alex Schoenfeld, J. Brendan Shaw, Nick White, and Mike Wilton for putting up with me, and to Pat Clifford, Sharon Friesen, David Haglund, Kim Nossal, Rima BernsMcGown and Barbara Falk for steering me towards this path.

A final thanks goes to my family: Adrienne, Jonathan, and Nicholas Kertzer. If all dissertation topics are autobiographical, it merits noting that my mother once called me while I was at the grocery store to ask why I was getting groceries when I should be working on my dissertation. If resolve indeed has a dispositional component, I'm sure I got it from her. 


\section{Vita}

September 14, 1984 .................. Born in Calgary, AB, Canada

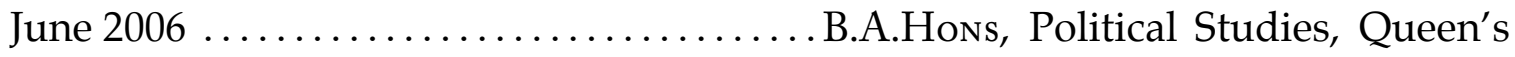
University

December 2007 ......................... Political Science, University of Toronto

December 2009 ......................... Political Science, The Ohio State University

\section{Publications}

Joshua D. Kertzer, "Making Sense of Isolationism: Foreign Policy Mood as a Multilevel Phenomenon," Journal of Politics 75:1 (January 2013), 225-240.

Joshua D. Kertzer and Kathleen M. McGraw, "Folk Realism: Testing the Microfoundations of Realism in Ordinary Citizens," International Studies Quarterly 54:2 (June 2012), 245-258.

David G. Haglund and Joshua D. Kertzer, "From Geo to Neo: A Speculative Inquiry into the Unusual "Geo-Ethnic" Roots of Neoconservatism in U.S. Foreign Policy," Geopolitics 13:3 (2008), 519-544.

Joshua D. Kertzer, "Seriousness, grand strategy, and paradigm shifts in the "war on terror"', International Journal 62:4 (Autumn 2007), 961-979.

\section{Fields of Study}

Major Field: Political Science

International Relations, Political Psychology 


\section{Contents}

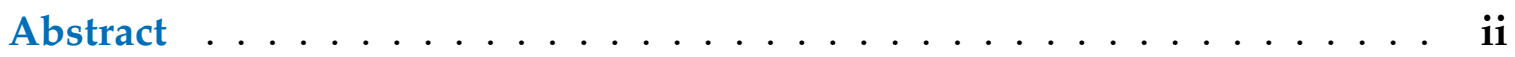

Acknowledgments ................... iii

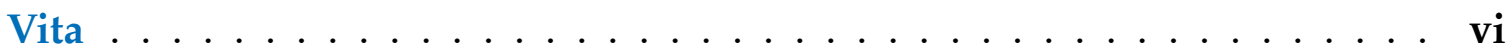

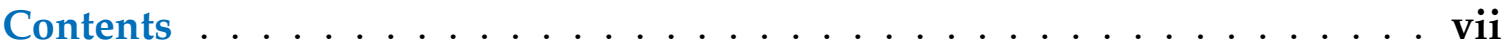

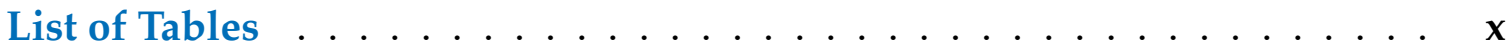

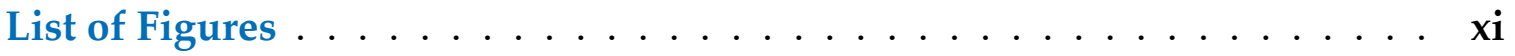

1 Willful Neglect: Resolve in International Politics . . . . . . . . . . . 1

1.1 Defining resolve . . . . . . . . . . . . . . 6

1.2 Resolve in international politics . . . . . . . . . . . 8

1.3 Capabilities, intentions, and resolve . . . . . . . . . . 10

1.4 Two stories about resolve . . . . . . . . . . . . . . . . . . . 15

Situational theories of resolve . . . . . . . . . . . . 16

Dispositional theories of resolve . . . . . . . . . . . . 18

Comparing the two stories . . . . . . . . . . . . . . . 21

The agent-structure problem . . . . . . . . . . . 21

Commitment problems . . . . . . . . . . . . . . 22

Capabilities, intentions, and resolve, redux . . . . . . . . 23

1.5 Conclusion . . . . . . . . . . . . . . . . . . . . . . . 24

2 An Interactionist Theory of Resolve . . . . . . . . . . . . . . . 26

2.1 Three motivations for interactionism . . . . . . . . . . 27

2.2 An interactionist theory of resolve . . . . . . . . . . . 31

Situational determinants of resolve . . . . . . . . . . . . 32

The costs of fighting: casualties . . . . . . . . . . . . 34

The costs of backing down: the issues at stake, and reputation 36

Dispositional and interactionist determinants of resolve . . . . . . 38

Time preferences . . . . . . . . . . . . . . . 39

Risk preferences . . . . . . . . . . . . . . . . . . . . 41

Cultures of honor . . . . . . . . . . . . . . . . . . 42

Trait self-control . . . . . . . . . . . . . . . . . . 44

2.3 Conclusion . . . . . . . . . . . . . . . . . . 46 
3 Experimental Microfoundations for Resolve: I . . . . . . . . . . . . 48

3.1 Research design and materials . . . . . . . . . . . . . . . . 48

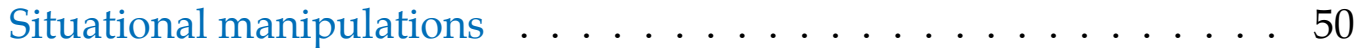

Dispositional measures . . . . . . . . . . . . . . . . . . 51

3.2 Results . . . . . . . . . . . . . . . . 53

How resolved were the participants? . . . . . . . . . . . . . 53

Situational determinants of resolve . . . . . . . . . . . . . . 55

A more precise measure of resolve . . . . . . . . . . 57

3.3 Dispositional determinants of resolve . . . . . . . . . . 63

Interactionist analyses $\ldots \ldots \ldots \ldots \ldots \ldots \ldots$

Time preferences . . . . . . . . . . . . . . 73

Risk preferences . . . . . . . . . . . . . . . . . 75

3.4 Conclusion . . . . . . . . . . . . . . . . . . . . . 77

4 Experimental Microfoundations for Resolve: II . . . . . . . . . . . . . 82

4.1 Materials . . . . . . . . . . . . . . . . 83

Intervention scenario $\ldots \ldots \ldots \ldots$. . . . . . . . . . 83

Dispositional questionnaire . . . . . . . . . . . . 86

4.2 Results . . . . . . . . . . . . . . . . . . . . 89

How resolved were the participants? . . . . . . . . . . . . . . 89

Situational treatments . . . . . . . . . . . . . . . . . 91

Why do casualties fail to affect participants' resolve? . . . . . . . . . 96

Dispositional and demographic results . . . . . . . . . . . . . . . . 99

Interactionist results . . . . . . . . . . . . . . . . . . . 106

4.3 Conclusion . . . . . . . . . . . . . . . . . 107

5 Resolve in Great Power Military Interventions, 1946-2003 . . . . . . . . . 115

5.1 Microfoundations and latent variables . . . . . . . . . . . . . 116

5.2 Methods . . . . . . . . . . . . . . . . . . . . . 121

5.3 Data and variables . . . . . . . . . . . . . . . . 125

Situational variables . . . . . . . . . . . . . . . . . 126

The costs of fighting . . . . . . . . . . . . 126

The costs of backing down . . . . . . . . . . . . . 127

Dispositional variables . . . . . . . . . . . . . . . . . . . . . 129

Time preferences . . . . . . . . . . . . . . . 130

Risk preferences . . . . . . . . . . . . . . . . 130

Cultures of honor . . . . . . . . . . . . . . . . . . 132

Trait self-control . . . . . . . . . . . . . . . . . . . . 133

5.4 Results . . . . . . . . . . . . . . . . . . . . . . . 133

Model selection . . . . . . . . . . . . . . . . . . . . . 133

Resolve: situational and dispositional . . . . . . . . . . . . . 135

Alternative model specifications . . . . . . . . . . . . . . . . . 145

Resolve: situational or dispositional . . . . . . . . . . . . . . . 148

How well do the Boolean models predict conflict outcomes? . . . . . 154 
5.5 Conclusion . . . . . . . . . . . . . . . . . . . 157

6 Conclusion: Solving for $x \ldots \ldots \ldots \ldots \ldots$

6.1 Resolve in other domains . . . . . . . . . . . . . . . . . . . . 169

6.2 How private is private information? . . . . . . . . . . . . . 169

6.3 Resolve and capabilities . . . . . . . . . . . . . . . . . 171

6.4 On the dyadic nature of resolve . . . . . . . . . . . . . . . . 173

6.5 The dark side of resolve . . . . . . . . . . . . . . . . . 175

6.6 On the distinction between situations and dispositions $\ldots \ldots \ldots 178$

6.7 Textual analysis of leaders' speeches . . . . . . . . . . . . . . . . 181

6.8 Against a pure dispositional, and a "garbage can" model of resolve . 183

A Appendix: The Person-Situation Debate . . . . . . . . . . . 186

A.1 Interactionism in action: the person-situation debate . . . . . . . . 186

B Appendix: Lab Experiment Supplemental Analyses . . . . . . . . . . 190

B.1 Manipulation checks . . . . . . . . . . . . . . . . . . . 190

B.2 Demand characteristics . . . . . . . . . . . . . . . . . . . . . . 194

B.3 Scale reliabilities . . . . . . . . . . . . . . . . . . . . . 195

B.4 Demographic controls . . . . . . . . . . . . . . . . . 196

B.5 Survival curves . . . . . . . . . . . . . . . . . . . 197

B.6 Results from fully-crossed factorial analyses . . . . . . . . . . 205

C Appendix: Survey Experiment Supplementary Analyses _ . . . . . . . 207

C.1 Treatment heterogeneity checks . . . . . . . . . . . . . 207

C.2 Estimating the casualty treatment effect for compliers . . . . . . . 208

D Appendix: Boolean Supplementary Analyses . . . . . . . . . . . . . 212

D.1 Alternative bootstrapped boolean coefficient estimates . . . . . . 212

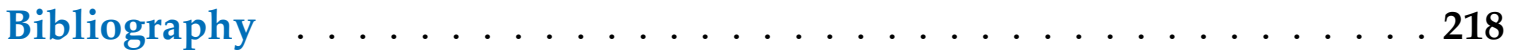




\section{List of Tables}

2.1 Rationales for interactionist theorizing . . . . . . . . . . . 28

3.1 Situational determinants of the duration of the intervention . . . . . . . 56

3.2 Treatment effects based on initial decision to invade . . . . . . . . . 57

3.3 Dispositional and situational determinants of resolve (1) . . . . . . 67

3.4 Dispositional and situational determinants of resolve (2) . . . . . . 68

4.1 Treatment effects based on initial decision to invade . . . . . . . . . . . 91

4.2 Dispositional determinants of resolve . . . . . . . . . . . . . . 101

4.3 Comparison of results across experimental formats . . . . . . . . . . 108

5.1 Model combinations . . . . . . . . . . . . . . . . . . . . . 133

5.2 Boolean model estimates: situation $x$ disposition . . . . . . . . . . . 136

5.3 Situation $x$ disposition alternative model specifications) . . . . . . . . . 144

5.4 Boolean model estimates: situation or disposition . . . . . . . . . . . 149

5.5 Logistic model estimates: situation + disposition . . . . . . . . . . . 156

6.1 Comparison of results: experimental and observational data . . . . . . 164

B.1 Reputation costs . . . . . . . . . . . . . . . . . . . . . . . 193

B.2 Scale reliabilities . . . . . . . . . . . . . . . . . . . . . . . . 196

B.3 Demographic characteristics and resolve . . . . . . . . . . . . 197

B.4 Hazard ratios: full factorial . . . . . . . . . . . . . . . . . . 205

C.1 Casualty treatment effect by age . . . . . . . . . . . . . . . . . 208

C.2 Casualty treatment effect by education . . . . . . . . . . . . . . 208

C.3 Casualty treatment effect by region . . . . . . . . . . . . . . . . . 209

C.4 Casualty treatment effect by income . . . . . . . . . . . . . . . . . 209

C.5 Casualty treatment effect by ethnicity . . . . . . . . . . . . . . 209 


\section{List of Figures}

1.1 Content analysis of Fearon $(1994,1995,1997) \ldots \ldots \ldots \ldots \ldots$

1.2 A spectrum of theories about resolve . . . . . . . . . . . . . . . 15

2.1 Resolve as a dependent variable . . . . . . . . . . . . . . . . 35

3.1 Laboratory experiment structure . . . . . . . . . . . . . . . . . . . . 49

3.2 Distribution of the dependent variable . . . . . . . . . . . . . . 54

3.3 Coefficient plot of situational determinants of resolve . . . . . . . . 58

3.4 Mediation structure . . . . . . . . . . . . . . . . . . . 60

3.5 Nonparametric mediation effects: lab experiment . . . . . . . . . . . 62

3.6 Quadratic effect of risk aversion . . . . . . . . . . . . . . . . . . 69

3.7 Coefficient plot of situational and dispositional correlates of resolve . . . 73

3.8 Time preferences $x$ casualties combined coefficient plot . . . . . . . . 74

3.9 Risk preferences $x$ reputation cost combined coefficient plot . . . . . . 76

3.10 Risk preferences $x$ casualty combined coefficient plot . . . . . . . . . 77

4.1 Survey experiment structure . . . . . . . . . . . . . . . . . . 85

4.2 Distribution of dependent variable . . . . . . . . . . . . . . 90

4.3 Coefficient plot of situational determinants of resolve . . . . . . . . . 92

4.4 Nonparametric mediation effects: survey experiment . . . . . . . . . . 95

4.5 Bootstrapped mean casualty predictions by experiment format . . . . . 998

4.6 Coefficient plot of situational and dispositional correlates of resolve (1) . 102

4.7 Quadratic effect of risk aversion . . . . . . . . . . . . . . . . . . 103

4.8 Coefficient plot of situational and dispositional correlates of resolve (2) . 105

4.9 Risk orientation $x$ reputation costs combined coefficient plot . . . . . . 112

4.10 Honor culture $\mathrm{x}$ anticipated costs combined coefficient plot . . . . . . . . 113

4.11 Smoking behavior $x$ casualties combined coefficient plot . . . . . . . . 114

5.1 Decision tree for studying resolve with observational data . . . . . . 119

5.2 Bootstrapped boolean coefficient estimates: model 1 from Table 5.2 . . 137

5.3 Bootstrapped boolean coefficient estimates: model 2 from Table 5.2 . . 138

5.4 Bootstrapped boolean coefficient estimates: model 3 from Table 5.2 . . 139

5.5 Bootstrapped boolean coefficient estimates: model 4 from Table 5.2 . . 140

5.6 Fitted values: conjunctural causation . . . . . . . . . . . . . . . . 145

5.7 Bootstrapped boolean coefficient estimates: model 1 from Table 5.4 . . 150

5.8 Bootstrapped boolean coefficient estimates: model 2 from Table 5.4 . . . 151

5.9 Fitted values: substitutable causation . . . . . . . . . . . . . . . . . 153 
5.10 ROC curves for evaluating model predictive power . . . . . . . . . 155

5.11 Separation plots show the predictive superiority of the Boolean models . 158

A.1 Resolve as probability density functions . . . . . . . . . . . . . . 187

B.1 Bootstrapped mean casualty prediction errors . . . . . . . . . . . . . 195

B.2 Situational survival curves . . . . . . . . . . . . . . . . . . . . . 199

B.3 Dispositional survival curves . . . . . . . . . . . . . . . . 200

B.4 Nonlinear effect of risk preferences survival curves . . . . . . . . . . 201

B.5 Time preferences $x$ casualty survival curves . . . . . . . . . . . . 202

B.6 Risk preferences x reputation survival curves . . . . . . . . . . . . . 203

B.7 Risk preferences x casualty survival curves . . . . . . . . . . . . . . 204

B.8 Hazard ratio plots: full factorial analysis . . . . . . . . . . . . . . . 206

C.1 Casualty treatment p-values for compliers . . . . . . . . . . . . . . 210

D.1 Bootstrapped boolean coefficient estimates: model 1 from Table 5.3 . . . 213

D.2 Bootstrapped boolean coefficient estimates: model 2 from Table 5.3 . . . 214

D.3 Bootstrapped boolean coefficient estimates: model 3 from Table 5.3 . . . 215

D.4 Bootstrapped boolean coefficient estimates: model 4 from Table 5.3 . . . 216

D.5 Bootstrapped boolean coefficient estimates: model 5 from Table 5.3 . . . 217 


\section{Chapter 1: Willful Neglect: Resolve in International Politics}

“Desire, wish, will, are states of mind which everyone knows, and which no definition can make plainer."

-William James (1950 [1890], p. 486)

On January 12, 2010, an earthquake struck the island nation of Haiti, reducing much of the capital city of Port-au-Prince to rubble. In the days afterward, as the casualty estimates grew by the hundreds of thousands and the international community turned its attention towards rebuilding the ravaged country, pundits pontificated on the uphill battle faced by a country that had suffered as many man-made disasters as natural ones. Bob Herbert, writing in the New York Times, struck an optimistic note: the Haitians would succeed, he argued, because they had shown "resolve among the ruins." (Herbert, 2010)

Herbert is not alone in positing resolve and its synonyms - willpower, selfcontrol, dedication, tenacity, motivation, determination, drive, and so on - as a solution to political problems. The collapse of the Mubarak regime in Egypt has frequently been attributed to the resolve of the protesters in Tahrir Square ("their determination is unshakeable," noted the editor of the Egyptian Daily News (Michaels and May, 2011)), while the same attribute has been used to account for the opposite event in neighboring Libya, where Moammar Qadaffi clung to power despite months of NATO airstrikes and armed insurrection ("War is largely about willpower," wrote an analyst for the DC-based Washington Institute for Near East Policy, “and Qadaffi currently holds the upper hand on this front."(White, 2011). Writing a year prior in The Weekly Standard, Charles Krauthammer dismissed prophe- 
cies of American decline (e.g. Kennedy, 1987; Zakaria, 2008) by arguing that "decline is a choice" rather than "a condition," and that the slide towards multipolarity can therefore be reversed through "moral self-confidence and will." (Krauthammer, 2009). ${ }^{1}$ Similarly, when the Canadian government was deliberating over whether to renew its deployment in Afghanistan in 2008, the independent panel it convened to issue recommendations released a report arguing that Canadians must exhibit "sustained resolve" in order to allow the mission to succeed (Manley et al., 2008, 8). What makes these arguments interesting is not that they posit resolve as a solution to personal problems - motivational speakers and authors of self-improvement books have made millions telling us that already — but that they frame it as a solution to political problems.

After all, given the vast chasm separating the pages of International Organization from the oeuvre of Tony Robbins, it is not clear that scholars of the self-help system have that much to learn from the other kind of self-help books. Yet nowhere is this embrace of resolve more evident than in the study and practice of war. Although Blainey $(1973,122)$ suggested that "wars usually begin when fighting nations disagree on their relative strength," many military practitioners emphasize not bouts of strength, but feats of will. Napoleon Bonaparte famously declared that "in war, the moral is to the physical as three to one" (Chandler, 1966, 155), while Marshall Foch, the commander of the French Ninth Army at the Battle of the Marne in 1914, went even further, proclaiming in his lectures at the École de Guerre that "in a material sense no battle can be lost" (Recouly, 1920). This belief in the power of resolve formed a major part of the "cult of the offensive" that reigned supreme in military academies across Europe by the time of the First World War (Van Evera, 1984, 61): pre-war British training manuals announced that that "moral force in modern war preponderates over physical force" (Travers, 1979, 274), while in Germany in 1916, Paul von Hinderberg, then Chief of the German General Staff, declared that "vic-

\footnotetext{
${ }^{1}$ The article, influential in conservative quarters, was later cited by the Republican Congressman (and later, Vice Presidential nominee) Paul Ryan in his June 2, 2011 speech to the Alexander Hamilton Society (Warren, 2011).
} 
tory will go to him who has the best nerves" (Watson, 2008, 1).

Indeed, resolve is a ubiquitous ingredient in International Relations (IR) theory, used to explain everything from why states win wars (Maoz, 1983; Morrow, 1988; Pape, 1996), to how they prevail during crisis bargaining, thereby preventing conflict from breaking out (Schelling, 1966; Snyder and Diesing, 1977; Morrow, 1985; George and Simons, 1994; Lebow, 1998). Rationalist approaches to the study of international conflict revolve around resolve: if both sides are aware of each others' levels of motivation, the less resolute side backs down before a crisis can even take place (Powell, 1987; Morrow, 1989; Fearon, 1994, 1995). Likewise, it is motivation, not muscle, that is used to explain why great powers fare so poorly in asymmetric conflict (Mack, 1974), and why the United States was never able to push the North Vietnamese to their "breaking point" during the Vietnam War (Betts, 1980; Mueller, 1980).

Despite this ubiquity, I argue that IR scholars have vastly undertheorized resolve: although the concept drives many of our theories of world politics, our understanding of the concept is marred by three lacunae. First, we have little sense of what resolve is conceptually - how, for example, it differs from capabilities and intentions. Second, resolve is unobservable, but because we do not agree on what it is, we cannot agree on how to measure it, which makes it difficult to subject our theories about resolve's effects to rigorous empirical testing. Third, where does resolve come from? Although we often explain behavior and outcomes in IR by pointing to variation in actors' levels of resolve, we have trouble explaining this variation itself. In short, we lack microfoundations, and with them, a theory of resolve.

In this dissertation I attempt to rectify this neglect, connecting work on resolve in IR with an emerging body of research on willpower and self-control elsewhere in the social sciences to propose an "interactionist" theory of resolve that envisions it to be a function of both dispositional and situational causes, both traits and stakes. I then test the theory at two levels of analysis, studying resolve first as explanan- 
dum and then as explanans. At the individual level, I employ a series of original laboratory and embedded survey experiments to probe the microfoundations of resolve in the context of military interventions. I then use these experimental findings to build composite situational and dispositional measures of resolve with observational data at the leader- and country-level, enabling me to test whether resolved great powers were indeed more likely to prevail in military interventions from 1945-2003.

As as Sartori $(1970,1038)$ famously argued, "concept formation stands prior to quantification," so before proposing or testing a theory of resolve, it is important to clearly specify why we need one in the first place, and discuss the problems confronting the field. Thus, in this first chapter, I begin by defining resolve and reviewing the manner in which it has been used in IR, arguing that we have built many of our theories of world politics around a phenomenon that has been inconsistently theorized. I then connect the IR literature to a growing body of work on willpower from elsewhere in the social sciences to suggest that there have typically been two types of "stories" social scientists tell about resolve: an inside, dispositional account that understands resolve to be a kind of trait, and an outside, situational one that perceives resolve to be a function of the costs or stakes an actor faces.

In Chapter 2, I suggest that neither dispositional nor situational theories of resolve are satisfactory by themselves, and that taking resolve seriously requires us to think about the concept in an interactionist manner that connects the two. After discussing the merits of interactionist theories, I propose three dispositional variables (time preferences, risk preferences, and cultures of honor) and two situational variables (casualties, and the costs of backing down), interactions between which can explain why certain types of costs of war loom larger for certain types of actors.

In Chapter 3, I conduct an initial test of the individual-level microfoundations of resolve, employing a novel laboratory experiment that models both the selection into, and duration of support for, military interventions. The experiment manipulates situational features of the military intervention while measuring dispo- 
sitional variables using techniques developed in experimental work in behavioral economics and social psychology. Because of its two-stage structure, I can measure resolve cleanly as a dependent variable by focusing specifically on the extent to which participants who originally supported an intervention continue to do so in the face of mounting costs. In Chapter 4, I further extend the experimental findings, employing a national survey experiment that borrows many of the features of the laboratory experiment while also modifying others to determine the robustness of the results on a nationally representative sample. The experimental analyses offer further evidence of the interactionist nature of resolve.

In Chapter 5, I switch the focus of the analyses. The longstanding obstacle to studying resolve with observational data is that resolve is unobservable; political scientists have thus largely been unable to adequately test whether resolve has the effects we often claim, because we have been forced to infer resolve from the outcomes we also use it to explain. I propose a novel solution: rather than infer resolve via its consequences, we study resolve via its causes. Having already established evidence for situational and dispositional determinants of resolve with experimental data at the individual-level, I use these same variables with observational data at the country- and leader-level to construct composite situational and dispositional measures of resolve for the great powers in the post-WWII era. I then employ Boolean statistics (Braumoeller, 2003) to study the impact of situational and dispositional pathways of resolve on the probability of victory of great power military interventions from 1946-2003. These Boolean analyses with resolve as an explanatory variable allow me to test whether resolve has the positive effects on conflict outcomes that military practitioners and IR scholars often claim, but doing so using measures of resolve derived independently of the behavior and outcomes I am using them to explain. The results suggest that the situational and dispositional determinants of resolve also predict the probability of victory, but at different levels of analysis: conflict outcomes appear to primarily be a function of situational determinants of resolve at the country-level, and dispositional determi- 
nants of resolve at the leader-level. Finally, Chapter 6 concludes by examining the ramifications of the findings, paving the way forward for future analyses.

\subsection{Defining resolve}

Any study of a phenomenon has to begin with a definition. I follow the Oxford English Dictionary in defining resolve as "firmness or steadfastness of purpose," maintaining a policy despite contrary inclinations or temptations to back down. In its emphasis on determined, sustained effort despite temptations to the contrary, resolve can therefore be considered synonymous with willpower, as well as related forms of self-regulation like self-control (the deliberate use of willpower to avoid undesirable actions) and self-discipline (the deliberate use of willpower to achieve desirable goals), and the opposite of weakness of will, irresolution, incontinent action, and other such antonyms.

Defining resolve in this manner has two advantages. First, it reflects the way we use these words in ordinary language: to describe an actor as "resolute" intuitively means the same thing as to argue the actor is persistent, motivated, committed, determined, and so on. Indeed, in his discussion of resolve, O'Neill $(1999,107)$ notes that scholars seem to use a wide variety of terms, which "appear to be manifestations of one unnamed central quality."

Second, it allows the analysis that follows to be grounded in an interdisciplinary fashion. The past several decades have witnessed a Risorgimento of resolve throughout the social sciences, most notably in the psychological literature on self-regulation and the economic literature on intertemporal choice and self-command, but also in fields as varied as the philosophy of action, education research, law and public policy, organizational theory, criminology, and even animal psychology. ${ }^{2}$

\footnotetext{
${ }^{2}$ On self-regulation in psychology, see: Baumeister and Vohs 2004; Moller, Deci, and Ryan 2006; Ryan and Deci 2006; Baumeister, Vohs, and Tice 2007; Galliot and Baumeister 2007; Galliot et al. 2007; Tice et al. 2007; Fritsche, Jonas, and Fankhänel 2008; Ackerman et al. 2009; Finkel et al. 2009; Morsella, Bargh, and Gollwitzer 2009. For economists' growing interest in self-control and intertemporal choice, see: Thaler and Shefrin 1981; Schelling 1984; Loewenstein and Elster 1992; Jolls, Sunstein, and Thaler 1998; Gul and Pesendorfer 2001; Bénabou and Tirole 2002, 2003, 2004; Benhabib
} 
This growth has occurred in tandem despite each literature retaining its own nomenclature: IR theorists tend to favor "resolve," social psychologists typically adopt "self-regulation," economists choose "self-command", criminologists refer to "self-control," social workers prefer "resilience", and philosophers are more likely to endorse "willpower." Similarly, each discipline has predominantly applied the concept to different domains: political scientists frequently restrict resolve to military contexts by defining the term as "willingness to fight" (e.g. Sartori, 2002), whereas economists like Ameriks et al. (2007) tend to define self-control purely in terms of consumption decisions, and developmental psychologists and criminologists focus on self-control in the realm of refraining from socially undesirable actions (e.g. Finkenauer, Engels, and Baumeister, 2005). Despite these differences, however, if theorizing is about following Rosenau (1980) in asking "of what is this an instance?", I argue that the underlying construct remains the same in all cases: a resolute actor is engaged in what Young $(1968,33)$ called "an intensity of feeling": a firmness or steadfastness of purpose, resisting temptations to the contrary. The content of that purpose, then - whether the commitment to go to war, the motivation to quit smoking, or the determination to allocate a certain portion of your income to investment rather than consumption - is secondary to the steadfastness, dogged persistence, or "sticktoitiveness" with which it is being pursued. ${ }^{3}$

and Bisin 2005; Fudenberg and Levine 2006; Read 2006; Ameriks et al. 2007. Philosophers of action have long been interested in weakness of will or akrasia; for recent developments in the literature, see: Holton 1999; Stroud and Tappolet 2003; Hoffmann 2008; May and Holton 2012. Beyond these approaches, we see similar interest in willpower in education research (Corno, 1993; Kohn, 2008), law and public policy (Posner, 1997; Fennell, 2009), (Hecht et al., 2002), criminology (Gottfredson and Hirschi, 1990; Grasmick et al., 1993; Tittle, Ward, and Grasmick, 2003; Pratt, Turner, and Piquero, 2004; Tittle, Ward, and Grasmick, 2004; Wikstrom and Treiber, 2007; Beaver et al., 2010; Boutwell and Beaver, 2010), animal psychology (Tobin and Logue, 1994; Cheng et al., 2002; Miller et al., 2010), as well as work on hardiness and resilience in military psychology (King et al., 1998; Bartone, 2006; Johnsen et al., 2009) and gerontology (Ong et al., 2006; Trivedi, Bosworth, and Jackson, 2011), and a fertile interdisciplinary literature operating at the nexus of multiple fields (Ainslie, 1986; Logue, 1988; Ainslie, 1992; Elster, 2000; Ainslie, 2001; Kalis et al., 2008).

${ }^{3}$ Because of the association between self-mastery and virtue in classical philosophy (Baumeister and Exline, 1999) the philosophy of action literature has traditionally viewed resolve in the context of upholding better judgments (Dunn, 1987), but if resolve merely refers to a firmness of purpose irrespective of its content, one can be as resolved to indulge as one is to abstain (Kivetz and Simonson 2002, see also Rosen 2005, 154). For more on this point, see the discussion of the "dark side of resolve" in Chapter 6. 


\subsection{Resolve in international politics}

Resolve thus defined is a frequent protagonist in IR theory, arising in a wide variety of camps. The first is deterrence theory, whose advocates have long argued that it is essential for states to maintain "reputations for resolve" so that other actors will not try to take advantage of them (Jervis, 1976, 1979; Powell, 2003; Lupovici, 2010). While this line of argument has come under increased scrutiny since actors may not have as much control over their reputations as they think (Mercer, 1996; Tang, 2005), its critics do not downplay the importance of perceptions of resolve so much as suggest that these perceptions are usually outside one's control. ${ }^{4}$ The perception of resolve is an "emotional belief" (Mercer, 2010), such that there may be little that the United States can do to convince al Qaeda that it is not a "paper tiger" (Shannon and Dennis, 2007); for the same reason, despite the fears of US policymakers, American defeats in the developing world during the Cold War did little to weaken Soviet perceptions of American determination (Hopf, 1991b).

The second camp is the crisis bargaining literature more broadly, which argues that states embroiled in crisis negotiations are partaking in games of risk that are won not by strength, but by "nerve" (Schelling, 1960; Iklé, 1964; Schelling, 1966; Young, 1968; Snyder and Diesing, 1977). Crisis bargaining models rely on the same payoff structure as the game of Chicken, a "dilemma of common aversion" (Stein, 1993) where each side prefers to stand firm and induce its opponent to back down, but also prefers retreating over a situation where neither side relents and war emerges. Since bargaining is a competition in risk-taking, an actor that is able to credibly signal its resolve - usually by taking risks that an irresolute actor would be unwilling to tolerate - will successfully avert war on its desired terms (Jervis, 1972; Morrow, 1985; Powell, 1987; Morgan, 1990). Since, however, resolve is an actor's "private information," and there are incentives to misrepresent resolve

\footnotetext{
${ }^{4}$ For a potential exception, see Press (2005), although even here, the "current calculus" theory of credibility argues not that perceptions of resolve are irrelevant, but rather, that they are more likely to stem from what I call situational rather than dispositional factors (e.g. present interests at stake matter more than past behavior).
} 
in order to secure advantages in negotiations (Fearon, 1995), actors often have to rely on commitment mechanisms (Becker, 1961; Elster, 1979, 2000) such as domestic audience costs (Fearon, 1994; Baum, 2004; Slantchev, 2006; Tomz, 2007; Weeks, 2008; Snyder and Borghard, 2011) to signal their resolve. Thus, although rationalist models typically invoke incomplete information and commitment problems as two distinct causes of conflict - the former to be rectified by signaling (sinking costs), the latter via commitment devices (tying hands) (Fearon 1997, although see Slantchev 2005) - resolve figures prominently in both camps, not only because commitment devices are often used to draw inferences about resolve, but because, as will be argued below, it is precisely because of weakness of will that many commitment problems arise in the first place.

In the third camp, international security scholars argue that resolve explains military outcomes. ${ }^{5}$ Writing in the shadow of the Vietnam War, Rosen (1972) claims that to understand which side wins the war, we need to pay attention to each party's "willingness to suffer", and Mueller (1980) points to the importance of the "breaking point" in determining who accepts defeat. Similarly, Maoz (1983) finds that a "balance of resolve" model better explains outcomes of military interstate disputes than a balance of capabilities model does, while Stam (1996) points to several domestic political factors that affect each side's sensitivity to costs and lower the war's net benefits, thereby making belligerents less resolute and more willing to settle for a draw. Just as crisis bargaining theorists argue that war can be prevented if the parties recognize the true balance of motivation (George and Simons, 1994), military scholars suggest that wars will end once the combatants learn each others' levels of resolve (Goemans, 2000), frequently inferred via events on the battlefield (Smith and Stam, 2004; Ramsay, 2008). Resolve affects the outcomes of conflict mediation as well, as third parties can prevent conflict from breaking out either by

\footnotetext{
${ }^{5}$ Recent rationalist scholars of conflict - what Powell $(2004,345)$ calls "the second wave of formal work on war" would reject this distinction between crisis bargaining models and models of military outcomes, envisioning war not as something that happens when bargaining fails, but rather, as a continuation of the bargaining process (Goemans, 2000; Wagner, 2000; Filson and Werner, 2002; Smith and Stam, 2004).
} 
making their own resolve as a mediator known (Walter, 1997, 340-1), or by providing information about the resolve of one of the potential belligerents (Kydd, 2003; Rauchhaus, 2006).

Finally, theories of conflict also give resolve a role at lower levels of analysis by examining the resolve of military units and the public at large. Reiter and Stam (1998a), for example, find that levels of troop morale are positively correlated with military victory, similar to classic findings in military sociology (Shils and Janowitz, 1948; Baynes, 1967). Similarly, although Schultz $(1998,1999)$ argues democracies are often able to avoid war because they are better at signaling their resolve, scholars of counterinsurgencies and asymmetric conflict frequently inquire as to whether democratic publics are too "soft" (Pape, 2003, 349), or cost-intolerant to be resolute (Mueller, 1971; Mack, 1974; Gartzke, 2001; Merom, 2003; Mueller, 2005; Gelpi, Feaver, and Reifler, 2009; Caverley, 2009/10; Lyall, 2010), and so are doomed to suffer what Morgenthau $(1951,222)$ called a "paralysis of will." Even if the public is not casualty-phobic (Gartner and Segura, 1998; Gelpi, Feaver, and Reifler, 2005/06; Gelpi, Reifler, and Feaver, 2007), what makes the "rally around the flag" effect significant is that rallies end just as quickly as they begin (Baum, 2002; Kam and Ramos, 2008), and support for conflict decays over time as the public "loses heart" (Reiter and Stam, 2002, ch. 7, Sullivan, 2008; Fletcher, Bastedo, and Hove, 2009). Beyond the study of war, scholars of governance and statebuilding are increasingly interested in "resilience", the extent to which communities and institutions are able to "bounce back" in response to stresses and risks (Joseph, 2013; Chandler, 2013) - a concept I set aside for most of the discussions that follow, but to which I return in Chapter 6.

\subsection{Conceptual ambiguity: capabilities, intentions, and resolve}

As the above discussion illustrates, references to resolve permeate the international security literature, and are especially prominent in rationalist models of conflict. Indeed, a content analysis of three of the most central pieces by James Fearon from the 
mid-1990s (Fearon, 1994, 1995, 1997) finds that resolve and willingness are invoked nearly 170 times, making it the sixth-most popular term across the three articles, as shown in Figure 1.1.6

Figure 1.1: Content analysis of Fearon $(1994,1995,1997)$

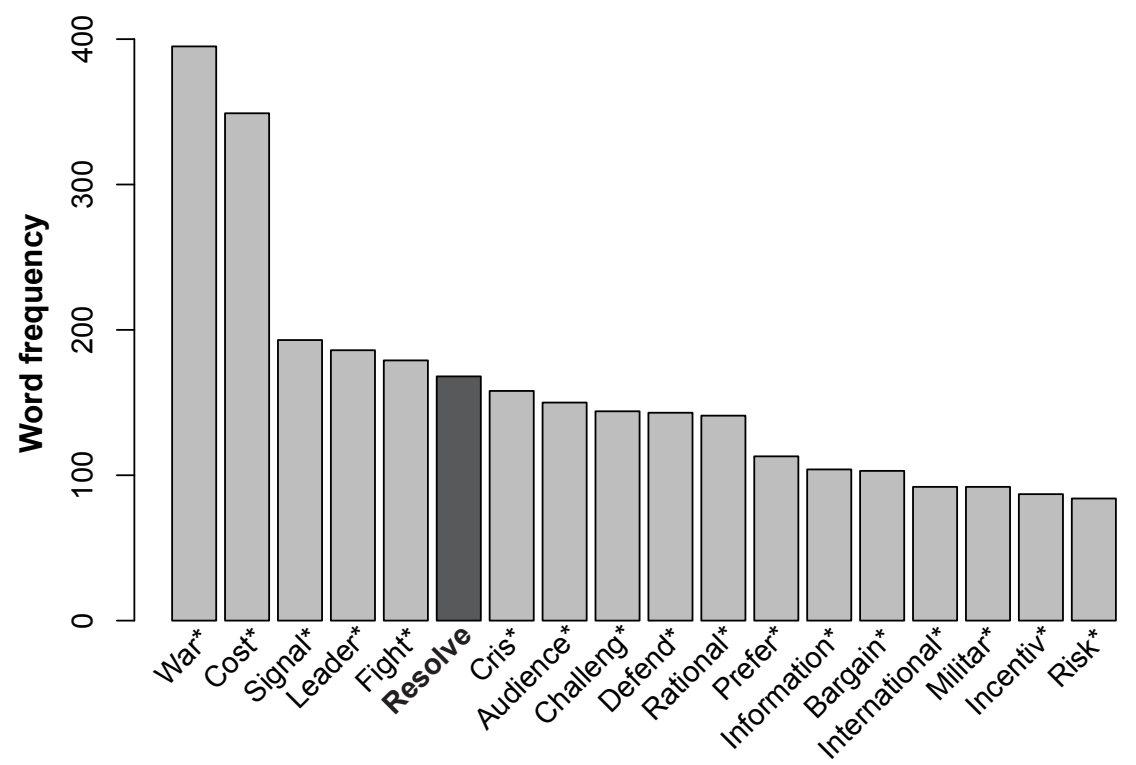

A content analysis of Fearon $(1994,1995,1997)$ finds that resolve and willingness are used nearly 170 times across the three works, making them the sixth-most popular term. As per text mining conventions, the asterisks denote affixes following each word stem: thus, war/wars is most commonly used, followed by cost/ costs / costly / costless, signal/signals / signaling, and so on. Resolve is invoked 1.63 times more than bargaining, 4.67 times more than capabilities, and 5.42 times more than anarchy. Analyses conducted using the tm package in R (Feinerer, Hornik, and Meyer, 2008).

However, despite the popularity of the concept, it is not clear that rationalist models of war in fact need resolve at all, not just because many incomplete information models of war do not directly model resolve (Filson and Werner, 2002; Fey and Ramsay, 2007; Filson and Werner, 2007a), ${ }^{7}$ but also because, as Sartori (2005,

\footnotetext{
${ }^{6}$ As of June 11, 2013, these three articles have been cited over 3800 times according to Google Scholar; it is therefore not surprising that resolve looms so large in our understanding of conflict!

${ }^{7}$ These works are notable not because they are bargaining models of war that do not invoke resolve, but because they are incomplete information bargaining models of war that make no direct mention of the concept. In a complete information game, uncertainty about the other player's level
} 
7) notes, the term has been used in a variety of different ways by different scholars, such that it is unclear that many of the models that invoke resolve are in fact referring to the concept as properly understood.

Realist approaches to international security often argue that states have three pieces of private information: capabilities, intentions, and resolve (Tang, 2008), yet resolve is often presented in a manner that renders it analytically indistinct from the other two, a definitional ambiguity that is all the more surprising given that capabilities and intentions are not conceptually close to one another. ${ }^{8}$ Some theorists lump in resolve with capabilities, an approach that comes in three variants. First are those who view resolve as a source of capability - for example, the classic IR theorists who wrote about "national morale" or "national will" as a component of power (Wright, 1964; Organski, 1968; Cline, 1975; Morgenthau, 1985; Cline, 1994; for a more recent argument about resolve as a source of power, see Reed, 2003). Second are those who invert the relationship, and propose that capabilities are a source of resolve (Snyder and Diesing, 1977; Morrow, 1989; Meirowitz and Sartori, 2008, 329 , fn. 4). These two approaches are less definitional than causal, although the fact that the causal arrow points in different directions in each approach invites further attention towards resolve's relationship with capabilities, causal or otherwise. ${ }^{9} \mathrm{Fi}$ -

of resolve cannot be a cause of war - Fearon $(1994,583)$ shows that when states have complete information about resolve, no foreign policy crisis occurs - so it is therefore unsurprising that models of bargaining in war that do not rely on incomplete information (e.g. Wagner, 2000) would have no need for resolve. It should be noted as well that while these models do not feature references to resolve, they do model the cost of war - a concept some rationalists treat as equivalent to resolve, but which I argue below is distinct.

${ }^{8}$ Capabilities refer to the material resources - military might, a population base, territory, economic clout, and so on - from which an actor's relative power is drawn. Intentions, in contrast, refer to an actor's goals and desires, and therefore, govern how capabilities are used: whether a state is revisionist or status-quo seeking (Schweller, 1994), for example. Thus, while capabilities dictate what an actor can do - you cannot fight wars if you cannot afford to pay for them (Kennedy, 1987) - intentions refer to what an actor wants. That said, although these attributes are conceptually distinct, folk psychologists note that we frequently draw inferences about one of these attributes based on our perceptions of the others. For example, if we know a task is difficult and that someone's capabilities are low, we assume that their resolve is high; likewise, if we see actors give up easily, we not only make inferences about their lack of resolve, but also revise our initial assumptions about their intentions (See Heider, 1958, ch. 4).

${ }^{9}$ For example, it is also possible that the two properties are in a constitutive relationship. On causation and constitution, see Wendt (1998). 
nally, another common approach is to use the terms indistinguishably: Slantchev's (2010) argument about when strong states will feign weakness treats resolve and strength interchangeably (a resolute state is a "strong type"), while Smith and Stam (2004) discuss Saddam Hussein's view of the US public as casualty sensitive during the Gulf War as an instance of "Iraqi beliefs about U.S. capabilities" (p. 808, emphasis added). The idea of willpower similarly captures the extent to which resolve and capabilities are often seen as intertwined.

For others, resolve is used synonymously with aims or intentions: Jervis (1976, 48) suggests the two cannot be separated from one another, and Fearon (1994) uses the terms interchangeably, referring to audience costs both as a device for signaling resolve (e.g. p. 578), and as a tool for signaling intentions (e.g. p. 587), as does Rosato (2003, 598-9), and Trager (2011, 469-72). Both Schultz (1999, 327), Walter $(1997,341)$, and Snyder and Borghard $(2011,3)$ treat resolve as synonymous with "preferences," and although Starr's (1978) notion of "willingness" has been operationalized any number of ways (e.g. Most and Starr, 1989; Gartzke, 1998; Furlong, Gleditsch, and Hegre, 2006), it tends to be treated less about will and more about intentions or preferences, a distinction similarly blurred by Morrow (1988), who makes an offhand reference to "national preferences (i.e. the political will)" (p. 82). Indeed, uncertainty about an actor's resolve is often framed as ambiguous or misperceived intentions: whether Berlin knew if St. Petersburg wanted war or not in 1914 (Copeland, 2000, 90-1), the veracity of Saddam Hussein's assumption that the United States would stand idly by during the 1990 Iraqi invasion of Kuwait (Mearsheimer, 2001, 38), the US trying to convince Argentina that Great Britain's threats of war in the Falklands Crisis weren't bluffs (Kydd, 2003), and so on.

Ultimately, if resolve is the same as intentions or capabilities, it is unclear why we should go to the trouble of using the word "resolve" in the first place. Smith and Stam's (2004) treatment of resolve is illustrative. In their model — based on an earlier approach used in Smith (1998) - war consists of a series of negotiations: if a round of negotiations fails, then the two sides fight a battle in which the victor 
takes a "fort" from the opposing side, the two parties update their beliefs, and negotiations resume; the cycle repeats itself until one side's supply of forts has been depleted, or their beliefs have sufficiently converged that they are able to reach a negotiated agreement. Their approach is innovative, particularly because it relaxes the common priors assumption, permitting belligerents to have heterogeneous beliefs even when exposed to the same information (for a critique, see Fey and Ramsay, 2006).

However, resolve in their game is operationalized as the supply of forts, one of which changes hands at the end of each battle. In this respect, there is nothing in their model that sets resolve apart from any other resource exchanged or depleted in war - as the authors acknowledge in their assessment that forts in modern war represent "units of resolve or strategically important pieces of land", but could be "any particular form of military resource that is necessary for the two nations to continue to fight and that can trade hands." (p. 788-9). Indeed, the "fort" metaphor is derived from an earlier work that never directly mentions resolve, referring to forts instead as "a discrete approximation of what nations are fighting over" (Smith, 1998 , 303). Since resolve functions in their approach as a generic type of resource, it is unclear what the model gains from labeling the resource "resolve" rather than cannonballs or horses, both of which are also subtractable resources that can change hands, but which actors need in order to continue to fight. ${ }^{10}$ After all, the strength of formal theoretical approaches is the extent to which they force scholars to clearly define their concepts and specify the assumptions needed for a theory's predictions to hold (Martin, 1999). Even outside the realm of rational choice, if resolve is simply doing the work that capabilities or intentions can do, then our theories of world politics are less parsimonious than they otherwise could be. Instead of portraying states as grappling with uncertainty over others' capabilities, intentions, and resolve, we could simply substitute the last property with the first two. To answer

\footnotetext{
${ }^{10}$ In this sense, resolve in their model functions as what Alfred Hitchcock called a "MacGuffin": the object that motivates all of the action, but whose intrinsic properties are irrelevant (the microfilms in North by Northwest, the statue in the Maltese Falcon, the briefcase in Pulp Fiction, and so on). See Dolar (1992).
} 
this question, we need to step back and adopt a broader view, contextualizing resolve's portrayal in IR in light of how the phenomenon is understood elsewhere in the social sciences.

\subsection{Two stories about resolve}

Figure 1.2: A spectrum of theories about resolve

\begin{tabular}{|c|c|c|}
\hline Dispositional & Interactionist & Situational \\
\hline Resolve as a trait or faculty & $\begin{array}{l}\text { Resolve as a function of both } \\
\text { traits and stakes }\end{array}$ & $\begin{array}{l}\text { Resolve as a costs / stakes of } \\
\text { potential outcomes }\end{array}$ \\
\hline Cognitivist: privileges agency & $\begin{array}{l}\text { Interactionist: both structure and } \\
\text { agency }\end{array}$ & $\begin{array}{l}\text { Behaviorist: privileges } \\
\text { structure }\end{array}$ \\
\hline $\begin{array}{l}\text { Capabilities and intentions } \\
\text { independent from resolve }\end{array}$ & $\begin{array}{l}\text { Capabilities and intentions at } \\
\text { least partially independent } \\
\text { from resolve }\end{array}$ & $\begin{array}{l}\text { Capabilities and intentions } \\
\text { interdependent with resolve }\end{array}$ \\
\hline $\begin{array}{l}\text { Internal commitment devices } \\
\text { (self-control) }\end{array}$ & $\begin{array}{l}\text { Internal \& external } \\
\text { commitment devices }\end{array}$ & $\begin{array}{l}\text { External commitment devices } \\
\text { (tying hands) }\end{array}$ \\
\hline $\begin{array}{l}\text { e.g. Schelling 1966, Rosen 1972, } \\
\text { Mueller 1980, Pape 1996, } \\
\text { Krauthammer } 2009\end{array}$ & & $\begin{array}{l}\text { e.g. Snyder and Diesing 1977, } \\
\text { Fearon 1994, Kydd 2003, } \\
\text { Sartori } 2005\end{array}$ \\
\hline
\end{tabular}

There are two types of stories social scientists have told about resolve: an inside story, and an outside one. ${ }^{11}$ The outside or situational story, popular in behaviorist branches of psychology and economics, understands resolve as the costs or stakes that an actor faces in a particular situation; the inside or dispositional story, more common in philosophy and social psychology, views resolve as something internal to the actor itself. We can array these two poles on a spectrum shown in Figure 1.2a, with an interactionist perspective in between. The poles are ideal types, and

\footnotetext{
${ }^{11}$ I borrow this framing device from Hollis and Smith (1990), but whereas their two sets of stories are epistemological - referring to how we know of or come to understand social phenomena mine are ontological, referring to assumptions about what the phenomena are.
} 
many IR scholars adopt positions that veer away from these two extremes, ${ }^{12}$ but as I show below, it is notable just how much of the IR literature can be characterized as residing at one of the two ends of the spectrum. In the section that follows, I briefly introduce each perspective and show how they differ from one another, both in terms of second-order questions of ontology, and first-order substantive questions in IR theory. One of the reasons why our understanding of resolve in IR is so muddled is because we have borrowed assumptions from various points across the spectrum, making it difficult to produce a coherent account of what resolve is and how it works.

\section{Situational THEORIES OF RESOLVE}

Starting at the right hand side of the spectrum, we can imagine a situational theory of resolve, which argues that actors will display resolve when the stakes are high, and will be irresolute when the stakes are low. Situational theories of resolve were briefly ascendant in economics and experimental psychology during the movement towards behaviorism: operant conditioning studies with pigeons (Herrnstein, 1970; Rachlin and Green, 1972) and early delayed gratification studies with young children (Mischel and Grusec, 1967) emphasize the importance of external reinforcement, observing the extent to which people and pigeons alike respond predictably to external stimuli. Behaviorists argue that self-control is primarily a function of two phenomena: the magnitude of the reinforcer, and the delay involved (Logue, 1988, 665); by manipulating the size of the reinforcer and the length of the delay, actors will display self-control problems, and reverse their choices (for a review, see Logue, 1988, 1998). In the behaviorist paradigm, self-control is therefore situational rather than dispositional: the higher the level of abstraction a reward is, the greater we discount it (Gifford, 2002; Trope and Liberman, 2003),

\footnotetext{
${ }^{12}$ For example, Powell (1987) and Morgan (1994) treat resolve as a function of both situational features (e.g. cost-benefit calculations about the stakes) and dispositional ones (risk orientation), although they do not incorporate them into the kind of thicker interactive framework I describe in Chapter 2.
} 
and what philosophers refer to as "weakness of will" (Stroud and Tappolet, 2003; Elster, 2006; Hoffmann, 2008) is a consequence not of a lack of character (Green and Fisher, 1988, 684), but to intersecting hyperbolic discounting curves (Ainslie, 1992, 2001; Frederick, 2006).

Hyperbolic discounting has yet to make much of an impact on IR (although see Streich and Levy, 2007; Krebs and Rapport, 2012), but situational conceptualizations of resolve should nonetheless be easy to imagine for a political science audience, particularly amongst rational choice scholars. Much of the IR literature has defined resolve in terms of costs and benefits, especially in the crisis bargaining literature: an actor will be resolved in a crisis bargaining situation when the stakes are high relative to the costs, and irresolute when they are low, such that actors are systematically more resolute about some issues than about others (Snyder and Diesing, 1977; Betts, 1980; George and Simons, 1994; Powell, 2003; Trager, 2011). As can be expected, there is some conceptual ambiguity here: as Pape (1996) notes, some approaches fully reduce the "balance of resolve" to the "balance of interests" (Lebow, 1998; Arreguín-Toft, 2001) and thereby treat an actor's resolve as its cost of conflict (Snyder and Diesing, 1977; Gartzke, Li, and Boehmer, 2001; Kydd, 2003; Rauchhaus, 2006; Fey and Ramsay, 2011; Lake, 2010/11), while others treat an actor's costs as one of several determinants of resolve (Morrow, 1985, 1987; Mattes and Morgan, 2004), and others model an actor's cost of war and level of resolve as distinct types of private information altogether (Goemans, 2000). These distinctions are not just semantic, especially for deterrence theorists (Jervis, 1979): why bother maintaining a general reputation for resolve if resolve is merely a byproduct of the particular interests at stake in a situation?

Situational theories of resolve in IR raise an additional theoretical issue: how do actors weigh the different types of costs at stake? IR scholars have focused heavily on military fatalities as the "human cost" of war (Mueller, 1971; Gartner and Segura, 1998; Gelpi, Feaver, and Reifler, 2005 / 06; Gelpi, Reifler, and Feaver, 2007; Gartner, 2008a), but war also has economic costs (Caverley, 2009/10; Geys, 2010), 
and is also rife with opportunity costs. After all, although fighting imposes a considerable burden, so too does defeat (Filson and Werner, 2007b) and withdrawal (Sullivan, 2008), which may bear reputational costs that will long outlast the immediate physical consequences of the conflict. In this sense, one can imagine a wide range of situational theories of resolve based upon the particular types of costs that matter, whether reputational, economic, or otherwise. What all of the theories at this portion of the spectrum share, though, is the automaticity through which these costs translate into action: sufficiently raise the costs, and all actors will back down (Pape, 1996; Sullivan, 2007), irrespective of political ideology, regime type, or any other internal factor inside the actor that might affect how they respond to these external stimuli.

\section{DisPoSitionAL THEORIES OF RESOLVE}

Situational theories of resolve carry many advantages: they are parsimonious, base their predictions on observable and manipulable factors, and correspond with much of what ordinary intuition tells us about how actors behave when the stakes are high. Ultimately, though, if we think of resolve as maintaining a policy despite contrary inclinations or temptations to back down, it implies that resolve involves resisting situationally-induced pressures to retreat or reverse course. When we talk about someone being resolved, we are usually referring to something that emanates from within rather than merely dictated from without, a property of the actor itself rather than a feature of the situation. Foch and Napoleon contrasted resolve with material factors out of the assumption that resolve involves being unmoved by material disadvantages, and in the historical cases we associate with resolve for example, the French persisting at the Battle of Verdun despite suffering nearly a third of a million dead, injured, or missing, the Guomindang's long and grueling fight against the Japanese during the second Sino-Japanese War, the Paraguayans pressing on in the devastating War of the Triple Alliance, and so on - we know that the French, Chinese, and Paraguayans were resolved not because they acted 
in accordance with the costs inherent in the situation, but because they persisted despite these costs. Our lay theories about resolve, then, are dispositional in nature.

This dispositional intuition is bolstered both by much of the literature on willpower and self-control throughout the social sciences, as well as by work in IR itself. Sociologists and psychologists have tended to treat self-control or willpower as an "individual difference" (Anastasi, 1937) similar to a personality trait (Gottfredson and Hirschi, 1990; Caspi, 2000), and like other such traits, possibly genetically inherited (Beaver et al., 2010; Boutwell and Beaver, 2010). The ego-depletion model of willpower recently popular in psychology (Baumeister, Vohs, and Tice, 2007; Galliot et al., 2007; Hagger et al., 2010) treats self-control as a trait activated in multiple spheres: attention control, emotion inhibition, thought suppression, volition and choice, and so on. Regardless of whether the specific task at hand requires stifling emotional reactions, maintaining handgrip stamina, or eating radishes while facing a plate of freshly-baked cookies, the findings suggest that the same psychological processes are at work, and offer considerable evidence in favor of folk intuitions about willpower as a trait better developed in some individuals than others, which is depleted over time, strengthened through training, and so on.

Although these psychological findings are all at the individual level, IR scholars have made similarly dispositional arguments about resolve at the collective level. Sartori $(2005,45)$, for example, notes that although some deterrence theorists have viewed resolve solely as a function of the issues at stake in a particular crisis, others "maintain that it is an enduring, dispositional quality, that some states generally are more willing to fight than others." Similarly, both formal theorists and empirical IR scholars have noted that actors vary in their sensitivity to costs: Mueller (1980) suggests that one of the American strategic failures in the Vietnam War was assumption that sufficient costs would push the North Vietnamese past their "breaking point"; Pape (1996) similarly notes that states vary in their vulnerability to coercion, Jervis $(1976,51)$ refers to states varying in their "willingness to pay," while 
Rosen (1972) argues that states vary in their "willingness to suffer."13 Two of the dominant research traditions in the democracies in war literature - the "selection effects" work that predicts that democracies are more likely to win their wars because they are more cautious in choosing which wars are worth fighting (Siverson, 1995; Reiter and Stam, 1998b; Valentino, Huth, and Croco, 2010), and the literature investigating whether the advantages of democracy in battle decline over time because democratic publics are too casualty-shy to sustain lengthy combat operations (Bennett and Stam, 1998; Gartzke, 2001; Reiter and Stam, 2002; Sullivan, 2007, 2008; Gelpi, Feaver, and Reifler, 2009; Lyall, 2010) — both acknowledge the importance not just of costs, but of cost sensitivity. In much of the literature, the issue is not whether democracies have higher costs of war than autocracies (although see Schultz 1999), but how tolerant they are of the costs. ${ }^{14}$ In the language of economics, some states have more elastic demand curves for war than others: cost-sensitive actors will lose their appetite for conflict if the price is high, while other actors have relatively inelastic demand curves, and will continue to support the conflict despite the raised costs. A recent wave of game theoretic work in IR has explicitly incorporated cost sensitivity into their models (Stam, 1996; Slantchev, 2003; Filson and Werner, 2004; Mattes and Morgan, 2004; Filson and Werner, 2007b), which, even if relying on overly simplistic assumptions about the relationship between regime

\footnotetext{
${ }^{13}$ Stam (1996) suggests this variation occurs crosstemporally as well: France lost hundreds of thousands of troops at the Battle of Verdun yet remained in the First World War, but surrendered in the Second World War after having lost 50,000 soldiers over the course of five weeks (p. 19).

${ }^{14}$ This literature varies as to where democratic cost-sensitivity comes from: some accounts point to a free press and open information environment (Johnson et al., 2006), others emphasize institutions promoting foreign policy accountability (Koch and Gartner, 2005), and others suggest that cost-sensitivity stems from political ideology, with left-leaning political parties being more sensitive to the costs of war than right-leaning ones (Palmer, London, and Regan, 2004; Koch and Sullivan, 2010). It may seem counterintuitive to characterize institutional explanations as being dispositional - particularly from the perspective of comparative politics, which characterizes domestic political institutions as contextual factors - but in the context of international politics, "inside", dispositional accounts are those that refer to characteristics or attributes of states, while "outside", situational accounts involve the environment or context in which the state is operating. Thus, what Waltz (1959) called "second-image" accounts are dispositional rather than situational; innenpolitik is very much an inside story rather than an outside one. I return to this point in the discussion of the large- $\mathrm{N}$ analyses in Chapter 6. The question of where we locate agency in collective actors like states is an interesting one (Wendt, 2004; Wight, 2004), but outside the scope of this project.
} 
type and cost sensitivity (Maoz and Siverson, 2008), is nonetheless useful for our purposes, since cost sensitivity is portrayed in a manner that makes it inversely related to resolve (see also Iyengar and Monten 2008). Thus like situational theories of resolve, dispositional theories of resolve are well represented in both IR theory and the social sciences more broadly.

\section{COMPARING THE TWO STORIES}

As discussed above, dispositional and situational theories of resolve differ in the extent to which they conceptualize resolve as an internal or an external phenomenon. Dispositional theories of resolve treat it as an internal trait - part of the actor itself - whereas situational theories of resolve treat it as an external phenomenon - a feature of the environment in which the actor is operating. The two sets of theories therefore offer strikingly different implications both in terms of second-order questions of ontology, and first-order questions in IR theory.

\section{The agent-structure problem}

For example, they offer divergent approaches to the agent-structure problem (Wendt, 1987; Dessler, 1989; Carlsnaes, 1992; Jabri and Chan, 1996; Doty, 1997; Bieler and Morton, 2001). Whereas situational theories of resolve treat the concept as a structural phenomenon, dispositional theories of resolve privilege agency instead. Perhaps reflecting the language of levels of analysis (Singer, 1961; Onuf, 1995), the relationship between agents and structures has typically been portrayed in IR through the use of vertical metaphors in which agents are understood to be embedded in, but nonetheless, "below" structures that constrain and enable agents' choices, such that Wendt (1999) writes about "bottom-up" versus "top-down constitution." This external vision of structure is the one adopted by situational theories of resolve, in which actors are impeded or encouraged to pursue particular goals by the costs or benefits imposed by the strategic environment. Structures, though, are not just external to the agent, and we can imagine agents constrained or enabled both ex- 
ternally and internally (Todd and Gigerenzer, 2003), in that agents also have interior organizational structures (Wendt, 1987, 359), internal dynamics of agency that mirror what psychologists call self-regulation - not just regulation of the self, but regulation of certain parts of the self by other parts of the self (Baumeister and Vohs, 2004). Dispositional theories of resolve focus on these internal moving parts: as Kuhl (1988) points out, we call it "self-control" out of the assumption that the concept refers to a feature or faculty independent from the environment. ${ }^{15}$

\section{Commitment problems}

It is for this reason that advocates of dispositional and situational theories of resolve also have such opposing views of commitment problems, instances where actors are unable to maintain behavioral consistency over time (Becker, 1961). The same rationalist IR theorists who define resolve situationally in terms of the costs an actor faces also perceive commitment problems through a situational lens: actors will experience commitment problems when a plan or agreement that was in their interests $e x$ ante is no longer in their interests ex post due to changes in the environment in which an actor finds itself (Kydland and Prescott, 1977; Powell, 2006; Beardsley, 2008). Thus, for situationalists, commitment problems are resolved through the use of external commitment devices - "tying hands", as in Fearon (1997) that render behavioral consistency incentive-compatible (e.g. Walter 1997). ${ }^{16}$ The dispositional accounts of resolve popular in psychology and parts of economics, on the other hand, lead to an understanding of commitment problems from an internal perspective, in which commitment problems involve a failure to resist temptation and engage in self-regulation, but can be rectified by internal commitment devices

\footnotetext{
${ }^{15}$ Philosophers from Plato onwards called this feature the "will", a volitional faculty of the mind that translates thought into action (Arendt, 1971; Davenport, 2007). In this sense, resolve is central to many of the aspects we associate with agency: the sense of action being guided autonomously (Ryan and Deci, 2006), the ability "to have acted otherwise." (Doty, 1997, 372), and the capacity for second-order volitions (what Frankfurt $(1971,15)$ called the freedom for an individual "to want what he wants to want").

${ }^{16}$ This is also reflected in language suggesting that actors "create" resolve (Morgan, 1990, 283284) through commitment devices; if resolve truly was purely dispositional, it would not need to be created in the first place.
} 
— the exercise of willpower or self-control (Gifford, 2002; Bénabou and Tirole, 2004; Benhabib and Bisin, 2005; Hofmann, Friese, and Strack, 2009).

\section{Capabilities, intentions, and resolve, redux}

Similarly, the two bodies of theories espouse very different predictions about the relationship between (i) resolve and capabilities, and (ii) resolve and intentions. Dispositional theories of resolve see all three concepts as distinct: like in force activation models (March, 1966), actors can have sophisticated capabilities but lack the will to fully apply them, while weakness of will means that actors can intend to do something - commit to a war, push the other side to the brink in a crisis bargaining scenario - but lack the resolve to follow through. As Sheeran (2002) notes, there is often a marked gap between our intentions, and the actions in which we actually engage, one of the many "canyonesque" gaps that characterize human behavior (Epley and Caruso, 2008, 297). The three concepts may not be entirely independent of one another in dispositional accounts, but the automaticity found in the outside story is not present in the inside one.

Situational theories, on the contrary, see the concepts as highly interrelated, causally if not definitionally. First, as actors' relative capabilities increase, their cost of fighting will decrease, effectively rendering them more resolved (Snyder and Diesing, 1977; Morrow, 1985), such that increases in relative capabilities produce increased resolve. Second, if actors are self-interested utility maximizers, then inasmuch as we can overcome the problem of other minds and study motivations at all (Morgenthau, 1985, 5), we can derive actors' intentions from the costs and benefits of particular policies: if the cost of a policy increases, actors will be less interested in pursuing it, and will act accordingly. For the outside story, then, cost, capabilities, intentions, and resolve all become highly intertwined. 


\subsection{Conclusion}

It was argued here that although resolve is one of the most central independent variables in IR theory, the term is often used in imprecise and incompatible ways. Yet the cost of this neglect goes beyond lexical inconsistency: resolve has been turned into a catch-all residual category used to explain everything that our traditional theories cannot (March, 1966, 61; Baldwin, 1979; Jervis, 1979, 316; Rummel, 1975b, 275; Ray and Vural, 1986), threatening to reduce the concept to a set of "posthoc explanations for otherwise perplexing conflict outcomes"' (Sullivan, 2007, 497). Integrating the IR literature on resolve with its counterparts in other fields, I argued that there have traditionally been two types of stories that social scientists have told about resolve: an inside, dispositional account in which resolve is treated as a trait, and an outside, situational account in which resolve is understood as the costs and stakes an actor faces.

The two stories differ not just in terms of metatheoretical assumptions, but in their policy implications. The neoconservative rhetoric of resolve evident in Krauthammer's declaration that "decline is a choice" understands resolve dispositionally, such that liberals who show a lack of resolve are displaying a kind of weakness of character to be rectified by self-discipline. Indeed, just as a lack of resolve is typically understood as a character flaw in the domestic realm (Baumeister and Exline, 1999), a lack of political will is typically understood as a sin in the political arena, responsible for genocides, defeats, and disappointments (Power, 2002). The liberal pundit Matthew Yglesias (2006) suggests that contemporary American conservative foreign policy discourse is marred by a "Green Lantern theory of geopolitics," where all military obstacles can be overcome with sufficient willpower, and the journalist Jonathan Chait (2011), writing in The New Republic, argued that "in the neoconservative world, mighty declarations of willpower always trump puny arithmetic." It is not coincidental that Senator Joe Lieberman's retrospective on the tenth anniversary of the September 11th attacks published in Foreign Policy was entitled "A Decade of Resolve." (Lieberman, 2011) If, on the other hand, resolve 
is purely situational, then it is less like a switch that can be flipped by "digging deep," and is instead more closely tied to the stakes, costs and benefits that actors find themselves facing. Decline is no longer a choice, but the product of broader forces beyond any one actor's control. As is the case with any structural theory, situational views of resolve leave less room for agency: actors may try to transform their cost-benefit calculus by engaging in various commitment devices to change the stakes, but these may come with other political costs attached.

Situational and dispositional theories of resolve therefore not only come to very different understandings of what resolve is, but also different beliefs about how it functions. Although each account offers some insight into how resolve operates, neither offers a satisfactory explanation by itself. If we intuitively believe that some actors are more resolute than others in general, but also acknowledge that an actor's level of resolve will vary based on the situation it finds itself in, then resolve seems to have both dispositional and situational components. The challenge I take up in this dissertation is how these two sets of components interact, linking specific dispositional traits to situational features to explain why certain costs of war loom larger in the decision-making calculus of certain types of actors. 


\section{Chapter 2: An Interactionist Theory of Resolve}

Although thinking about resolve in terms of inside and outside stories is compelling in that each approach offers some insight into how resolve operates, this dichotomy poses a significant problem in that neither account offers a full or otherwise satisfactory account of the phenomenon under investigation. If we intuitively believe that some actors are more resolute than others in general, but we also assume that an actor's level of resolve will depend on the situation it finds itself in, then resolve seems to have both dispositional and situational components; given the choice between an inside story and an outside one, the most attractive option would seem to be to choose both. And yet, since the two accounts are defined in opposition to one another, both ontologically (that is, they disagree on what resolve is) and substantively (that is, they present divergent predictions on how it operates), one cannot situate oneself at both positions simultaneously: if both accounts are correct, than neither one is. The solution is to adopt an interactionist approach that splits the spectrum down the middle, a via media that views resolve as a state shaped by both dispositional and situational factors.

This chapter begins with a brief introduction to interactionist theories, elaborating what scholars of world politics gain by thinking of resolve in an interactionist manner. I then use this framework to introduce two situational variables (the human costs of fighting, and the costs of backing down) and three dispositional variables (time and risk preferences, and cultures of honor) to create an interactionist theory of resolve, presenting a series of hypotheses elaborating why certain types of actors are more or less sensitive to certain types of costs of war. 


\subsection{Three motivations for interactionism}

Before presenting an interactionist theory of resolve, it is worth noting what such a theory entails, particularly because interactionism is a term with multiple meanings, and the type of interactionism I discuss here differs somewhat from some of its homonyms (for example, symbolic interactionism in sociology). By interactionism I mean a type of theory that emphasizes the interplay between actors and their environment, and thus explains phenomena as a function of both unit-level attributes and situational constraints. Although interactionism is by no means foreign to political science - as evident in classic pieces in the study of foreign policy decision-making such as Harold and Margaret Sprout's (1957) work on "manmilieu interactions" - contemporary political science's emphasis on parsimonious theories with fewer moving parts (e.g. Waltz, 1979) has meant that the field is less self-consciously interactionist than disciplines like psychology, where interactionism emerged as a way of reconciling personality psychology's emphasis on traits and individual differences with social psychology's emphasis on the power of the situation in which individuals are immersed (Endler and Magnusson, 1976; Snyder and Cantor, 1998; Mischel, 2004; Reynolds et al., 2010).

As summarized in Table 2.1, interactionist theories in the social sciences tend to be employed for three different types of motivations, each of which relies on a different set of assumptions about the causal mechanisms driving the phenomenon under investigation. First, social scientists are occasionally simply interested in partitioning variance between dispositional and situational variables: incorporating both types of factors into a model, and determining the relative contribution of each one. This approach was popular during the height of the person-situation debate (Sarason, Smith, and Diener, 1975), as psychologists attempted to determine just how important each type of explanation was in explaining behavior. Nonetheless, it represents the thinnest type of interactionism discussed here, since it is only interactionist in that it includes both situational and dispositional variables in an additive model, without necessarily featuring the more elaborate interplay 
Table 2.1: Rationales for interactionist theorizing, from thinnest to thickest

\begin{tabular}{|c|c|c|}
\hline Motivation & Causal pathway & Research question \\
\hline $\begin{array}{l}\text { 1. Partitioning } \\
\text { variance }\end{array}$ & Main effects & $\begin{array}{l}\text { How much variation in a phenomenon is due to } \\
\text { situational factors rather than dispositional ones? }\end{array}$ \\
\hline $\begin{array}{l}\text { 2. Interactive } \\
\text { theories }\end{array}$ & Interaction effects & $\begin{array}{l}\text { Multiplier effects: do certain situational factors } \\
\text { enhance the impact of dispositional ones, or vice- } \\
\text { versa? }\end{array}$ \\
\hline & & $\begin{array}{l}\text { Scope conditions: under what disposition-situation } \\
\text { combinations is a phenomenon more likely to } \\
\text { occur? }\end{array}$ \\
\hline $\begin{array}{l}\text { 3. Definition of } \\
\text { the situation }\end{array}$ & $\begin{array}{l}\text { Direct and } \\
\text { indirect effects }\end{array}$ & $\begin{array}{l}\text { How do dispositional factors affect how actors } \\
\text { perceive their environment? }\end{array}$ \\
\hline
\end{tabular}

between the two sets of variables found in the other types of interactionism discussed below.

The second type of interactionist theorizing, which I call interactive theories, are what most social scientists tend to think of when they think of interactionism. In an interactive theory, the effect of one set of variables upon the dependent variable of interest is conditional upon (or is "moderated by", or "interacts with") another set of variables (Jaccard and Turrisi, 2003), expressed econometrically in the form:

$$
Y=B_{0}+B_{1} X+B_{2} Z+B_{3} X Z+\epsilon, B_{3} \neq 0
$$

where $X$ is the focal predictor, $Z$ the moderator, and $X Z$ the interaction term. As the above definition makes clear, interactionist theories and interactive theories are only partially overlapping categories, since not all interactionist theories are interactive (Schmitt, 2009), and not all interactive theories are interactionist. After all, as in the partitioning variance case discussed above, interactionist theories can specify additive rather than interactive functional forms, in which both dispositional and situational variables affect behavior, but through independent pathways (the case where $B_{3}=0$ ). For example, neoclassical realist theories are interactionist, since they look at how both domestic politics $(X)$ and the international system $(Z)$ affect state behavior (Rose, 1998; Schweller, 2003), but are not necessarily interactive, in that they do not all specify that the impact of one level of analysis is conditional 
upon the other (the $B_{3} \neq 0$ restriction). Conversely, not all interactive theories are interactionist: Zaller's (1992) model of political behavior posits that an individual's level of knowledge $(X)$ interacts with her partisan identification $(Z)$ in affecting her political behavior, but since $X$ and $Z$ are both unit-level attributes, the theory is interactive but not interactionist, properly speaking. Nonetheless, many theories in political science are both interactionist and interactive in that they specify that the impact of a set of variables at one level of analysis depends on the values of a set of variables at a different level (e.g. Walt, 1985; Grieco, 1993; Herrmann and Fischerkeller, 1995). ${ }^{1}$ As such, interactive interactionist models are especially desirable when seeking to model multiplier effects (e.g., when certain dispositional and situational factors are both present, an actor will be even more resolute), or aiming to incorporate scope or boundary conditions (e.g. the idea of "trait activation" (Tett and Gutterman, 2000), which examines the conditions under which certain traits are more likely to be expressed) into the theory.

A third type of interactionist theorizing occurs when a social scientist is interested in studying the impact of what the early twentieth-century sociologist W.I. Thomas called the "definition of the situation." Whereas the previous two approaches understood dispositional and situational factors as entirely separate independent variables, this third form of interactionist theorizing questions the existence of an objective external environment apart from the observer, and notes that actors' dispositions affect their perception of the context they find themselves in. As such, although there are some cases where situations impact behavior through direct pathways - Wolfers' (1962) example of a fire in a crowded theatre - there are others where situations impact behavior through indirect (dispositional) pathways instead, and thus this third type of interactionist theorizing bears some similarities to meditation analysis, with its emphasis on direct and indirect effects (Baron and Kenny, 1986; Imai et al., 2011). Not surprisingly, definition of the situation theo-

\footnotetext{
${ }^{1}$ I temporarily set aside the question of whether some of these theories are better expressed by a Boolean formulation rather than an interactive one (Braumoeller, 2003; Braumoeller and Carson, 2011), exploring this possibility in Chapter 5.
} 
rizing is not only explicit in much of the cognitive revolution in psychology (Herrmann, 1988), but is also implicit in constructivism in IR theory as well (Wendt, 1999), which argues that anarchy (the situation in which states find themselves) is what states (and their dispositions) make of it (Wendt, 1992). In the empirical tests in Chapters 3 and 4 I employ some mediation analyses, but for the most part stick to moderation analyses, probing the extent to which situational effects are conditional upon dispositional characteristics.

Finally, it is important to note that from a philosophy of science perspective, these three sets of rationales for interactionist theorizing discussed above render interactionism desirable regardless of the "academic sect" (Lake, 2011) to which one pledges allegiance. For scientific realists, an interactionist via media is obviously attractive if we understand resolve as shaped by both situational factors and dispositional ones, since the essence of ontological realism is the premise that our theories should correspond to the phenomena about which we're theorizing (Joseph and Wight, 2010). Yet even among positivists or methodological instrumentalists, however - who view assumptions as neither true nor false, but only more or less useful (e.g. Friedman, 1953), and thus might be tempted for sake of parsimony to restrict their analyses to just one level of analysis - an interactionist approach is nonetheless advantageous, since modeling only one half of an interactive relationship produces omitted variable bias, diminishing our theories' predictive utility - and as we shall see in Chapter 5, interactive models of resolve display higher predictive power than their additive counterparts.

One obstacle to thinking about resolve in an interactionist fashion is a metaphysical one - how can resolve be both dispositional and situational? This challenge is particularly vexing from a dispositional perspective, since the very idea of a "heroic will" (Smith, 2001; Davenport, 2007) implies a self-motivated force independent of the environment, which raises questions about construct validity (namely, whether the phenomenon being studied in an interactionist account of resolve is in fact resolve at all). The solution I employ, rather than viewing resolve 
as both a disposition and a situation, is to understand resolve as a state that has both dispositional and situational causes, just as psychologists coming out of the person-situation debate have come to view "mentalistic" (Dennett, 1987) concepts like emotions and personality traits as a series of successive states that are affected by both dispositional and situational triggers. In Appendix A.1, I offer a further discussion of this point, using the person-situation debate in psychology to make the above discussion more concrete, revisiting it again at the end of Chapter 6.

\subsection{An interactionist theory of resolve in military interventions}

Although resolve obviously manifests itself in any number of domains (from crisis bargaining to international trade negotiations to counterterrorism campaigns), I focus specifically on resolve in the realm of military interventions, a move that is advantageous for three reasons. First, although much of the social science literature on willpower treats resolve as cross-situational rather than domain-specific (e.g. Baumeister, Vohs, and Tice, 2007), this is less likely to be the case if, as an interactionist theory expects, resolve has strong situational determinants: an actor may display strong political will in one issue, but very little on the next, and the determinants of resolve in each case may be different. By restricting the study of resolve to a single domain, we therefore gain greater leverage over the causal mechanisms that underlie it in this specific area.

Second, if resolve is conceptualized as something akin to persistence - a steadfastness of purpose that maintains a policy over time despite contrary inclinations or temptations to back down - international interventions are a perfect illustration of resolve at work, as leaders and their publics frequently intervene only to seek an exit option as the pressure mounts (Sullivan, 2008; Polsky, 2010; Koch and Sullivan, 2010). Third, military interventions represent one of the defining issues of the past 10 years, with questions about the sustainability of the U.S. missions in Afghanistan and Iraq occupying a prominent position in most Western countries'

foreign policy agendas. Understanding the factors that make some intervening 
states stay the course, and others cut their losses, is therefore of both theoretical interest and practical importance.

As a phenomenon driven by both situational and dispositional factors, resolve is ultimately likely to have a large number of causes. Our task as as political scientists, however, is not simply to amalgamate all of the factors that could plausibly affect actors' resolve and insert them into a "garbage can" model (Achen, 2002), but rather, to focus on the workings of small number of variables selected on theoretical grounds. ${ }^{2}$ The interactionist theory of resolve I propose below is thus not intended to capture the universe of potential causes of resolve, but rather, seeks to integrate research on resolve in IR with a parallel body of work elsewhere in the social sciences, opening up the black box of "costliness" by identifying two situational features - the cost of fighting, and the costs of backing down - and linking them with four dispositional characteristics - time preferences, risk preferences, cultures of honor, and trait self-control - interactions between which can explain why certain types of actors are more or less sensitive to certain types of costs of war.

SitUATIONAL DETERMINANTS OF RESOLVE: OPENING UP THE BLACK BOX OF "Costhiness"

Situational theories of resolve, like all rationalist theories of war, argue that leaders are motivated by a logic of consequences (March and Olsen, 1998) and therefore seek to make choices that maximize their utility, weighing the perceived costs and benefits of options when deciding which ones to enact. According to this argument, actors confronted with low costs of war will be more resolved than those with high costs of war; states with high stakes on the line will be more resolute than those with low stakes. As is the case with many structural theories, however, a purely situational view of resolve is indeterminate, since cost-benefit calculations are not

\footnotetext{
${ }^{2}$ As Achen (1977) argues, the purpose of quantitative social science is not merely to maximize an $R^{2}$ statistic. For a similar argument, see King (1991). I return to this point in Chapter 6.
} 
always obvious. Although the IR literature has focused considerable attention on how uncertainty (Fearon, 1995; Powell, 2004) and overconfidence (Blainey, 1973; Johnson, Wrangham, and Rosen, 2002; Fey and Ramsay, 2007) lead rational actors to pursue policies that are nonetheless inefficient ex post, less attention has been directed towards a different complication: how do actors decide which costs of war count as costly?

Classical models of utility theory like those proposed by von Neumann and Morgenstern (1944) rely on unidimensional utility functions based on a single metric; actors can be assumed to have complete preferences because utility acts as a common denominator that enables direct comparisons of the value derived from any pair of alternatives (Etzioni, 1986; Brennan, 1989). Particularly in IR contexts where decision-makers weigh options using multiple criteria, however, it is often analytically useful to rely instead on multiattribute utility functions (Dorfleitner and Krapp, 2007). As Simon (1985) argued, our theories of rational utility maximizers are only as useful as our auxiliary assumptions about where actors derive their utility from; although situational theories of resolve appear parsimonious and clear in their predictions, some of this clarity comes from a sort of "iceberg parsimony," where much of the theory's mass is hidden under water. ${ }^{3}$ It is one thing to say that actors will be resolved when their payoff structures tell them to be (Fearon, 1995), but it is another thing to specify where these payoffs come from in the first place.

After all, the costs of war come in many forms - economic costs (Geys, 2010), human costs in the form of casualties (Mueller, 1971; Gelpi, Feaver, and Reifler, 2009), reputational costs, personal political fortune (Bueno de Mesquita and Siverson, 1995), opportunity costs (Nincic, 1997), sunk costs (Boettcher and Cobb, 2009), and so on - but leaders have to come to some sort of decision about which costs outweigh others. For example, accounts of war in political economy suggest that states make conscious decisions about sheltering capital or labor when fighting (Gartzke, 2001; Caverley, 2009/10), implying that some states will be more sen-

\footnotetext{
${ }^{3}$ This charge is similar to the constructivist critique of structural realism for basing its explanatory power on implicit assumptions about the "distribution of interests" (Wendt, 1999, ch. 3).
} 
sitive to the human costs of war, while others will be more concerned about economic costs. It is frequently argued that the United States has been so enamored with the capital-intensive warfare favored by the Revolution in Military Affairs (RMA) because of concerns that the American public is willing to spend money, but not lives (Smith, 2005). Similarly, a number of scholars of international conflict have argued that states deciding whether to continue to prosecute a war must pay attention both to the cost of continuing to fight, and the costs of terminating the war (Filson and Werner, 2007a; Sullivan, 2007, 2008), the latter of which involves a sunk cost argument (Arkes and Blumer, 1985), concerns about how withdrawal will affect reputation for resolve (Mercer, 1996), and an acknowledgment of the greater concessions that must be made at the bargaining table (Koch and Sullivan, 2010). The question, then, is not just about how attentive leaders are to costs - the notion of cost-sensitivity - but how leaders weigh different kinds of costs, an aggregation process sufficiently complex that Schultz $(2001 \mathrm{a}, 34)$ views it as a particularly "vexing" source of asymmetric uncertainty. ${ }^{4}$

As illustrated by the left-hand panel in Figure 2.1, I focus on two different types of costs of war as situational determinants of resolve in the context of military interventions: the costs of fighting - measured chiefly by casualties - and the costs of backing down, which include both the intrinsic stakes of the mission, and the reputational costs of withdrawal.

\section{The costs of fighting: casualties}

Once a war or military intervention has begun, the first situational cost likely to affect an actor's resolve is casualties, ${ }^{5}$ frequently argued to be the dominant indicator decision-makers use to judge a war's progress (Gartner, 1997, 2008a), as well

\footnotetext{
${ }^{4}$ The judgment and decision-making literature has focused on this subject in considerable detail; work on mental accounting, for example (Thaler, 1985) focuses on the role that mental categorizations play in coding joint or multiple outcomes.

${ }^{5}$ Although "casualties" is often used in the military community to refer both to the number of dead and the number of wounded, I follow the rest of the casualty sensitivity literature in using casualties to refer to deaths alone.
} 
Figure 2.1: Resolve as a dependent variable

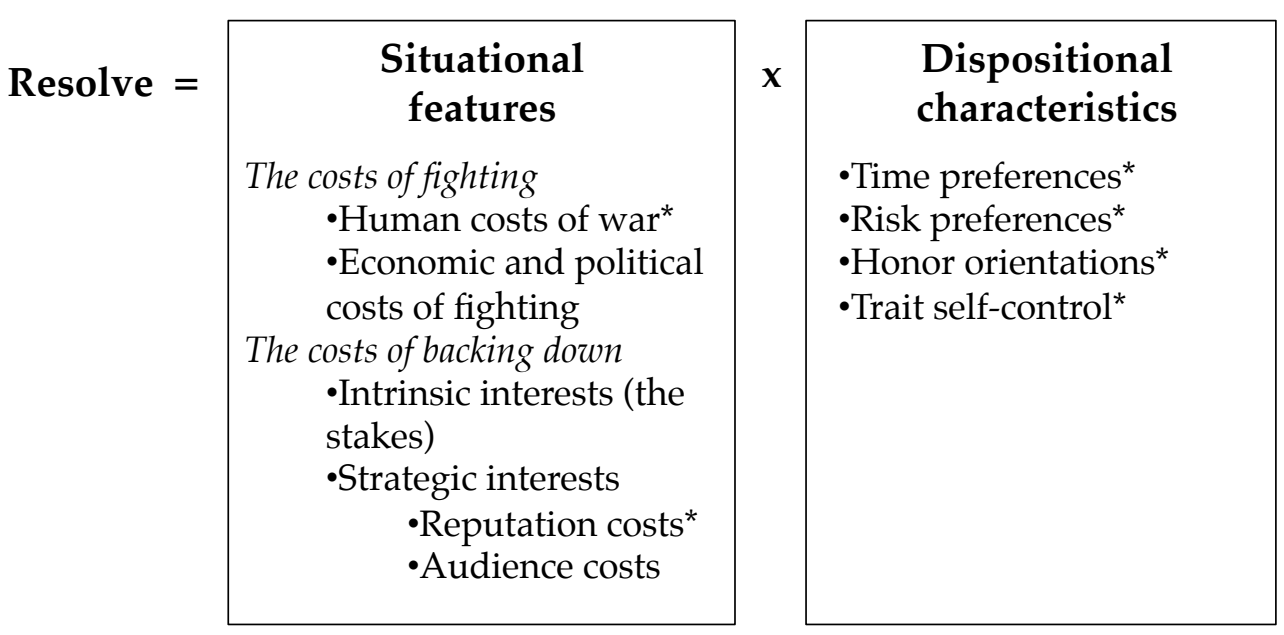

*Asterisks denote the variables studied in the lab and survey experiments

as one of the major factors in the public's decision-making calculus when determining support for continued use of force (Mueller, 1971; Larson, 2000; Klarevas, 2002; Smith, 2005; Gelpi, Feaver, and Reifler, 2009; Myers and Hayes, 2010). Although casualties are not the only cost of fighting - both economic and political costs come to mind - they typically loom the largest in the public's consciousness. To be sure, the economic costs of war are often enormous (e.g. Bilmes and Stiglitz, 2012), but the human costs figure more prominently in how we think about war, presumably because human life is understood as sacred in a way that money is not (Tetlock, 2003). Although economists are comfortable calculating the value of a human life (Miller, 2000; Viscusi and Aldy, 2003), the general public is far less sanguine, and survey results suggest the public is extremely uncomfortable providing acceptable casualty levels for military scenarios (Boettcher and Cobb, 2006). Similarly, although war has political costs, these are often endogeneous to casualties, since casualties increase the salience of military operations, which are then more likely to engender political opposition. I therefore expect that the greater the human costs of war, the less resolve actors will display, leading to my first hypothesis:

H1: The higher the number of casualties, the less resolve actors will 
display.

\section{The costs of backing down: the issues at stake, and reputation}

The second type of cost likely to affect an actor's resolve are the costs an actor incurs by backing down, either during a conflict or in a crisis situation (Filson and Werner, 2007a). An actor's commitment to a policy is in large part dependent upon how satisfying the alternatives are (Agnew et al., 2007; Hoffman et al., 2009), so an actor is more likely to remain resolute when terminating a conflict or backing down on a threat would produce relatively unsavory consequences. When an actor backs down or terminates a conflict, she pays two types of costs, which we can understand using Jervis's (1979) distinction between intrinsic and strategic (or extrinsic) interests. First, the actor backing down inevitably sacrifices the intrinsic interests the tangible objects or issues at stake in the conflict - since surrendering means accepting your opponents' demands. How much is at stake in a conflict depends on the nature of the issues under dispute: for example, humanitarian operations are less salient than operations that implicate core national security interests (Jentleson, 1992), especially those concerning threats to the territorial homeland (Gibler, 2010). The stakes are frequently emphasized in game theoretic models in which resolve is reflected in an actor's payoff structure, and is thus a function of the benefits of fighting relative to the costs.

Second are strategic interests, or the reputational costs that are incurred when an actor backs down, which for sake of symmetry, we might classify as extrinsic interests instead. There is a lively debate in the IR literature as to whether reputa-

tions are formed or broken in the manner that deterrence theorists assume (Hopf, 1991b; Mercer, 1996; Huth, 1997; Tang, 2005; Tingley and Walter, 2011), but more important for our purposes is the fact that reputations are typically formed for resolve, out of the belief that an actor who backs down from a threat or surrenders in a conflict will gain the reputation of being a "weak type" and therefore be more likely to end up in costly conflict in the future (Schelling, 1966; Alt, Calvert, and 
Humes, 1988; Huth, 1997; Walter, 2006). ${ }^{6}$ Domestic audience costs also fall in this extrinsic category, in that leaders who back down in a crisis situation then have to contend with the costs of punishment imposed by the electorate or their domestic constituency (Fearon, 1994; Weeks, 2008; Hoffman et al., 2009). Thus, for all these reasons we can expect that the higher the costs of backing down, the more resolve an actor will display.

H2: The higher the costs of backing down, the more resolve actors will display.

H2a: The greater the intrinsic interests at stake, the more resolve actors will display.

$\mathrm{H} 2 \mathrm{~b}$ : The greater the reputational interests at stake, the more resolve actors will display.

Situational theories of resolve that link resolve to the costs of war also have an additional ambiguity: whose costs of war count as costly? Traditional rationalist models of IR assume existence of unitary state actors pursuing policies that minimize costs and maximize benefits. ${ }^{7}$ If we relax this assumption, however, we are faced with the question of whose costs of war and costs of backing down matter in affecting a state's resolve. After all, the cost of war is typically unevenly distributed, borne more heavily by some constituencies than others (Koch and Gartner, 2005; Levy, 2006). The choice between a conscription- and volunteer-based

\footnotetext{
${ }^{6}$ Reputations are of course also formed for reasons other than resolve: states wish to maintain reputations for honesty, for example. (Sartori, 2005)

${ }^{7}$ The accuracy and utility of the unitary actor assumption is frequently contested in IR (Allison, 1971; Keohane and Nye, 1977; Milner, 1997; Moravscik, 1997; Fearon, 1998; Hudson, 2005), but what matters for our purposes is that a divide exists in the rationalist literature between theories of resolve with unitary actors (Morrow, 1989; Morgan, 1990; Slantchev, 2005), and those that incorporate domestic politics, whether in the form of audience costs (Fearon, 1994), agent-principal problems (Downs and Rocke, 1995), selectorate theories (Bueno de Mesquita and Siverson, 1995), or two-level games (Putnam, 1988). In fact, much of the literature on the signaling of resolve explicitly requires non-unitary state actors, in that a state's ability to signal its resolve depends on the nature of the relationship between a government and its opposition (Schultz, 1998; Levy and Mabe Jr., 2004), or between the government and the public (Fearon, 1994).
} 
military, for example, has significant implications on who is "shouldering the soldiering" (Vasquez, 2005; Horowitz and Levendusky, 2011), and analyses of the effects of local casualties in the Vietnam war (Gartner, Segura, and Wilkening, 1997) suggest that the distribution of the conflict's costs also affects attitudes towards the mission in general. In addition to asymmetries in cost between different groups of the public, there also may be asymmetries between the public and its leaders; Chiozza and Goemans (2004) find that whether war is costly for leaders in terms of reduced time in office depends in large part on the regime type, such that leaders are often insulated from the costs of war imposed on their publics. Thus if a state's resolve is inversely related to the costs of war, it is important to clarify whose costs of war matter. The experimental tests carried out in Chapters 3 and 4 sidestep this level of analysis question by focusing specifically on individual-level microfoundations of resolve in the context of public opinion. I therefore defer the level

of analysis question to Chapter 5, when I use observational data to operationalize some of these situational variables both with reference to the leader (e.g. audience costs), and to the country at large.

\section{Dispositional AND INTERACTIONIST DETERMINANTS OF RESOLVE}

If casualties and the costs of backing down serve as the two situational determinants of resolve, the next task for an interactionist theory is to look for unit-level attributes or dispositional characteristics that produce resolve. We can envision dispositional determinants of resolve operating through each of the three causal pathways outlined in Table 2.1. In the main effects approach, dispositions affect resolve independently of casualties and costs of backing down, and we are interested simply in determining how much of the variation we observe in resolve is due to dispositional factors rather than situational ones. In the interactive approach, dispositions and situations have an interactive rather than an additive relationship: certain dispositions will enhance or mute the impact of casualties and the costs of backing down. Finally, the thickest interactionist approach blurs the distinction be- 
tween the two sets of independent variables, suggesting that certain dispositions affect actors' perceptions of casualties and the costs of backing down.

As suggested in the previous chapter, rational choice models of conflict have displayed a growing interest in the extent to which actors vary in their "cost sensitivity" (Slantchev, 2003; Filson and Werner, 2004; Mattes and Morgan, 2004; Filson and Werner, 2007b), but although cost sensitivity is certainly a dispositional variable, it makes for a dissatisfying explanation for resolve in that it suffers from "the problem of interiority" (Reus-Smit, 2003). That is, it explains resolve as a result of actors being less sensitive to the cost of war, but this merely pushes the causal story downwards, leaving unanswered the question of why some actors are more sensitive to the costs of war in the first place. Thus, the task I undertake here is to go beyond cost sensitivity, and point to specific specific actor-level characteristics that explain where cost sensitivity comes from. Whereas the situational determinants of resolve discussed above are specific to the study of war, the dispositional sources I look at here - time preferences, risk preferences, and honor cultures - also figure prominently in research on willpower and self-control scattered throughout the social sciences.

\section{Time preferences}

The first interactionist variable that influences which types of costs are perceived as costly is time preferences. Although there has been growing interest on time horizons in IR (Russett and Lackey, 1987; Russett et al., 1994; Barkin, 2004; Rosen, 2005; Toft, 2006; Streich and Levy, 2007), the term has been used to refer both to the length of the time window in which leaders must act (Kreps, 2008), and the idea of time preferences, a variable measuring the extent to which actors value the future compared to the present (Frederick, 2006). ${ }^{8}$

\footnotetext{
${ }^{8} \mathrm{~A}$ large body of research has been conducted on the impact of time on decision-making. We know that actors generally discount future benefits compared to present ones (Loewenstein and Elster, 1992; Elster, 2000; Frederick, 2006), although there is no consensus on whether this discounting occurs because of impulses and passions (Palacios-Huerta, 2003), evolutionary pressures in favor of immediate gratification (Moore, 1988), the implications of psychological distance (Trope and Liber-
} 
A theory of resolve based on time preferences is in many ways intuitive, since the two factors are frequently conceptualized in relation to one another. We inherently think of someone who is resolved as being "in it for the long haul"; Bénabou and Tirole $(2002,879)$ define a lack of willpower as "excessive preference for the present", Velleman (2007) portrays the will as a faculty that allows us to pursue our ends over time, Fennell $(2009,93)$ defines willpower as "an individual's efforts to resist a presently attractive course of conduct", and Ainslie $(1992,2001)$ frames the "breakdown of will" in terms of hyperbolic discounting functions (emphases added). Similarly, one of the most popular ways of modeling willpower or self-control problems in the behavioral sciences is through "dual-self" models, in which choice decisions are made as the result of a bargaining process between a present- and a future-oriented self (Thaler and Shefrin, 1981; Elster, 1986; Posner, 1997; Ainslie, 2001; Bénabou and Tirole, 2004; Fudenberg and Levine, 2006), buttressed by work in neuroscience suggesting that immediate gratification and long-term considerations activate different parts of the brain (Benhabib and Bisin, 2005). For these reasons, a main effects hypothesis (longer time horizons are associated with greater resolve) is intuitive. Suicide bombers, for example, are usually deemed resolute precisely because they have long time horizons: carrying out an attack requires sacrificing oneself in the present either to confer benefits on future generations (Azam, 2005), or in order to enjoy rewards in eternity (Toft, 2006).

And yet, we can also think of time preferences in an interactionist framework, since an actor's time preferences affect its sensitivity to costs and benefits that occur in the future relative to those that occur in the present. Decision-makers typically face costs and benefits occurring at multiple points in time. Just as choosing between carrots or cheesecake, for example, requires managing tradeoffs between present and future costs and benefits (cheesecake provides immediate gratification but negative long-term consequences, while carrots typically provide fewer benman, 2003; Eyal et al., 2004; Fujita and Roberts, 2010), or because our future selves are in fact different from our present ones and thus do not merit the allocation of our utility (Parfit, 1971). For our purposes, though, the origins of time preferences are less important than the fact that resolve has such a clear temporal component to it. 
efits in the short term, but greater benefits in the long term), ${ }^{9}$ choosing between continuing to prosecute a war or to terminate it short of victory involves a similar set of temporally-arranged tradeoffs: the human costs of war are paid upfront, whereas reputational costs are paid in the future, since as the literature on cooperation under anarchy suggests, the more actors discount the future, the less reputational costs matter (Axelrod, 1984; Oye, 1985). Thus, once actors with longer time horizons enter into a war, they should outlast those with shorter time horizons, since they should be more willing to pay the short-term human costs of war in exchange for the longer-term benefits victory is posited to bring.

H3a: Actors with longer time horizons (more patient time preferences) will display more resolve.

H3b: Actors with longer time horizons (more patient time preferences) will be relatively less sensitive to casualties and relatively more sensitive to the costs of backing down.

\section{Risk preferences}

Second are risk preferences. In IR, we frequently think of risk attitudes as a source of resolve (Morrow, 1985, 1987), and the most influential work on coercion in international politics calls coercive bargaining "competition in risk-taking" (Schelling, 1966). Given the literature equating resolve with an actor's level of "critical risk", the greatest risk of disaster a state will tolerate in order to prevail in a crisis (Jervis, 1972; Snyder and Diesing, 1977; Powell, 1988), one would expect resolve to increase with risk acceptance: the more tolerant actors are of risks, the more resolute they should be. Yet there are two reasons one might be skeptical of this hypothesized relationship. First, although IR scholars tend to see risk-acceptant behavior as an indication of a high level of resolve, sociologists have typically understood riskseeking behavior as an indication of a lack of self-control (Grasmick et al., 1993); if

\footnotetext{
${ }^{9}$ The same is true for a host of other decisions - spending versus saving, smoking versus quitting, watching television versus writing your dissertation, and so on (Gifford, 2002).
} 
resolve is indeed related to willpower and self-control, this contradiction is some cause for concern. Second, by disaggregating the costs of war it becomes apparent that both fighting and backing down can be perceived as risky; as the civil war in Iraq dragged on in 2004, neoconservatives argued that the reputational consequences of withdrawal made "cutting and running" riskier than pressing onward, whereas opponents of the war suggested that sinking into a quagmire was far riskier than a quick exit. For this reason, it is also likely that risk attitudes may not have a simple linear relationship with resolve, and may affect sensitivity to both types of costs of war.

$\mathrm{H}_{4 \mathrm{~A}}$ : Risk aversion is associated with decreased resolve. (Linear hypothesis)

$\mathrm{H}_{4 \mathrm{~B}}$ : Risk aversion has a nonlinear relationship with resolve. (Nonlinear hypothesis)

$\mathrm{H}_{4 \mathrm{C}}$ : Risk aversion increases sensitivity to both the costs of fighting, and the costs of backing down.

\section{Cultures of honor}

Third, cultures of honor offer another explanation for variation in sensitivity to different types of costs. During the era of "national character" explanations in anthropology and political science (Charlesworth, 1967; Hoebel, 1967; Morgenthau, 1985), cultural factors were commonly held to be the chief source of resolve: (Organski, 1968, 189), for example, argued that cultural identifications were a primary determinant of "national morale," while Cline (1994) argued that "cultural uniformity" was one of the main ingredients of "national will." Although these types of analyses have largely fallen out of favor, a growing body of work in both cross-cultural psychology and political science has expressed interest in cultures of honor, ideological systems that place a strong emphasis on honor or face, maintaining reputations for toughness, and standing up to perceived slights (Nisbett and Cohen, 
1996; O'Neill, 1999; Osterman and Brown, 2011). ${ }^{10}$ In the Spartan debate in his History of the Peloponnesian War,Thucydides notes that "fear, honor, and interest" are "three of the strongest motives" for war (Thucydides, 1998, I76), and this emphasis on protecting one's honor by not appearing weak in the eyes of others is manifested in what Mead (2002) refers to as the Jacksonian element in US foreign policy - traces of which are still evident in present-day neoconservatism (Haglund and Kertzer, 2008) - but it is by no means limited to the American context. Indeed, a parallel literature on political violence has noted that concerns about honor play a significant role in the mobilization of insurgents, hence the seeming intractability of many counterinsurgency conflicts (Atran, Axelrod, and Davis, 2007; Atran, 2010; Ginges and Atran, 2011).

Indeed, the psychological literature on sacred and protected values suggests that at the extreme, when principles like honor or face are endowed with sufficient significance within a belief system, actors may not be willing to entertain tradeoffs altogether (Tetlock, 2003), and will act out of a sense of duty rather than according to instrumental rationality (Ginges and Atran, 2011). If actors maintain a policy out of a sense of moral obligation completely divorced from prospects of success (Atran, Axelrod, and Davis, 2007), they display the kind of intransigence we associate with a resolute actor maintaining its policies despite inclinations to the contrary. ${ }^{11}$ If actors with high honor orientations are more likely to embrace a "cult of reputation"

\footnotetext{
${ }^{10}$ The rise of constructivism has brought renewed attention on the impact of cultural factors on international security (Johnston, 1995; Katzenstein, 1996; Desch, 1998; Haglund, 2004; Twomey, 2008; Williams, 2008), but increased interest in culture has not been restricted to constructivists: game theorists treat culture as common knowledge used to solve games with multiple equilibria (see the discussion in Wedeen, 2002), while the concept of strategic culture was coined by a realist (Snyder, 1977), and one of the most influential pieces on culture and conflict (Huntington, 1993) resists categorization.

${ }^{11}$ Rationalist models of conflict have made a similar argument, but couched in the language of issue indivisibility (Fearon, 1995; Toft, 2006): if an object under dispute is viewed as all-or-nothing, inviolable, or otherwise sacred, the lack of a bargaining range means that no amount of side payments can compensate for its loss. However, this literature has focused predominantly on indivisible parcels of territory (Hassner, 2003; Goddard, 2006) — for example, Jerusalem may be physically divisible in a literal sense, but is treated as indivisible by both Israelis and Palestinians, such that neither side is willing to entertain tradeoffs - whereas we can see it as part of a more general phenomenon, an instance of actors with heightened sensitivity to certain types of costs compared to others.
} 
(Tang, 2005) that renders them highly sensitive to the costs of backing down, it is therefore likely that they should display more resolve, and be particularly sensitive to the reputational costs of backing down.

H5a: Actors subscribing to cultures of honor will display more resolve. H5b: Actors subscribing to cultures of honor will be more sensitive to the costs of backing down.

\section{Trait self-control}

One of the rationales in looking for specific dispositional sources of resolve is the premise that when IR scholars are talking about resolve, they are actually studying the same phenomenon that social scientists in other fields refer to as willpower or self control. The psychological literature on willpower - especially the egodepletion model currently popular in psychology (Hagger et al., 2010) - tends to emphasize the extent to which willpower is non-domain specific, and is instead a individual-level characteristic activated in multiple spheres (Baumeister, Vohs, and Tice, 2007). As such, it is worth investigating whether actors who score high in what psychologists call "trait self-control" (Gibbs, Giever, and Martin, 1998; Whiteside and Lynam, 2001; Tangney, Baumeister, and Boone, 2004; Duckworth and Kern, 2011; de Ridder et al., 2012) also display more resolve in their foreign policy preferences. If trait self-control is indeed a significant predictor of resolve in military interventions, it offers further justification for linking the psychological literature on willpower with IR scholarship on resolve.

H6: Actors scoring high in trait self-control will display more resolve.

Finally, it is important to note that like their situational counterparts, these dispositional variables confront a levels of analysis question: when we model resolve as a function of dispositional characteristics like time and risk preferences, whose time and risk preferences are we talking about? Classical bargaining models in 
game theory acknowledge the possibility of unitary actors having different time horizons (Rubinstein, 1982, see also the application in Barkin, 2004), but it is also possible that leaders and publics within a state will have different time preferences. Much of the pessimism expressed by postwar realists about the instability of the foreign policy opinions of the American public stemmed from an image of the public as being as impatient as it was mercurial (Holsti, 2004), reinforced by contemporary arguments that democratic war performance declines over time as the public loses the will to fight (Bennett and Stam, 1998). It is not necessarily clear, though, that leaders will always have longer time horizons than their publics. Given the extent to which time preferences, risk preferences, and honor orientation are frequently posited to be shaped by cultural factors, domestic constructivists (e.g. Hopf (2002)) would express skepticism at the prospect that elites' characteristics are completely divorced from those of the mass public. ${ }^{12}$ Perhaps impatient publics elect impatient leaders - or, in an autocratic context, leaders with short time-horizons have an upper hand in seizing power in political environments that reward these shorter time horizons (Rosen, 2005). As is the case with the situational variables, I set this level of analysis question aside for the experimental analyses in Chapters 3 and 4 - which focus exclusively on individual-level microfoundations of resolve in members of the public - returning to it in Chapter 5 in the large-N analyses with observational data.

\footnotetext{
${ }^{12} \mathrm{~A}$ large body of research links time preferences to cultural explanations: economists frequently argue that time preferences stem from cultural factors (Rogers, 1994; Offer, 2006), while Gifford (2002) suggests that time inconsistency problems stem from a divergence between a biologicallyderived preference for immediate rewards, and cultural preferences for future ones. Classic arguments in sociology make similar claims: the Weberian notion of a "Protestant work ethic" combines both a particular cultural framework that lionizes long time horizons, as well as promotes insensitivity to certain types of costs (Weber, 1904/1984), as is also true for most forms of utopian political thought, whose appeal rests on the promise of benefits that can only be realized in the future (Noyes, 1980). Risk preferences are similarly held to have cultural origins (Weber and Hsee, 1998; Hsee and Weber, 1999), while much of the work on honor comes explicitly from cross-cultural psychology (Nisbett and Cohen, 1996; Rodriguez Mosquera, Manstead, and Fischer, 2002; Izjerman and Cohen, 2011; Leung and Cohen, 2011).
} 


\subsection{Conclusion}

I thus propose an interactionist theory of resolve that envisions it to be a function of two situational features - the cost of fighting, and the cost of backing down and three dispositional ones (time preferences, risk preferences, and honor orientations). Given that IR scholars have discussed casualties (Mueller, 1971), reputation (Mercer, 1996), time preferences (Axelrod, 1984), risk preferences (McDermott, 1998), and honor (O'Neill, 1999) before, what is new about this approach?

First, it disaggregates the costs of war, explicitly modeling the competing incentives of fighting and backing down. Much of the rational choice literature reduces resolve to the costs of war relative to the benefits - that is, an actor's payoff structure - but the framework advanced above goes a step further, investigating the complex process through which these payoffs are calculated. In this sense, the theory follows Simon's (1985) encouragement to strengthen theories of decision-making by being explicit about how actors derive their utility.

Second, it avoids the "problem of interiority" by pointing to specific dispositional characteristics to explain variations in resolve, rather than simply pointing to cost-sensitivity as a trait in and of itself. Moreover, it selects these dispositional characteristics because of the roles they play in the study of willpower and selfcontrol outside of political science, thereby attempting to understand resolve as a more general phenomenon rather than an IR-specific one.

Third, it incorporates these factors into an explicitly interactionist framework, in which these dispositional features moderate the impact of situational phenomena. Game theoretic analyses of conflict are conducive to interactionist theorizing - actors' risk preferences are incorporated into the shape of their utility functions, and time preferences incorporated into their $\delta$ parameters - but to keep models tractable, most analysts treat these parameters as fixed and target their focus elsewhere, such that resolve and "the cost of war" end up being used interchangeably. ${ }^{13}$

\footnotetext{
${ }^{13}$ Kirshner (2000), for example, points that Fearon's (1995)'s rationalist explanations for war rest on the assumption that leaders are not risk averse, or at most, risk neutral. In this sense, risk atti-
} 
The theory outlined above, however, is equally interested in both situational and dispositional phenomena, rather than assigning the former pride of place.

In the next three chapters, I test this theory at both the micro-level and at the macro-level. At the micro-level, I employ laboratory and embedded survey experiments, presenting military intervention scenarios in which I manipulate features of the situation (costs of war ex ante, costs of war ex post, and the costs of backing down), in a between-group factorial experimental design. I also measure participants' time horizons, risk preferences, honor orientations, and trait self-control using instruments developed by economists and social psychologists. At the macrolevel, I then build upon the experimental results using observational data, studying the interaction of these same variables at multiple levels of analysis in the context of great power military interventions from 1946-2003.

tudes are part of Fearon's model, but in as much as they are largely held fixed, it is difficult to see their inclusion as interactionist in spirit. 


\section{Chapter 3: Experimental Microfoundations for Resolve: I}

\subsection{Research design and materials}

To test the previously delineated hypotheses in the context of public opinion about military interventions, a laboratory experiment was conducted on 317 college students recruited from undergraduate political science classes at a large Midwestern research university in December 2011. ${ }^{1}$ The study consists of three parts: a factorial experiment that manipulates the human and reputational costs of a hypothetical military intervention, a dispositional questionnaire that measures participants' time and risk preferences, honor orientations, and trait self-control, and a concluding questionnaire that includes a battery of additional individual difference measures and demographic characteristics. Participants randomly received either the dispositional questionnaire, or the intervention scenario, first, such that the entire study can be thought of as a 2 (ex ante human costs: low or high) $\times 2$ (ex post human costs: low or high) x 2 (reputation costs: implicit or salient) $\times 2$ (order manipulation: scenario 1st or 2nd) fully-crossed factorial experimental design. The structure of the experiment is summarized in Figure 3.1, below.

\footnotetext{
${ }^{1}$ Participants - $61 \%$ of whom identified as men, $77 \%$ as white/Caucasian, $7 \%$ as having served in the US Armed Forces, and $91 \%$ as having been born in the United States - ranged in age from 17 to 59 ( $\mu$ : 21.27). Although all participants were enrolled in a political science class at the time of participating in the study, only $41.5 \%$ of them identified as political science majors.
} 


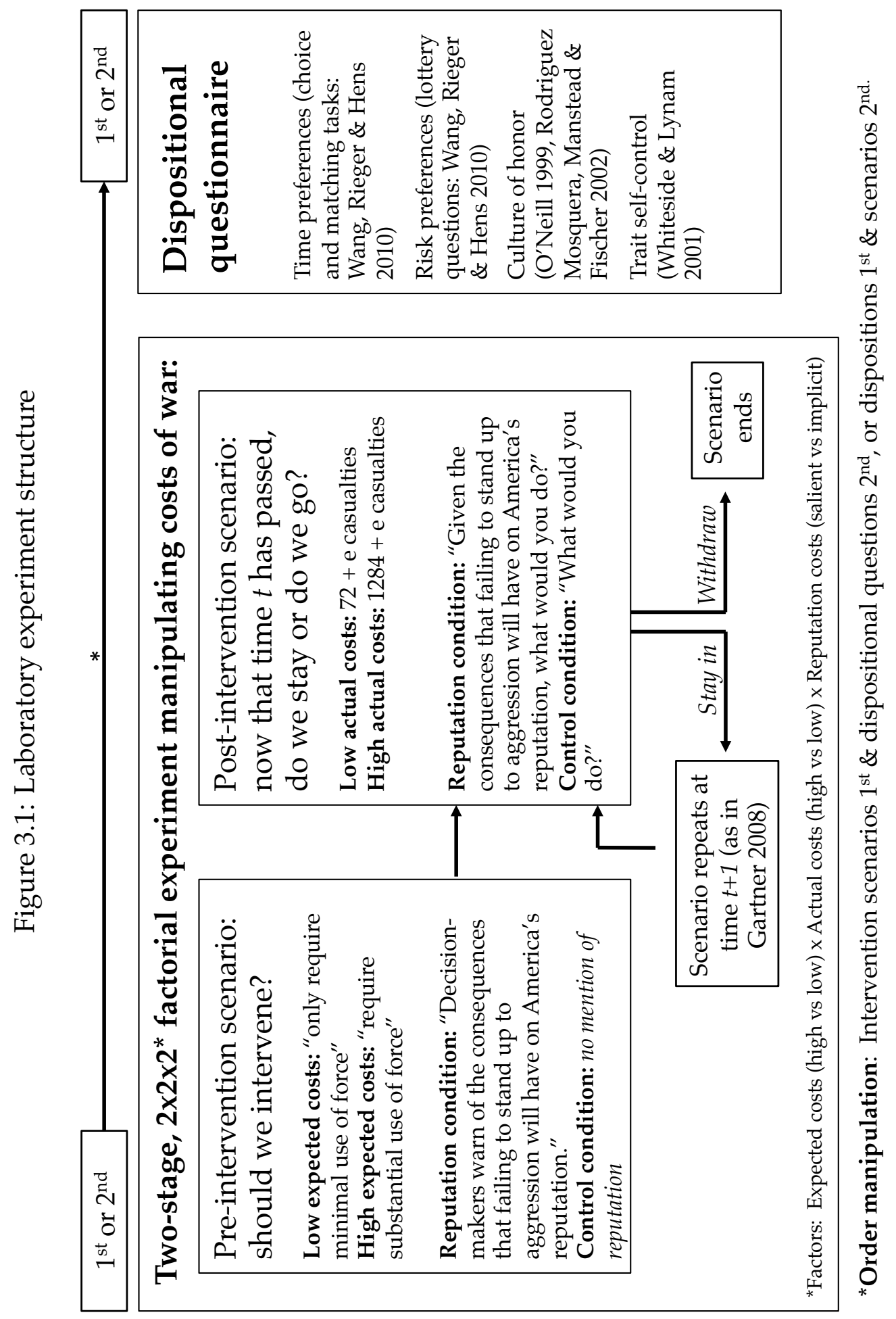




\section{SituATiONAL MANipUlations}

The experiment itself consists of a foreign policy intervention scenario that has two stages. In the first (pre-invasion) stage, participants are asked whether they support a hypothetical military intervention the President wants to carry out (modified from Herrmann, Tetlock, and Visser (1999)) on behalf of a US ally under siege. The scenario manipulates the amount of force the White House expects the intervention to require (low, or high), as well as reputational costs: in the reputation condition, participants are reminded "of the consequences that failing to stand up to aggression will have on America's reputation", while in the control condition, reputational concerns are not discussed. In the second (post-invasion) stage, I manipulate the rate of casualties the United States is experiencing (low or high), along with the same reputation manipulation described above. ${ }^{2}$

Due to the two-stage structure of the experiment, it is necessary to restrict the number of situational manipulations in order to ensure statistical power and maintain a tractable experimental design. Thus, rather than manipulating the stakes of the intervention, I keep the stakes constant and focus specifically on reputation costs as the costs of backing down. I do so for three reasons. First, reputation costs clearly correspond to a dispositional counterpart (culture of honor), allowing for the study of interactionist mechanism, in that individuals who emphasize the importance of maintaining a reputation for resolve should in theory be more sensitive to reputational concerns. Second, a large and robust literature in public opinion about foreign policy has already found evidence for the impact of the intrinsic stakes of a mission on levels of support, in that humanitarian operations are always less popular than conflicts where core national security interests are at stake (Jentleson, 1992; Klarevas, 2002), but that the popularity also depends on individuals' underlying dispositions towards humanitarian rationales for intervention

\footnotetext{
${ }^{2}$ In the low casualty condition, the US suffers $72+\epsilon$ casualties a year; in the high-casualty condition, the US suffers $1248+\epsilon$ casualties a year. These figures represent the annual equivalents of the 5th and 95th percentiles, respectively, of American monthly casualties in the Iraq War from March 2003-January 2010, with an error term $(\epsilon)$ added so that casualties fluctuate within a narrow band from year to year.
} 
(Gelpi, Feaver, and Reifler, 2009). Third, what makes resolve such an interesting dependent variable are the circumstances where we see actors behave in ways one would not expect from the stakes inherent in a situation: as social scientists, we are less interested in people who flee a fire in a crowded theater (Wolfers, 1962) than those who stay despite the fire, or who flee when no such fire exists. In this sense, it is more theoretically interesting to keep the stakes fixed and explore the impact of variation in the other situational factors. ${ }^{3}$ I return to the stakes in the large-N analyses in Chapter 5.

I employ what Gartner (2008a) calls a "panel experiment": participants receive information about how the invasion has progressed in the past year (the nature of which depends on the participants' experimental condition), and are asked whether the United States should withdraw its forces or not. If participants advocate withdrawal, the intervention scenario ends; otherwise, the process repeats up to 7 times. The dependent variable is thus the number of periods participants supported the war, analyzed using a Cox model, a semi-parametric event history model used to study survival data (Box-Steffensmeier and Jones, 2004).

\section{Dispositional MEASURES}

To measure time preferences, I employ hypothetical choice tasks commonly used by both behavioral economists who focus on time preferences and social psychologists who study self-control (Ameriks et al., 2007; Benjamin, Choi, and Strickland, 2010; Wang, Rieger, and Hens, 2010). I employ "matching" tasks, where participants are presented with a hypothetical choice between receiving $\$ 1000$ today, or $\$ X$ at some point in the future (e.g. a year from now), and asked how much $\$ X$ would have to be in order for it to be preferred over the immediate payment. By employing two different matching questions that vary the delay (e.g. one year

\footnotetext{
${ }^{3}$ I also set aside the issue of audience costs. Audience costs are by definition paid by leaders rather than ordinary citizens, but since the experimental participants are exclusively the latter, audience costs are irrelevant in this context. For good experiments investigating audience costs, see Tomz (2007); Trager and Vavreck (2011); Levendusky and Horowitz (2012).
} 
versus ten years), I have enough information to actually construct hyperbolic discounting curves for each participant, and can calculate both the value of $B$ (the participant's present bias) and $\delta$ (the participant's long-term discount factor).

I solicit risk attitudes using a series of hypothetical lottery questions employed by economists (Wang, Rieger, and Hens, 2010), in which participants are asked how much they are willing to pay for a lottery ticket with a certain chance of receiving a particular prize. The questions manipulate the value of the prize (e.g. a $60 \%$ chance of winning $\$ 100$, a $60 \%$ chance of winning $\$ 400$ ) in order to produce an estimate of participants' relative risk premium. ${ }^{4}$

There is a prominent literature on cultures of honor coming out of cross-cultural psychology (Nisbett and Cohen, 1996), but it tends to assume the existence of cultures of honor based upon ethnic categories (e.g. Latinos have a culture of honor, while the Dutch have a "culture of dignity" (Izjerman and Cohen, 2011)) or infer it from geographical origin (e.g. Presidents born in southern states, rather than those born in northern ones (Dafoe and Caughey, n.d.) rather than measure it with survey instrumentation. I thus constructed a new four-item scale measuring support for culture of honor, derived from the discussion in O'Neill (1999), and based off of items used by Rodriguez Mosquera, Manstead, and Fischer (2002).

Finally, I also use the urgency and perserverance subscales of Whiteside and Lynam's (2001) trait self-control measure to study participants' levels of trait selfcontrol. $^{5}$ I also measure the usual assortment of demographic characteristics and individual differences (party ID, political ideology, and so on).

\footnotetext{
${ }^{4}$ Although there's some evidence in the behavioral decision-making literature that risk preferences are highly context-specific (Weber, Blais, and Betz, 2002; Freese, 2010), the rationalist IR literature on risk operates out of an expected utility framework in which actors are either risk-acceptant, risk-neutral, or risk-seeking (e.g. see the debate between Fearon, 1995; Kirshner, 2000), so I follow that convention here.

${ }^{5}$ Trait self-control is generally understood to be multidimensional (Grasmick et al., 1993; Arneklev, Grasmick, and Bursik, 1999; Duckworth and Kern, 2011). Whiteside and Lynam (2001) find that trait self-control has four factors, two of which are urgency and perseverance, both of which seem plausibly related to what IR scholars mean by resolve - urgency implicating one's ability to control impulses, and perseverance referring to one's "sticktoitiveness" in carrying out challenging or ungratifying tasks. The other two factors, premediation (whether one thinks before acting), and sensation seeking (whether one is attracted to exciting or risky behavior), are less obviously related to the construct under investigation here.
} 


\subsection{Results}

The results are discussed in four phases. First, I examine the dependent variables

of interest - the extent to which participants supported the US sending troops $e x$ ante, and the duration they supported the mission ex post - demonstrating that some of the participants displayed far more resolve than others. Second, I analyze the impact of the situational manipulations to determine how participants' resolve was shaped by the human costs of fighting and the reputational costs of backing down, and how disaggregating participants based on their initial support for sending troops paints a finer-grained picture of resolve. Third, I describe my dispositional variables, and show how they are associated with resolve. Finally, I test my interactionist hypotheses, demonstrating how the impact of the situational determinants of resolve varies based upon certain dispositional features.

\section{HOW RESOLVED WERE THE PARTICIPANTS?}

As Figure 3.2 shows, there was considerable variation both in participants' initial degree of support for the intervention, and how long until they advocated for withdrawal once the US intervened. $30.5 \%$ of respondents opposed the US getting involved in the first place, compared to the $69.5 \%$ who supported deploying troops, but as the intervention progressed, the balance of support dropped precipitously: by the second anniversary of the intervention, nearly half $(46.6 \%)$ of the respondents wanted the US to leave. Two points are especially worth noting here. First, respondents displayed considerable variation in resolve: around a third (32.1\%) of participants wanted the US to cut and run by the end of its first year, whereas a fifth (20.1\%) of participants continued to support the mission even when the scenario ended in its seventh year. Since, in an event history setup, those respondents who continued to support the mission even after the scenario ended would be "rightcensored" observations, participants who persisted in supporting the mission at the end of seven years were given an open-ended question asking how many more 
years they would continue to support the intervention. Importantly, although $37.5 \%$ of the responses provided specific timelines (e.g. 13 of the 64 respondents mentioned 10 years as a suitable benchmark for withdrawal), the rest rejected the premise of the question, giving responses like "Until the job is done", "As long as it takes", "Until we win", and 'Until the mission is completed."

Figure 3.2: Distribution of the dependent variable

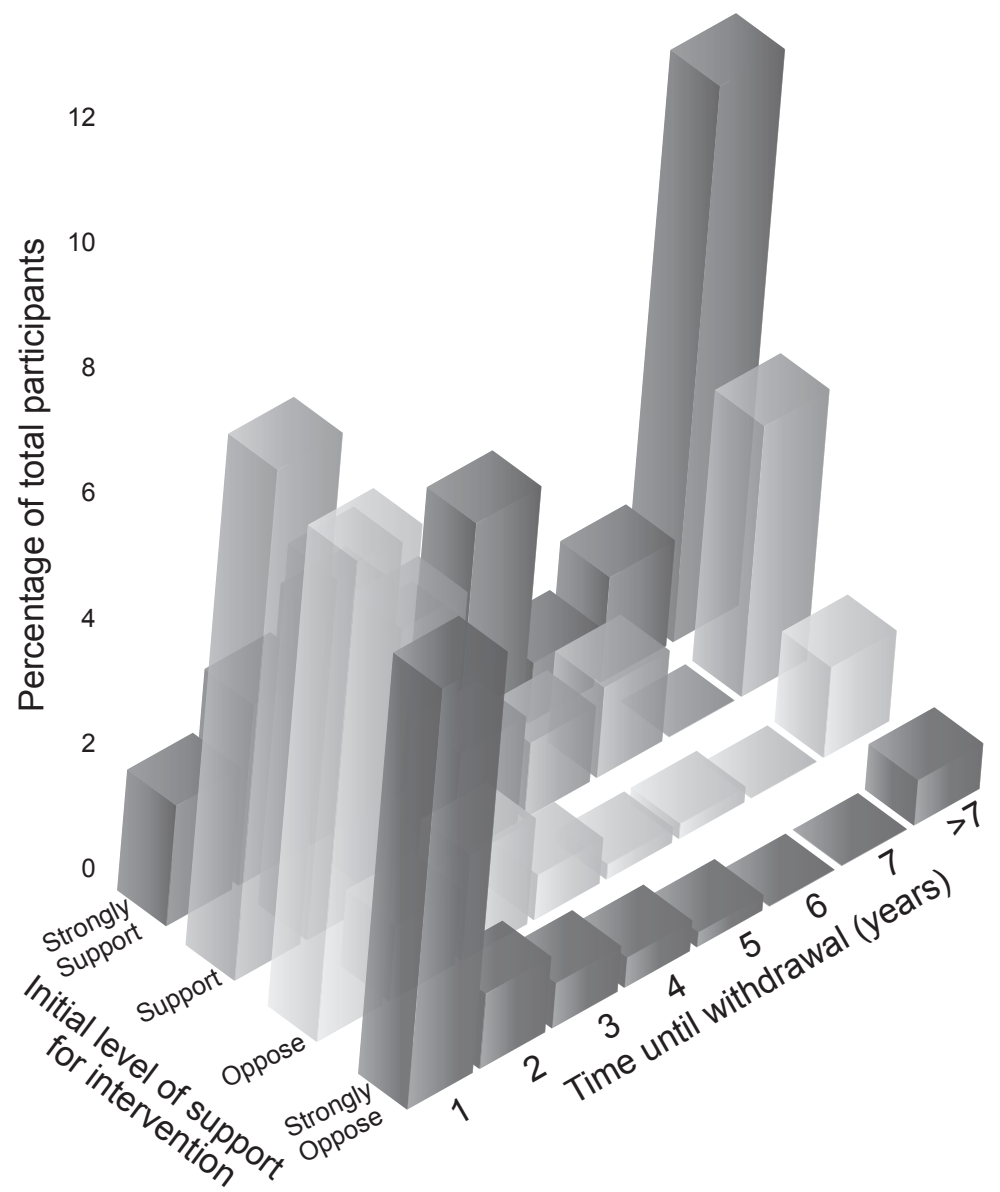

Do we go in, and how long do we stay? Initial attitudes towards the mission predict the duration of support. $\chi^{2}=104.071, p<0.000$

Second, how long people wanted US troops to stay was highly dependent on their initial attitude towards the intervention: as Figure 3.2 shows, individuals who did not want the US to get involved in the first place were far more likely to advocate an earlier withdrawal than individuals who were initially strongly supportive 
of the mission. In this sense, although we see some evidence of sunk cost logic at work - a third of the respondents who were strongly opposed to the US intervening were nonetheless willing to stick it out for at least two years, and a number of the open-ended responses include comments about how "to withdraw before success would waste the lives and efforts of those before them" - in general, participants' initial attitudes towards the mission strongly predict their attitudes towards it once troops are deployed. Thus, my findings better support Gelpi, Feaver, and Reifler's (2009) claims about how public opinion towards military operations is structured by perceptions of a conflict's rightness than they do Sullivan's (2008) arguments about sunk costs. ${ }^{6}$

\section{Situational DETERMinANTS OF RESOlve}

Thus far, the results show that some participants were highly resolved, advocating for the intervention many years after it had begun, whereas others were relatively irresolute, supporting the mission before it began but quickly favoring withdrawal as time passed. To what extent was this variation caused by the situational manipulations? Duration data displays a number of features that makes it inappropriate to study with traditional linear regression techniques: it is skewed rather than normally distributed, and more importantly for our purposes, displays right censoring: the dependent variable is the time until individuals call for troop withdrawal, but because a fifth of the participants want the mission to continue even after the time period ends, a traditional regression model would treat this part of the sample as missing, biasing coefficient estimates (Box-Steffensmeier and Jones, 2004). Instead, I employ semiparametric Cox models, as presented in Table 3.1, which, as a first

\footnotetext{
${ }^{6}$ Importantly, though, when Gelpi, Feaver, and Reifler (2009) discuss beliefs about whether a war is justified, they are invoking a retrospective justification, which, as they acknowledge, may be endogenous to their other key independent variable, beliefs about the probability of success, since individuals are likely to update their belief as to whether an intervention was the "right thing" based upon how the intervention progresses. Here, though, the belief about whether the United States should intervene is measured at the beginning of the scenario, and thus is independent of the events on the ground that follow.
} 
Table 3.1: Situational determinants of the duration of the intervention

\begin{tabular}{lcccc}
\hline & $\beta$ & SE & Hazard Ratio & $90 \%$ CI \\
\hline Anticipated costs & -0.202 & $(0.128)$ & $-18.28 \%$ & {$[-33.7 \%-0.07 \%]$} \\
Reputation costs & -0.192 & $(0.129)$ & $-17.51 \%$ & {$[-33.2 \%-1.9 \%]$} \\
Casualties & 0.277 & $(0.127)$ & $31.9 \%$ & {$[7.1 \%-62.5 \%]$} \\
Order & 0.037 & $(0.128)$ & $3.8 \%$ & {$[-15.8 \%-27.9 \%]$} \\
\hline
\end{tabular}

$\mathrm{N}=317$. Main entries are Cox model coefficients. Positive coefficients indicate a greater likelihood of 'cutting and running.'

cut, displays the results from a Cox model with each of the four treatments. ${ }^{7}$

As the hazard ratios from Table 3.1 suggest, casualties have a substantively large impact on participants' resolve, in a manner consistent with $\mathrm{H}_{\mathrm{r}}$ : when casualty levels are high, participants are $31.9 \%$ more likely to call for troops to be withdrawn at any given period of the intervention than when casualty levels are low. Interestingly, anticipated costs have the opposite effect: when participants were warned in advance that the intervention would require a substantial use of force, the respondents were $18.3 \%$ less likely to withdraw than if they were promised that the intervention would require only minimal amount of force. Cost-sensitivity thus appears to partially stem from actors' expectations: participants were more patient when the intervention had been framed in advance as requiring a substantial amount of force than when it required only a minimal use. ${ }^{8}$ In line with $\mathrm{H}_{2}$, reputation costs also bolstered actors' persistence: participants reminded of the costs of backing down were $17.5 \%$ less likely to withdraw than those who received no such reminder, thereby showing that participants were attentive both to the cost of fighting, and to the cost of backing down. These results are presented in visual

\footnotetext{
${ }^{7}$ Test results suggest no violations of the proportional hazards assumption. In lieu of presenting the higher-order interactions - which become difficult to substantively interpret, and suffer from a lack of statistical power - I focus solely on the main effects here, following the norm in largescale experimental analyses in IR (e.g. Herrmann and Shannon (2001); Tomz (2007)). Table B.4 and Figure B. 8 in Appendix B display the hazard ratios (with $90 \%$ confidence intervals) for the full $2 \times 2 \times 2 \times 2$ model.

${ }^{8}$ The expected cost manipulation may also have had this bolstering effect because participants perceived the amount of force the mission was expected to require as a proxy for how serious the stakes were for the intervention.
} 
form in the dark black lines in Figure 3.3, which depict the hazard ratio with $90 \%$ and $95 \%$ confidence intervals for each of the situational manipulations; the greater the hazard of withdrawal, the less resolve participants display. Thus, the coefficient plot show that the anticipated cost treatment and the reputation cost treatment bolster participants' resolve, while the casualty treatment lowers it, and the order manipulation has no significant effect. ${ }^{9}$ Finally, since participants remain in the same casualty treatment as the intervention progresses, one might be concerned about the possibility of demand characteristics (Orne, 1962), in which participants become aware of the purpose of the study and attempt to behave accordingly. Sup-

plementary analyses presented in Appendix B, however, suggest little concern is warranted, in that participants' predictions of future casualty rates do not become more accurate the longer they stay in the study.

\section{A more precise measure of resolve}

Table 3.2: Treatment effects based on initial decision to invade

\begin{tabular}{lcc|cc|cc}
\hline & \multicolumn{2}{c}{ All participants } & \multicolumn{2}{c}{ Go in } & \multicolumn{2}{c}{ Stay out } \\
\hline & $\beta$ & Hazard Ratio & $\beta$ & Hazard Ratio & $\beta$ & Hazard Ratio \\
\hline Anticipated costs & -0.202 & $-18.28 \%$ & -0.314 & $-26.9 \%$ & -0.175 & $-16.1 \%$ \\
& $(0.128)$ & {$[-33.7 \%, 0.07 \%]$} & $(0.158)$ & {$[-43.6 \%,-5.3 \%]$} & $(0.226)$ & {$[-42.0 \%, 21.4 \%]$} \\
Reputation costs & -0.192 & $-17.51 \%$ & -0.119 & $-11.2 \%$ & -0.122 & $-11.4 \%$ \\
& $(0.129)$ & {$[-33.2 \%, 1.9 \%]$} & $(0.162)$ & {$[-31.9 \%, 15.8 \%]$} & $(0.224)$ & {$[-38.7 \%, 27.8 \%]$} \\
Casualties & 0.277 & $31.9 \%$ & 0.294 & $34.2 \%$ & 0.187 & $20.5 \%$ \\
\multirow{2}{*}{ Order } & $(0.127)$ & {$[7.1 \%, 62.5 \%]$} & $(0.158)$ & {$[-3.6 \%, 73.7 \%]$} & $(0.215)$ & {$[-15.3 \%, 71.5 \%]$} \\
& 0.037 & $3.8 \%$ & 0.101 & $10.6 \%$ & -0.352 & $-29.7 \%$ \\
$\mathrm{~N}$ & $(0.128)$ & {$[-15.8 \%, 27.9 \%]$} & $(0.161)$ & {$[-15.0 \%, 44.0 \%]$} & $(0.217)$ & {$[-0.51 \%, 0.4 \%]$} \\
& \multicolumn{3}{c}{317} & & 220 & \\
\hline
\end{tabular}

Main entries are Cox model coefficients; SEs in parentheses; 90\% CIs around hazard ratios in brackets. Positive coefficients indicate a greater likelihood of 'cutting and running.'

Given the two-stage nature of the experimental design - in which we have measures both of participants' desire to intervene ex ante and how long they support

\footnotetext{
${ }^{9}$ I present hazard ratio plots rather than survival curves because of the former's ease of interpretability; survival curves for Table 3.1 are presented in Figure B.2 in Appendix B.
} 
Figure 3.3: Coefficient plot of situational determinants of resolve

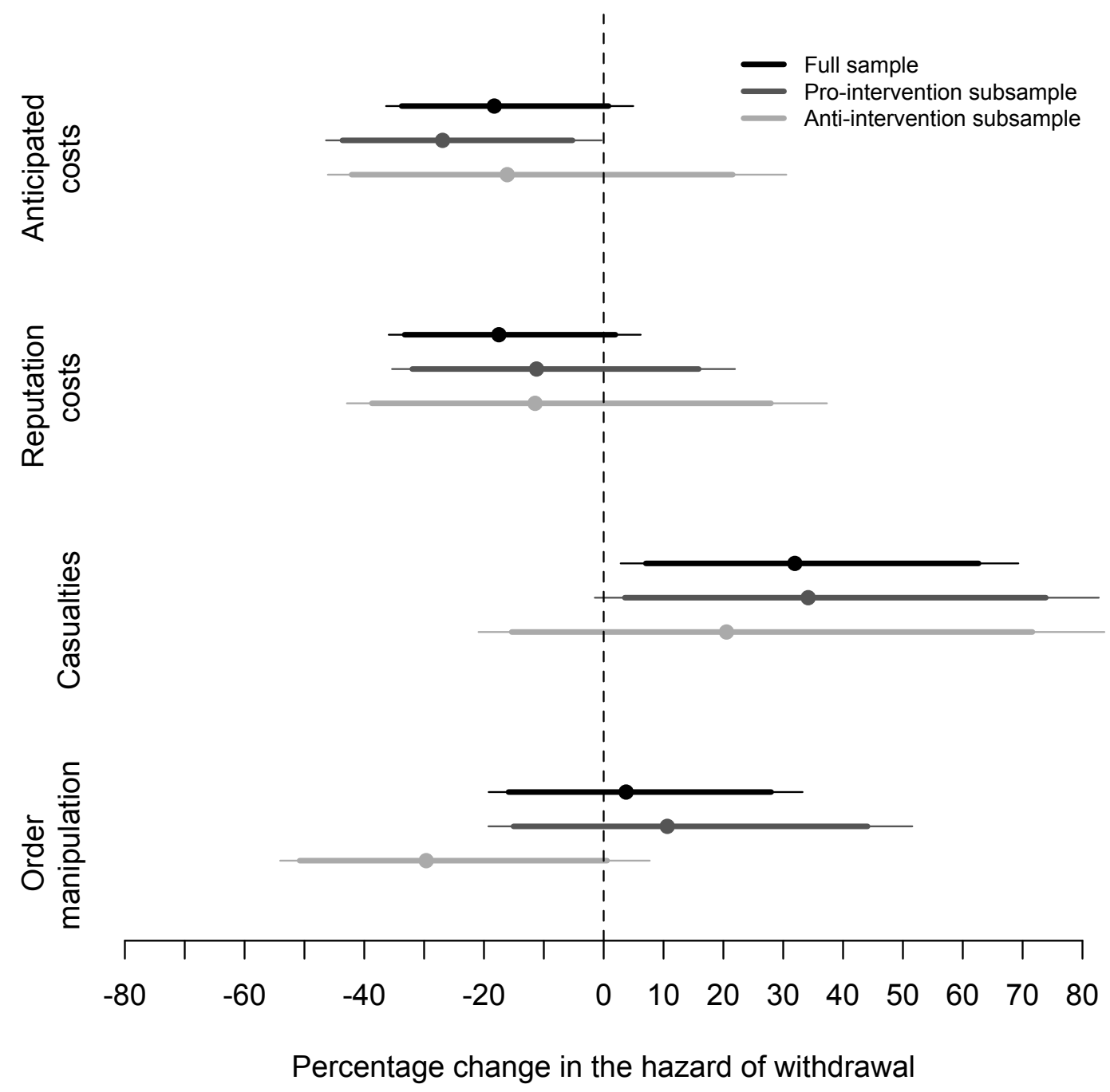

Point estimates of percentage change in the hazard ratio incurred by each of the four experimental manipulations, with 90 and $95 \%$ confidence intervals. Negative values indicate a decreased probability of withdrawal, and thus, greater resolve. 
the mission ex post, we have the opportunity to measure resolve in a manner that closely corresponds to our definition of the concept. Thus, rather than simply inferring resolve based on the length of time individuals supported the mission (deeming participants who want to immediately "cut and run" less resolute than those who want the United States to remain involved for a longer period of time), we can also look more specifically at the duration individuals who initially supported the mission continued to do so. After all, we know that resolve involves maintaining a policy despite temptations to back down, and the individuals who never supported the mission in the first place cannot be deemed irresolute for advocating for withdrawal. In a US political context, for example, Sen. John Kerry's evolving stance on the Iraq War caused him to be lampooned by his critics during the 2004 Presidential election campaign, who seized on lines like "I actually did vote for [an $\$ 87$ billion military appropriations bill] before I voted against it" as a sign that the Presidential candidate was an irresolute "flip-flopper." In contrast, Sen. Dennis Kucinich, who voted against the Iraq War Resolution in October 2002, was never in favor of the war, and thus could not be criticized for lacking resolve by continuing to oppose it.

The first model in Table 3.2 thus replicates the results from Table 3.1, while the second and third models analyze the impact of the situational manipulations for the 220 participants who initially advocated that the US intervene, and the 97 participants who initially preferred the country stay out, respectively. ${ }^{10}$ Among participants who initially advocated intervening (whose hazard ratios and confidence intervals are illustrated by the dark grey lines in Figure 3.3), both anticipated costs and casualties remain highly significant, with higher ex ante costs associated with a $26.9 \%$ increase in resolve, and higher casualties associated with a $34.2 \%$ decrease in resolve. However, the impact of reputation costs is no longer statisti-

\footnotetext{
${ }^{10}$ The decision to disaggregate the experimental results based on pre-intervention support (rather than include pre-intervention support as a control variable) stems from concerns about posttreatment bias (King and Zeng, 2007, 201-202): the treatments that shape the initial decision to intervene are also likely to affect the duration of the intervention, so controlling for the decision to intervene as an additional covariate in the model would thus bias the coefficient estimates of our treatments.
} 
cally significant, and is associated with only an $11.2 \%$ increase in resolve, although the magnitude of the change between the two models is not statistically significant. ${ }^{11}$ Although the width of the confidence intervals suggest caution should be taken in comparing treatments between the full sample and the pro-intervention subsample, ${ }^{12}$ it is nonetheless noteworthy that reputation costs are the only treatment effect whose point estimate decreases in size when only those participants who initially advocated for the intervention are studied. One potential explanation for this change are selection effects: if participants already take reputation costs into account when deciding whether to initially support the mission, it should no longer an exert an effect when deciding whether to stay or not. ${ }^{13}$

Figure 3.4: Mediation structure

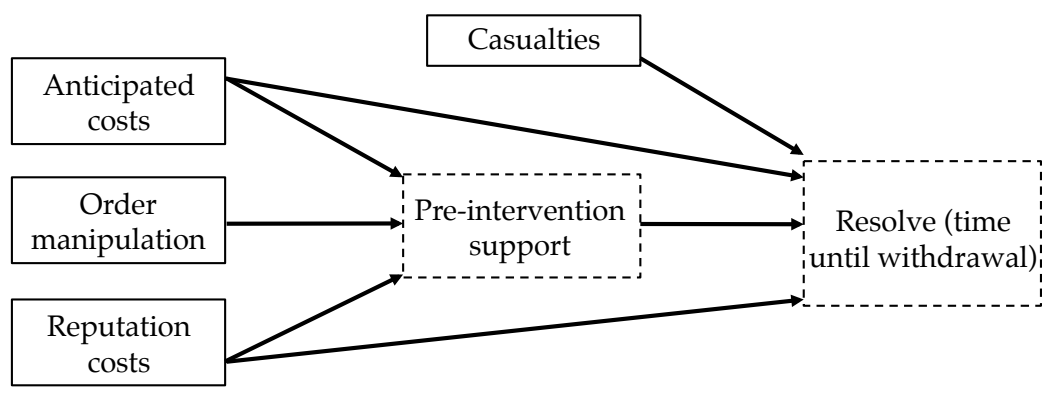

Note: the order manipulation's direct effect on resolve is omitted here for presentational purposes

Nonparametric mediation analyses offer a formal means of testing this selec-

\footnotetext{
${ }^{11}$ Both AIC scores and likelihood ratio tests comparing (i) an unrestricted model with an interaction between reputation costs and initial attitudes towards the intervention, to (ii) a restricted model without the interaction term find no significant improvement in model fit by varying the impact of reputation costs based on pro-intervention attitudes. Note that traditional tests of coefficient stability like the Chow test are inappropriate in a survival model context because they rely on the sum of squared residuals.

${ }^{12}$ For this reason, it is also difficult to judge the impact of the treatments on participants who initially advocated staying out; the smaller sample size mean that the standard errors increase in size (and thus the confidence intervals around the hazard ratios increase), but the order manipulation is noticeably statistically significant, with participants who received the intervention scenario after completing the dispositional questionnaire being $29.7 \%$ more resolved than those who answered in the other order - a similar effect as was present when analyzing support for the initial decision to intervene.

${ }^{13}$ For a series of analyses using manipulation checks to test the selection effects hypothesis alongside two alternative hypotheses - an escalation effects account, and a motivated reasoning account - see Appendix B.
} 
tion effect hypothesis, in which the impact of the reputation cost and anticipated cost manipulations on the duration of support for the mission is mediated by initial attitudes, as illustrated by Figure 3.4. Since the results from the disaggregated analyses show that reputation costs appear to exert very different effects on resolve depending on whether participants initially supported the intervention, mediation analysis is beneficial in its ability to decompose the treatment's average total effect (ATE) into two quantities of interest: the average direct effect (ADE) and average causal mediation effect (ACME) (Imai et al., 2011). ${ }^{14}$ The ACME measures how much of the treatment effect operates through the mediator - in this context, how much of the reputation cost manipulation bolsters resolve by increasing the likelihood of initially supporting US involvement in the mission. The ADE measures how much of the treatment effect operates through all other mechanisms by which the treatment might affect the outcome variable, rather than through the mediator.

The mediation analyses not only offer support for the selection effects hypothesis, but show that reputation costs and anticipated costs affect resolve through different pathways. ${ }^{15}$ The results presented in Figure 3.5 show that the impact of anticipated costs on resolve is not mediated by initial support for the mission: across all three model specifications, the ACME lacks statistical significance, and no more than $5 \%$ of anticipated costs' effect on resolve comes through the mediator. The av-

\footnotetext{
${ }^{14}$ The ATE is equivalent to the treatment effect we would estimate without mediation analysis; it is called the average total effect because in a potential outcomes framework, unit-level treatment effects are unobservable due to the fundamental problem of causal inference - that is, a unit can either be exposed to the treatment, or a control, but not both. Experimenters thus typically estimate causal effects by focusing on the average differences in the outcome variable between the treatment and control groups.

${ }^{15}$ The mediation software can only estimate parametric duration models, so in the analyses that follow I estimate a series of Weibull models rather than the Cox models estimated in the main text. The results are similar to that those from the Cox model estimated in Table 3.1: anticipated costs raise resolve by $23.0 \%$, reputation costs boost resolve by $22.3 \%$, casualties lower resolve by $33.0 \%$, and the order manipulation decreases resolve by a statistically insignificant $2.46 \%$. Although the advantage of Cox models is that they do not force analysts to make assumptions about the shape of the baseline hazard (Box-Steffensmeier and Jones, 2004, 47-48), Bennett (1999) argues that there are some instances where the functional form of duration dependence can be theoretically important. In our case, the shape parameter $(p)$ is positive $(p=1.132)$, which means the hazard is monotonically increasing with time, reflecting the tendency for participants to "lose heart" and be more likely to advocate withdrawal as time goes on.
} 
Figure 3.5: Nonparametric mediation effects: lab experiment
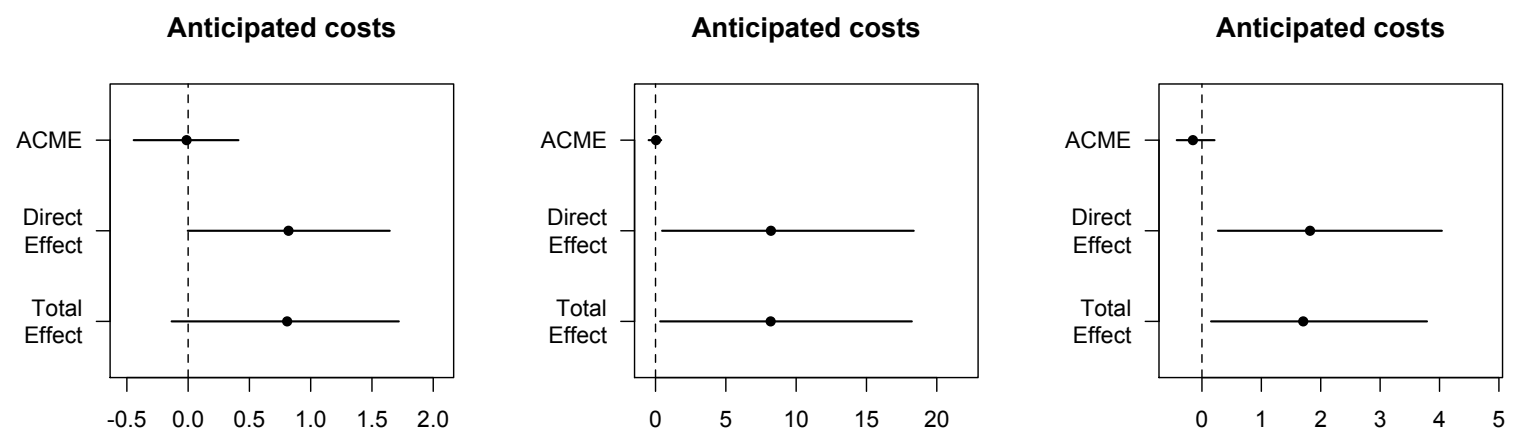

Reputation costs

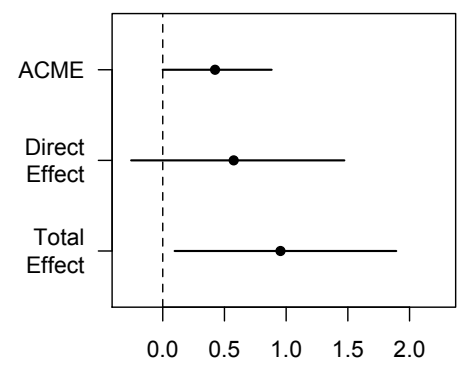

(a) No pretreatment covariates (b) Dispositional controls

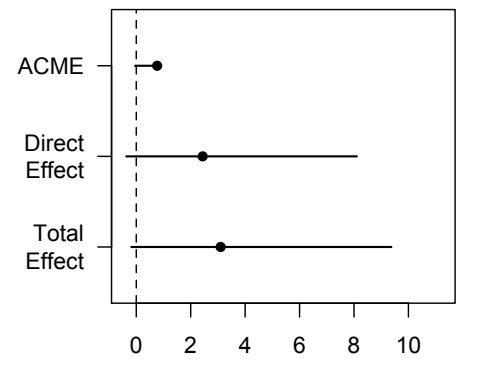

(c) Dispositional \& demographic controls

Mediation effects plotted using the mediation package from Tingley et al. (2012). All models are estimated using 1500 simulations with 90\% quasi-Bayesian confidence intervals. Anticipated costs bolster resolve by making participants more patient after the intervention takes place, while reputation costs bolster resolve by making participants more likely to approve of the mission in the first place. Because the software cannot currently conduct sensitivity analyses on duration data, I run three iterations of the analyses: first, on a model with no pretreatment covariates, second, controlling for the dispositional factors of interest, and third, controlling for both the dispositional variables and demographic characteristics. I thus cannot eliminate the possibility that the results are an artifact of pre-treatment confounders, but I can test how robust they are to the inclusion of a large number of pre-treatment controls. 
erage direct effect of anticipated costs, on the other hand, is positive and statistically significant, and increases in significance (to $p<0.01$ ) when pretreatment covariates are introduced. It is thus clear that anticipated costs raise resolve, but not by raising the likelihood of supporting the intervention ex ante. Instead, being warned in advance that an intervention is expected to require substantial use of force appears to make participants more patient when the US does in fact encounter obstacles.

In contrast, the reputation cost manipulation displays a borderline statistically significant ACME ( $p<0.1$ with no pretreatment covariates, $p<0.13$ with demographic characteristics), yet no significant $\operatorname{ADE}(p<0.23$ to $p<0.30$, depending on the model specification). In a model with dispositional pretreatment covariates, a full $40.8 \%$ of reputation costs' effect on resolve goes through the mediator (although this percentage drops to $27.7 \%$ once demographic characteristics are also being controlled for). These results offer further support for the selection effects hypothesis, in that reputation costs bolster resolve by increasing the likelihood of wanting to intervene in the first place. In this respect, reputation costs and anticipated costs both exert a similar effect on resolve, but through opposite pathways: whereas anticipated costs bolster resolve by making participants more patient, reputation costs do not affect participants' patience, but rather, their desire to intervene in the first place.

\subsection{Dispositional determinants of resolve}

To measure participants' time preferences, participants were presented with two matching questions taken from Wang, Rieger, and Hens (2010), where they were given the choice between receiving $\$ 100$ today, or $\$ X$ at either 1 or 10 years in the future, and asked to indicate how much $\$ \mathrm{X}$ would have to be to make it preferable to the immediate payment. The lower the discount rate, the more the participant values the future relative to the present; an individual with a discount rate of 0 is indifferent between a payment today and an equal payment at time $t+1$, whereas an individual with a discount rate of 5 values a payment at time $t+1$ five times less 
than an equal payment today.

If participants discounted time in the manner predicted by classical (exponential) theories of discounting, they would display identical discount rates regardless of whether $\$ X$ was being offered 1 year from now, or 10 years from now. We can derive these discount rates ( $R_{1}$ and $R_{10}$, respectively) using equation 3.1 below, where $F$ is the future value, $P$ represents the present value, and $\mathrm{t}$ the time to be waited:

$$
F=P(1+R)^{t}
$$

Since $P=100$ and $F=X$, we can rearrange to solve for $R$ for both $t=1$, and $t=10$ :

$$
\begin{array}{r}
R=\left(\frac{X}{100}\right)^{t}-1 \\
R_{1}=\frac{X}{100}-1 \\
R_{10}=\left(\frac{X}{100}\right)^{0.1}-1
\end{array}
$$

Although $R_{1}$ and $R_{10}$ are correlated with one another $(r=0.502)$, participants tended to display a steeper discount rate for the 1 year payment than the 10 year one, consistent with behavioral economists' research on present bias: for $91.8 \%$ of participants, $R_{1}$ was greater than $R_{10}$, suggesting that respondents were disproportionately more impatient towards the value of the 1-year delayed reward than the 10-year delayed one. Thus, rather than assume a constant discounting rate, I estimate quasi-hyperbolic discounting functions, which model the extent to which people are more patient in the long-run than in the more immediate future, as expressed by equation 2 below, where $\beta$ is a measure of present bias, and $\delta$ a measure of long-term discounting.

$$
u\left(x_{0}, x_{1} \ldots x_{T}\right)=u\left(x_{0}\right)+\sum_{t=1}^{T} \beta \delta^{t} u\left(x_{t}\right)
$$


Both $\delta$ and $\beta$ can therefore be estimated using the values of $X$ respondents provided in the two matching questions, as shown in equations 3 and 4:

$$
\begin{array}{r}
\delta=\left(\frac{X_{1}}{X_{10}}\right)^{1 / 9} \\
\beta=\frac{100}{\delta X_{1}}
\end{array}
$$

A similar procedure was used to measure participants' risk preferences, using instrumentation from Wang, Rieger, and Hens (2010). Participants were presented with two hypothetical lottery questions in which they were given the option of winning a particular amount of money, and asked how much they would be willing to pay for a lottery ticket to play in the lottery. To measure risk aversion, participants were asked how much they would be willing to pay to buy a ticket in a lottery with (i) a $60 \%$ chance to win $\$ 100$ but otherwise nothing, and (ii) a $60 \%$ chance to win $\$ 400$ but otherwise nothing. Risk aversion was measured by averaging over the relative risk premium for each question, calculated by subtracting the expected value of the lottery from from the respondent's willingness to pay for a ticket, and dividing the difference by the expected value. ${ }^{16}$

$$
\begin{aligned}
R R P_{100}= & \frac{(0.6 * 100)-\lambda_{100}}{(0.6 * 100)} \\
& =\frac{60-\lambda_{100}}{60} \\
R R P_{400}= & \frac{(0.6 * 400)-\lambda_{400}}{(0.6 * 400)} \\
& =\frac{240-\lambda_{400}}{240}
\end{aligned}
$$

Each of these measures paint a similar picture of the participants as being overwhelmingly risk averse; of the 316 participants who completed the risk lottery

\footnotetext{
${ }^{16}$ An alternative measure of risk aversion was calculated using the Arrow-Pratt measure of absolute risk aversion (Hartog, Ferrer-i Carbonell, and Jonker, 2002), in which $\alpha$ is the probability of winning the lottery, $Z$ the value of the prize, and $\lambda$ the participant's willingness to pay, thus: $\rho=$ $\frac{\alpha Z-\lambda}{\frac{\lambda^{2}}{2}+\frac{\alpha Z 2}{2}-\alpha \lambda Z}$. Since only the scale of the denominator differs between the Arrow-Pratt measure and the relative risk premium, the results in the models that follow are quite similar regardless of which measure is used.
} 
questions, only one was truly risk acceptant $(\rho<0)$, five were risk neutral $(\rho=0)$, and the remaining 310 were risk averse $(\rho>0)$. Prospect theory finds that individuals in the domain of gains are more risk averse than in the domain of losses (Kahneman and Tversky, 2000), so in this sense, the skewed distribution of risk preferences is sensible. It is also reassuring given that prominent rationalist theories of war assume that actors are risk-averse or risk-neutral (Fearon, 1995). Because of the distribution of risk preferences among participants, the relationship between risk preferences and resolve in the analyses that follow is thus a matter of exploring degrees of risk aversion, rather than examining the entire risk spectrum.

Honor orientations were measured using a four-item scale, where participants were asked the extent to which they agree or disagree with the statements "It's always important to be true to your word", "A man should be willing to defend himself if he's insulted in public", "I think many people don't care enough about their reputation, and "People will take advantage of you if you don't stand up for yourself." 17

Finally, the dispositional questionnaire included the "urgency" and "perseverance" subscales of Whiteside and Lynam's (2001) trait self-control model. Each subscale contains four items, with urgency measuring the extent to which participants agreed or disagreed with the statements "I have trouble controlling my impulses", "When I am upset I often act without thinking", "I have trouble resisting my cravings (for food, cigarettes, etc.), and "It is hard for me to resist acting on my feelings." Perseverance measures the extent to which participants agreed or disagreed with the statements "I generally like to see things through to the end", "I tend to give up easily", "Unfinished tasks really bother me", and "Once I start a project, I almost always finish it." Both trait self-control subscales were reliable (urgency: $\alpha=0.735$ ), perseverance: $\alpha=0.708)$.

Table 3.3 shows the estimates of two Cox models that control not just for the

\footnotetext{
${ }^{17}$ The honor measure has relatively low reliability $(\alpha=0.511)$, potentially suggesting that the construct has multiple dimensions, although principal axis factoring with oblimin rotation suggests the four-item measure has only one factor according to the Kaiser criterion.
} 
Table 3.3: Dispositional and situational determinants of resolve (1)

\begin{tabular}{|c|c|c|c|c|}
\hline & \multicolumn{2}{|c|}{ All participants } & \multicolumn{2}{|c|}{ Pro-intervention participants } \\
\hline & $\mathrm{B}$ & Hazard Ratio & B & Hazard Ratio \\
\hline \multirow[t]{2}{*}{ Anticipated costs } & -0.296 & $-25.6 \%$ & -0.409 & $-33.5 \%$ \\
\hline & $(0.135)$ & {$[-40.4 \%,-7.1 \%]$} & $(0.167)$ & {$[-49.4 \%,-12.7 \%]$} \\
\hline \multirow[t]{2}{*}{ Reputation costs } & -0.214 & $-19.2 \%$ & -0.174 & $-16.0 \%$ \\
\hline & $(0.135)$ & {$[-35.2 \%, 0.7 \%]$} & $(0.172)$ & {$[-36.6 \%, 11.4 \%]$} \\
\hline \multirow[t]{2}{*}{ Casualties } & 0.250 & $28.3 \%$ & 0.280 & $32.3 \%$ \\
\hline & $(0.135)$ & {$[2.8 \%, 60.2 \%]$} & $(0.169)$ & {$[0.1 \%, 74.6 \%]$} \\
\hline \multirow[t]{2}{*}{ Order } & -0.007 & $-0.7 \%$ & 0.072 & $7.5 \%$ \\
\hline & $(0.133)$ & {$[-20.1 \%, 23.4 \%]$} & $(0.166)$ & {$[-18.5 \%, 41.7 \%]$} \\
\hline \multirow[t]{2}{*}{$\delta$} & -1.072 & $-65.8 \%$ & -1.419 & $-75.8 \%$ \\
\hline & $(0.544)$ & {$[-86.0 \%,-16.5 \%]$} & $(0.637)$ & {$[-91.5 \%,-31.2 \%]$} \\
\hline \multirow[t]{2}{*}{$\beta$} & -0.142 & $-13.2 \%$ & -0.331 & $-28.2 \%$ \\
\hline & $(0.171)$ & {$[-34.5 \%, 14.9 \%]$} & $(0.217)$ & {$[-49.7 \%, 2.4 \%]$} \\
\hline \multirow[t]{2}{*}{ Honor culture } & -1.134 & $-67.8 \%$ & -0.842 & $-56.9 \%$ \\
\hline & $(0.633)$ & {$[-88.6 \%,-9.2 \%]$} & $(0.790)$ & {$[-88.2 \%, 57.4 \%]$} \\
\hline \multirow[t]{2}{*}{ Risk aversion } & 2.556 & * & 2.404 & * \\
\hline & (1.426) & * & (1.685) & * \\
\hline \multirow[t]{2}{*}{ Risk aversion $^{2}$} & -2.491 & * & -2.554 & * \\
\hline & (1.146) & * & (1.389) & * \\
\hline $\mathrm{N}$ & \multicolumn{2}{|r|}{292} & \multicolumn{2}{|r|}{202} \\
\hline
\end{tabular}

four situational treatments, but also for participants' time preferences (both $\delta$ and $\beta)$, risk aversion, and honor orientations. ${ }^{18}$ The first model analyzes the impact of these dispositional factors on all subjects, regardless of their initial stance towards the intervention, while the second model only analyzes those subjects who initially wanted to intervene. In support of $\mathrm{H}_{3} \mathrm{~A}$, participants with more patient time preferences as measured by their long-term discounting factor $(\delta)$ displayed more resolve: an increase in $\delta$ from 0 to 1 - corresponding with a change from completely discounting future costs, to treating future costs as equivalent to present costs decreases the likelihood of withdrawal by $65.8 \%$. The more participants value the

\footnotetext{
${ }^{18}$ Importantly, none of the dispositional measures displayed significant interaction effects with the order manipulation, suggesting that we can think of these measures as reflecting actual dispositions rather than responses to the intervention scenario.
} 
Table 3.4: Dispositional and situational determinants of resolve (2)

\begin{tabular}{|c|c|c|c|c|}
\hline & \multicolumn{2}{|c|}{ All participants } & \multicolumn{2}{|c|}{ Pro-intervention participants } \\
\hline & $\mathrm{B}$ & Hazard Ratio & $\mathrm{B}$ & Hazard Ratio \\
\hline \multirow[t]{2}{*}{ Anticipated costs } & -0.280 & $-24.4 \%$ & -0.407 & $-33.5 \%$ \\
\hline & $(0.133)$ & {$[-39.2 \%,-5.9 \%]$} & $(0.165)$ & {$[-49.2 \%,-12.8 \%]$} \\
\hline \multirow[t]{2}{*}{ Reputation costs } & -0.184 & $-16.8 \%$ & -0.170 & $-15.6 \%$ \\
\hline & $(0.133)$ & {$[-33.2 \%, 3.5 \%]$} & $(0.168)$ & {$[-35.9 \%, 11.2 \%]$} \\
\hline \multirow[t]{2}{*}{ Casualties } & 0.269 & $30.9 \%$ & 0.317 & $37.3 \%$ \\
\hline & $(0.132)$ & {$[5.4 \%, 62.6 \%]$} & $(0.165)$ & {$[4.7 \%, 80.0 \%]$} \\
\hline \multirow[t]{2}{*}{ Order } & -0.006 & $-0.6 \%$ & 0.100 & $10.5 \%$ \\
\hline & $(0.132)$ & {$[-20.0 \%, 23.4 \%]$} & $(0.167)$ & {$[-16.0 \%, 45.4 \%]$} \\
\hline \multirow[t]{2}{*}{$\delta$} & -0.822 & $-56.0 \%$ & -1.166 & $-68.8 \%$ \\
\hline & $(0.529)$ & {$[-81.5 \%, 4.7 \%]$} & $(0.637)$ & {$[-89.0 \%,-11.4 \%]$} \\
\hline \multirow[t]{2}{*}{$\beta$} & -0.104 & $-9.9 \%$ & -0.324 & $-27.6 \%$ \\
\hline & $(0.168)$ & {$[-31.6 \%, 18.8 \%]$} & $(0.215)$ & {$[-49.2 \%, 3.0 \%]$} \\
\hline \multirow[t]{2}{*}{ Honor culture } & -1.065 & $-65.5 \%$ & -0.523 & $-40.7 \%$ \\
\hline & $(0.032)$ & {$[-87.8 \%,-2.4 \%]$} & $(0.782)$ & {$[-83.6 \%, 113.9 \%]$} \\
\hline \multirow[t]{2}{*}{ Perseverance } & -0.501 & $-39.4 \%$ & -1.686 & $-81.5 \%$ \\
\hline & $(0.637)$ & {$[-78.7 \%, 72.1 \%]$} & $(0.858)$ & {$[-95.5 \%,-24.4 \%]$} \\
\hline $\mathrm{N}$ & & 292 & & 202 \\
\hline
\end{tabular}

future, the more likely they are to stick it out rather than cut and run. In line with $\mathrm{H}_{5} \mathrm{~A}$, honor orientations are also statistically significant, and produce a similar effect: moving from the lowest to highest level of honor culture corresponds with a 67.8\% decrease in the likelihood of cutting and running. Risk aversion displays a significant effect, but against $\mathrm{H}_{4 \mathrm{~A}}$ and in support of $\mathrm{H}_{4 \mathrm{~B}}$, its impact on resolve is nonlinear: participants with a moderate degree of risk aversion $(\rho=0.5)$ are $92.5 \%$ more likely to withdraw than risk-neutral participants $(\rho=0)$, but past this point of risk aversion, participants actually become more resolute: a jump in risk aversion from $(\rho=0.5)$ to $(\rho=1)$ is associated with a $44.6 \%$ decrease in the likelihood of withdrawal, as illustrated by Figure 3.6. ${ }^{19}$

\footnotetext{
${ }^{19}$ To test for nonlinearity without imposing a particular functional form, risk aversion was estimated with a penalized smoothing spline with the number of degrees of freedom determined by AIC scores; the resulting model appeared to be quadratic, and a series of likelihood ratio tests confirms that the spline fails to better fit the data than a simple quadratic $\left(\mathrm{X}^{2}=0.092, p<1.000\right)$, and
} 
Figure 3.6: Quadratic effect of risk aversion ( $\mathrm{N}=2500$ simulations)

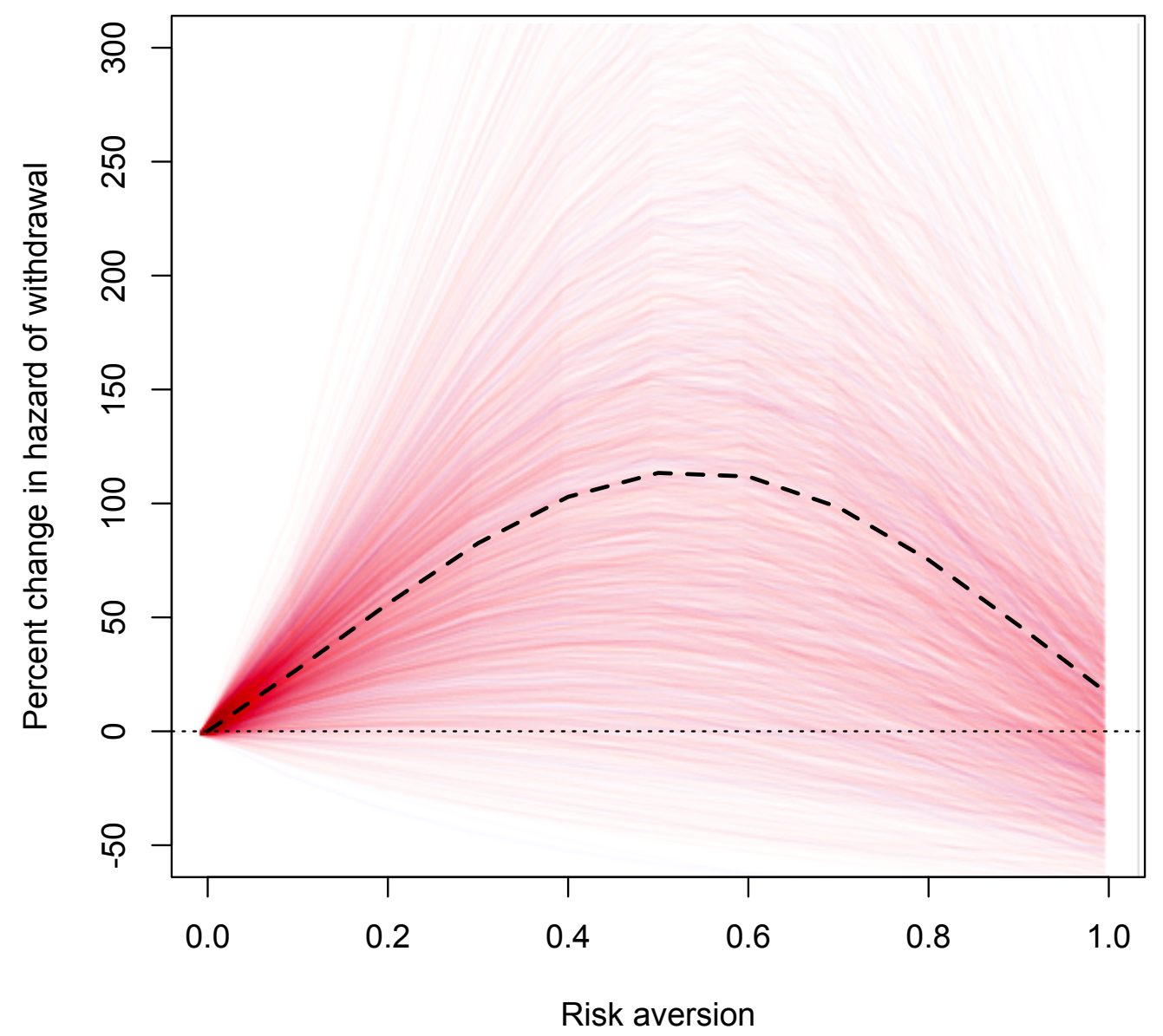

The quadratic effect of risk aversion on participants' resolve, where each red line depicts a draw from a multivariate normal distribution, given $\widehat{\beta}$ and $\widehat{v a r}(x)$ from model 1 of Table 3.3, such that darker lines indicate a more concentrated probability mass. The dashed black line represents the mean prediction. 
The second model replicates these analyses, but only for those subjects who initially advocated intervening. Reflecting the selection effect hypothesis suggested by the mediation analysis, reputation costs and honor orientations drop well out of the range of statistical significance, and as the right-hand panel of Figure B.4 in Appendix B shows, the impact of risk aversion is much the same as in the full sample (participants with a moderate degree of risk aversion $(\rho=0.5)$ are $75.7 \%$ more likely to withdraw than those who are risk-neutral, and the extremely risk averse $(\rho=1)$ are $51.0 \%$ less likely to withdraw than those with moderate risk aversion) but the impact of long-term discounting increases: a jump in $\delta$ from 0 to 1 decreases the likelihood of withdrawal by $75.8 \%$. Similarly, $\beta$ acquires borderline statistical significance: an increase in $\beta$ from 0 to $1-$ which is substantively equivalent to participants becoming more patient, as a quasi-hyperbolic discounting model is equivalent to an exponential discounting model when $\beta=1-$ is associated with a $28.2 \%$ decrease in the likelihood of withdrawal.

I therefore find support for the existence of dispositional determinants of resolve: both time preferences, honor orientations, and risk attitudes have substantively important impacts on the probability of individuals choosing to 'cut and run' in the military intervention, and in sensible directions. The multiple model specifications show that reputation and honor considerations weigh more heavily in the full sample than in the pro-intervention subsample, while time preferences display the opposite effect, increasing in both statistical and substantive significance in the pro-intervention subsample. One potential explanation for this pattern is that honor orientation, as the dispositional counterpart of the reputation cost manipulation, bolsters resolve as the reputation cost treatment does, by increasing initial

that the quadratic strongly outperforms a linear fit $\left(\mathrm{X}^{2}=11.313, p<0.001\right)$. Penalized smoothing splines were also added to other predictors in the model to test for nonlinearity, but in all cases failed to improve model fit. As a robustness check, the results were also reanalyzed without the one risk-acceptant participant in the sample, but the quadratic form of risk aversion remains. Table 3.3 was also estimated using loss aversion parameters - which measure risk attitudes in the domain of losses rather than in the domain of gains - but even with the use of a penalized smoothing spline to correct for the variable's leptokurtic distribution, the variable always fails to achieve statistical significance. 
support for the mission. Indeed, controlling for the situational manipulations and a host of demographic variables, participants high in honor were $26.6 \%$ more likely to strongly support intervening than those low in honor orientations. Thus, it follows that when only those participants who initially supported the intervention are being studied, the effect of honor should dissipate compared to the full sample. In contrast, time preferences have no significant impacts on the initial question of intervening, since the question of whether the US should intervene lacks the clear temporal dynamics of the question of how long the US should stay. The nonlinear impact of risk aversion is notable because political scientists often assume risk attitudes to display a more conventional linear effect (e.g. Kam and Simas, 2010; Eckles and Schaffner, 2011). When Schelling (1966) refers to crisis bargaining as "competition in risk-taking," the tacit assumption is that resolve should be inversely related to risk aversion, presumably in a linear fashion. However, just as war has many costs, it also has many risks: both withdrawing and persisting may be perceived as risky choices, depending upon one's frame of reference, and given the presence of these countervailing effects, a nonlinear relationship is likely.

Although participants' scores on the urgency subscale had no significant relationship with the resolve they displayed in the intervention scenario, perseverance had an effect that is both statistically significant and substantively interesting. Table 3.4 replicates Table 3.3, but controls for participants' perseverance scores rather than risk preferences - the extent to which participants reported being resolute in their daily lives, finishing tasks they start, seeing things through, tending not to give up, and so on. ${ }^{20}$ As the coefficient plots illustrated in Figure 3.7 show, although perseverance has no significant impact on the full sample, it has a highly significant effect among those participants who initially supported the intervention, associated with an $81.5 \%$ decrease in the probability of withdrawing at any given point in time. In other words, more persistent individuals also displayed greater persistence in the military intervention - but only if they approved of go-

${ }^{20}$ The model displays very similar results when both perseverance and risk preferences are included, although the statistical significance weakens. 
ing in in the first place. The implications here are threefold. First, the fact that the perseverance measures were obtained in an apolitical domain means that we have evidence of resolve as a general phenomenon rather than a domain-specific one, similar to findings in psychology (e.g. Baumeister, Vohs, and Tice, 2007). Second, just as how resolve was defined as a steadfastness of purpose, the results show that participants cannot be deemed to lack perseverance when they oppose US troops going in, and then are quick to recommend they withdraw. Instead, perseverance has an impact on the duration of the mission only among participants who supported going in in the first place. Third, the results suggest a qualification of $\mathrm{H6}$, in that although trait self-control is positively related to resolve, resolve is related to one dimension of trait self-control in particular - perseverance - rather than the construct as a whole.

Finally, a number of supplementary analyses were conducted controlling for a wide range of demographic characteristics to probe the robustness of the findings; these results are reported in Table B.3 in Appendix B.

\section{INTERACTIONIST ANALYSES}

Thus far, the analyses have shown that resolve is both situational and dispositional: it is affected both by the human costs of war and the reputational costs of backing down, as well as time preferences, risk preferences, perseverance, and to a lesser extent, honor orientations. The question becomes whether, as hypothesized, these dispositional characteristics moderate the impact of situational features. The findings presented below offer support for some of the interactionist hypotheses, but not for others: in particular, against $\mathrm{H}_{5} \mathrm{~B}$, honor orientations have no significant interaction effects with the costs of backing down. However, in support of $\mathrm{H}_{3}$ в and $\mathrm{H}_{4} \mathrm{c}$, time preferences and risk aversion display a number of significant interaction effects with the situational manipulations. 
Figure 3.7: Coefficient plot of situational and dispositional correlates of resolve

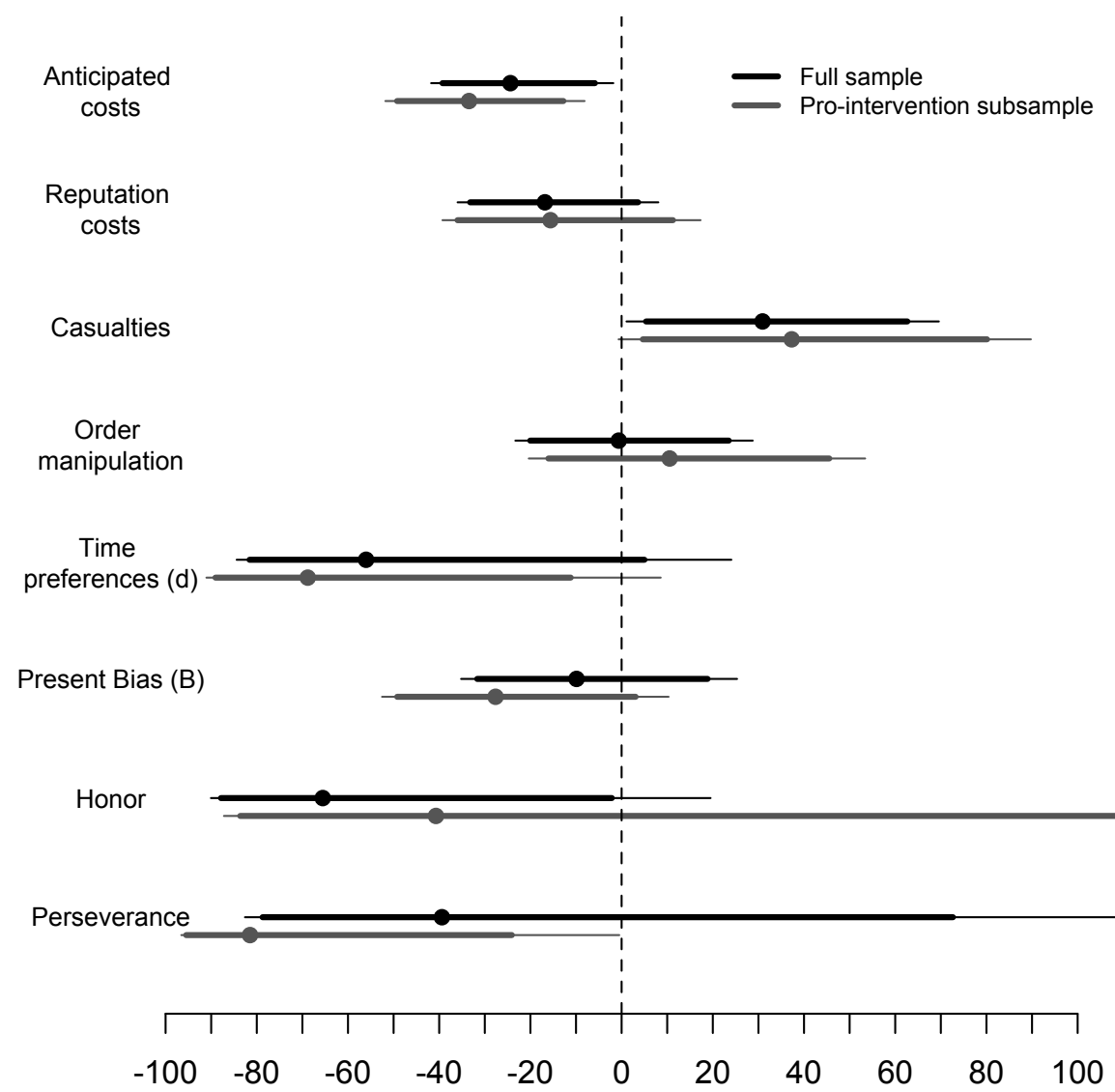

Percentage change in the hazard of withdrawal

Point estimates of percentage change in the hazard ratio incurred by moving from a 1-unit change in each variable, with 90 and $95 \%$ confidence intervals. Negative values indicate a decreased probability of withdrawal, and thus, greater resolve.

\section{Time preferences}

It was hypothesized in $\mathrm{H}_{3}$ в that time preferences would moderate the impact of the costs of war on actors' resolve: since reputation costs are predominantly paid in the future while casualties are principally paid in the present, it was hypothesized that the longer participants time preferences were - that is, the greater their longterm discounting (as measured by $\delta$ ) and lower their present bias (as measured by $\beta$ ), the more sensitive they would be to reputational costs, and less sensitive they would be to casualties. Participants' $\delta$ parameters, although highly statistically 
significant as main effects in all of the dispositional models estimated in Tables 3.3-B.3, have no significant interaction effects with the situational manipulations. Long-term discounting, reputation costs, and the human costs of war thus appear to operate simultaneously, but in an additive rather than an interactive relationship.

Figure 3.8: Time preferences $x$ casualties combined coefficient plot

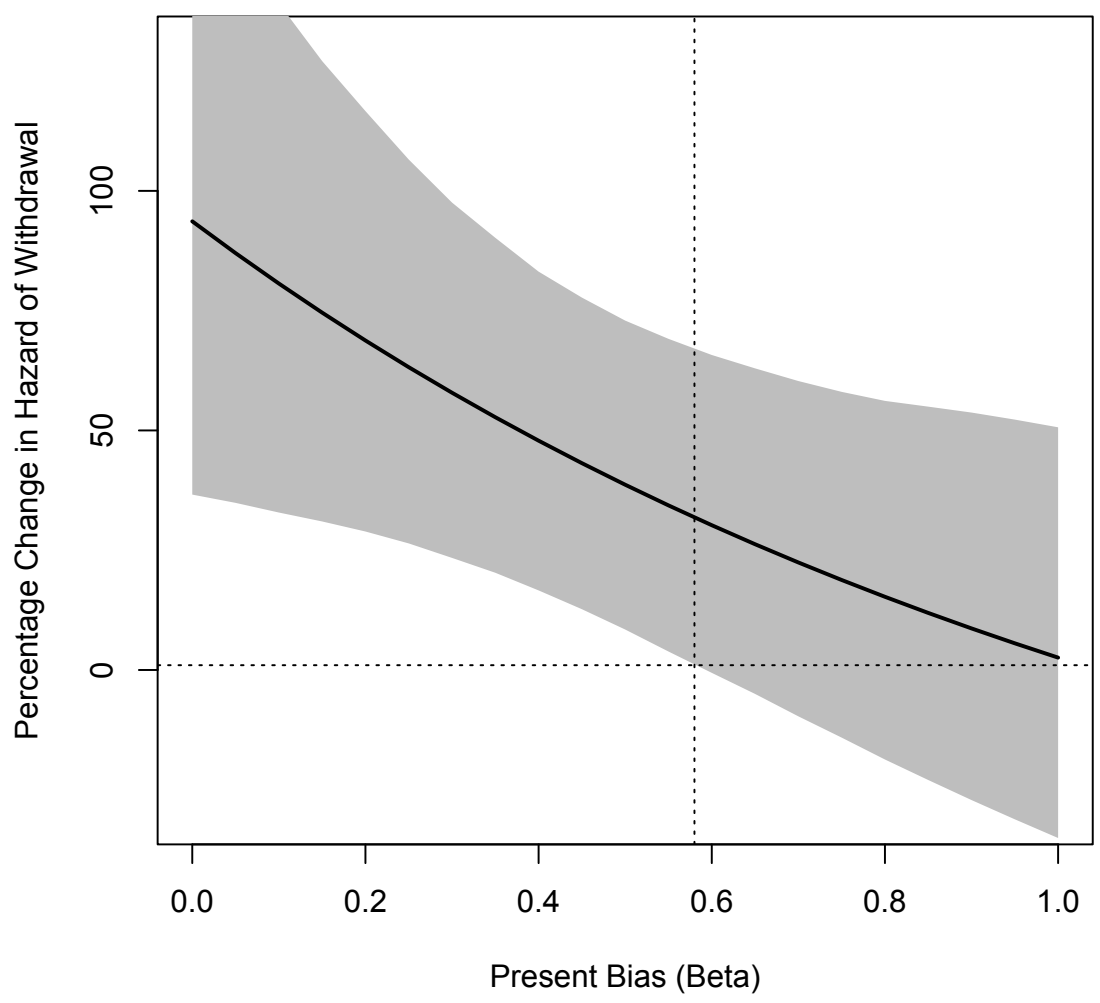

Individuals with high present bias (low $\beta$ ) are less resolute (more sensitive to casualties) than more patient individuals. The dark line indicates the point estimate of the combined coefficient for the conditional effect, while the shaded grey depicts $90 \%$ confidence bands calculated using draws from a multivariate normal.

In the dispositional analyses, $\beta$ parameters only approached statistical significance in the pro-intervention subsample, suggesting that present bias only affects resolve among participants who wanted to intervene in the first place. However, it displays significant interaction effects with the casualty manipulation in both the full sample ( $p<0.074)$ and pro-intervention subsample $(p<0.090)$, and in the direction predicted by the hypotheses: the more impatient participants are, the more 
the casualty manipulation makes them want to cut and run, while we fail to find evidence that relatively patient participants are significantly affected by the casualty manipulation. This interaction effect is illustrated in Figure 3.8, which plots the combined coefficient of the treatment effect conditional on participants' degree of present bias, with $90 \%$ confidence intervals, and the dashed vertical line indicating the point on the x-axis at which we can no longer distinguish the effect of the casualty treatment from zero. Notably, $\beta$ did not moderate the impact of the reputation cost manipulation in either the full or the pro-intervention subsample, suggesting that time preferences moderate the impact of the costs of fighting, but not the costs of backing down.

\section{Risk preferences}

As discussed above, the dispositional set of analyses showed that risk aversion was a highly significant predictor of participants' resolve, and that it displayed a quadratic effect, with resolve lowest amongst participants with moderate levels of risk aversion: as participants moved towards risk acceptance, they appeared to be more likely to want to stick things out, while as participants moved towards higher levels of risk aversion, they appeared to be more likely to maintain course. It was suggested that this curvilinear effect stemmed from the fact that both standing ground and cutting and running can be perceived as risky, depending on the circumstances. As shown by Figures 3.9-3.10, a series of interactions between risk aversion and the two main situational manipulations of interest - reputation costs, and casualties - offers support for this interpretation, and thus, support for $\mathrm{H}_{4} \mathrm{c}$.

First, risk aversion has a statistically significant interaction with the reputation manipulation in both the full sample $(p<0.075)$ and the pro-intervention subsample $(p<0.016)$, illustrated in Figure 3.9. The more risk averse participants are, the more persuaded they are by the reputation cost manipulation to stay the course. In contrast, we fail to reject the null hypothesis that the reputation cost treatment fails to affect resolve amongst those participants whose risk aversion scores are lower 
Figure 3.9: Risk preferences $x$ reputation cost combined coefficient plot

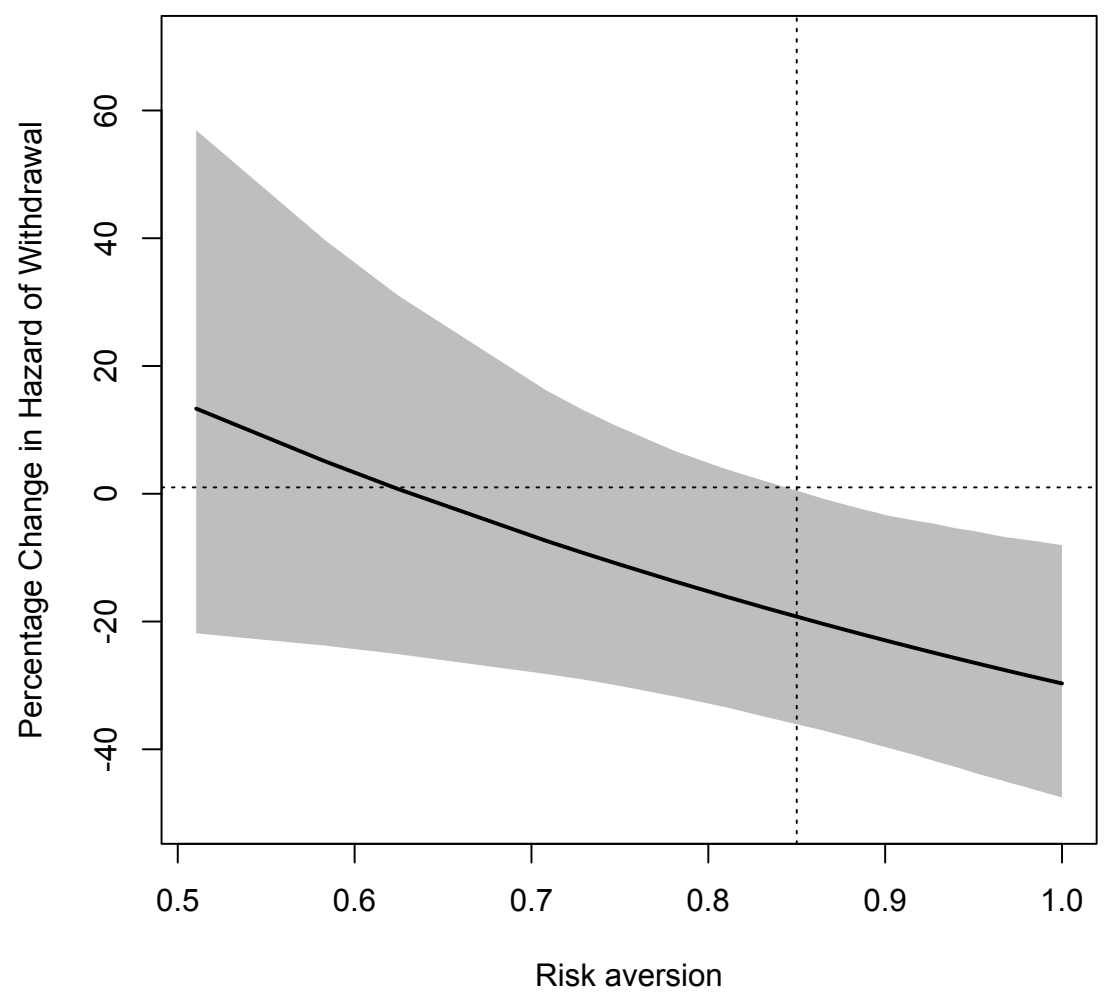

Individuals who are relatively risk averse are more sensitive to reputation costs. The dark line indicates the point estimate of the combined coefficient for the conditional effect, while the shaded grey depicts $90 \%$ confidence bands calculated using draws from a multivariate normal.

than $\rho=0.85$, suggesting that as risk acceptance increases, so to does willingness to risk the reputational costs that arise from backing down in the face of aggression; indeed, analyses on a manipulation check for perceived reputation costs finds a highly significant relationship $(p<0.014)$ : risk averse individuals are far more likely to view steep reputational costs for the US withdrawing, and the effect $(+0.56$ on a scale of 1-5) is as large as that of party ID.

Second, a similar interaction effect is evident with respect to casualties (although only in the pro-intervention subsample: $p<0.039$ ) but in the opposite direction. As Figure 3.10 shows, the more risk averse participants are, the more sensitive they are to the casualty manipulation, while we fail to find that casualties significantly 
Figure 3.10: Risk preferences $x$ casualty combined coefficient plot

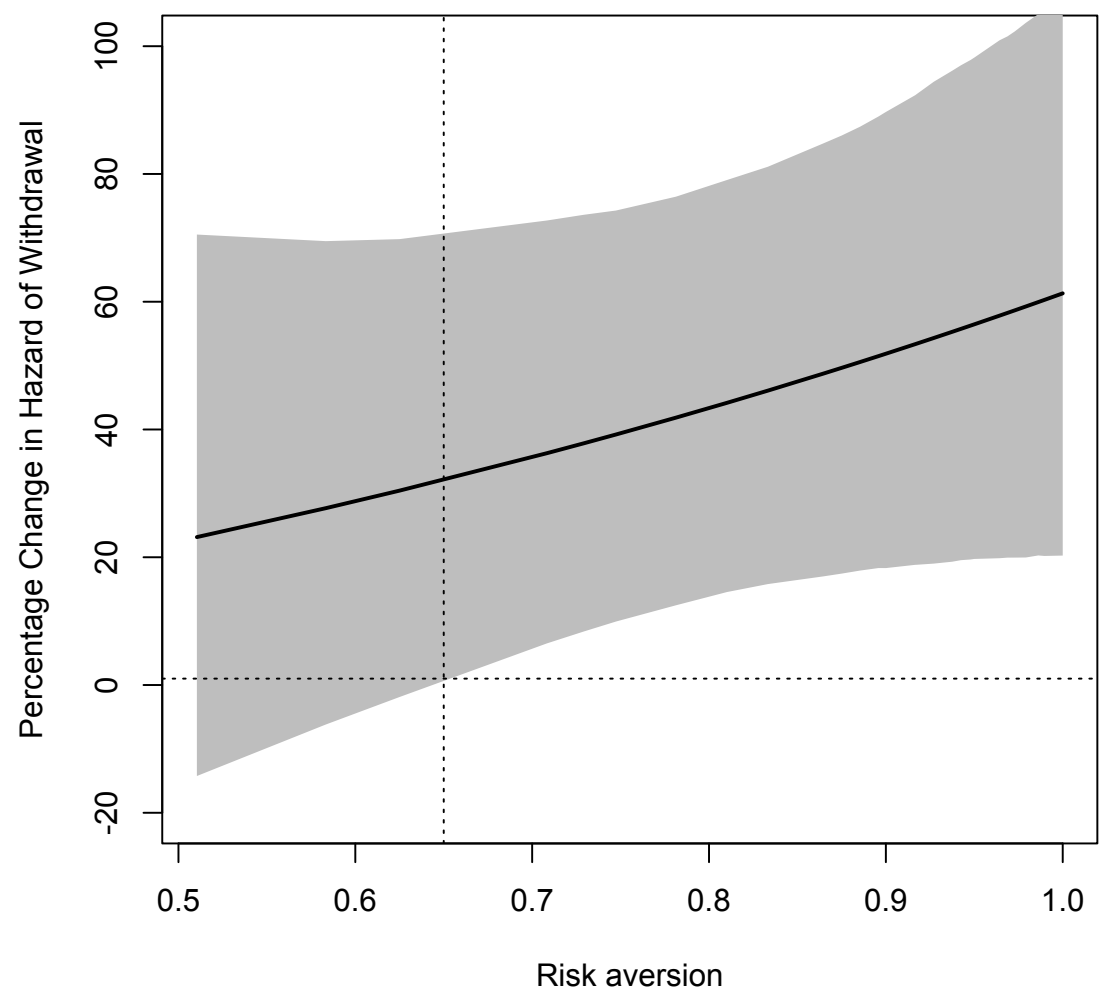

Individuals who are relatively risk averse are more sensitive to casualties. The dark line indicates the point estimate of the combined coefficient for the conditional effect, while the shaded grey depicts $90 \%$ confidence bands calculated using draws from a multivariate normal.

lower resolve for participants whose risk aversion scores are lower than $\rho=0.65$. Thus, for both manipulations, risk aversion's moderating effect is associated with greater caution, but in opposite directions, since concern about casualties lowers resolve while concern about reputation costs raises it.

\subsection{Conclusion}

Following his success leading the Eighth Army at the battle at El Alamein in 1942, Viscount Montgomery wrote that "the more fighting I see, the more I am convinced that the big thing in war is morale", and encouraged military scholars to "make a study of this subject." (Fennell, 2011, 1) Montgomery would therefore likely be 
pleased with contemporary research on international security, as resolve has become one of the most fundamentally important concepts in international politics, invoked by political scientists, pundits, and policy-makers alike. Despite this ubiquity, political scientists have done a poor job of explaining what exactly resolve is and where it comes from. In this chapter, I turned to experiments to test an interactionist theory of resolve in military interventions that uses specific dispositional characteristics to explain variations in sensitivity to particular situational features, and in so doing, disaggregates the cost of war.

The experimental results provide three main findings. First, although IR scholars have largely treated resolve as a situational feature, throughout the results the substantively largest effects are dispositional; the costs of war matter - when the costs of fighting increase, or the reputational costs of backing down are rendered less salient, actors are more likely to stay the course - but not as much as characteristics of actors themselves. Actors who are relatively impatient, moderately risk averse, low in cultures of honor, and scoring low in the perseverance subscale of trait self-control are also more likely to be irresolute. Predicting when actors will be more or less resolved thus demands paying closer attention to actor-level attributes rather than just the environment they face. However, although the importance of these dispositional factors in predicting resolve is significant, it is also important not to make too much of the imbalance in the relative contribution of situational and dispositional factors in the experiment. As I note in Chapters 4 and 5, it is possible that the situational treatments were relatively weak; after all the situational factors in the experiments are hypothetical, while participants' dispositional characteristics are real. Second, and relatedly, although the IR research on resolve has been largely disconnected from the growing body of research on willpower and self-control taking place outside political science, the major role played by the same sorts of dispositional factors that psychologists and behavioral economists routinely study when addressing similar questions in their own work suggests that resolve is not domain-specific, and that social scientists interested in resolve stand 
to gain by crossing disciplinary borders. Third, resolve is not only situational and dispositional, but also interactionist: impatient individuals are more sensitive to casualties than their patient counterparts, while risk averse individuals are more sensitive to both casualties and reputational costs.

These results raise a number of methodological issues for political scientists. First, this is the first experimental work in IR I am aware of that models both the selection into, and duration of, military operations. Although the international security literature tells us that war initiation and duration are two parts of the same continuous process (Filson and Werner, 2002), the extant experimental literature studies each step in isolation, modeling either the initial decision to use force (e.g. Herrmann, Tetlock, and Visser, 1999) or the question of whether troops should be withdrawn from a mission that has already begun (e.g. Boettcher and Cobb, 2006; Gartner, 2008b). Since the question of whether a mission is worth continuing is unlikely to be independent of the question of whether it was worth fighting in the first place (Gelpi, Feaver, and Reifler, 2009), modeling both phases in one experimental design allows us to explicitly model this selection process that IR scholars frequently discuss but rarely get to directly observe (Reed, 2000; Fearon, 2002; Clark, 2003; Sartori, 2005). Indeed, the results of the mediation analysis suggest that if one only analyzed the duration of an ongoing intervention, one would likely miss out on the importance of reputation costs. Second, the fact that the dispositional sources of resolve were measured in domains unrelated to that of the intervention scenario allows for greater confidence that the results are speaking to underlying dispositions, and in the case of risk attitudes, sidesteps the problem of selecting a meaningful reference point from which to judge what counts as risk acceptant or risk averse ( $\left.\mathrm{O}^{\prime} \mathrm{Neill}, 2001\right)$. The literature on risk and war frequently infers that leaders were in a state of risk acceptance based on their propensity to engage in conflict or make rash decisions (e.g. McDermott, 1998), implying that actors should be more belligerent the more risk acceptant they become. ${ }^{21}$ My results, however,

\footnotetext{
${ }^{21}$ The fact that much of this literature derives its predictions from prospect theory complicates the comparison, since prospect theory treats risk attitudes as situational (a function of whether par-
} 
show that when risk attitudes are measured independently of the behavior they are being used to explain, the relationship is highly nonlinear, and that moderately risk averse participants are likely to display more resolve than either their risk acceptant or highly risk averse counterparts.

Third, it is important to note that although much of the discussion of dispositional and situational theories of resolve have tended to treat rational choice theories of resolve as falling into the latter camp, there is nothing inherent in game theory that prevents rational choice scholars from emphasizing dispositional features. Indeed, both time and risk preferences - the two dispositional variables that display significant interaction effects with the situational manipulations - are integral parts of many rational choice theories of conflict. ${ }^{22}$ Largely for analytic reasons, however, much of the focus has been on actors' payoff structures, such that the discipline has been able to use "the costs of war" as shorthand for an actor's resolve. The above results, then, should less be seen as challenging game theoretic models of war, as more as reminding us that dispositional features merit at least as much attention as situational ones.

The findings also suggest a number of important implications for policymakers. The conventional image of the American public is of an audience highly sensitive to the costs of war (e.g. Mueller, 1971). The results here not only support Gelpi, Feaver, and Reifler (2009) in showing that different segments of the public respond to casualties in rather different ways, but remind us that war has costs beyond casualties, and that at least in the intervention scenario studied here, participants were also sensitive to the reputational costs of backing down. A White House interested in drumming up support for a military intervention it believes to be in the national interest may thus find widespread sympathy for reputational justifications for the intervention, although the results of the mediation analyses show

ticipants are in the domain of gains or the domain of losses) rather than as an actor's disposition (Kahneman and Tversky, 2000). I return to this point in Chapter 6.

${ }^{22}$ However, although repeated games model an actor's $\delta$ parameter, because they tend to assume exponential discounting functions, they do not incorporate a $\beta$ parameter, when the results here suggest that $\beta$, rather than $\delta$, displays the significant interaction effect with the cost of fighting. 
that these arguments will be most effective in raising the initial level of support for the mission, rather than prolonging support once the intervention has already begun. The significant effect of the anticipated cost manipulation in rendering participants more patient with a prolonged intervention suggests that one of the Bush administration's mistakes in selling the war in Iraq to the American people was in underselling the amount of force the mission would require, as displayed by Vice President Cheney's infamous suggestion in March 2003 that the US would "be greeted as liberators" by the Iraqi people. When President Bush posed with a "Mission Accomplished" banner on the deck of the USS Abraham Lincoln while announcing the end of major combat operations in Iraq in May 2003, he may have engendered a short-term boost in public support, but in the long run it made the turmoil that followed seem costlier in the public's eyes than it otherwise would have been.

Finally, the experiment also lays the groundwork for future research. First, although the use of undergraduate student samples is useful in gaining leverage over psychological processes (Druckman and Kam, 2011), it poses problems when trying to generalize the findings to the population at large. Thus, Chapter 4 presents the results from an embedded survey experiment administered to a national representative sample of adult Americans thanks to support from Time-Sharing Experiments for the Social Sciences (TESS). Second, since the purpose of the experimental analyses is to investigate the origins of resolve amongst ordinary members of the public, it deliberately sidesteps the levels of analysis question that, as noted in Chapter 2, would manifest itself for both the situational and dispositional variables if studied with observational data. Chapter 5 tackles this level of analysis question head-on. 


\section{Chapter 4: Experimental Microfoundations for Resolve: II}

The laboratory experiment results offer considerable insight into the situational, dispositional and interactionist determinants of resolve at the individual-level. Because of random assignment, the laboratory experiment offers causal validity for the situational variables, ${ }^{1}$ and in as much as the panel experimental structure corresponds to our posited data-generating process in which information about different types of costs of war is moderated by dispositional characteristics in shaping how much resolve actors display, offers construct validity as well (Shadish, Cook, and Campbell, 2002). However, there are at least two reasons why we would want to supplement these findings. The first concerns representativeness. The lab experiment is based on a student sample, and if we are interested in resolve in the context of American public opinion about military interventions, we might wish to run the experiment on a sample more representative of the American population as a whole. ${ }^{2}$ The student sample was younger, more educated, and disproportionately male compared with the American population as a whole; given changing societal expectations of war (Smith, 2005) and generational effects (Schuman, 1992), for example, it is possible that older respondents could respond to the casualty treatments in very different ways. The second concerns replication. Often

\footnotetext{
${ }^{1}$ The dispositional variables are, of course, not causally identified, since they are measured rather than manipulated.

${ }^{2}$ As Morton and Williams (2010, p. 265) argue, although political scientists frequently refer to representativeness as an external validity issue, external validity involves generalizing findings beyond the target population, such that we should think of it as an internal validity issue instead. For limitations on the inferences one can draw from a student sample, see Mintz, Redd, and Vedlitz (2006); for a contrary view, see Druckman and Kam (2011). Of course, even a national representative American sample will not be representative of humanity as a whole - see Henrich, Heine, and Norenzayan (2010).
} 
times in survey research the conclusions we draw are influenced by the instrumentation we employ (Presser and Schuman, 1981). If we find similar results despite using slightly different operationalizations of some of the key concepts, it offers greater faith in our findings, and further confidence in our understanding of the microfoundations of resolve.

Thus, in the summer of 2012, an online survey experiment was administered to a national probability sample of 1078 adults, replicating parts of the lab experiment while also seeking to generalize the findings to the public writ large. ${ }^{3}$ As a national probability sample, the survey participants look like the American public as a whole, and compared to the lab experiment, are older (on average, survey participants were 49.4 years old, compared to 21.3 in the lab experiment), less educated (38.7\% received a high school education or less), and feature slightly lower proportions of Caucasians ( $74.4 \%$ of survey respondents, compared to $77.4 \%$ of lab experiment participants), and males (50.0\% of respondents identified as male in the survey experiment, compared to $60.1 \%$ in the lab experiment).

\subsection{Materials}

\section{INTERVENTION SCENARIO}

Similar to the laboratory experiment, the survey consisted of three parts: a factorial experiment manipulating the human and reputational costs of a hypothetical military intervention, a dispositional questionnaire measuring time preferences, risk preferences, and honor orientations, and a concluding questionnaire measuring general demographic characteristics. As was the case with the lab experiment, participants randomly received either the dispositional questionnaire, or the inter-

\footnotetext{
${ }^{3}$ The study was fielded by GfK Custom Research (formerly known as Knowledge Networks), thanks to support from the National Science Foundation's Time Sharing Experiments for the Social Sciences initiative. 2051 panelists were randomly drawn from the GfK panel; 1263 responded to the invitation, 1078 of whom qualified for the survey, producing a field stage completion rate of $61.5 \%$ and a qualification rate of $85.4 \%$. The recruitment rate was $15.2 \%$ and the profile rate was $65.4 \%$, for a cumulative response rate of $6.1 \%$. The survey was administered online, and the median survey length was 7 minutes.
} 
vention scenario, first, such that the experimental design is once again a 2 (ex ante human costs: low or high) x 2 (ex post human costs: low or high) x 2 (reputation costs: implicit or salient) x 2 (order manipulation: scenario 1st or 2 nd) fully-crossed factorial experimental design. The structure of the experiment is summarized in Figure 4.1, below.

In the intervention scenario, as before, participants are presented with a hypothetical military intervention on behalf of an ally under siege, and asked whether they think the United States should send troops; regardless of participants' responses, the intervention proceeds, and participants are then asked about the possibility of withdrawing troops. The intervention scenario differs from its predecessor in two ways. First is in the measurement of the dependent variable. Whereas the laboratory experiment followed Gartner (2008a) in imposing a panel structure, in which participants receive information about the intervention once a "year" and are presented with the option of withdrawing after each briefing (for up to seven years), cost constraints mean that the survey experiment relies on a simple one-shot measurement technique, in which, after the first year, participants are asked how long they think troops should remain involved in the mission.

Second, compared to the lab experiment, the survey experiment features steeper reputation costs. In the lab experiment results, the reputation cost treatment raised resolve by around $18 \%$, but the treatment effect was only significant at around the $p<0.11$ level, and the manipulation check was not significant $(p<0.324)$, although this may also have been because it was administered after the intervention scenario had already ended, such that motivated reasoning may have led participants who advocated withdrawal to respectively underweight factors that lead to a different decision. Thus, the survey experiment sought to raise the reputational costs of backing down by rendering them more salient, while also relocating the manipulation check to immediately after the treatment was presented, before participants had the chance to make any decisions. In the new formulation of the reputation cost treatment, an ally the participant cares a lot about publicly warns that if the 


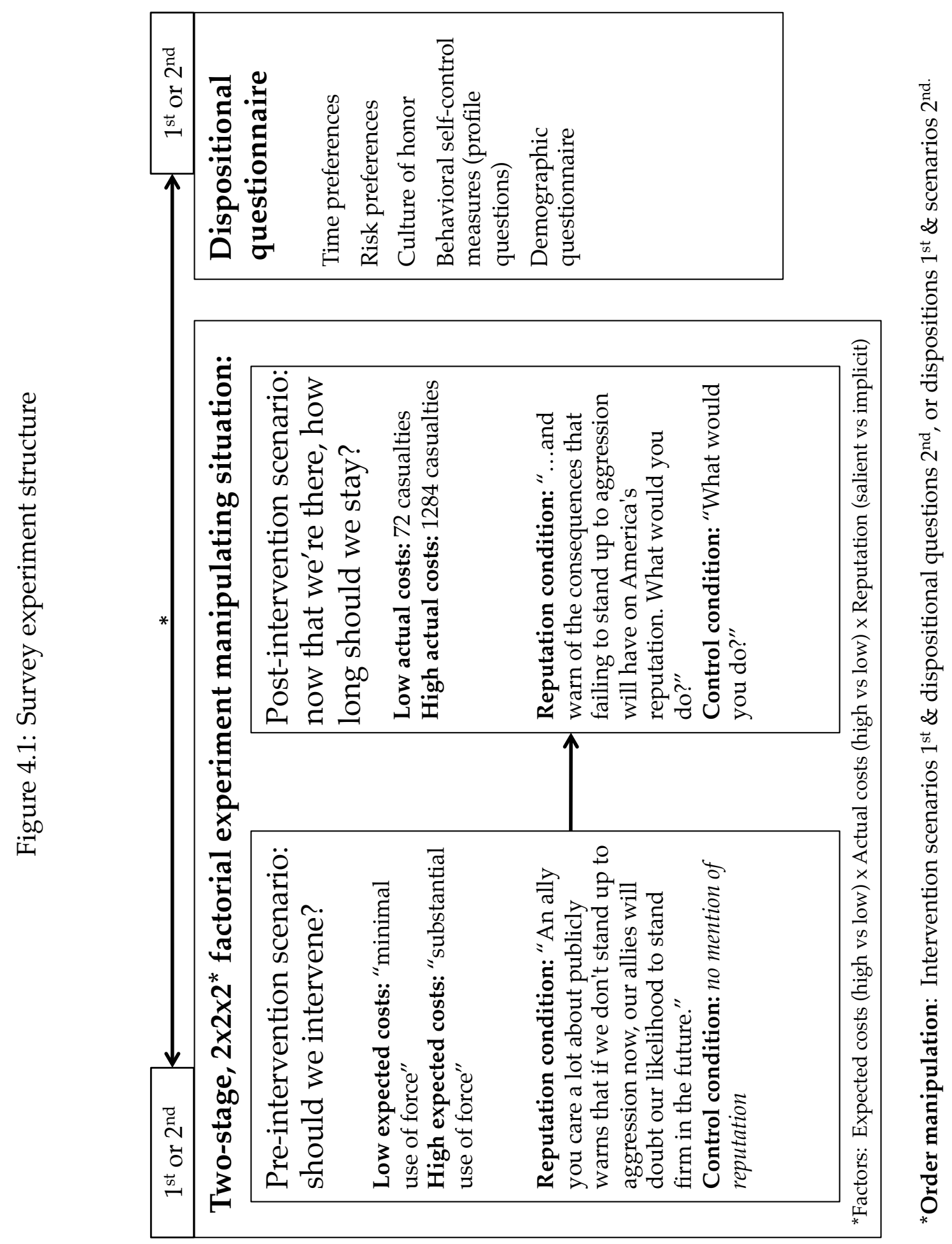


United States does not stand up to aggression now, our allies will doubt our likelihood to stand firm in the future. The element of publicity is important here, since reputation costs require an audience capable of monitoring behavior (Schelling, 1960, 29-30, Fearon, 1994; Krebs and Jackson, 2007), so the treatment should render the reputational consequences of backing down more salient. As before, participants are presented with a manipulation check asking about potential damage to the US reputation, but in the survey experiment, the manipulation check immediately follows the pre-intervention scenario, rather than after the intervention has concluded, and thus refers to the reputational consequences of the U.S. not intervening, rather than the reputational consequences of the U.S. withdrawing from the intervention.

\section{DisPositional QUESTIONNAIRE}

The dispositional questionnaire of the survey experiment measures a number of dispositional characteristics, which partially overlap with those featured in the lab experiment, but also include a number of extensions. Time preferences are measured by participants' $\delta$ parameter, which corresponds to how much the participant values the future relative to the present. As in the lab experiment, $\delta$ is estimated using the values respondents provide to the two matching questions (with a one-year and ten-year delay, respectively):

$$
\delta=\left(\frac{X_{1}}{X_{10}}\right)^{1 / 9}
$$

Risk attitudes are measured in three different ways. First, as in the lab experiment, lottery tasks are used to estimate participants' degree of risk aversion (as measured by $\rho$, the relative risk premium); higher values of $\rho$ indicate higher degrees of risk aversion. As before, $\rho$ is estimated by averaging across the risk premia calculated based on the responses participants provide to the two lottery questions: 


$$
\begin{array}{r}
R_{1}=\frac{X}{100}-1 \\
R_{10}=\left(\frac{X}{100}\right)^{0.1}-1
\end{array}
$$

However, prospect theory notes that risk attitudes vary depending on whether individuals are in a domain of gains of losses (Kahneman and Tversky, 2000; for applications to International Relations, see Boettcher, 1995; Berejekian, 1997; Levy, 1997; McDermott, 1998). Whereas the previous lottery questions measured risk aversion, or risk attitudes in the domain of gains, a second set of lottery questions were used to measure loss aversion, or risk attitudes in the domain of losses. Participants were presented with two lottery questions in which they had a (i) 50\% chance to lose $\$ 25$ but a $50 \%$ chance to win $\$ X$, or (ii) a $50 \%$ chance to lose $\$ 100$ but a $50 \%$ chance to win $\$ X$, and asked how much $X$ would have to be to make them want to buy a ticket. Loss aversion $(\theta)$ parameters were estimated for each question by dividing each $\$ \mathrm{X}$ by 2 , and then averaging across the two $\theta$ s to produce an average measure of loss aversion.

$$
\theta=\frac{\frac{X_{1}}{2}+\frac{X_{2}}{2}}{2}
$$

Relatedly, in the psychological literature, risk attitudes are frequently studied as a general dispositional orientation measured with self-report items (e.g. MacCrimmon, Wehrung, and Stanbury, 1986; Kowert and Hermann, 1997; Kam and Simas, 2010). Accordingly, a dispositional measure of risk orientation ("In general, people often have to take risks when making financial, career, or other life decisions. Overall, how would you place yourself on the following scale?", with a seven-point scale with options ranging from "Extremely comfortable taking risks" to "Extremely uncomfortable taking risks") was borrowed from Ehrlich and Maestas (2010), reverse-coded such that higher values indicate greater degrees of risk acceptance. 
Third are honor orientations. The survey experimentation measures honor orientation differently from the lab experiment, employing items from a scale developed in cross-cultural psychology by Osterman and Brown (2011). Although the honor measure in the lab experiment was indeed associated with higher levels of resolve, its reliability was relatively low, its effects were no longer significant once political ideology was controlled for, and it lacked the anticipated interaction effect with the reputation cost treatment. The new three-item scale ("A real man never leaves a score unsettled," "A real man is seen as tough in the eyes of his peers," and "A real man should be willing to defend himself if he's insulted in public") taps into similar constructs as the previous measure in its emphasis on standing up for oneself, reputations for toughness, and so on, but also has the advantage of being developed and validated outside the realm of this study. ${ }^{4}$

Fourth is trait self-control. The lab experiment included the perseverance and urgency subscales of Whiteside and Lynam's (2001) trait self-control scale — which measures trait self-control using cognitive self-report items (e.g. “Unfinished tasks bother me"), but social scientists often measure self-control using either behavioral tasks (Baumeister, Vohs, and Tice, 2007; Galliot and Baumeister, 2007), or behavioral report items (Tittle, Ward, and Grasmick, 2003). Thus, I also include items measuring the frequency with which participants report exercising, "avoiding food high in fat and/or calories," and smoking - since self-control understood to be associated with success in pursuing these first two activities, and successful avoidance of the latter (Perri, Richards, and Schultheis, 1977; Sniehotta, Scholz, and Schwarzer, 2005; Papies, Stroebe, and Aarts, 2008). Although these items are relatively far removed from the world of international politics, if the predictors of political will are indeed related to the predictors of personal will, we might imagine that participants who score high in the behavioral trait self-control measures

\footnotetext{
${ }^{4}$ The Osterman and Brown honor measure employs more gendered language than the honor scale in previous chapter, but the reliability of the scale does not significantly vary based on the gender of the participant ( $\alpha=0.78$ for males, $\alpha=0.75$ for females), and when interaction terms between honor and gender are added to Table 4.2, they are never significant $(p<0.405$ and $p<$ 0.303 , for models 1 and 2 , respectively.)
} 
will also display greater persistence in the military intervention scenario. Principal axis factoring with oblimin rotation suggests the three items load onto a single factor, although inspection of the factor matrix suggests that smoking habits load less strongly than exercise and dietary habits, so regression factor scoring was used to create a measure of behavioral self-control, rescaled so the factor scores range between 0 and 1 for ease of interpretability.

\subsection{Results}

As in the previous chapter, the results are discussed in a number of phases, first examining the dependent variable of interest; second, analyzing the impact of the situational treatments; third, incorporating the dispositional characteristics into the analysis, and fourth, testing the interactionist hypotheses. Finally, the conclusion compares the survey experiment results with that of the lab experiment, and offers some broader comments.

\section{HOW RESOLVED WERE THE PARTICIPANTS?}

Figure 4.2 shows that as was the case in the lab experiment, there was considerable variation in participants' resolve. On the whole, the national sample was less inclined to intervene than the lab experiment participants, as only $53 \%$ of survey respondents supported the United States sending troops, compared to nearly $70 \%$ support in the student sample. Given that the survey experiment featured a steeper

reputation cost manipulation - which likely boosted participants' initial degree of support for intervening compared to the setup in the lab experiment - the fact that support for the intervention actually dropped by 17 percentage points shows just how much less interventionist the national sample was compared to the student sample. That difference aside, however, the general pattern in the distribution of responses is the same. First, participants display a clear variation in resolve, with $16.0 \%$ of respondents wanting to pull out troops after the first year, and $19.9 \%$ of 
Figure 4.2: Initial attitudes towards the mission predict the duration of support

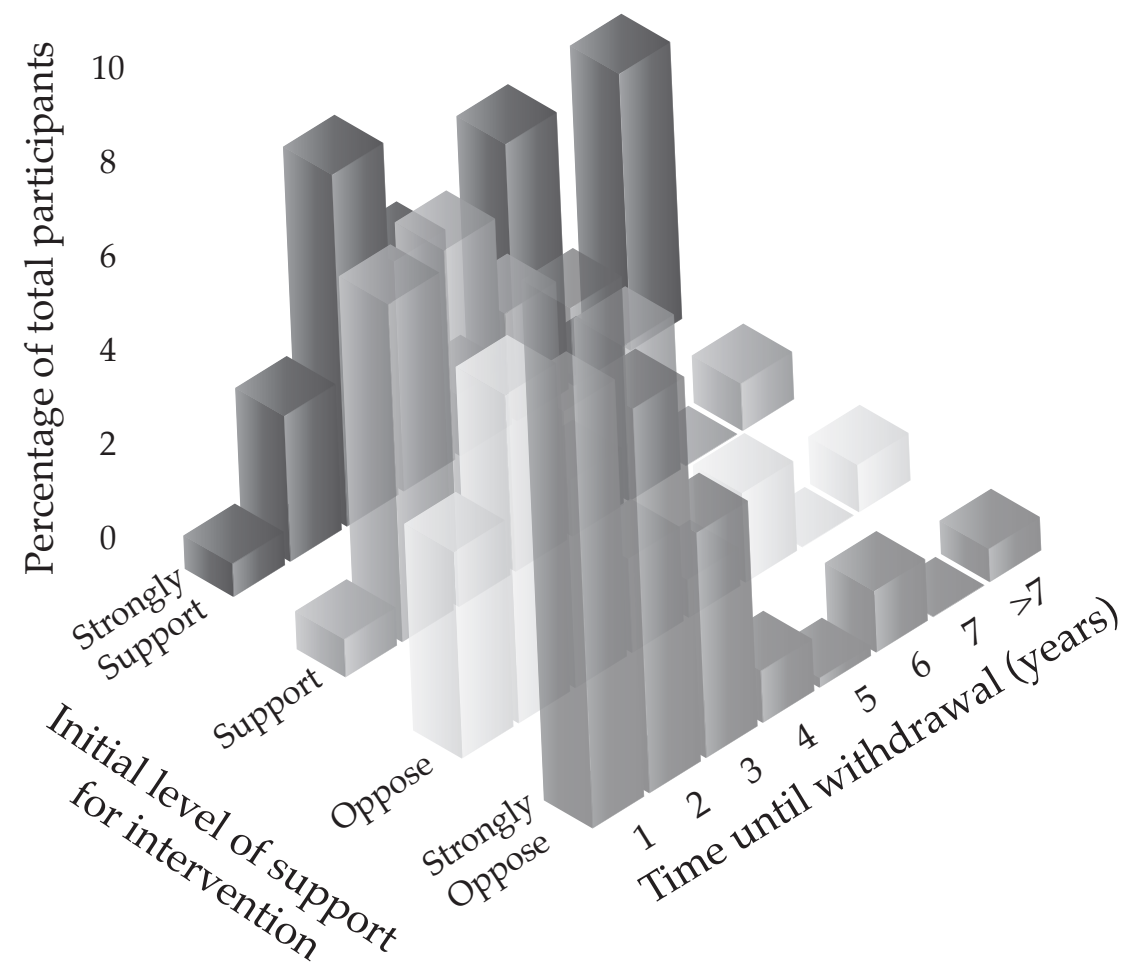

respondents calling for troops to remain six years or longer. Second, the amount of time participants wanted troops to stay depended on whether they wanted troops to be there in the first place $\left(\chi^{2}=358.81, p<0.000\right): 40.5 \%$ of respondents who strongly opposed the US sending troops advocated for withdrawal after just one year, compared to only $2.4 \%$ of the respondents who strongly supported the intervention. Thus, as was the case in the lab experiment, I find little evidence of sunk cost logic driving attitudes towards the intervention, as participants who initially opposed intervening were unlikely to change their mind once troops were actually deployed; "sunk cost frames" may indeed mobilize support in between-subject public opinion experiments (Boettcher and Cobb, 2009), but the two-stage withinsubject setup of the experimental designs employed here show no indication that participants who were initially opposed to the mission become more supportive once boots hit the ground. 


\section{Situational TREatments}

Table 4.1 and Figure 4.3 depict the treatment effects for the four treatments (anticipated costs, reputation costs, casualties, and the order manipulation). ${ }^{5}$

Table 4.1: Treatment effects based on initial decision to invade

\begin{tabular}{lcc|cc|cc}
\hline & \multicolumn{2}{c}{ All participants } & \multicolumn{2}{c}{ Go in } & \multicolumn{2}{c}{ Stay out } \\
\hline & $\beta$ & Hazard Ratio & $\beta$ & Hazard Ratio & $\beta$ & Hazard Ratio \\
\hline Anticipated costs & -0.079 & $-7.6 \%$ & -0.130 & $-12.2 \%$ & -0.145 & $-13.5 \%$ \\
& $(0.062)$ & {$[-16.6 \%, 2.3 \%]$} & $(0.087)$ & {$[-23.9 \%, 1.2 \%]$} & $(0.092)$ & {$[-25.6 \%, 0.6 \%]$} \\
Reputation costs & -0.130 & $-12.2 \%$ & -0.131 & $-12.3 \%$ & -0.224 & $-20.0 \%$ \\
& $(0.062)$ & {$[-20.8 \%,-2.8 \%]$} & $(0.086)$ & {$[-23.9 \%, 1.1 \%]$} & $(0.091)$ & {$[-31.2 \%,-7.1 \%]$} \\
Casualties & -0.071 & $-6.8 \%$ & -0.090 & $-8.6 \%$ & -0.066 & $-6.4 \%$ \\
& $(0.062)$ & {$[-15.9 \%, 3.2 \%]$} & $(0.087)$ & {$[-20.8 \%, 5.4 \%]$} & $(0.092)$ & {$[-19.5 \%, 8.8 \%]$} \\
Order & -0.694 & $-6.7 \%$ & -0.013 & $-1.3 \%$ & -0.205 & $-18.5 \%$ \\
& $(0.062)$ & {$[-15.8 \%, 3.3 \%]$} & $(0.087)$ & {$[-14.4 \%, 13.8 \%]$} & $(0.091)$ & {$[-29.8 \%,-5.3 \%]$} \\
$\mathrm{N}$ & & 1039 & & 540 & & 498
\end{tabular}

Main entries are Cox model coefficients; SEs in parentheses; 90\% CIs around hazard ratios in brackets. Positive coefficients indicate a greater likelihood of 'cutting and running.'

As was the case in the lab experiment results, the first model of the table depicts the treatment effects for the full sample; the second model looks at the treatment effects for those respondents who initially supported the intervention, while the final model displays the treatment effects solely amongst those respondents who never supported intervening in the first place. ${ }^{6}$ These same effects are visually depicted in Figure 4.3, which also plots the percentage change in the hazard of withdrawal from the full sample with population weights added. ${ }^{7}$

\footnotetext{
${ }^{5}$ Because the dependent variable was measured with an open-ended question, there are extreme outliers in the right tail of the distribution of the dependent variable; $90 \%$ of respondents advocate withdrawal after six years, but $2.5 \%$ of respondents were still willing to let the intervention persist after 11 years (and one respondent suggested 1001 years). Following an inspection of the score residuals, I d ropped these 27 outliers from the analysis, since residual-based tests of the proportional hazard assumption are sensitive to other forms of model misspecification (Keele, 2010). Note that because all participants experienced failure (in an event history sense, rather than a Freudian one), the $\mathrm{N}$ listed in Table 4.1 refers to both the number of participants and the number of events.

${ }^{6}$ Thus, Table 4.1 differs from its counterpart in the previous chapter in that the larger sample size allows us to compare the determinants of resolve between the pro- and anti-intervention subsamples, rather than just comparing the pro-intervention subsample with the full sample.

${ }^{7}$ The population weights post-stratify on a variety of demographic characteristics to adjust for non-response and over- or -under-sampling of particular groups, such that the data speak to the
} 
Figure 4.3: Coefficient plot of situational determinants of resolve

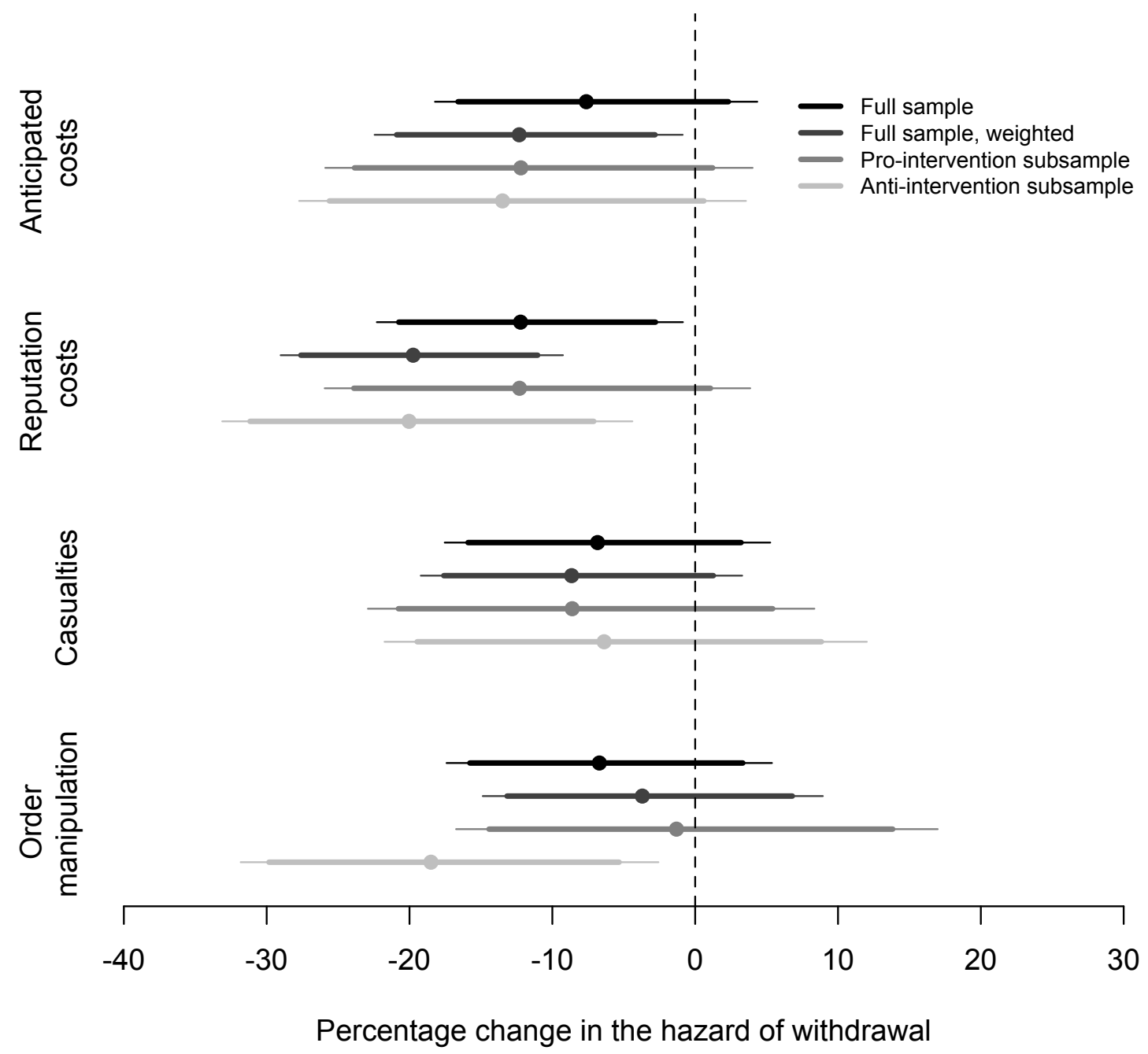

Point estimates of percentage change in the hazard ratio incurred by each of the four experimental manipulations, with 90 and $95 \%$ confidence intervals. Negative values indicate a decreased probability of withdrawal, and thus, greater resolve. 
The treatment effects plotted in Figure 4.3 suggest three important points. First, the reputation cost treatment displays the same resolve-inducing effect as in the lab experiment, but at a higher degree of statistical significance: participants who received the reputation cost treatment were $12.2 \%$ less likely to withdraw at any given time (a rate that grows to $19.7 \%$ once the population weights are added, and $20.0 \%$ amongst the anti-intervention subsample). The only model where the reputation cost treatment failed to achieve statistical significance was amongst the prointervention subsample, although the nonparametric mediation analyses reported in Figure 4.4 below find that the impact of reputation costs is not mediated by the initial decision to intervene. In the lab experiment, the reputation cost treatment displayed a "selection effect," in that participants took reputation costs into account when deciding whether to intervene initially, which thereby had no effect once the intervention took place. The shaming ally reputation cost treatment in the survey experiment, however, operates through a different causal pathway: the warnings from the ally did not affect the initial decision to intervene, but did make participants more likely to want to stay once the intervention took place. ${ }^{8}$

Second, while the costs of backing down play a larger role in the survey expopulation of American adults as a whole. As Mutz (2011, chapter 7) notes, practices differ in the survey experiment literature as to the handling of population weights; scholars who approach survey experiments from a survey tradition tend to employ weights regularly because they enable the researcher to extrapolate to the population as a whole, whereas scholars who approach survey experiments from the experimental tradition tend to be less enamored with weights, since the estimates of the sample treatment effects are already unbiased without them. Here, I restrict the use of weights to one of the models presented in Figure 4.3, showing how the treatment effects change when weights are added, but do not include weights in any of the dispositional analyses that follow, for two reasons. First, many of the dispositional variables are likely to be correlated with the demographic characteristics used to predict selection into the sample - and thus, weighting can cause us to underestimate the effect of the dispositional variables (Brehm, 1993, p. 119). Second, and relatedly, the subsequent analyses also control for many of the demographic characteristics that are themselves being used to generate the weights. For more on the difficulties that arise with the use of survey weights in a multivariate context, see Winship and Radbill (1994); Gelman (2007). I am grateful to Chris Skovron for helpful conversations on this matter.

${ }^{8}$ As was the case in the previous chapter, the nonparametric mediation analyses were conducted with mediation version 4.0 (Tingley et al., 2012), which estimates the survival model using parametric Weibull models rather than the semiparametric Cox models employed in the main analyses. The pattern of results are similar to those presented in Table 4.1, although the casualty and anticipated cost treatments more closely approach statistical significance in a Weibull model than in a Cox model. 
periment results than in the lab experiment, the costs of fighting play a somewhat different one. Casualties appear to raise rather than lower participants' resolve, although the treatment fails to achieve statistical significance across all four models - a pattern explored in detail in the next section. Similarly, although the anticipated cost treatment is statistically significant once population weights are added (participants warned in advance of high expected costs of fighting were 19.7\% less likely to withdraw at any given point in time), it otherwise fails to achieve statistical significance. Many of these rejections of the null hypothesis are relatively narrow, but it is notable that our uncertainty around the treatment effect would be greater in the survey experiment than its lab experiment counterpart, given that the sample size is three times bigger in the former than the latter. Results from nonparametric mediation analyses depicted in Figure 4.4 offer one potential explanation for the weak effect of the anticipated cost treatment: higher expected costs of fighting make participants both less likely to want to intervene, and more patient once the intervention takes place, an "inconsistent mediation effect" in which the average causal mediation effect (ACME) and direct effect point in opposite directions, thereby suppressing the magnitude of the total effect (MacKinnon, Krull, and Lockwood, 2000).

Finally, although the order manipulation achieves statistical significance for the anti-intervention subsample (in which participants who completed the dispositional questionnaire prior to the intervention scenario were $18.5 \%$ less likely to withdraw at any given point in time), the manipulation on the whole remains nonsignificant, suggesting that completing the dispositional questionnaire does not significantly affect participants' responses to the intervention scenario, increasing our confidence in the results. ${ }^{9}$

\footnotetext{
${ }^{9}$ Similarly, a series of Wilcoxon rank-sum tests suggest that responses to the dispositional questionnaire are not significantly affected by the intervention scenario. Of the items in the dispositional battery, only the responses to the lottery measures of risk aversion significantly change as a result of the order manipulation ( $W=143022.5, p<0.031$ ), and the difference is both substantively small (the medians of each category differ by 0.008 for a variable whose values range from -0.875 to 1 ), and disappears when the results are analyzed using a t-test rather than a rank-sum test $(t=0.338$, $p<0.74)$.
} 
Figure 4.4: Nonparametric mediation effects: survey experiment
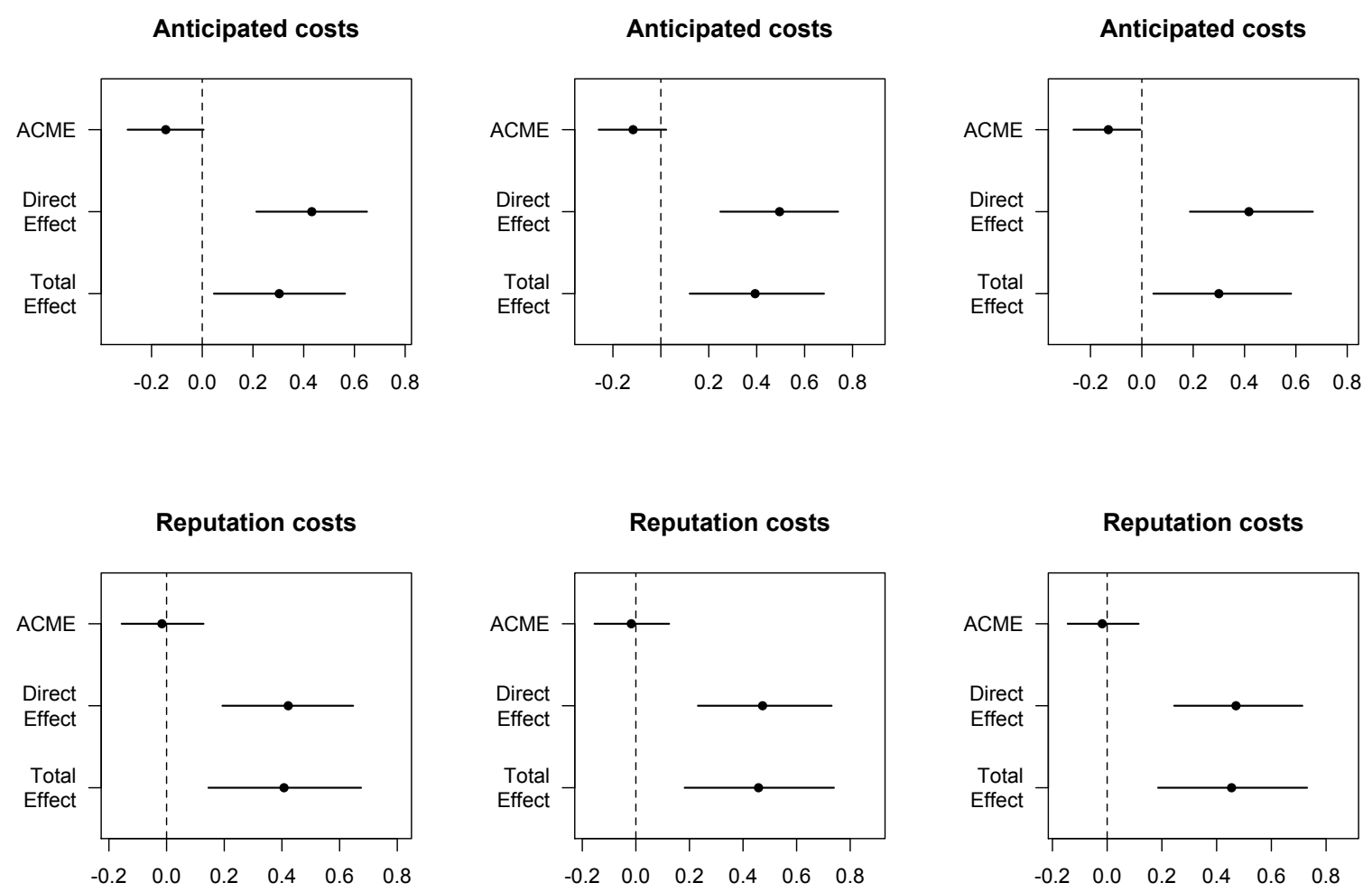

(a) No pretreatment covariates

(b) Dispositional controls

(c) Dispositional \& demographic controls

Mediation effects plotted using the mediation package from Tingley et al. (2012). All models are estimated using 1500 simulations with $90 \%$ quasi-Bayesian confidence intervals. Anticipated costs bolster resolve by making participants more patient after the intervention takes place, while reputation costs bolster resolve by making participants more likely to approve of the mission in the first place. Because the software cannot currently conduct sensitivity analyses on duration data, I run three iterations of the analyses: first, on a model with no pretreatment covariates, second, controlling for the dispositional factors of interest, and third, controlling for both the dispositional variables and demographic characteristics. I thus cannot eliminate the possibility that the results are an artifact of pre-treatment confounders, but I can test how robust they are to the inclusion of a large number of pre-treatment controls. 


\section{WHY DO CASUALTIES FAIL TO AFFECT PARTICIPANTS' RESOLVE?}

There are at least four potential classes of explanations for the divergent roles of casualties across the lab and survey experiment. First, the manipulations in the survey experiment could have failed. The manipulation check for the casualty treatment rules out this possibility: a Wilcoxon rank-sum test confirms that participants in the high-casualty condition predicted the US would suffer greater casualties the following year than participants in the low-casualty condition $(W=52829$, $p<0.000)$. Relatedly, if the casualty treatment failed, we would use the casualty manipulation check to identify those participants who estimated casualty levels closer to those specified in the manipulation, and estimate the treatment effect for just this subset of "compliers." However, as explained in detail in Appendix C.1, the casualty treatment consistently lacks a significant effect regardless of how compliance is operationalized.

Second, the casualty treatment's effects could be swamped by the steeper reputation cost treatment: 1284 casualties a year might not seem particular costly given the public rebuke by the close ally. If this were the case, however, we would see a statistically significant interaction effect between the casualty and reputation treatments, since the casualty treatment effect would be suppressed for those participants exposed to the reputation treatment, but not the reputation control. However, the lack of a statistically significant interaction effect between the two treatments $(p<0.826)$ suggests this not to be the case.

Third, the lack of a significant casualty treatment could be the result of treatment heterogeneity (Green and Kern, 2012): if the treatment effect varies with subgroups of the population, a nonsignificant average treatment effect would obscure significant conditional average treatment effects - hence offering a potential explanation for why treatment effects that were significant in a lab experiment on a relatively homogenous sample should lose their significance on a relatively heterogenous one. ${ }^{10}$ For example, respondents raised in the era of the Second World War might

\footnotetext{
${ }^{10}$ Indeed, Druckman and Kam (2011) note that if a treatment effect is homogenous, results from
} 
maintain different "analogies of war" (Khong, 1992) than those who came of age in the war on terror, such that the high casualty treatment might lack sufficient "dosage" for older respondents. ${ }^{11}$ Similarly, since college-age participants would be more likely to shoulder the costs of fighting (Horowitz and Levendusky, 2011), it is possible that their responses to the casualty treatment would differ from those of older generations. However, as the results in Tables C.1 - C.5 in Appendix C show, the impact of the casualty treatment is not conditional upon age, education, geographic region, income, or gender; the only treatment heterogeneity stemming from demographic characteristics occurs with respect to ethnicity, in that Hispanics are 38\% less likely to withdraw than non-Hispanic whites when they receive the high-casualty treatment - but this difference is not large enough to be driving the results.

Since the casualty treatment's effect seems not to stem from manipulation failures, a stronger reputation treatment, or treatment heterogeneity, the most likely explanation stems from the measurement of the dependent variable itself. The lab experiment utilizes a panel structure that presents the casualty information multiple times until the scenario ends or the participants withdraw, whereas the survey experiment uses a one-shot design in which participants only receive casualty information one year into the intervention, which offers little sense of whether the casualties are temporary or part of a broader trend, and instead forces them to extrapolate based on relatively little information (see Gartner, 2008a).

Figure 4.5 below uses the results of the casualty manipulation check to plot the distributions of the bootstrapped mean casualty predictions for both the lab and survey experiments, at each level of the treatment; since the lab experiment utilizes a panel structure, the mean casualty predictions are plotted across each wave of the study as well as by casualty treatment condition:

a convenience sample should generalize easily to a representative one.

${ }^{11}$ In as much as these analogies of war are shaped by previous personal experiences, we can consider them to be "pretreatment effects." (Gaines, Kuklinski, and Quirk, 2007; Druckman and Leeper, 2012) 


$$
\widehat{y}_{i t}=\frac{1}{B-1} \sum_{b=1}^{B} \bar{x}_{i t}{ }^{*}
$$

Since the survey experiment follows a one-shot design, it only has one wave, so the casualty predictions are plotted solely by treatment condition:

$$
\widehat{y}_{i}=\frac{1}{B-1} \sum_{b=1}^{B} \bar{x}_{i}{ }^{*}
$$

Figure 4.5: Bootstrapped mean casualty predictions by experiment format

Lab experiment

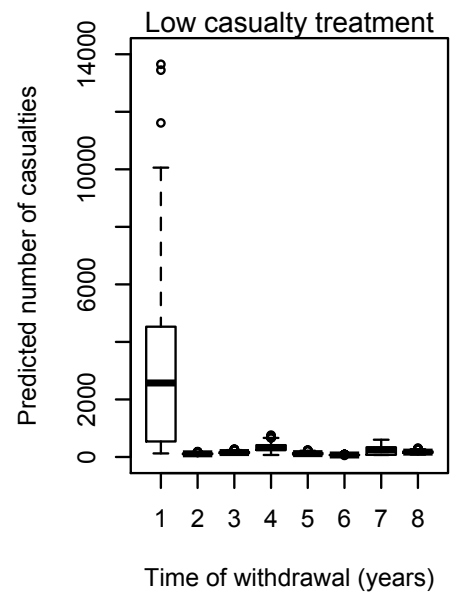

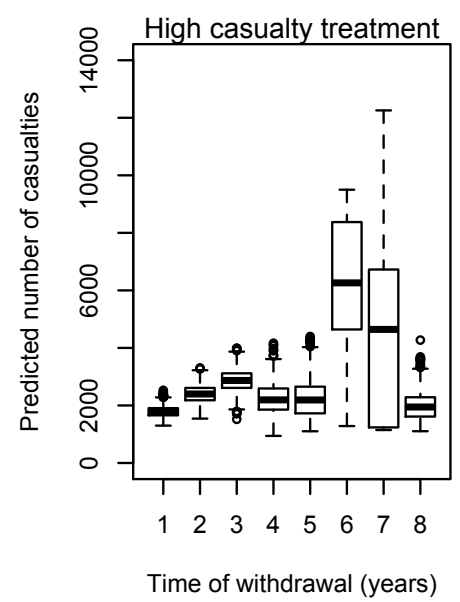

Survey experiment

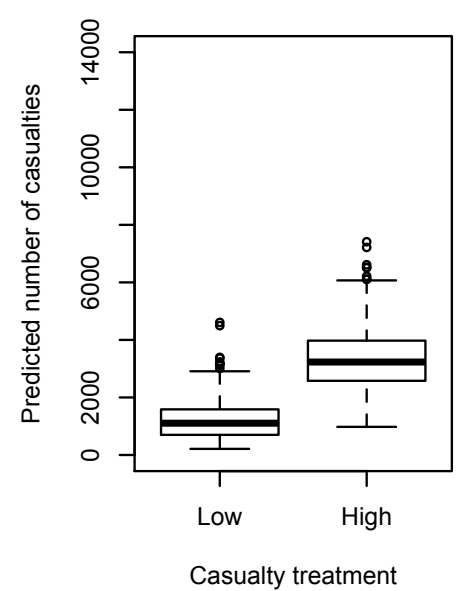

The bootstrapped mean responses $(B=1500)$ to the casualty manipulation checks across each wave of the lab and survey experiment by treatment group show that differences in experiment format may be responsible for the divergent effects of the casualty treatment between the lab and survey experiment: in a one-shot format, participants' uncertainty about casualty trends is relatively high, especially in the high-casualty condition. When the lab experiment results are pooled, bootstrapped calculations indicate that although there is no significant difference between the variance of manipulation check results for the low-casualty treatment in the lab and survey experiment $(p<0.24)$, the variance of the manipulation check results for the high-casualty treatment is significantly greater in the survey experiment than the lab experiment $(p<0.00)$.

The boxplots show that the casualty manipulation checks work - that is, that participants predict higher levels of casualties in the high casualty treatment than 
in the low casualty condition - but more importantly for our purposes, also show changes in the variance of the mean casualty predictions at the time participants withdraw, providing a sense of their uncertainty about casualty trends. ${ }^{12}$ The variance in the survey experiment is relatively high, despite the far larger sample size, whereas the variance in the lab experiment settles down in the low casualty treatment following the first year, and remains relatively small in the the high casualty treatment except for the sixth and seventh years, when the sample size has become relatively small; in a dynamic panel set-up, it is thus likely that we would see a similar decrease in uncertainty over time, offering suggestive evidence that the divergent effects of casualties across the two setups is at least partially due to differences between the panel- and one-shot designs.

\section{DisPoSitionAL AND DEMOGRAPHIC RESULTS}

Thus far, we have seen that participants respond similarly to the costs of backing down across the two experiments, but not the costs of fighting, likely due to the divergent setups of the experiments, rather than characteristics of the samples. What about the dispositional characteristics?

One of the striking results from the lab experiment was that the substantive magnitude of the dispositional variables was larger than those of the situational ones; although it is possible that this imbalance in effect size was due to the lab experiment employing better dispositional measures than situational manipulations, the results nonetheless suggested that strictly situational accounts of resolve that focus solely on the nature of the environment that actors face miss an important part of the story. Given the greater uncertainty present in the survey experiment, we might imagine that the dispositional variables should play an even greater role here (Kertzer and McGraw, 2012).

The first model in Table 4.2 presents the results of the experimental treatments

\footnotetext{
${ }^{12}$ On variance as a measure of uncertainty, see Alvarez and Brehm (1995); Reed (2003); Bas (2012); Mattiacci and Braumoeller (2012).
} 
alongside time preferences, risk aversion, loss aversion, honor culture, and behavioral self-control. The second model adds a series of demographic controls (party identification, gender, race, education, and income). The substantive effects are also presented with 90\% and 95\% confidence intervals in Figure 4.6.

The treatment effects in model (1) are largely similar to those estimated in Table 4.1 - reputation costs are the only significant treatment effect, bolstering participants' resolve by $14.4 \%$, while anticipated costs narrowly escape statistical significance, raising resolve by $9.9 \%$. More interesting, though, are the dispositional effects. A change in time preferences from $\delta=0$ to $\delta=1$ is associated with a $40.9 \%$ increase in resolve; participants scoring as more patient in general thus also display more patience in the intervention scenario. As in the lab experiment, risk aversion has a significant quadratic effect, plotted in Figure $4.7 .^{13}$ In the lab experiment, where participants were moved both by the costs of fighting and by the costs of backing down, we saw that both highly risk averse participants and relatively risk acceptant participants displayed a greater level of resolve. Here, we see a different quadratic relationship, in which highly risk averse participants are more likely to stay in than relatively risk acceptant ones, who are more willing to cut and run. The impact of loss aversion, although narrowly escaping statistical significance until demographic factors are controlled for in model 2, is suggestive: an increase from the minimum to the maximum level of loss aversion is associated with a $24.5 \%$ increase in resolve. Thus, for both risk attitudes in the domain of gains, and risk attitudes in the domain of losses, we find evidence that increased acceptance of risk and loss is associated with decreased resolve. In this sense, we once again find no support for the classic Schelling hypothesis that resolve should linearly increase with risk acceptance, offering further ammunition for the argument that both pressing on in a quagmire and backing down in the face of reputational consequences can be perceived as risky.

The behavioral self-control score also offers some provocative findings: mov-

\footnotetext{
${ }^{13}$ Likelihood ratio tests indicate the quadratic fits better than a linear one $(p<0.02)$, and fails to find evidence that it is outperformed by a nonparametric smoothing spline $(p<1.00)$.
} 
Table 4.2: Dispositional determinants of resolve

\begin{tabular}{|c|c|c|c|c|}
\hline & \multicolumn{2}{|r|}{ (1) } & \multicolumn{2}{|r|}{ (2) } \\
\hline & $\beta$ & Hazard Ratio & $\beta$ & Hazard Ratio \\
\hline \multirow[t]{2}{*}{ Anticipated costs } & -0.104 & $-9.9 \%$ & -0.090 & $-8.6 \%$ \\
\hline & $(0.065)$ & {$[-19.1 \%, 0.34 \%]$} & $(0.066)$ & {$[-18.0 \%, 1.8 \%]$} \\
\hline \multirow[t]{2}{*}{ Reputation costs } & -0.155 & $-14.4 \%$ & -0.155 & $-14.4 \%$ \\
\hline & $(0.064)$ & {$[-23.0 \%,-4.8 \%]$} & $(0.065)$ & {$[-23.0 \%,-4.7 \%]$} \\
\hline \multirow[t]{2}{*}{ Casualties } & -0.083 & $-8.0 \%$ & -0.057 & $-5.5 \%$ \\
\hline & $(0.065)$ & {$[-17.2 \%, 2.3 \%]$} & $(0.065)$ & {$[-15.1 \%, 5.1 \%]$} \\
\hline \multirow[t]{2}{*}{ Order } & -0.050 & $-4.8 \%$ & -0.035 & $-3.4 \%$ \\
\hline & $(0.064)$ & {$[-14.4 \%, 5.8 \%]$} & $(0.065)$ & {$[-13.2 \%, 7.4 \%]$} \\
\hline \multirow[t]{2}{*}{$\delta$} & -0.525 & $-40.86 \%$ & -0.564 & $-43.1 \%$ \\
\hline & $(0.201)$ & {$[-57.5 \%,-17.71 \%]$} & $(0.201)$ & {$[-59.1 \%,-20.8 \%]$} \\
\hline \multirow[t]{2}{*}{ Risk aversion } & -0.474 & * & -0.507 & * \\
\hline & $(0.327)$ & * & $(0.312)$ & * \\
\hline \multirow[t]{2}{*}{ Risk aversion $^{2}$} & 0.549 & * & 0.591 & * \\
\hline & $(0.300)$ & * & $(0.296)$ & * \\
\hline \multirow[t]{2}{*}{ Honor culture } & 0.025 & $2.5 \%$ & -0.010 & $-1.0 \%$ \\
\hline & $(0.173)$ & {$[-22.9 \%, 36.3 \%]$} & $(0.177)$ & {$[-25.9 \%, 32.4 \%]$} \\
\hline \multirow[t]{2}{*}{ Loss aversion (logged) } & -0.011 & $1.07 \%$ & -0.013 & $-1.3 \%$ \\
\hline & $(0.007)$ & {$[-2.2 \%, 0.1 \%]$} & $(0.007)$ & {$[-2.5 \%,-0.2 \%]$} \\
\hline \multirow[t]{2}{*}{ Behavioral self control } & -0.251 & $-22.21 \%$ & -0.227 & $-20.3 \%$ \\
\hline & $(0.147)$ & {$[-39.0 \%,-0.9 \%]$} & $(0.152)$ & {$[38.0 \%, 2.3 \%]$} \\
\hline \multirow{2}{*}{\multicolumn{3}{|c|}{ Party ID }} & -0.559 & $-42.8 \%$ \\
\hline & & & $(0.112)$ & {$[-52.4 \%,-31.3 \%]$} \\
\hline \multirow[t]{2}{*}{ Male } & & & -0.169 & $-15.6 \%$ \\
\hline & & & $(0.066)$ & {$[-24.3 \%,-5.8 \%]$} \\
\hline \multirow[t]{2}{*}{ White } & & & -0.002 & $-0.2 \%$ \\
\hline & & & $(0.081)$ & {$[-12.6 \%, 14.0 \%]$} \\
\hline \multirow[t]{2}{*}{ Education } & & & -0.115 & $-10.9 \%$ \\
\hline & & & $(0.147)$ & {$[30.0 \%, 13.6 \%]$} \\
\hline \multirow[t]{2}{*}{ Income } & & & 0.277 & $31.9 \%$ \\
\hline & & & $(0.159)$ & {$[1.6 \%, 71.2 \%]$} \\
\hline \multirow[t]{2}{*}{ Age } & & & -0.003 & $-0.3 \%$ \\
\hline & & & $(0.002)$ & {$[-0.7 \%, 0.0 \%]$} \\
\hline $\mathrm{N}$ & & 973 & & 973 \\
\hline
\end{tabular}


Figure 4.6: Coefficient plot of situational and dispositional correlates of resolve (1)

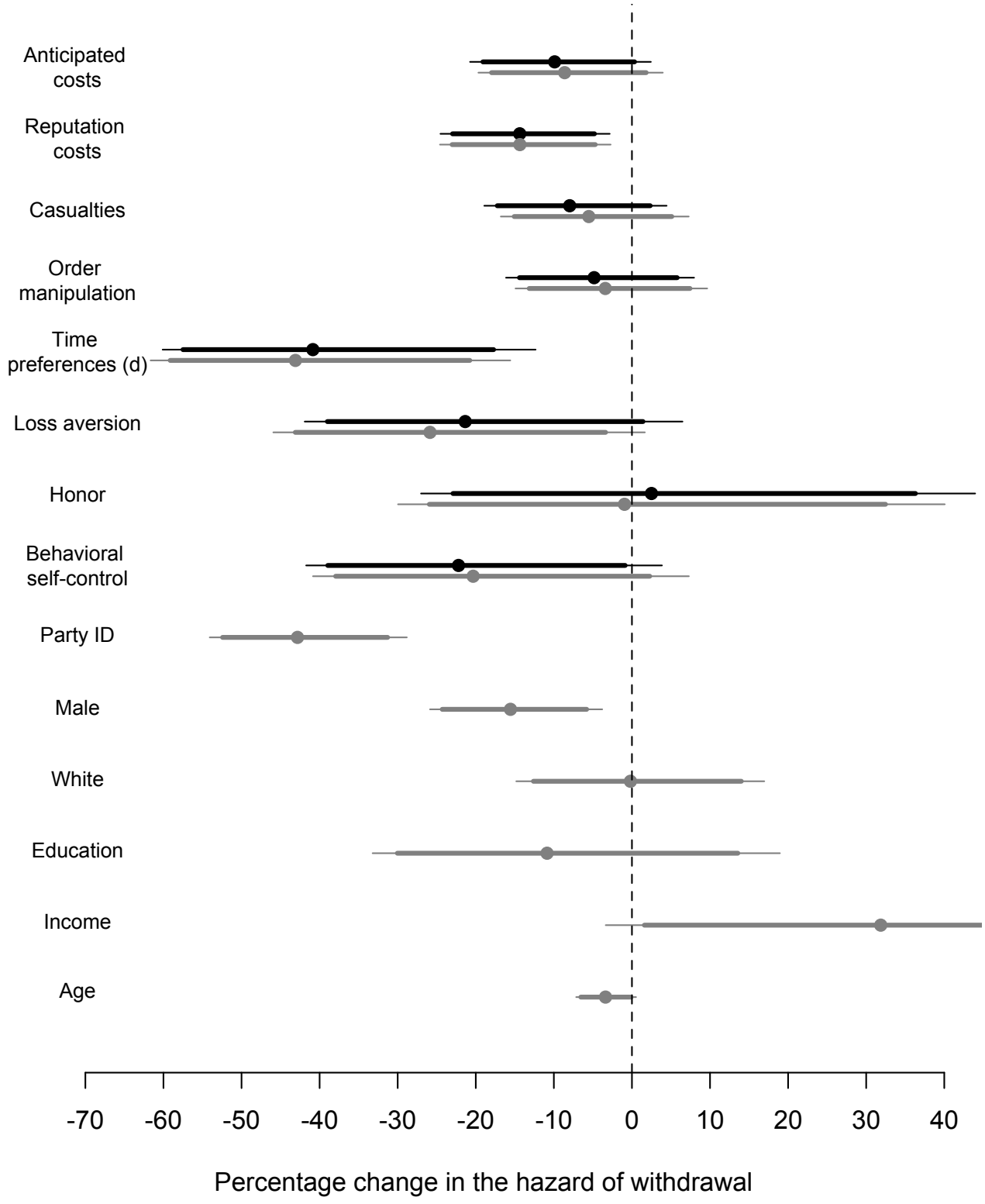

Point estimates of percentage change in the hazard ratio (with $90 \%$ and $95 \%$ confidence intervals) incurred by moving from a 1-unit change in each variable - which corresponds to a change from the minimum to the maximum value, except for $\delta$, for which a one-unit change from 0 to 1 is substantively meaningful, and age, where the hazard ratio depicts a 10 year increase. Negative values indicate a decreased probability of withdrawal, and thus, greater resolve. Darker estimates from model 1 in Table 4.2; lighter estimates from model 2. 
Figure 4.7: Quadratic effect of risk aversion ( $\mathrm{N}=2500$ simulations)

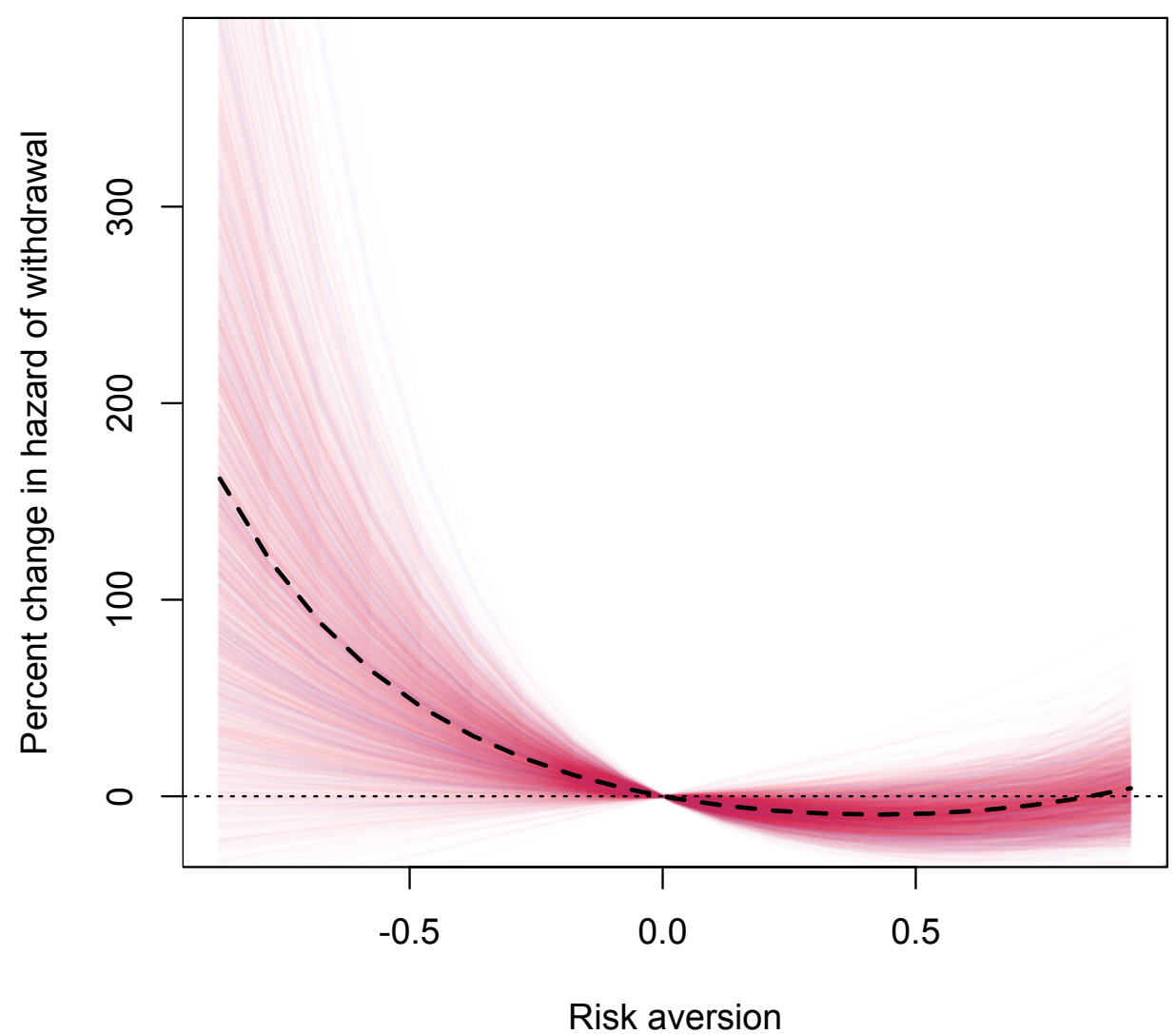

The quadratic effect of risk aversion on participants' resolve, where each red line depicts a draw from a multivariate normal distribution, given $\widehat{\beta}$ and $\widehat{v a r}(x)$ from model 1 of Table 4.2 , such that darker lines indicate a more concentrated probability mass. The dashed black line represents the mean prediction.

ing from the minimum to the maximum score is associated with a $22.2 \%$ increase in participants' resolve in the military intervention scenario: those participants who display self-control in their daily lives as measured by their dietary, exercise and smoking habits also displayed more resolve in the military intervention. Since these habits are also likely to correlate with socio-economic characteristics, it is important to note that the effect of behavioral self-control largely persists even when demographic characteristics like education, income, gender and race are controlled for, thus offering further evidence that personal and political will are related to one 
another.

Just as important as the factors that achieve statistical significance are those that fail to do so. In the lab experiment in Chapter 3, honor culture was initially significantly associated with participants' resolve, but this effect dissipated once gender and party ID were controlled for. With the new measure of honor used in the survey experiment, we find that honor almost uniformly lacks statistical significance, even without demographic controls. ${ }^{14}$ As Figure 4.8 illustrates, honor culture appears to have a conditional relationship with resolve: amongst those participants who were initially opposed to the intervention, honor boosts resolve by $40.5 \%$, while it has no significant relationship in the pro-intervention subsample, but given the fragility of these results, little should be made of it. ${ }^{15}$ Similarly, although the general risk orientation measure is borderline significant as a predictor of resolve in a simple model with just the four treatment variables, it quickly loses significance once other predictors are added, and is omitted from the models presented in Table 4.2.

Finally, the second model in Table 4.2 includes a series of demographic controls, whose inclusion neither substantively changes the treatment effects nor those of the dispositional variables, but which are nonetheless interesting in their own right. Party identification powerfully predicts resolve: strong Republicans are $42.8 \%$ less likely to withdraw at any point in time than strong Democrats, even when controlling for a host of dispositional factors. Men are 15.6\% more resolved than women, and older participants are more resolved than younger ones: a ten year increase in age is associated with a $3.4 \%$ decrease in the probability of withdrawal. Interestingly, income has the only statistically significant positive coefficient: moving from the lowest to the highest income grouping in the sample is associated with a $31.9 \%$ decrease in resolve. This effect is notable both because of its substantively large magnitude, but also because previous research has found that higher income

\footnotetext{
${ }^{14}$ Supplementary analyses indicate that unlike its lab experiment counterpart, the measure of honor employed here is uncorrelated with party identification $(r=-0.01, p<0.80)$ and political ideology $(r=0.00, p<0.99)$, suggesting it is tapping into a somewhat different construct.

${ }^{15}$ The coefficient estimate in the pro-intervention subsample is positive, but not statistically significant $(p<0.22)$.
} 
Figure 4.8: Coefficient plot of situational and dispositional correlates of resolve (2)

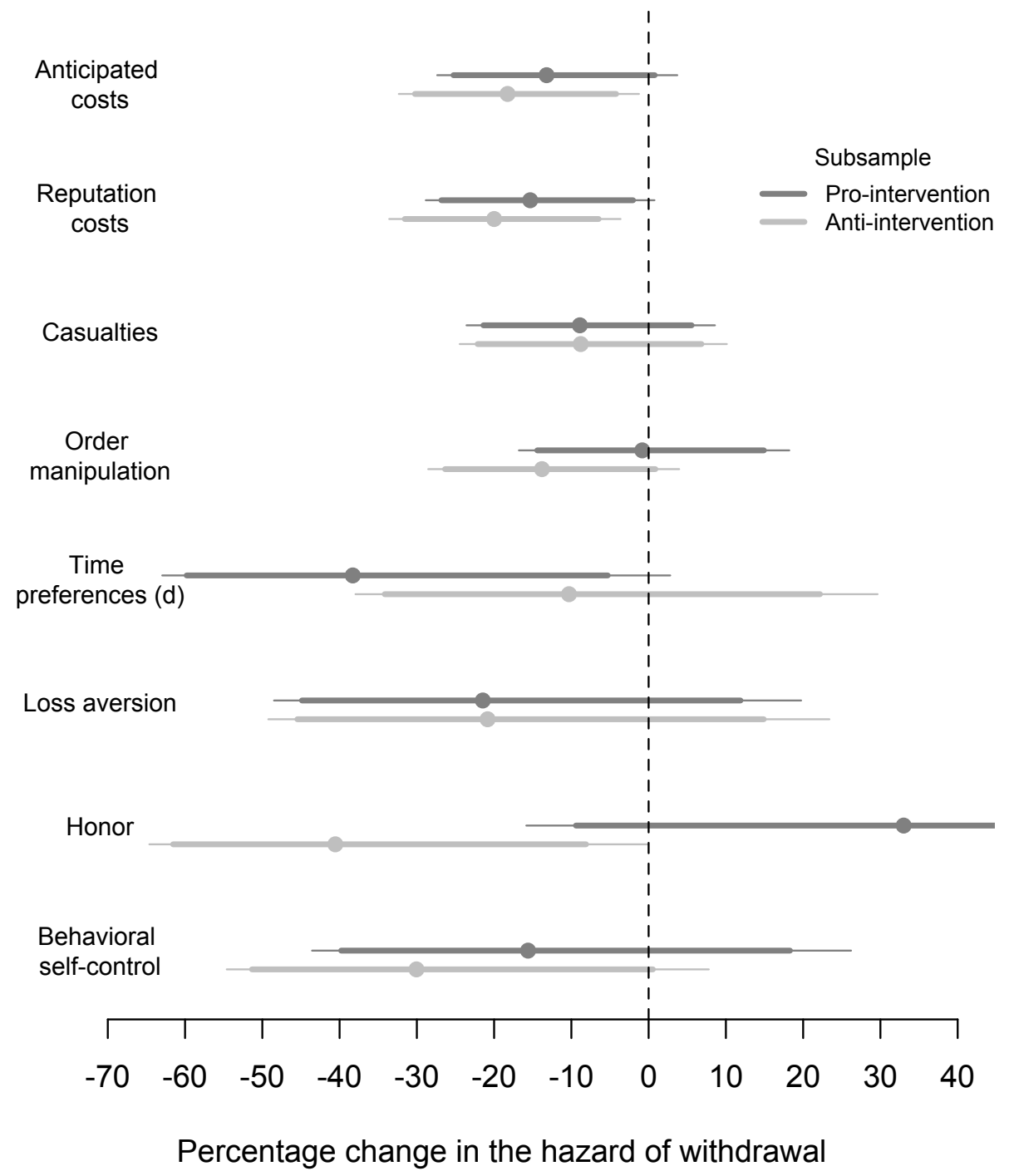

Point estimates of percentage change in the hazard ratio incurred by moving from a 1-unit change in each variable (with $90 \%$ and $95 \%$ confidence intervals) - which corresponds to a change from the minimum to the maximum value, except for $\delta$, for which a one-unit change from 0 to 1 is substantively meaningful. Negative values indicate a decreased probability of withdrawal, and thus, greater resolve. Darker estimates are from a model estimated on those participants who initially supported the intervention; lighter estimates are from a model estimated on those participants who initially opposed the US going in. 
is positively associated with support for more active or extroverted foreign policies (Russett and Nincic, 1976; Wittkopf, 1990, p. 39; Kertzer, 2013), whereas we find the opposite here. ${ }^{16}$ Since the effect's statistical significance weakens when party ID is not also being controlled for, however, we should be wary of drawing too many conclusions from it.

\section{INTERACTIONIST RESULTS}

Finally, we find a number of theoretically meaningful interaction effects, one of which reproduces a pattern present in the lab experiment results, and two of which produce new effects. In the lab experiment, time preferences were found to moderate the human costs of fighting, and risk preferences moderated both the human costs of fighting and the reputational costs of backing down. Given that the casualty treatment lacked significant effects due to the one-shot format of the survey experiment, it should not be surprising that the interaction effects with casualties in the lab experiment do not materialize in the survey experiment. However, we once again find a weakly significant $(p<0.069)$ risk $\mathrm{x}$ reputation cost interaction, this time using the measure of dispositional risk orientation. As the combined coefficient and 95\% confidence bands in Figure 4.9 illustrate, participants who selfdescribed as relatively risk averse were more sensitive to the reputational costs of backing down: it is only for those participants who rate themselves at the midpoint of the risk orientation scale or below that we can be $95 \%$ confident that the reputation cost manipulation boosts resolve, offering further evidence that backing down in the face of reputational consequences can be seen as risky.

Just as in the lab experiment, honor culture fails to moderate the impact of reputation costs; in the survey experiment, however, we see that honor moderates the impact of the anticipated cost treatment $(p<0.009)$. As shown in Figure 4.10,

\footnotetext{
${ }^{16}$ To be sure, an active or extroverted foreign policy is not completely identical to a resolved one, but if we think about resolve in the context of Klingberg's (1952) mood theory, the idea of "losing heart" in a military intervention is conceptually similar to a decline towards foreign policy introversion.
} 
the higher participants scored in honor culture, the more being warned in advance that the expected costs of fighting would be high bolstered resolve; among those participants with honor scores a little under 0.6, we fail to find evidence with 95\% certainty that the anticipated cost treatment bolsters their resolve whatsoever.

Finally, the behavioral self-control factor scores did not significantly moderate any of the treatments in theoretically meaningful ways, ${ }^{17}$ but one of the items used to generate the behavioral self-control score - participants' smoking habits - displays a preliminary, but intriguing interaction effect with the casualty manipulation. As illustrated in Figure 4.11, those participants who reported smoking every day displayed far less resolve in response to the casualty treatment than those participants who display the self-control to either limit their smoking, or avoid smoking altogether. The fact that the interaction is produced with smoking behavior but not the behavioral self-control score means that the findings should be interpreted as tentative, but given the number of demographic characteristics that fail to reveal treatment heterogeneity with the casualty treatment in the supplementary analyses in Appendix $C$, the effect shown here is striking, particularly because it grows rather than shrinks in statistical significance once socioeconomic demographic controls are present.

\subsection{Conclusion}

In this chapter, I sought to take another look at individual-level microfoundations for resolve, presenting the results from an online survey experiment that, like the lab experiment presented in the previous chapter, manipulated situational features of a hypothetical military intervention and measured a series of dispositional characteristics borrowed from social psychology and behavioral economics. The survey experiment borrowed much of its instrumentation from its lab experiment predecessor, but also included a number of modifications, including a slightly differ-

\footnotetext{
${ }^{17}$ A significant interaction effect exists between behavioral self-control and the anticipated cost treatment, but the signs are the opposite of what we would expect them to be.
} 
Table 4.3: Comparison of results across experimental formats

\begin{tabular}{|c|c|c|}
\hline & \multicolumn{2}{|c|}{ Effect on resolve displayed } \\
\hline Variable & Lab experiment & Survey experiment \\
\hline \multicolumn{3}{|l|}{ Situational variables } \\
\hline Anticipated costs & $+18.3 \%$ & $+7.6 \%$ \\
\hline Reputation costs & $+17.5 \%$ & $+12.2 \%$ \\
\hline Casualties & $-31.9 \%$ & $+6.8 \%$ \\
\hline \multicolumn{3}{|l|}{ Dispositional variables } \\
\hline$\delta$ & $+65.8 \%$ & $+40.9 \%$ \\
\hline Risk aversion $(\rho)$ & Quadratic & Quadratic \\
\hline Honor culture & $+67.8 \%$ & $-2.5 \%$ \\
\hline Trait / behavioral self-control & $+81.5 \%$ * & $+22.2 \%$ \\
\hline \multicolumn{3}{|l|}{ Interaction effects } \\
\hline Time preferences $x$ casualties & $\checkmark$ & \\
\hline Risk preferences $x$ casualties & $\checkmark$ & \\
\hline Risk preferences $\mathrm{x}$ reputation costs & $\checkmark$ & $\checkmark$ \\
\hline Honor $x$ anticipated costs & & $\checkmark$ \\
\hline Smoking habits $\mathrm{x}$ casualties & & $\checkmark$ \\
\hline $\begin{array}{l}\text { Effects significant at } \alpha=0.1 \text { bolded } \\
\text { from Table } 3.1 \text {, dispositional effect } \\
\text { in the survey experiment from T } \\
\text { Table } 4.2{ }^{*} \text { Conditiona }\end{array}$ & $\begin{array}{l}\text { Situational effect } \\
\text { from Tables } 3.3- \\
\text { le } 4.1 \text {, and dispc }\end{array}$ & $\begin{array}{l}\text { in the lab experiment } \\
\text { 4. Situational effects } \\
\text { tional effects from }\end{array}$ \\
\hline
\end{tabular}

ent reputation cost treatment, a dispositional risk orientation measure, a different honor orientation measure, a behavioral self-control measure, and a different measure of the dependent variable. In this sense, the survey experiment offers a chance to explore two different types of generalizability: examining whether effects hold up amongst a less homogenous sample, and observing how relationships amongst the variables change when we employ slightly different measures of the key concepts.

Because the two studies differ in multiple ways, a direct pairwise comparison is somewhat complicated, but Table 4.3 highlights the key results. First, the human costs of fighting play a smaller role in the survey experiment than the lab experiment - likely due to the former's one-shot structure, whereas the reputational costs of backing down play a larger role in the survey experiment, stemming in part from the stronger reputation manipulation. These findings make clear that participants 
are swayed by both the costs of fighting and the costs of backing down, although how much participants respond to each is partially a function of how the treatments are designed. In this sense, the findings should make us wary of attempts to draw inferences about the relative importance of different types of costs of war from experimental studies, since so much depends on the dosage of the experimental treatments. That said, given how central casualties are to our theories of public opinion about the use of force (Mueller, 1971; Gartner, 1997), however, the fact that it plays a relatively weak role in the survey experiment - its treatment effect is around half the size of the reputation cost treatment - is notable.

Second, we find more consistently significant results with the dispositional variables. In both the lab and survey experiment, participants with longer shadows of the future displayed more resolve in the intervention scenario; similarly, we find across both experiments that risk has a quadratic relationship with resolve, although the shapes of the curves vary across the two experiments. In the lab experiment, where participants seem concerned both about the costs of fighting and (to a lesser extent) about the costs of backing down, we see that both relatively risk acceptant and highly risk averse participants are more likely to display resolve; in the survey experiment, on the other hand, where participants seem to be concerned mostly about the reputational costs of backing down, we see that highly risk acceptant participants are far less resolved than highly risk averse ones. In modeling both risk aversion and loss aversion, the survey experiment also shows us that risk attitudes predict resolve in both the domain of gains and the domain of losses. Honor's effects are weaker in the survey experiment than the lab experiment, and self-control plays a large role in both, albeit a slightly different one. In the lab experiment, where perseverance is used as a measure of trait self-control, self-control has a significant conditional effect upon resolve, in that among those participants who initially approved of the intervention, perseverance strongly predicts how much resolve participants displayed. In the survey experiment, where self-control was measured through behavioral report items, we see that self-control 
has a significant unconditional effect on resolve.

Third, only one of the interaction effects from the lab experiment reproduces in the survey experiment - risk $\times$ reputation cost - and even then, it is worth noting that the interaction in the survey experiment holds with the dispositional measure of risk attitudes rather than the lottery measure used in the lab. In this sense, the survey experiment offers further evidence of dispositional characteristics moderating the impact of situational features, and finds evidence for some theoretically meaningful interaction effects not detected in the lab, but also shows the extent to which our findings are often shaped by the instrumentation we employ.

More broadly, these experiments suggest that we should think of resolve in military interventions as a function of both stakes and traits - both situational variables, and dispositional ones. ${ }^{18}$ The advantage of the experimental methods employed in these two chapters is that they allow us to cleanly manipulate situational features of military interventions and directly measure dispositional characteristics of participants, while letting us study resolve as a dependent variable by observing how changes in these situational and dispositional variables affects how much resolve participants display. If we were solely interested in explaining resolve in the context of public opinion about military interventions, we might end our investigation here, having shown that political will is more than just a figure of speech, and that the same factors that behavioral economists and social psychologists use to predict willpower in our daily lives also moderate the impact of the cost of war, and explain variation in the public's resolve in military interventions.

However, the experimental results presented over these past two chapters also present us with another possibility. As I argued in Chapter 1, one of the chief reasons why political scientists have had such a hard time studying resolve is because we have often been forced to tautologically infer resolve via the effects we are trying to use it to explain - the argument, for example, that the US lost in Vietnam because Washington was less resolved than Hanoi, so to win in war, actors need to

\footnotetext{
${ }^{18} \mathrm{AIC}$ scores confirm that despite the reduction in parsimony, the dispositional and demographic models presented in Table 4.2 are superior to the purely situational model from Table 4.1.
} 
be more resolved. The reason why both pundits and scholars have been so quick to reach for this tautology is because resolve in the real world is not directly observable, so we have been forced to grapple with resolve's effects by inferring resolve based on what we can observe. Thus, the next chapter alters the focus of our analyses. Having used the controlled experimental environment to study resolve's causes, we then build on these individual-level microfoundations to study resolve's consequences. Doing so not only allows us to move beyond the experiment's focus on resolve in the context of public opinion to look at resolve with respect to elite decision-makers, but also allows us to test whether resolve has the effects on victory we often claim it does - using measures of resolve derived independently of the outcomes we are using it to explain. 
Figure 4.9: Risk orientation $\mathrm{x}$ reputation costs combined coefficient plot

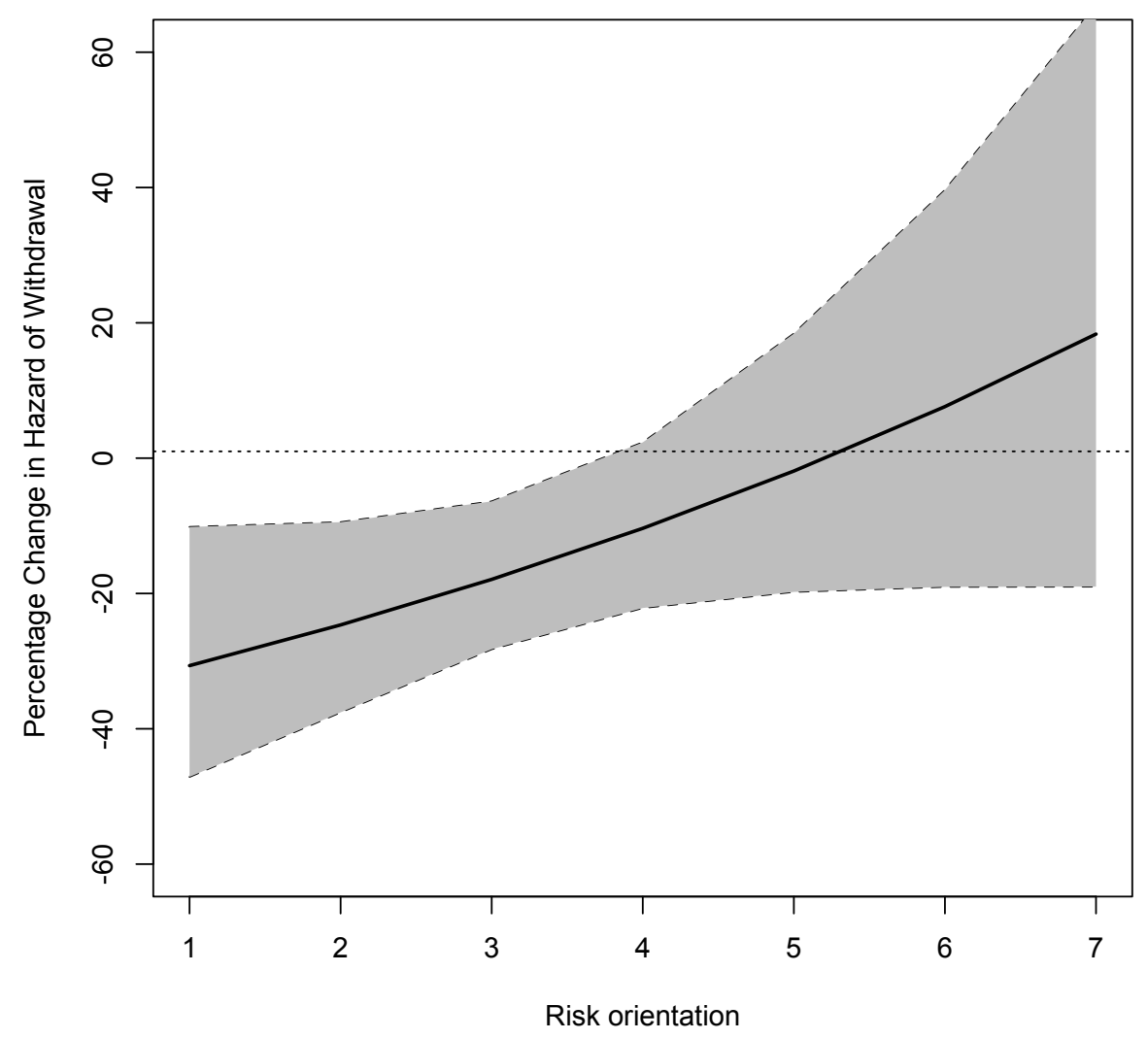

Risk orientation moderates the impact of reputation costs: participants who self-described as relatively risk averse were more sensitive to the reputational costs of backing down, similar to the risk aversion $\mathrm{x}$ reputation interaction effect detected in the lab experiment. The dark line indicates the point estimate of the combined coefficient for the conditional effect, while the shaded grey depicts $95 \%$ confidence bands calculated using draws from a multivariate normal. 
Figure 4.10: Honor culture $\mathrm{x}$ anticipated costs combined coefficient plot

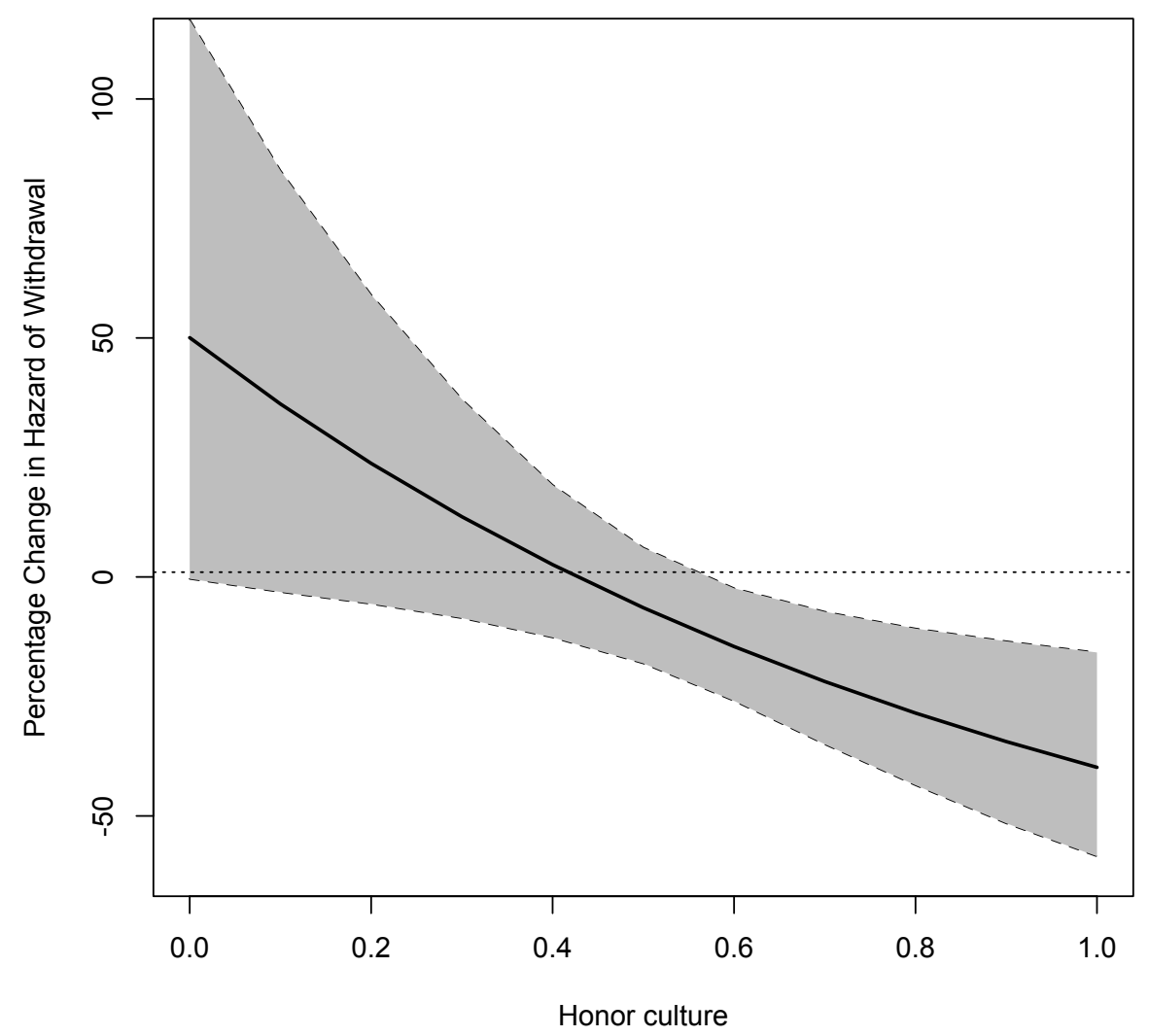

Honor culture moderates the impact of the anticipated costs of fighting: participants high in honor culture are more sensitive to the expected costs of fighting. The dark line indicates the point estimate of the combined coefficient for the conditional effect, while the shaded grey depicts 95\% confidence bands calculated using draws from a multivariate normal. 
Figure 4.11: Smoking behavior $\mathrm{x}$ casualties combined coefficient plot

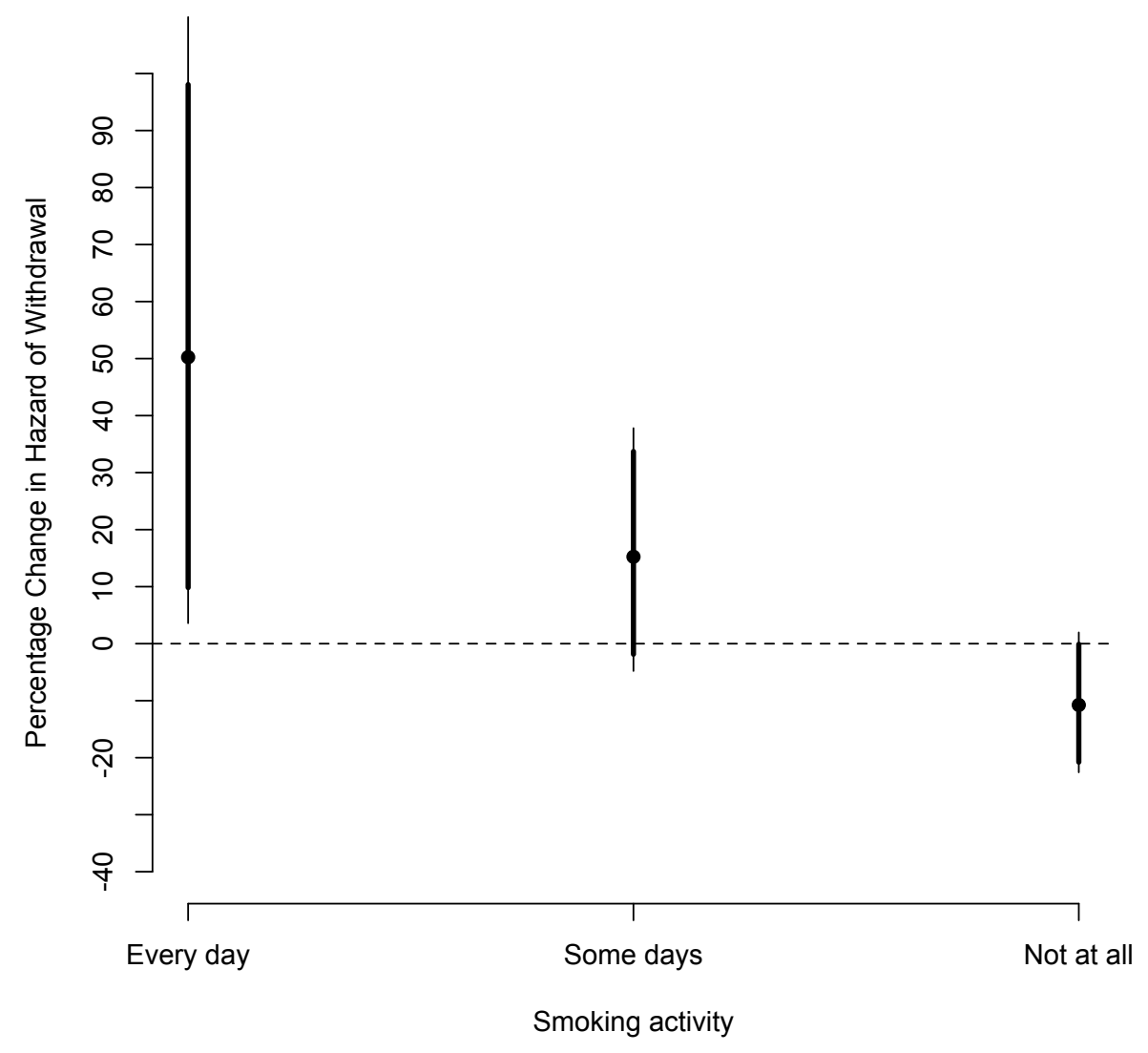

Smoking behavior moderates the impact of casualties: although the behavioral self-control score did not significantly moderate the impact of the casualty treatment, one of its component elements, smoking behavior, did. Participants who smoke every day were far more sensitive to the casualty manipulation than those who smoked some days, or not at all. Effects depicted with $90 \%$ and $95 \%$ confidence intervals calculated using draws from a multivariate normal. 


\section{Chapter 5: Resolve in Great Power Military Interventions, 1946-2003}

The previous two chapters have employed lab and survey experiments to study resolve as a dependent variable: by manipulating situational features of military interventions and measuring dispositional characteristics of participants in a controlled experimental setting, we are able to make fairly fine-grained distinctions about the associations between dispositional traits and resolve, and draw causal inferences about the relationship between different types of costs of war and resolve. However, these experiments do not serve as the endpoint of the analyses. In this chapter, I treat the experimental results as theoretical microfoundations for a Boolean statistical model of great power military interventions in the post-WWII era. Having already established evidence for situational and dispositional determinants of resolve with experimental data at the individual-level, I operationalize these same variables with observational data at the country- and leader-level to construct composite situational and dispositional measures of the resolve of the great powers in military interventions from 1946-2003. The results of my analyses suggest that the situational and dispositional determinants of resolve also positively predict the probability of victory, but at different levels of analysis: conflict outcomes appear to be a function of situational determinants of resolve at the country-level, but dispositional determinants of resolve at the leader-level. In this sense, the large-N analyses both support and enrich the results from the preceding two chapters.

Since the type of analysis I perform here is somewhat unusual, I begin with a discussion of the importance of microfoundations in IR theory, before suggesting that in treating resolve as the function of two latent variables, Boolean statistics 
offers a solution for a problem that has bedeviled security international security scholars for some time: how to test the effects of resolve on conflict outcomes without tautologically inferring resolve from the outcomes it is being used to explain. I then discuss the construction of the dataset and present results from two types of Boolean analyses: a series of conjunctural models in resolve is situational and dispositional, and a substitutable model in which resolve is situational or dispositional. I then show that these Boolean models display far higher predictive power than a traditional additive model, and conclude by discussing some of the implications of the findings.

\subsection{Microfoundations and latent variables}

Especially once we move away from grand theories (e.g. Wallerstein 1974) towards the "middle-range theories" (Merton, 1968) that dominate the study of international security, a significant proportion of our accounts of international politics rely on microfoundations - individual-level "cogs and wheels" posited to act as mechanisms linking cause and effect (Elster, 1989, 3). ${ }^{1}$ There has been a considerable push for microfoundations in the social sciences in the past several decades, especially by "strong" methodological individualists, for whom theories that fail to specify individual-level mechanisms are "lazy and frictionless" (Elster, 1982, 453). ${ }^{2}$ Although macro-theorists warn that the search for microfoundations can sometimes

\footnotetext{
${ }^{1}$ The term "microfoundation" tends to be used in a wide variety of ways. Achen $(2002,437)$, for example, uses microfoundations to refer to "a formal model of the behavior of the political actors under study" to guide our use of particular statistical estimators, a definition that need not imply that the mechanism connecting the explanans and explanandum exists at a particular level of analysis. Instead, I use microfoundations here to refer to an analytic strategy where one explains outcomes at the aggregate level via dynamics at the individual level, consistent with how it is used in economics ("to refer to schemes which can be broadly regarded as attempting to reduce macroeconomics to microeconomics" (Nelson, 1984, 575)) and sociology, which justifies the search for microfoundations by suggesting that "social phenomena... are in principle explicable in ways that only involve individuals - their properties, their goals, their beliefs, and their actions." (Elster, 1985, 5). Since the key logic behind the search for microfoundations is to identify the micro-level processes that explain how the phenomena of interest occur, microfoundations are often associated with causal mechanisms, but as Gerring (2007) points out, not all causal mechanisms implicate microfoundations.

${ }^{2}$ On methodological individualism more generally, see Udehn (2001).
} 
be problematic - some causal mechanisms exist only at the macro-level (e.g. supply and demand in economics, natural selection in evolutionary biology), while multiple realizability means that many macro-level outcomes cannot simply be reduced to micro-level causes (Schelling 1978, 13, Wendt 1999, 150-157) — it is worth noting that even structural and institutionalist theories frequently rely on microlevel assumptions. ${ }^{3}$ Rational choice theory, for example, is often used to showcase the power of institutions (e.g. Shepsle and Weingast 1981), but also relies upon assumptions about the content and shape of actors' utility functions (Simon, 1985). Realist theories of international politics - especially in Waltz's (1979) formulation — are often viewed as structural theories par excellence (Wendt, 1987), but also rely on particular sets of assumptions about the behavioral consequences of uncertainty and fear, for example (Kertzer and McGraw, 2012). In this respect, one need not be a strong methodological individualist to argue that microfoundations matter (Udehn 2001, 336, Hedström and Ylikoski 2010, 59-60): complexity theory, for example, is built upon the premise that one cannot explain systems solely by reference to the behavior of each of its components (Miller and Page, 2007, 3) - and thus, the rejection of methodological individualism - yet complexity scholars in IR frequently use agent-based modeling to show how the emergent properties of systems depend on the parameters of agents' preferences and behaviors (Axelrod, 1997; Cederman, 1997).

A major challenge with the current state of the study of international security, then, is not that our theories lack microfoundations, but rather, that i) we often fail to specify what these microfoundations are - democratic peace theory, for example, is "an empirical regularity in search of a theory" (Hopf, 1998, 191), and ii) rarely test them. Pape's (1996) pioneering study of the effects of coercive bombing on military outcomes, for example, relies on a particular set of micro-level assumptions about how individual civilians respond to coercion, but whose validity is seldom

\footnotetext{
${ }^{3}$ For applications of multiple realizability to IR, see Most and Starr 1984; Thompson 2003. The study of power laws and scale invariance (Richardson, 1948; Cederman, 2003; Clauset, Young, and Gleditsch, 2007) offers another good example of multiple realizability, and theories constructed with macro- rather than microfoundations.
} 
tested (Condra and Shapiro, 2012; Lyall, Blair, and Imai, Forthcoming); the same could be said of audience cost theory and its vision of a monolithic public responding in unison like the chorus in a Greek tragedy (Trager and Vavreck, 2011).

An equivalent problem exists with the study of resolve. As I suggested in Chapter 1 , although resolve is one of our central explanatory variables in the study of international security, we have very little sense of where resolve itself actually comes from: we lack microfoundations. This absence of microfoundations is problematic both because of theoretical underspecification, and empirical misspecification: we not only use the concept inconsistently, but we also have difficulty measuring it with observational data, such that we either tend to relegate our favorite independent variable to the error term, or infer it tautologically from the outcomes we are using it to explain, thereby turning it into a catch-all residual category used ex post to explain otherwise unexplainable conflict outcomes.

The chief difficulty political scientists have faced in studying resolve with observational data is that resolve in the real world is not directly observable: it is a latent variable (Bollen, 1989; Wansbeek and Meijer, 2000). I am not the first person to suggest that resolve is akin to a latent variable; ${ }^{4}$ if resolve was directly observable, actors would have no need for going through the trouble of signaling their resolve, and there would be no incentives for actors to misrepresent their resolve in the first place (Fearon, 1995). In this sense, it is precisely because resolve cannot be directly observed that it plays such an important role in rationalist theories of war. Yet the very unobservable quality that gives resolve such explanatory power in our theories also renders us powerless when we try to test them empirically. Although political scientists regularly study latent variables - personality orientations (Mondak, 2010) regime type (Treier and Jackman, 2008), ideal point models

\footnotetext{
${ }^{4}$ For related arguments, see Clark and Regan (2003), who use a split population model to study opportunity (although not willingness) as an unobserved latent process (see also Xiang (2010)), and Renshon and Spirling (Forthcoming), who model "effectiveness" as a latent variable using BradleyTerry models. Each of these works show that the study of unobservable factors poses important implications for how we test our theories, although both opportunity and effectiveness are closer to capabilities than resolve, per se. For a rather different take on latents in international security, see Rummel (1975a, chap. 9-10).
} 
of state preferences (Voeten, 2000), and so on, the idea being that when a construct is not directly observable, we can model it based on its observable implications resolve is different in that establishing its implications is our goal in the first place. When it comes to regime type, we cannot directly measure how democratic a state is, so we instead measure how free and fair its elections are and treat that as an indirect measure of democracy, since the competitiveness of the executive recruitment process is logically constitutive of what democracy means. The relationship between resolve and military victory, on the other hand, is precisely the empirical question we are trying to prove (Rosen, 1972; Maoz, 1983).

Figure 5.1: Decision tree for studying resolve with observational data

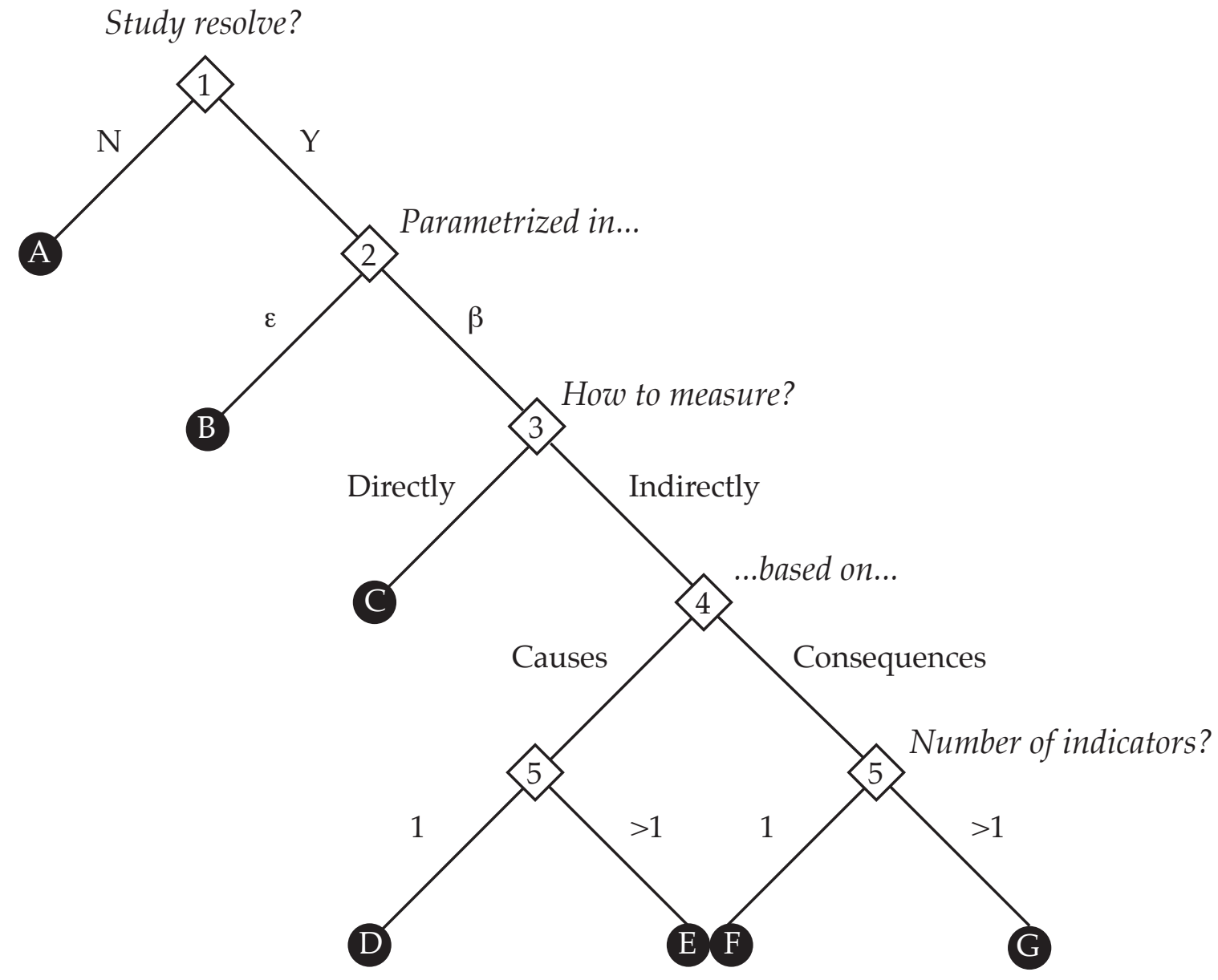

This challenge means that there are at least seven different empirical strategies 
one can adopt facing the prospect of studying resolve with observational data, illustrated by the decision tree in Figure 5.1. First, one can simply decide to avoid studying resolve altogether (option A in Figure 5.1). Merom (2003) takes this route, eschewing "motivational theories" of asymmetric conflict outcomes for being too tautological. Rummel (1975b, 275-276) similarly notes that for many scholars “will is a metaphysical concept, not quite respectable for 'scientific' interest." If one decides to study resolve despite these challenges, the question becomes how to parameterize it. Noting that resolve is "notoriously difficult to measure," Sartori (2005, 91), like Reed (2000), pursues option B, treating resolve as part of the correlated error terms in a unified model of conflict onset and escalation. Although a clever way to handle selection effects in a theoretically-informed manner, relegating resolve to the error term is less helpful in testing its effects, per se - unless we are comfortable assuming that the correlation in the disturbances is due specifically to resolve rather than other omitted variables, hence why Reed $(2000,90)$ is careful to note that resolve is one of a number of potential culprits.

Thus, rather than treating resolve as the error term, a number of works in IR choose to study resolve as a variable in its own right. The subsequent question becomes whether to measure resolve directly (option C) or indirectly (options D$\mathrm{G})$. If resolve is indeed a latent variable, however, then it cannot directly be observed, precluding the possibility of option C. Thus, most of the existing empirical studies of war adopt option F, inferring resolve based on one of its posited consequences: Rosen (1972) and Mueller (1980) infer resolve based on the number of casualties each side is willing to suffer, Maoz (1983) proxies resolve by the highest level of severity of the dispute, Langlois and Langlois (2009) infer resolve based on the percentage of the country's population in the military, Cline (1975) measures resolve via military expenditures, while Sullivan (2007) treats the magnitude of ground troop deployments as an indicator of “commitment." Lewin's (2012) study of resilience in war is illustrative in this regard: his six cases of a lack of "national resilience" are military defeats, while his three cases of national resilience are mil- 
itary victories: resillience might indeed lead to victory, but we would not be able to tell.

Option F faces three potential concerns. First, as argued above, some of these measures of resolve veer dangerously close to tautology and assume axiomatically what should be proven empirically (is it the case that more resolved states suffer more casualties?). Second, as Gelpi and Griesdorf $(2001,638)$ note, there is an important distinction "between demonstrations of resolve and the underlying preferences that may give rise to such demonstrations": escalatory policies may indeed signal resolve, but the resolve these demonstrations are intended to signal may not be sincere - hence why states with low levels of resolve can often prevail in crisis bargaining through successful bluffing (Powell, 1987). If we want to study the effects of resolve - rather than the effects of signals of resolve - we need to find an empirical strategy that avoids conflating the two. Third, if resolve is indeed a latent variable - the motivation for measuring it indirectly in the first place - a single indicator will do a poor job of characterizing the underlying construct.

\subsection{Methods}

Thus, I employ option E: rather than inferring resolve based on its consequences, I study it based on its causes, using the results from the lab and survey experiments as individual-level microfoundations for resolve, which I then gather measures of at the country- and leader-level with observational data. The advantage of this approach is threefold: by focusing on resolve's causes rather than consequences we steer clear of tautology; by turning to microfoundations we offer a more satisfying theoretical explanation, and by employing more than just a single indicator, we can be more confident that we are capturing resolve. ${ }^{5}$ As in the experiment, I focus on resolve in the domain of military interventions, this time focusing on factors shaping the great powers' resolve in their military interventions in the postwar

\footnotetext{
${ }^{5}$ I avoid using the language of "reliability" here for reasons that will be made clear in the discussion of formative indicator models below.
} 
era. I focus on resolve in this realm for both theoretical and substantive reasons. First, it mirrors the micro-level analyses, which also focus on military interventions; whereas the experiments use hypothetical interventions to study the determinants of resolve at the individual level, the large- $\mathrm{N}$ analyses employ real interventions and look across multiple levels of analysis. Second, if resolve is defined as akin to persistence - a steadfastness of purpose that maintains a policy over time despite contrary inclinations or temptations to back down - international interventions are a perfect illustration of resolve at work, as leaders and their publics frequently intervene only to seek an exit option as the pressure mounts (Sullivan, 2008; Polsky, 2010; Koch and Sullivan, 2010).

The theory I am interested in testing is a interactionist one: that resolve is a function of both situational stakes, and dispositional traits. Accordingly, for most of the analyses that follow, I employ a conjunctural Boolean logit model (Braumoeller, 2003), in which $\lambda$ refers to the inverse logit function $\frac{1}{1+e^{-x}}$, the situational determinants of resolve are grouped into one "path" (represented by the vector $B_{S}$ ) and the dispositional determinants of resolve into another (depicted by $B_{D}$ ), with $P_{i, t}$ as the probability that the great power achieves its objectives (and thus, declares victory) at time $t$.

$$
\operatorname{Pr}\left(y_{i, t}=1 \mid \alpha, \mathrm{B}, x_{i, t}\right)=\lambda\left(\alpha_{1}+B_{S} x_{i, t}\right) * \lambda\left(\alpha_{2}+B_{D} x_{i, t}\right)
$$

In this setup, we can think of each vector as a set of situational causes $\left(B_{S}\right)$, and dispositional causes $\left(B_{D}\right)$ of resolve. Thus, resolve in this formulation is represented by two sets of fitted values: a situational resolve score $\left(\alpha_{1}+B_{S} x_{i, t}\right)$, and a dispositional resolve score $\left(\alpha_{2}+B_{D} x_{i, t}\right)$. We can then model conflict outcomes as the product of these two sets of logit-transformed fitted values. ${ }^{6}$ If the situational and dispositional factors that predict resolve in the experimental analyses

${ }^{6}$ The advantage of the Boolean approach is that it allows us to tractably employ an empirical model that corresponds to our interactive theory. As Braumoeller and Carson (2011) show, although it is possible to represent the Boolean logit functional form as a single logit with multiplicative interaction terms, doing so would require a sufficient number of interaction terms so as to render the model extremely difficult to interpret. 
also predict the probability of victory in great power military interventions, we can feel confident that resolve actually predicts conflict outcomes, using measures of resolve derived independently of the outcomes we are using them to explain.

Before discussing the data, I should note four methodological considerations. First, although my empirical strategy is a "two-stage" strategy in the sense that I first employ experiments and then build on these insights with observational data, it is not a formal two-stage model in the tradition of a Heckman-type model where estimates from one stage are directly plugged into a second (e.g. Flores and Nooruddin 2009). ${ }^{7}$ Thus, I do not weight the impact of different sources of resolve based on the experimental results, for example. I avoid this strategy because, as I noted in Chapter 4, a simple comparison between the lab and survey experiment results shows that operationalizing treatments differently produces effects of different magnitude: treatments with stronger "dosages" produce stronger effects. Although the experimental results show that the dispositional variables explained more of the variance in resolve than the situational ones, this could simply be a function of better dispositional measures and weaker treatments. I therefore bypass the question of weighting altogether.

Second, although we often think of resolve in a dyadic fashion such that we can speak of the "balance of resolve" (Maoz, 1983; Powell, 1987), in the analyses that follow I focus solely on the resolve of the intervening power, rather than also looking at the resolve of the target state, or how the resolve of the latter affects the resolve of the former. This is due not only to data limitations, but also for statistical reasons:

\footnotetext{
${ }^{7}$ This distinction also highlights the difference between my strategy and an Instrumental Variable (IV) approach. The purpose of an IV setup is typically to find an instrument $(Z)$ that predicts treatment $T$, but is unrelated to unobserved factors that affect dependent variable $Y$. In our context, resolve is treatment $T$, the attainment of military objectives is dependent variable $Y$, and the dispositional and situational causes of resolve are instrument $Z$. The Boolean strategy I employ here differs from an IV approach in two ways: first, as noted above, I do not employ a two-stage model like two-stage least squares (2SLS) to calculate my estimates. Second, as I note later in the chapter, it is implausible to assert that the situational and dispositional predictors of resolve are uncorrelated with unobserved factors that also affect the probability of victory. The obvious downside to this approach is that it prevents the estimates from being causally identified, but since it is unclear what a plausible instrument for resolve would be in the first place, an IV approach is inapplicable. See Sovey and Green (2011).
} 
given that our composite measures of resolve are (a) likely to have a high degree of measurement error, and (b) driven by the interactions between multiple actor characteristics and the situations actors are facing, conceptualizing resolve in a dyadic fashion risks overprocessing the data until it resembles an Andouille sausage. In Chapter 6, I suggest a number of ways one might examine resolve dyadically with observational data in future research.

Third, although it is plausible that resolve is likely to affect not just the outcome of an intervention, but also whether a party gets involved in the first place, I am not employing a selection model. Not only do statistical solutions for selection bias tend to be quite brittle and plausible instruments hard to come by, but I already have experimental analyses explicitly designed to model this selection process, which finds that not controlling for selection bias actually produces a more conservative test, biasing coefficient estimates downwards, since variables that increase the probability of intervention appear to have a weaker relationship with resolve once the intervention takes place. Thus, if I still find factors like reputation costs have a significant impact, it will increase my confidence in their importance.

Finally, in a more traditional latent variable model of resolve (e.g. option $\mathrm{G}$ in Figure 5.1), we would want to test whether our multiple indicators of resolve are intercorrelated to ensure the reliability of our composite measure, or employ factor analysis to see whether our indicators load onto a single factor. Although this rule holds in classical test theory (in which indicators $x_{i \ldots j}$ are understood to be effects of the latent variable $\xi$ ), the theoretical relationship being tested by option $E$ is akin to what the measurement literature calls a formative indicator model (Edwards and Bagozzi, 2000; Diamantopoulos and Winklhofer, 2001), in that our indicators of resolve are not its effects, but its causes. ${ }^{8}$ Whereas we expect the consequences of a phenomenon to be correlated with one another (e.g. if stable borders affect both

\footnotetext{
${ }^{8}$ For this reason, political scientists who estimate traditional ("effect indicator") structural equation models or factor analyses in situations where our measures are causes rather than consequences of our unobserved constructs are technically estimating the wrong model. The analyses that follow do not employ a structural equation modeling setup, and thus do not formally employ formative indicator models, but because of the considerations discussed below, the analogy holds.
} 
the likelihood of conflict and the likelihood of democracy, democracy and the probability of conflict should be intercorrelated (Gibler, 2007)), this is not necessarily the case with the causes of a phenomenon: for example, although security scholars believe that both the offense-defense balance (Hopf, 1991a) and democratization (Mansfield and Snyder, 1995) cause war, democratization and the offense-defense balance need not be correlated with one another. Thus, the use of factor analysis or reliability analyses would be inappropriate in this context.

\subsection{Data and variables}

The backbone of the analyses that follow is Sullivan and Koch's (2009) Military Interventions by Powerful States (MIPS) dataset, which includes all of the military interventions conducted from 1946-2003 by the five permanent members of the UN Security Council (the United States, the United Kingdom, France, Russia, and China), 126 interventions in all. Military interventions are defined broadly to refer to the use of armed force (involving an official deployment of at least 500 military personnel) in pursuit of political objectives against a foreign adversary (whether a state or non-state actor). Thus, the interventions under investigation here range in severity, from simple displays of force - the US sending troops to the Dominican Republic to stabilize the country following the assassination of Rafael Trujillo in 1961 - to full-on ground combat, such as the Soviet invasion of Afghanistan in 1979. Because a number of our variables of interest are timevarying, I reshape the data such that the unit of analysis is the conflict-month; the data contain 3515 conflict-months in all. The dependent variable is whether the great power has achieved victory at time $t$, based on the ATTAIN variable from the MIPS data, which measures whether the mission's objective was achieved and maintained for at least 30 days. Because of the conflict-month unit of analysis, the variable is recoded such that monthly observations for ongoing interventions are coded 0 , as are the final observations for interventions terminated short of victory. 


\section{Situational VARiables}

\section{The costs of fighting}

Although the lab and survey experiments use casualties as the chief measure of the costs of fighting, two considerations caution against their use here. First are data availability concerns: accurate monthly casualty estimates are easily accessible for some interventions, but not for others. Second, casualties are the ex post costs of fighting; although total casualty figures suffered over the course of the intervention are available, treating the number of casualties sustained at the end of the conflict at time $t+1$ as a predictor of the intervening power's behavior at time $t$ suffers from obvious endogeneity concerns. Instead, I measure the costs of fighting by looking at the level of force employed by the intervening power using Sullivan and Koch's (2009) force type variable from the MIPS data. An ordinal variable, it ranges from a simple display of force to actual ground combat, rescaled from 0-1, like all of the variables discussed below, to facilitate model convergence. In this sense, force type is similar to the anticipated cost manipulation from the lab and survey experiments, which told participants the intervention would require either "minimal" or "substantial use of force." The one respect in which it differs is that since force type here is coded to reflect the amount of force used at the height of the conflict, it is not an entirely ex ante measure, but is nonetheless a better indicator than measures like casualties or the highest number of intervening troops, since intervening powers that have pledged troop deployments are more likely to know in advance whether an intervention will consist of a limited use of force or not, rather than accurately anticipate the precise number of casualties. ${ }^{9}$

\footnotetext{
${ }^{9}$ There is of course a strategic element here, in that larger sized deployments, although more expensive in financial terms, could be deployed because of higher sensitivity to the human costs of war, out of the assumption that employing overwhelming force reduces the likelihood of casualties. On the tradeoff between human and financial costs of war, see Gartzke (2001); Caverley (2009/10).
} 


\section{The costs of backing down}

In the previous chapters, I argued that just as pressing on in a conflict is costly, so too is backing down, and we can think of three different types of costs that actors pay when they back down: the stakes of the conflict (the intrinsic interests for which we were fighting in the first place), reputation costs (the extrinsic interests), and domestic audience costs, which we can think of as a kind of reputational cost paid by leaders to their domestic constituencies. For reasons of tractability, the experiments manipulated reputation costs but kept the stakes fixed, but the use of observational data allows us to examine the impact of multiple types of costs of backing down, at both the country-level and leader-level.

The first measure of the cost of backing down is the distance between the intervening power and the target of the intervention; this serves as a useful measure of intrinsic interests since, as Braumoeller and Carson $(2011,297)$ note, "proximity magnifies the stakes involved and distance attenuates them." To calculate distances, I employ Weidmann, Kuse, and Gleditsch's (2010) cshapes package in R, which uses GIS data to calculate the minimum distance between the intervening state and target at the time of the intervention, thereby accounting for changes in countries' borders over time.

Second, I examine whether the intervention is targeted against a current or former colony of the intervening power. The Soviet Union had more of its reputation at stake in its interventions in Poland in 1956 than it did in its intervention in the Sudanese Civil War in 1971; interventions taking place in current or former colonies will be more likely to implicate reputational costs than those occuring fur-

ther afield, and speak to higher intrinsic interests at stake as well. Here I recode the COLONY variable from the MIPS data, collapsing current and former colonies into one category to produce a dichotomous variable indicating the presence or absence of colonial ties.

Third, the costs of backing down are likely to be higher if there are major economic interests at stake: if the great power is intervening to prop up a regime in 
a friendly trading partner, it is likely to pay heavy consequences if the friendly regime falls. As a measure of economic interests, I calculate trade flows using version 3.0 of the Correlates of War Project's Trade Data (Barbieri, Keshk, and Pollins, 2009). Since I have no theoretical expectations as to whether imports are more important than exports, or vice versa, rather than calculate net trade flows, I add imports and exports together to measure the total trade flow between the intervening state and and location of the intervention. I calculate dyadic trade flows with respect to the location of the intervention rather than the target of the intervention, since the relevant trade flows in the Gulf War, for example, are between the United States and Kuwait, rather than the United States and Iraq. To minimize missing data, I employ either the "nearest neighbor" method if the non-missing observation is within a year of the missing one, or impute a linear trend if no immediate neighbors are found.

Fourth, the costs of backing down in interventions carried out in allied states are likely to be higher: not only are the reputational costs of backing down greater, but so too are the inherent stakes of the intervention. I employ the Alliance Treaty Obligations and Provisions (ATOP) data (Leeds et al., 2002), coding whether an alliance exists between the great power and the location where the intervention is taking place at the time of the intervention - since, to continue the Gulf War example from before, I am more interested in whether the US has an alliance with Kuwait than with Iraq.

Finally, whereas the above measures of the cost of backing down focus on the stakes and reputational costs inherent in the intervention at the country-level, we can also think about stakes and reputation costs existing for the leaders themselves. I follow Croco (2011) in modeling audience costs as a function of leader culpability: leaders are more likely to pay audience costs if they back down in an intervention that they themselves (or a fellow member of their political party) were responsible for initiating, whereas leaders who inherit an intervention from a political opponent are often praised rather than punished when they terminate it, because they 
are seen as extricating a country from a quagmire rather than having gotten the country into it in the first place. I use the Archigos data (Goemans, Gleditsch, and Chiozza, 2009), cross-referenced with Lentz (1994), to record the leader in power for each month of the intervention; if a leadership transition occurs within a particular conflict-month, I record the identity of the replacement leader rather than his or her predecessor. I then use Lentz (1994) to note whether, for any given conflict month, the leader in power is either the leader who initiated the intervention, or is a member of the same political party.

As alternative operationalizations for supplementary analyses, I also simply note whether the leader in power is the leader who initiated the intervention, and sum the number of previous interventions carried out by the leader, under the assumption that leaders who intervene frequently will be more concerned with the reputational consequences of withdrawing short of victory. This measure follows the logic of Walter's (2006) study of why governments choose to fight some secessionist movements and not others: leaders that frequently engage in international interventions bear larger reputational costs of withdrawal short of victory, since it is likely to undermine their credibility in interventions in the future.

\section{Dispositional VARIABLES}

In the lab and survey experiments, I focus on four dispositional sources of resolve: time preferences, risk preferences, honor orientations, and to a lesser extent, trait self-control. There is an obvious levels-of-analysis issue here with regard to dispositional measures (are these the dispositions of individual leaders or the public at large?), such that I investigate some of these dispositions for both publics in general, and at the leader level, as part of growing interest in the study of individual leaders in international politics (Bueno de Mesquita and Siverson, 1995; Byman and Pollack, 2001; Stanley, 2009; Bak and Palmer, 2010; Chiozza and Goemans, 2011; Saunders, 2011; Jervis, 2013), especially by political psychologists (Feldman and Valenty, 2001; Hermann et al., 2001; Dyson, 2007; Renshon, 2008, 2009). 


\section{Time preferences}

In an ideal world, I would measure leaders' time preferences by presenting leaders with the delayed choice tasks used in the lab and survey experiment, to obtain precise estimates of leaders' $\delta$ and $\beta$ parameters. Since this is obviously not possible here, I adopt a less direct approach with two measures of leaders' time horizons. First, I calculate the average number of leadership transitions during the previous 20 years, since leaders in countries with higher turnover rates have shorter time horizons, leaving them more sensitive to short-term costs of war than leaders who can expect to remain in power for decades. Given work on the survival of leaders who take their countries to war (Bueno de Mesquita and Siverson, 1995; Chiozza and Goemans, 2004), regime type is an alternative way of looking at leaders' time horizons, but given the sample of countries considered here (the US, UK, France, China, and Russia), leadership tenure is a better and more direct measure. Second, I also operationalize leaders' time horizons by generating predicted values of leaders' survival rates from Chiozza and Goemans's (2004) leader survival model. ${ }^{10}$ The logic here is the same: leaders whose expected probability of remaining in office at any given time is low will have shorter time horizons than leaders who can expect to remain in power for some time. ${ }^{11}$

\section{Risk preferences}

I measure risk preferences using a similarly indirect approach. At the leader level, I follow Horowitz and Stam (2012), whose work on individual leader characteristics studies risk acceptance by examining leaders' military experience - the logic being that leaders who have previous military service will thus be more acceptant of the

\footnotetext{
${ }^{10}$ There are other models of leaders' survival rates - e.g. Young's (2008) - but the Chiozza and Goemans data have fewer missing values and thus are better suited to my purposes. One potential downside to the Chiozza and Goemans model is that Keele (2010) finds that many of the proportional hazards violations in the model are in fact due to unmodeled nonlinearity. Since splines are not well-suited to predictive models, I simply replicate the original model rather than attempt to modify it.

${ }^{11}$ Although the two measures of time horizons tap into the same construct, they are not highly collinear $(r=0.12)$.
} 
risks of war. ${ }^{12}$ Thus, using Lentz (1994), I generate a dichotomous variable indicating whether each leader had previous military experience before assuming power; although Horowitz and Stam (2012) distinguish between leaders whose military experience includes combat experience and those who do not, due to the far smaller number of leaders I am investigating ( $\mathrm{N}=53)$, I simply record whether leaders had any military experience, whether as part of guerilla warfare (e.g. Andropov) or as a high-level military commander (e.g. De Gaulle).

For risk preferences at the societal level, I take advantage of data from the World Values Survey (World Values Survey Association, 2009), which has two self-report items measuring respondents' risk orientations. The first item, E045, asks participants to choose between endorsing 'One should be cautious about major changes in life" vs "One should act boldly to achieve"; the second, A195, uses an item from the Schwartz Portrait Value Questionnaire (PVQ), asking "It is important to this person: being adventure and taking risks."13 Since the central premise of cross-cultural psychology is that these basic values are relatively stable in societies across time, I average across each countries' score for each item to produce a risk orientation score I use for the entire 1945-2003 time period, a maneuver that therefore treats societal risk attitudes as equivalent to a country-level fixed effect - albeit stemming from a theoretical variable (Green, Kim, and Yoon, 2001). Similarly, in their study comparing risk attitudes in China and the United States, Hsee and Weber (1999) suggest that risk acceptance increases with collectivism: societies with close social networks have higher levels of risk acceptance out of the belief that your social network will cushion you if you fall. There is no obvious analogue to this cushion in a foreign policy context (we call the international system a self-help system for a reason), but if risk orientations are general dispositions rather than highly context specific, it is worth including a measure of collectivism as well. In the World

\footnotetext{
${ }^{12}$ This argument leads to a similar conclusion - albeit through a different causal mechanism - as Sechser (2004). For a contrary perspective, see Huntington (1957); Feaver and Gelpi (2005).

${ }^{13}$ E045 was administered in China in 1990 and 1995, the US in 1990 and 1995, France in 1990, Great Britain in 1990, and Russia in 1990, 1995 and 1999. A195 was administered in China in 2007; the US in 2006, France in 2006, Great Britain in 2006, and Russia in 2006.
} 
Values Survey, collectivism is typically measured with a series of "Important child qualities" questions, in which participants are presented with "a list of qualities that children can be encouraged to learn at home", and asked to provide up to five that are especially important. I therefore calculate collectivism scores based on responses to the qualities "tolerance and respect for other people" (A035), "religious faith" (A040), "unselfishness" (A041), and "obedience" (A042). ${ }^{14}$ As before, I average across each countries' responses to produce a country-level collectivism score I use for the entire time period under investigation. ${ }^{15}$

\section{Cultures of Honor}

Honor culture is historically associated with conservative political ideology, and in the lab experiment results, conservatives score much higher in honor orientation than liberals. Thus at the leader level, I study honor culture indirectly by proxying it with political ideology, using a dummy variable indicating whether a leader is a member of a right-wing political party or not. To code ideology, I rely on Castles and Mair (1984), Lentz (1994) and Döring and Manow (2012), deriving the ideology score from the political party the leader is a member of, rather than via the leader herself. The meaning of left- and right-wing varies cross-culturally — particularly in the cases of China and Russia - so to avoid subjective coding decisions with regard to the ideological background of individual leaders, I simply code the Soviet and Chinese communist party leaders as being on the left; I also drop all of the Chinese and Russian interventions and replicate the analyses on just the democratic

\footnotetext{
${ }^{14}$ A035 was asked in China in 1990, 1995, 2001; France in 1981, 1990, 1999; Great Britain in 1981, 1990, 1998, 1999; Russia in 1990, 1995, 1999; the USA in 1982, 1990, 1995, 1999. A040 was asked in China in 1990, 1995; France in 1981, 1990, 1999; Great Britain in 1981, 1990, 1998, 1999; Russia in 1990, 1995, 1999; the USA in 1982, 1990, 1995, 1999. A041 was asked in China in 1990, 1995, 2001; France in 1981, 1990, 1999; Great Britain: 1981, 1990, 1999; Russia in 1990, 1995, 1999; the USA in 1990, 1995, 1999. A042 was asked in China in 1990, 1995, 2001; France in 1981, 1990, 1999; Great Britain in 1981, 1990, 1998, 1999; Russia in 1990, 1995, 1999; the USA in 1982, 1990, 1995, 1999.

${ }^{15}$ Whereas the experimental results pointed to nonlinear measures of risk attitudes, both of the operationalizations here are linear by necessity: the proxy for leader risk-attitudes is dichotomous, whereas the societal-level risk measures are constant for each great power through time, so there are too few observations here to meaningfully detect nonlinear effects.
} 
countries in the sample.

\section{TRAIT SELF-CONTROL}

Finally, I measure trait self-control at the societal level using two "important child qualities" items from the World Values Survey, which evaluate the degree to which respondents emphasize "self-control" (A037) and "determination and perseverence" (A039) as especially important qualities for children to have. ${ }^{16}$ As with the other WVS data, I average across each countries' responses to produce a countrylevel score I use for the entire time period.

\subsection{Results}

\section{Model selection}

Table 5.1: Model combinations

\begin{tabular}{c|cccc}
\hline & \multicolumn{4}{|c}{ Dispositional } \\
\hline \multirow{3}{*}{ Situational } & Country-level & Leader-level & Both \\
& Country-level & $\times$ & $\checkmark$ & $\checkmark$ \\
& Leader-level & $\times$ & $\times$ & $\times$ \\
& Both & $\times$ & $\checkmark$ & $\checkmark$ \\
\hline
\end{tabular}

Checkmarks indicate models ultimately selected and estimated in Table 5.2.

Since I am interested in two kinds of variables (dispositional and situational), each of which can exist at two different levels of analysis (the country/societallevel, and the leader-level), I estimate nine different sets of Boolean logit models (illustrated in Table 5.1), based upon whether the dispositional and situational variables of interest are at the country-level, the leader-level, or both. The subsequent model selection procedure proceeds as follows.

\footnotetext{
${ }^{16}$ A037 was administered in France in 1981, Great Britain in 1981, and the US in 1982. A039 was administered in China in 1990, 1995, 2001 and 2006; in France in 1981, 1990, 1999 and 2006; in Great Britain in 1981, 1990, 1998, 1999 and 2006; in the US in 1982, 1990, 1995, 1999 and 2006; and in Russia in 1990, 1995, 1999, and 2006.
} 
First, to test for the appropriate model specification, I produced eight versions of each of the nine models from Table 5.1, based on whether:

1. Duration dependence is modeled using either a logarithmic or a quadratic functional form. ${ }^{17}$

2. Unit heterogeneity is either left unmodeled, or modeled using country-fixed effects.

3. The model is estimated either using a local nonlinear minimization optimizer (nlminb) or a Broyden-Fletcher-Goldfarb Shanno (BFGS) optimizer in R. ${ }^{18}$

I then estimated these models, discarding those that fail to converge (or produce missing or nonsensical coefficient estimates). ${ }^{19}$ Importantly, none of the models with country-level fixed effects converged, such that none are included in any of the analyses reported below. I then compared coefficient estimates between the estimates produced via BFGS and those produced by nlminb, discarding those models that produced different estimates with different optimizers.

Subsequently, I used BIC scores to adjudicate between the two different functional forms of duration dependence, finding that in general, models where duration dependence is modeled with a logarithmic functional form fit better than equivalent models with a quadratic functional form of time. Of the remaining seven models, BIC scores were then used to adjudicate goodness of fit; the four best-fitting models are depicted with checkmarks in Table 5.1. ${ }^{20}$ To double-check

\footnotetext{
${ }^{17}$ Carter and Signorino (2010) recommend modeling duration dependence with a cubic polynomial, but models with cubic polynomials either failed to converge, or fit the data poorly, as indicated by Akaike Information Criterion (AIC) and Bayesian Information Criterion (BIC) scores. Keele (2008) discusses the use of nonparametric splines to model duration dependence, but given the computationally intense analyses that follow, I stick to the simpler logarithmic and quadratic functional forms mentioned above.

${ }^{18}$ These optimizers are selected because of their relative speed; as discussed below, genetic optimization (using genoud (Mebane and Sekhon, 2011)) and simulated annealing (SANN) are employed for the final four conjunctive models to ensure the stability of the estimates.

${ }^{19}$ Importantly, all of the models that failed to estimate with one optimizer also failed to estimate with the other optimizers, increasing our confidence in the stability of the results presented below.

${ }^{20} \mathrm{~A}$ fifth model, with both country-level and leader-level situational variables, and only countrylevel dispositional variables, had the best model fit based on AIC and BIC scores, but is relatively ill-equipped at testing my theory, so I focus on the other four models.
} 
the stability of these models, I reestimated them using a genetic optimization algorithm (genoud) (Mebane and Sekhon, 2011) and a simulated annealing optimizer (SANN), finding the coefficient estimates hold. Finally, in order to take into account dependence within military interventions, I calculated $N=5000$ cluster bootstraps (Field and Welsh, 2007) to produce more accurate standard errors. Since bootstrapped Boolean estimates tend to have extremely long tails, I employ a BFGS optimizer with box-constraints (L-BFGS-B) set to $\pm 3 \sigma .^{21}$

\section{RESOLVE: SITUATIONAL AND DISPOSITIONAL}

The results for these four models are presented in Table 5.2. The first model in Table 5.2 estimates the impact of country-level situational variables and leader-level dispositional ones; the second and fourth models add leader-level situational variables, while the third and fourth models add country-level dispositional variables. The distribution of bootstrapped coefficient estimates, depicted in Figures 5.2 - 5.5, show that normal-theory $\mathrm{p}$-values would be inappropriate here, so I calculate the $\mathrm{p}$ values shown in Table 5.2 simply by estimating the percentage of bootstrapped estimates crossing zero (denoted by the grey shading in the distribution plots). Rather than discussing the results of each of the four models separately, I group them together, to better get across a sense of the robustness of the results and illuminate the relative contribution of leader- and country-level determinants of resolve to conflict outcomes.

Beginning with the situational sources of resolve, we find relatively weak support for the conventional wisdom that resolve is shaped by the costs of fighting: although higher levels of costs of fighting are negatively associated with victory (moving from the minimum to the maximum level of costs of fighting, holding all other variables at their means, is associated with a $2.43 \%-2.95 \%$ decrease in the

\footnotetext{
${ }^{21} \sigma$ is calculated based on the original (unbootstrapped) estimates. In this sense, optimization with box constraints is similar in spirit to a penalized likelihood, in that it uses prior information to constrain the shape of the likelihood function. In general, the inclusion of the box constraints brings in the tails and lowers the coefficient estimates for the bootstrapped distributions, but does not affect the statistical significance of the estimates.
} 
Table 5.2: Boolean model estimates: situation $x$ disposition

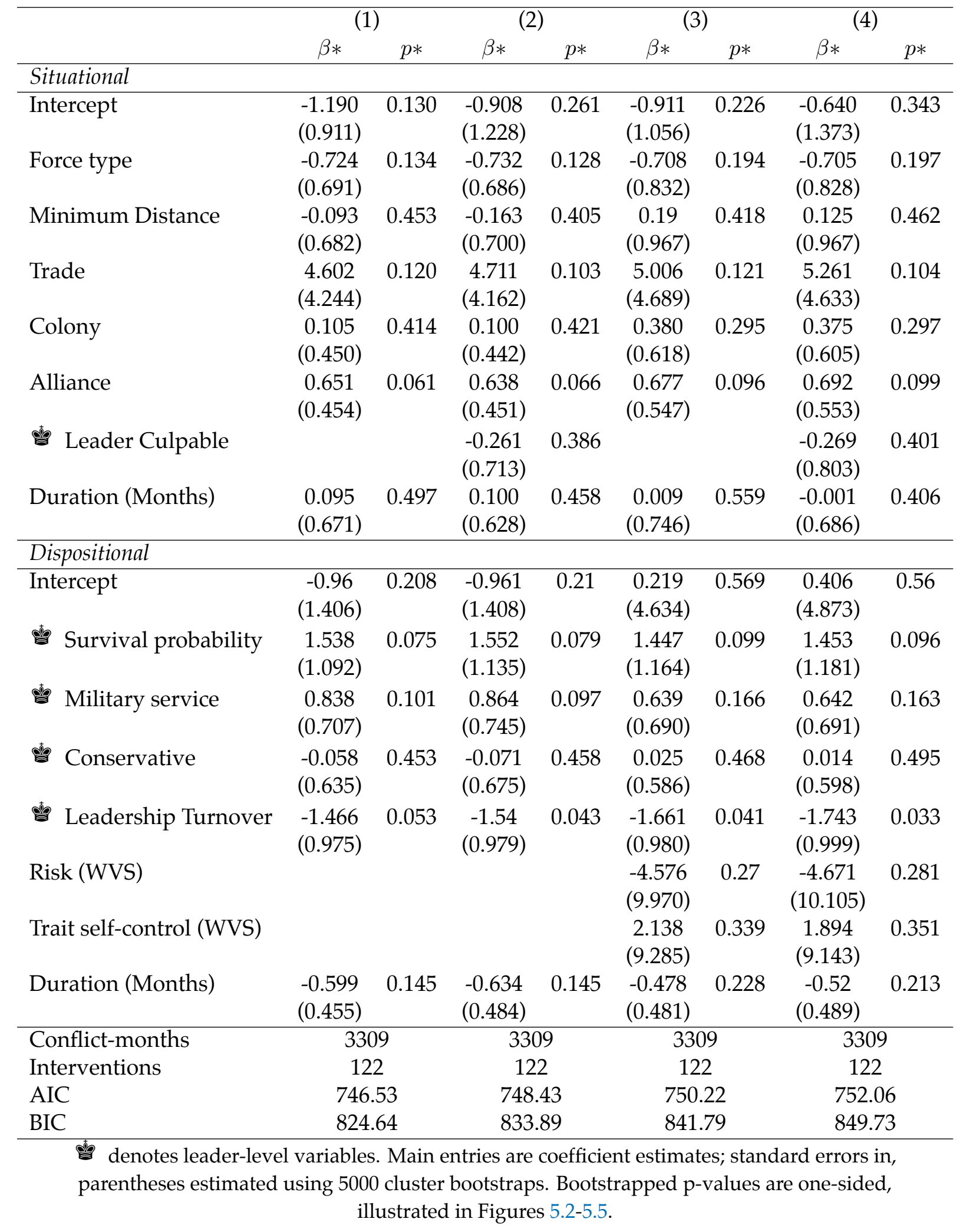




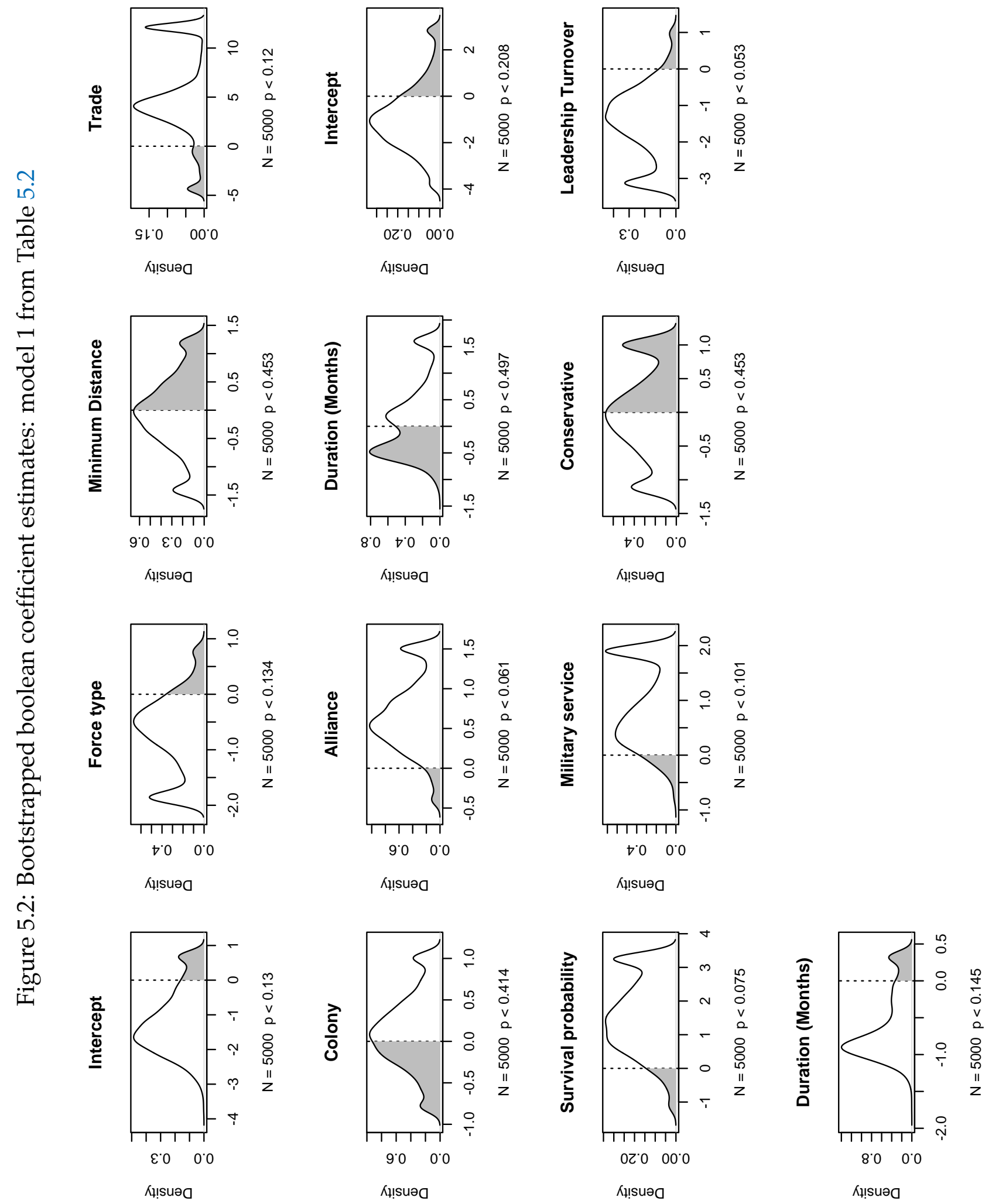




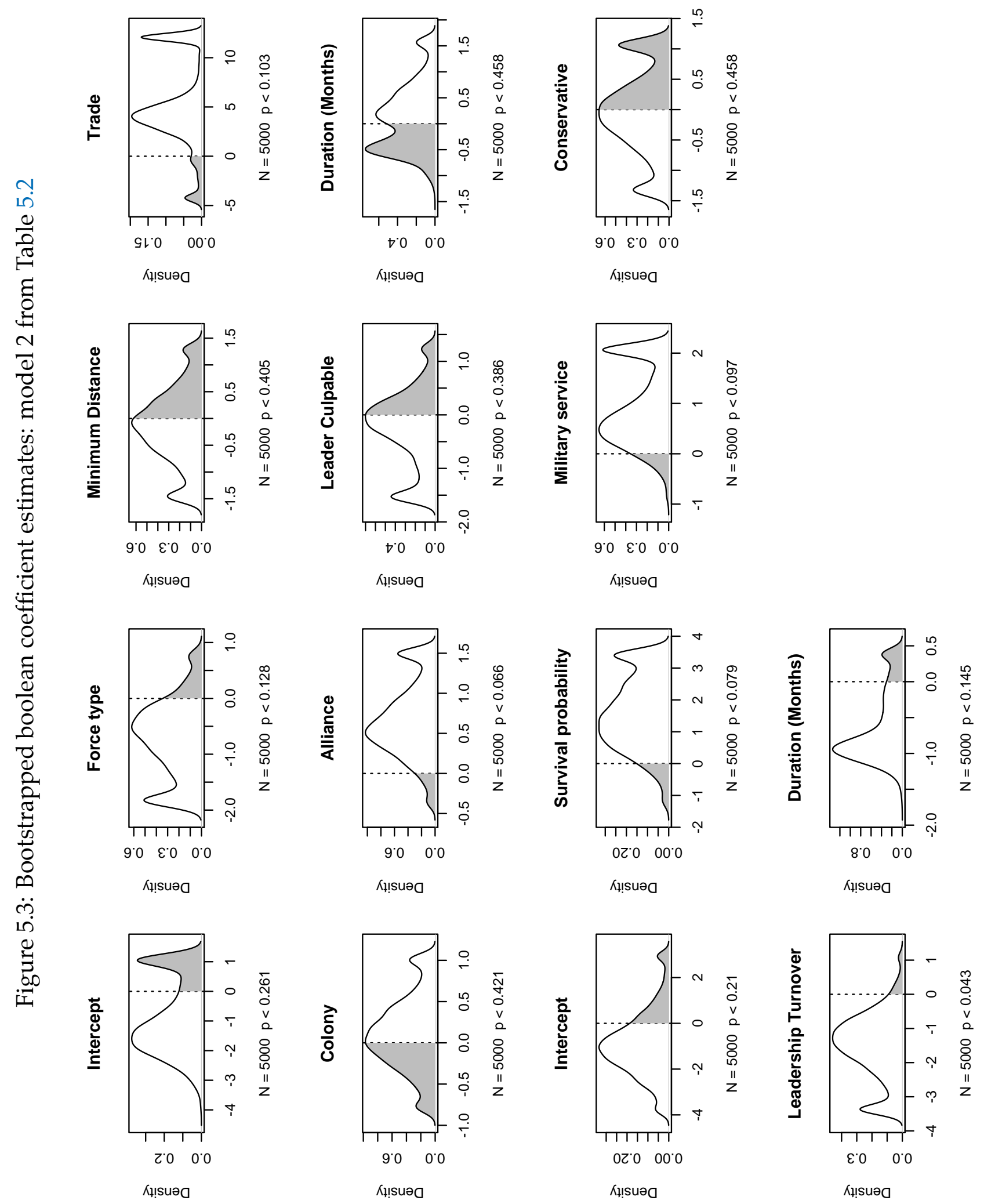




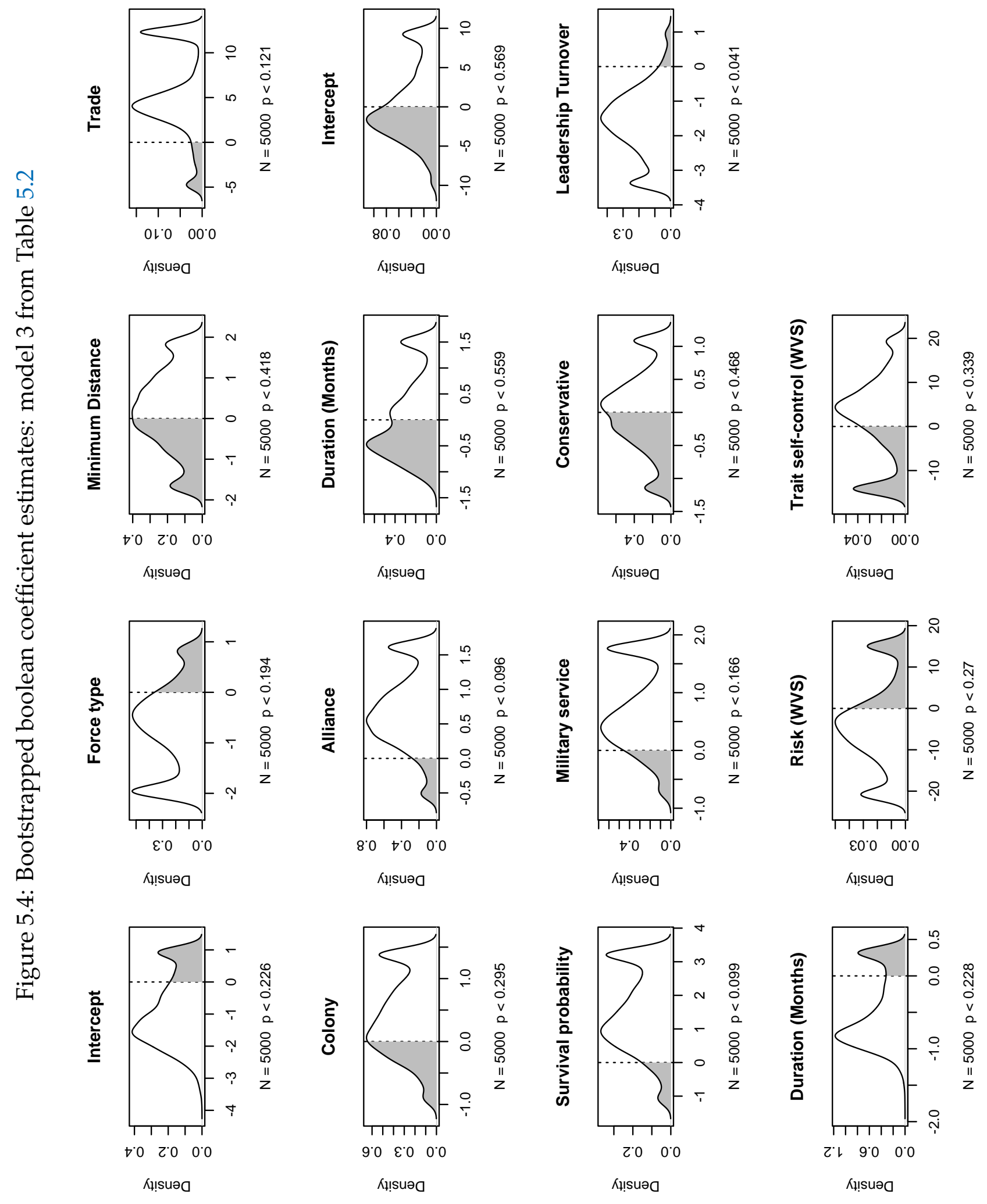



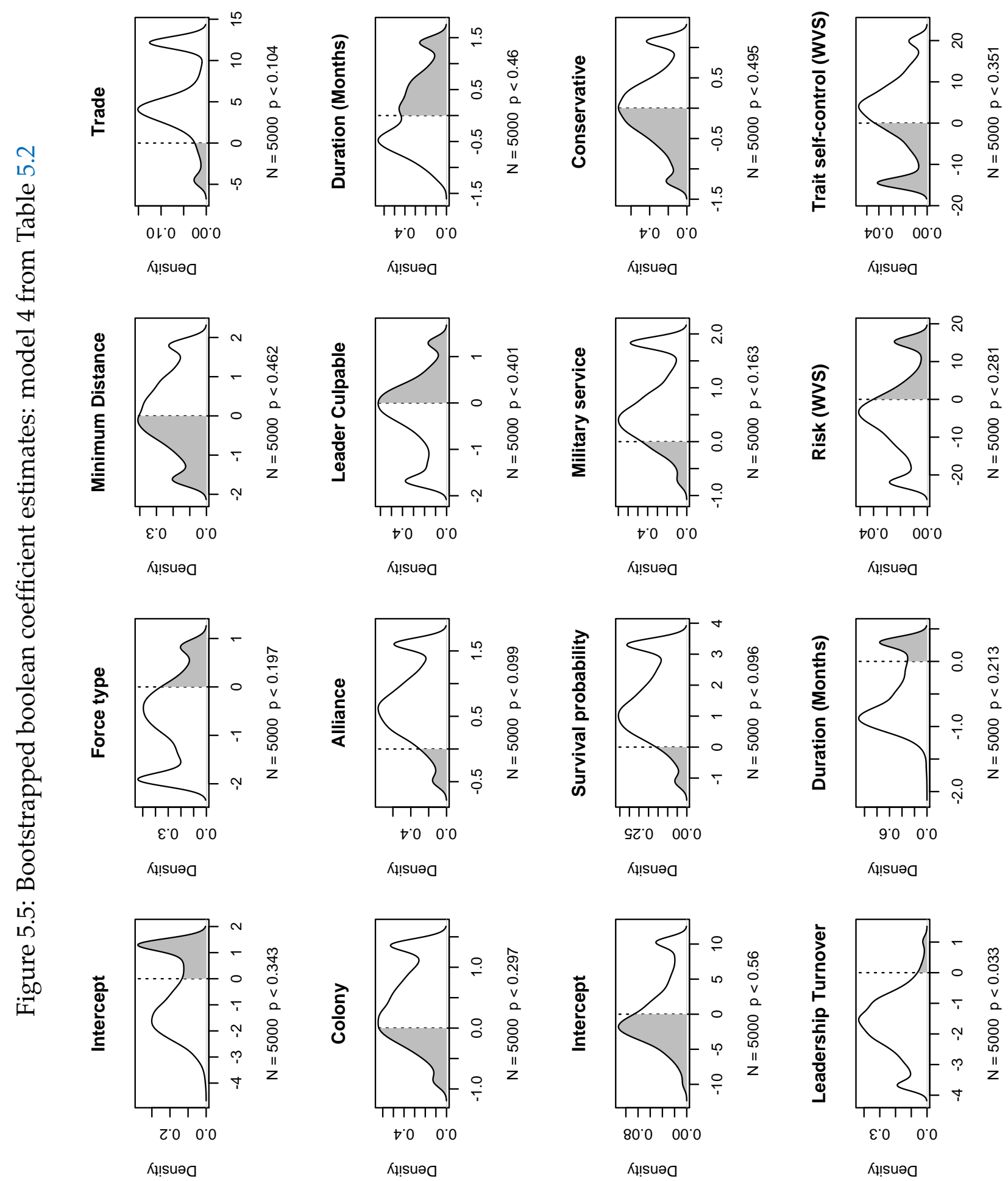
probability of victory, depending on the model specification), it is significant only at the $p<0.128$ to $p<0.197$ level, failing to meet conventional standards of statistical significance.

We find stronger evidence in support of some of the costs of backing down. Although minimum distance from the homeland - a measure of the gravity of the issues at stake - is not significantly related to the probability of victory, great powers with higher volumes of trade with the state in which the intervention is taking place are more likely to prevail; moving from the minimum to the maximum level of trade is associated with a $12.19 \%-17.29 \%$ increase in the probability of victory, with associated $\mathrm{p}$-values ranging from $p<0.103$ to $p<0.121$. Trade flows are likely a better test of stakes in great power military interventions than geographic distance, since by definition one of the things that makes great power great is their ability to project their power over distances, such that distance may be a more appropriate measure of stakes for lesser powers than for great ones. ${ }^{22}$ Indeed, recent survey data shows that by a wide margins, Americans are more likely to perceive interests at stake in the Middle East and Northeast Asia than Latin America. ${ }^{23}$ Whether the intervention takes place in a current or former colony has no significant effect on the probability of victory, unlike if it takes place in an ally: since great powers who are seen as abandoning an ally pay stronger reputational costs, interventions in allied states are associated with a $2.36 \%-2.61 \%$ higher probability of victory $(p<0.061$ to $p<0.099) .{ }^{24}$

In this sense, higher costs of backing down - both in terms of intrinsic issues at stake (such as trade) and reputation costs (such as the specter of abandoning an ally) - appear to increase the odds of victory. However, our measure of leaderlevel reputation costs - whether the leader or a fellow member of the leader's party initiated the intervention - never achieves anything close to statistical significance. Thus, unlike Croco (2011), I find no evidence that culpable leaders are more likely to

\footnotetext{
${ }^{22}$ I am grateful to Patrick Shea for helpful discussions on this point.

${ }^{23}$ I am grateful to Rick Herrmann for sharing his survey data.

${ }^{24}$ For a discussion contextualizing these effect sizes, see the conclusion.
} 
achieve better war outcomes. The situational analyses suggest that different types of costs of war indeed positively predict victory in military interventions, but the relevant costs of war appear to be at the country-level rather than the leader-level; I probe the robustness of the result in alternative model specifications in the next section.

Turning to the dispositional analyses, both measures of leaders' time horizons - the average number of leaders in power over the past twenty years (since leaders whose predecessors remained in office for relatively short periods of time should have shorter time horizons), and leaders' own probability of remaining in office significantly affect the probability of victory. Moving from the lowest to the highest rate of leadership turnover is associated with a $3.54 \%-4.48 \%$ decrease in the probability of victory ( $p<0.033$ to $p<0.053$ ). Meanwhile, moving from the lowest to highest survival probability is associated with a $3.56 \%-4.05 \%$ increase in the probability of victory ( $p<0.075$ to $p<0.099$ ). Therefore, for both measures, longer leader time horizons predict a greater likelihood of victory. We find both substantively and statistically weaker results for risk preferences, but in the anticipated direction: leaders with histories of military service (and thus, greater risk acceptance) are $1.67 \%$ to $2.05 \%$ more likely to achieve victory ( $p<0.097$ to $p<0.166$ ). However, we find no significant results with our measure of honor culture: leaders from conservative political parties are no more likely to achieve victory than leaders from liberal parties - a null effect that replicates even when we drop the non-democratic great powers from the sample, as discussed below. Importantly, neither of the societal-level dispositional variables - risk attitudes and trait-selfcontrol - significantly predict the probability of victory.

Thus, our dispositional sources of resolve display the opposite dynamic as our situational measures: whereas military outcomes appear to be shaped by countrylevel situational variables but not leader-level ones, they also appear to be shaped by leader-level dispositional variables, but not country-level ones. Indeed, AIC and BIC scores suggest that the best fitting model of the four models presented in 
Table 5.2 is model 1, which restricts itself to country-level situational variables and leader-level dispositional ones. It is possible that these results are simply the artifact of the particular measures employed - the analyses use more country-level situational variables than leader-level ones, and many of the leader-level dispositional variables are completely time-varying, whereas the country-level dispositional variables are constant. Nonetheless, as discussed towards the end of this chapter, these results are still instructive inasmuch as they suggest that we should understand dispositional characteristics in IR as being fluid rather than fixed. Similarly, it is possible that the lack of significant societal-level dispositional characteristics is due to the relatively small sample of countries considered here, but it remains telling that the behavior of the five great powers that have dominated much of the international agenda in the post-WWII era is better explained by dispositional variables of their leaders than of the countries as a whole.

Zooming out from the individual coefficients to the overall model dynamics, Figure 5.6 plots the fitted values for the dispositional and situational measures of resolve against the probability of victory, calculating the fitted values based on the coefficient estimates for model 1 of Table 5.2. The wireframe plot illustrates the joint impact of situational and dispositional sources of resolve. As the situational resolve scores (on the z-axis) increase, so too does the predicted probability of victory; as the dispositional resolve scores (on the $\mathrm{x}$-axis) increase, the predicted probability of victory increases as well. The curvature of the plot surface shows the extent to which dispositional aspects of resolve moderate the impact of situational features: the probability of victory is highest when both situational and dispositional resolve are high; when dispositional resolve is high but situational resolve is low (the frontleft vertex in Figure 5.6), the probability of victory is relatively low, as is the case when situational resolve is high but dispositional resolve is low (the back-right vertex). 
Table 5.3: Situation $x$ disposition alternative model specifications)

\begin{tabular}{|c|c|c|c|c|c|c|c|c|c|c|}
\hline & \multicolumn{2}{|c|}{ (1) } & \multicolumn{2}{|c|}{ (2) } & \multicolumn{2}{|c|}{ (3) } & \multicolumn{2}{|c|}{ (4) } & \multicolumn{2}{|c|}{ (5) } \\
\hline & $\beta *$ & $p *$ & $\beta *$ & $p *$ & $\beta *$ & $p *$ & $\beta *$ & $p *$ & $\beta *$ & $p *$ \\
\hline \multicolumn{11}{|l|}{ Situational } \\
\hline Intercept & $\begin{array}{c}-1.185 \\
(1.130)\end{array}$ & 0.177 & $\begin{array}{c}-0.966 \\
(1.420)\end{array}$ & 0.292 & $\begin{array}{c}-1.91 \\
(1.101)\end{array}$ & 0.077 & $\begin{array}{c}-0.663 \\
(1.159)\end{array}$ & 0.258 & $\begin{array}{c}-0.918 \\
(1.155)\end{array}$ & 0.274 \\
\hline Force type & $\begin{array}{c}-0.328 \\
(0.906)\end{array}$ & 0.369 & $\begin{array}{c}-0.306 \\
(0.899)\end{array}$ & 0.38 & $\begin{array}{c}-0.604 \\
(0.793)\end{array}$ & 0.217 & $\begin{array}{c}-0.931 \\
(0.752)\end{array}$ & 0.083 & $\begin{array}{c}-0.714 \\
(0.820)\end{array}$ & 0.188 \\
\hline Minimum Distance & $\begin{array}{c}0.408 \\
(1.119)\end{array}$ & 0.354 & $\begin{array}{c}0.312 \\
(1.135)\end{array}$ & 0.399 & $\begin{array}{c}0.499 \\
(1.042)\end{array}$ & 0.332 & $\begin{array}{l}-0.341 \\
(0.762)\end{array}$ & 0.329 & $\begin{array}{c}0.164 \\
(1.077)\end{array}$ & 0.435 \\
\hline Trade & $\begin{array}{c}7.253 \\
(5.490)\end{array}$ & 0.084 & $\begin{array}{c}7.485 \\
(5.335)\end{array}$ & 0.070 & $\begin{array}{c}5.564 \\
(4.983)\end{array}$ & 0.100 & $\begin{array}{c}5.107 \\
(4.700)\end{array}$ & 0.114 & $\begin{array}{c}5.243 \\
(4.831)\end{array}$ & 0.111 \\
\hline Colony & $\begin{array}{c}0.556 \\
(0.831)\end{array}$ & 0.26 & $\begin{array}{c}0.569 \\
(0.827)\end{array}$ & 0.266 & $\begin{array}{c}0.538 \\
(0.704)\end{array}$ & 0.253 & $\begin{array}{c}0.153 \\
(0.470)\end{array}$ & 0.369 & $\begin{array}{c}0.287 \\
(0.619)\end{array}$ & 0.342 \\
\hline Alliance & $\begin{array}{c}1.261 \\
(0.746)\end{array}$ & 0.037 & $\begin{array}{c}1.24 \\
(0.755)\end{array}$ & 0.049 & $\begin{array}{c}0.752 \\
(0.523)\end{array}$ & 0.067 & $\begin{array}{c}0.564 \\
(0.465)\end{array}$ & 0.099 & $\begin{array}{c}0.671 \\
(0.552)\end{array}$ & 0.104 \\
\hline 畨 Leader Culpable & & & $\begin{array}{l}-0.207 \\
(0.755)\end{array}$ & 0.402 & & & & & $\begin{array}{c}-0.033 \\
(0.731)\end{array}$ & 0.419 \\
\hline 当 Leader Initiate & & & & & $\begin{array}{c}0.954 \\
(0.768)\end{array}$ & 0.069 & & & & \\
\hline Prev. Leader Int. & & & & & & & $\begin{array}{c}-1.59 \\
(1.083)\end{array}$ & 0.037 & & \\
\hline Duration (Months) & $\begin{array}{l}-0.119 \\
(0.811)\end{array}$ & 0.385 & $\begin{array}{c}-0.123 \\
(0.770)\end{array}$ & 0.399 & $\begin{array}{l}-0.107 \\
(0.597)\end{array}$ & 0.337 & $\begin{array}{c}0.239 \\
(0.663)\end{array}$ & 0.408 & $\begin{array}{c}0.045 \\
(0.772)\end{array}$ & 0.527 \\
\hline \multicolumn{11}{|l|}{ Dispositional } \\
\hline Intercept & $\begin{array}{l}-4.268 \\
(3.239)\end{array}$ & 0.088 & $\begin{array}{l}-4.167 \\
(3.178)\end{array}$ & 0.092 & $\begin{array}{l}-0.466 \\
(4.686)\end{array}$ & 0.409 & $\begin{array}{l}-0.787 \\
(1.460)\end{array}$ & 0.24 & $\begin{array}{c}-0.33 \\
(4.677)\end{array}$ & 0.401 \\
\hline Survival probability & $\begin{array}{c}4.542 \\
(3.010)\end{array}$ & 0.059 & $\begin{array}{c}4.472 \\
(2.914)\end{array}$ & 0.056 & $\begin{array}{c}1.479 \\
(1.188)\end{array}$ & 0.100 & $\begin{array}{c}1.418 \\
(1.149)\end{array}$ & 0.108 & $\begin{array}{c}1.622 \\
(1.180)\end{array}$ & 0.081 \\
\hline 当 Military service & $\begin{array}{c}0.336 \\
(0.677)\end{array}$ & 0.31 & $\begin{array}{c}0.328 \\
(0.664)\end{array}$ & 0.317 & $\begin{array}{c}0.544 \\
(0.774)\end{array}$ & 0.245 & $\begin{array}{c}0.822 \\
(0.680)\end{array}$ & 0.092 & $\begin{array}{c}0.573 \\
(0.689)\end{array}$ & 0.185 \\
\hline 尚 Conservative & $\begin{array}{c}0.066 \\
(0.626)\end{array}$ & 0.434 & $\begin{array}{c}0.057 \\
(0.623)\end{array}$ & 0.446 & $\begin{array}{c}0.136 \\
(0.635)\end{array}$ & 0.414 & $\begin{array}{c}-0.207 \\
(0.649)\end{array}$ & 0.382 & $\begin{array}{c}0.03 \\
(0.589)\end{array}$ & 0.491 \\
\hline 畨 Leadership Turnover & $\begin{array}{c}-1.19 \\
(1.027)\end{array}$ & 0.111 & $\begin{array}{l}-1.265 \\
(1.011)\end{array}$ & 0.092 & $\begin{array}{l}-1.491 \\
(1.121)\end{array}$ & 0.081 & $\begin{array}{c}-1.42 \\
(0.932)\end{array}$ & 0.054 & $\begin{array}{l}-1.565 \\
(1.029)\end{array}$ & 0.056 \\
\hline Risk (WVS) & & & & & $\begin{array}{c}-4.186 \\
(11.407)\end{array}$ & 0.304 & & & & \\
\hline Trait self-control (WVS) & & & & & $\begin{array}{c}2.857 \\
(10.275)\end{array}$ & 0.325 & & & $\begin{array}{c}0.781 \\
(9.524)\end{array}$ & 0.418 \\
\hline Collectivism (WVS) & & & & & & & & & $\begin{array}{l}-3.137 \\
(7.638)\end{array}$ & 0.32 \\
\hline Duration (Months) & $\begin{array}{c}-0.403 \\
(0.541)\end{array}$ & 0.268 & $\begin{array}{c}-0.443 \\
(0.554)\end{array}$ & 0.251 & $\begin{array}{c}-0.243 \\
(0.572)\end{array}$ & 0.283 & $\begin{array}{l}-0.687 \\
(0.386)\end{array}$ & 0.103 & $\begin{array}{l}-0.475 \\
(0.585)\end{array}$ & 0.212 \\
\hline Conflict-months & \multicolumn{2}{|c|}{2671} & \multirow{2}{*}{\multicolumn{2}{|c|}{2671}} & \multicolumn{2}{|c|}{3309} & \multicolumn{2}{|c|}{3309} & \multicolumn{2}{|c|}{3309} \\
\hline Interver & \multirow{2}{*}{\multicolumn{2}{|c|}{$\begin{array}{l}88 \\
Y e s\end{array}$}} & & & \multirow{2}{*}{\multicolumn{2}{|c|}{122}} & 12 & & & \\
\hline Democracies only? & & & \multicolumn{2}{|c|}{$y^{60}$} & & & \multicolumn{2}{|c|}{$\mathrm{No}$} & \multicolumn{2}{|c|}{ No } \\
\hline AIC & \multicolumn{2}{|c|}{579.03} & \multicolumn{2}{|c|}{580.95} & \multicolumn{2}{|c|}{750.18} & \multicolumn{2}{|c|}{744.56} & \multicolumn{2}{|c|}{752.23} \\
\hline BIC & \multicolumn{2}{|c|}{655.59} & \multicolumn{2}{|c|}{663.40} & \multicolumn{2}{|c|}{847.85} & \multicolumn{2}{|c|}{830.02} & \multicolumn{2}{|c|}{849.90} \\
\hline
\end{tabular}

denotes leader-level variables. Main entries are coefficient estimates; standard errors in parentheses, estimated using 5000 cluster bootstraps. Bootstrapped p-values are one-sided, illustrated in Figures D.1-D.5 in Appendix D. 
Figure 5.6: Fitted values: conjunctural causation

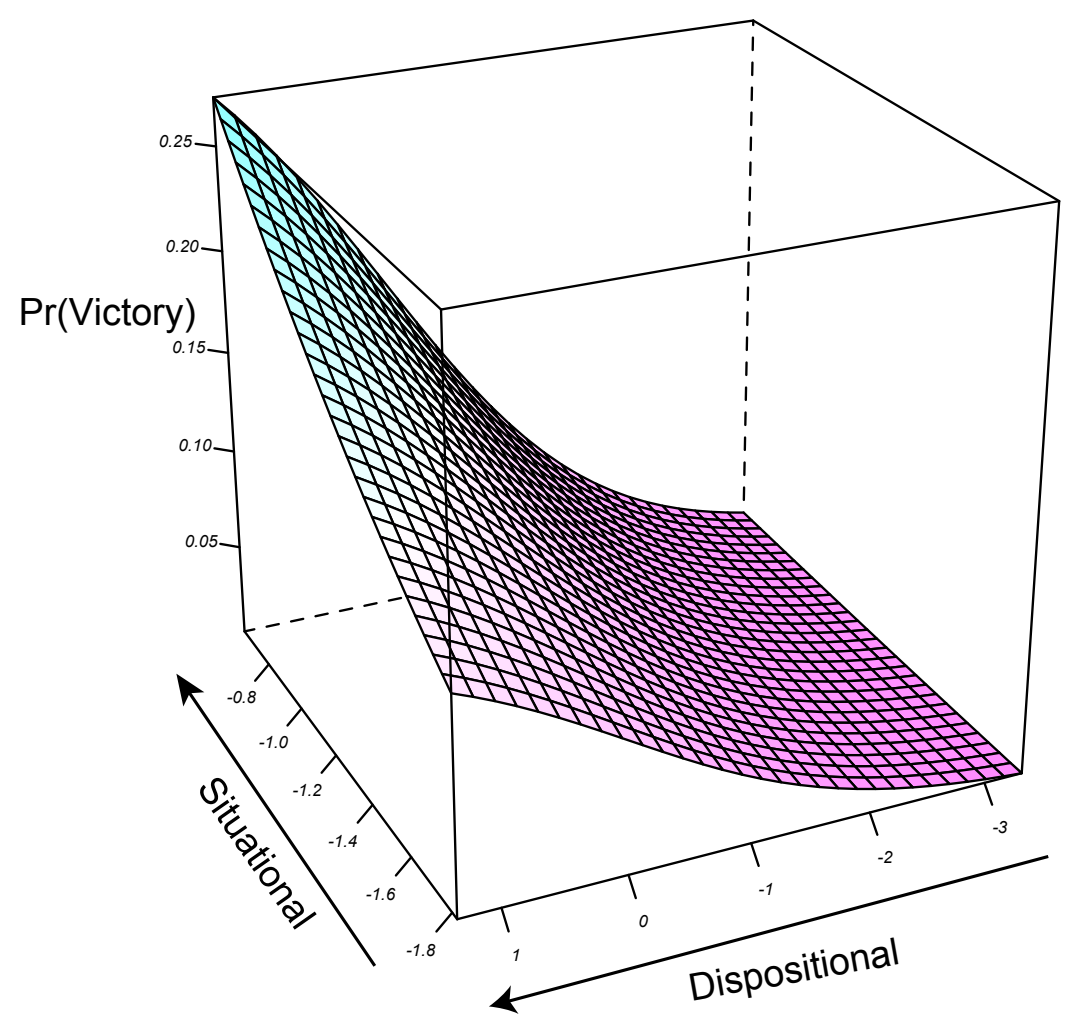

The fitted values of the dispositional and situational resolve scores show that resolve indeed boosts the probability of victory: as actors' dispositional resolve increases, so too does their likelihood of attaining their primary mission objective; the same can be said for increases in their situational resolve scores. Since victory here is modeled as a function of both situational and dispositional resolve, the probability of victory is highest when both situational and dispositional resolve scores are high (the top-left-hand vertex of the plot), and lowest when both situational and dispositional resolve scores are low (the bottom-right-hand vertex). The fitted value scores here are derived from Model 1 in Table 5.2 and are clipped at the 2nd and 98th percentiles to drop outliers.

\section{Alternative model specifications}

To probe the robustness of the results, models 1 and 2 in Table 5.3 replicate the two best-fitting models from Table 5.2, but only for those military interventions carried out by the United States, France, and the United Kingdom, the three democratic great powers in the data. ${ }^{25}$ The basic pattern of results remains the same, with two

${ }^{25}$ The lower AIC and BIC suggest there may be heterogeneity across regime type in that the model fit appears to be superior amongst the democratic countries than in the pooled model with both democracies and non-democracies, but if this were the case we would expect the democratic model to correctly predict a greater proportion of its observations than the pooled model; instead, ePCP scores (Herron, 1999) suggest the pooled model has higher predictive power than the demo- 
interesting exceptions. First, whereas force type approached statistical significance in some of the models in Table 5.2, both its substantive and its statistical impact shrinks here: amongst democratic countries, an increase in the cost of fighting is associated with only a $0.6 \%-0.8 \%$ drop in the probability of victory, far from statistical significance $(p<0.369$ to $p<0.380)$. A comparison of the distributions of the two sets of bootstrapped coefficients shows we cannot claim that the substantive impact of the type of force is in fact significantly stronger in the pooled model than in the democratic subsample ( $p<0.279$ to $p<0.294$ ), but given conventional wisdom about democratic publics being especially sensitive to the costs of fighting, the absence of a statistically significant relationship between the cost of fighting and the probability of democratic powers emerging victorious is nonetheless noteworthy. ${ }^{26}$ Second, although the effect of previous military service is significant in the full sample, among the democratic subsample it is associated with only a $0.7 \%$ increase in the probability of victory, which fails to attain statistical significance ( $p<0.310$ to $p<0.317$ ). As with the case of force type, we need to be cautious about reading too much into this result, as an examination of the distribution of the bootstrapped coefficients fails to find that the impact of previous military service amongst democratic publics is significantly smaller than in the pooled model ( $p<0.176$ to $p<0.201$ ), but given that non-democratic countries in our sample are more likely to have leaders with previous military service than the democratic countries, the pattern is sensible. Importantly, the measure of leaders' honor orientations - whether they are a member of a conservative political party or not remains nonsignificant amongst the democratic countries, suggesting that its lack of significance in the full sample is not simply due to the crudeness of the measure in the context of non-democratic countries.

The next two models in Table 5.3 focus on alternative ways of operationalizing leader-level reputation costs. Model 3 in Table 5.3 replicates model 4 from Table 5.2, cratic model. I discuss predictive fit in greater detail later in this chapter.

${ }^{26}$ The p-value comes from comparing the proportion of bootstrapped coefficient estimates in the pooled model greater than the mean bootstrapped coefficient estimate in the democratic subsample. 
but using whether the leader in power also initiated the intervention as a measure of leader-level costs of backing down. Interestingly, we see that leaders who initiate conflicts themselves - and thus are likely to pay steeper reputational costs if they back down short of victory - are in fact 3.54\% more likely to prevail $(p<0.069)$. Thus, whereas Croco (2011) finds that leader culpability is a better measure than leader initiation as a measure of leaders' stakes in conflict, when it comes to great power military interventions in the postwar military era, the converse appears to be true. However, it should be noted that there is less confidence in the robustness of the result, in that model 2 from Table 5.2 failed to converge when leader initiation was used instead of leader culpability.

Model 4 in Table 5.3 replicates model 3 from Table 5.2, but using the previous number of military interventions the leader has initiated as a measure of leaderlevel reputation costs, the logic being that reputational considerations should matter more for leaders who intervene more frequently. Instead, we find that an increase from the minimum to maximum number of previous interventions corresponds with a $4.36 \%$ decrease in the probability of victory $(p<0.037)$ - an effect opposite to what the leader-level reputation cost argument would suggest. ${ }^{27}$ This negative coefficient, coupled with the fragility of the results from model 3, thereby offer further support to the findings from Table 5.2 that leader-level costs of backing down seem to play less of a role than the country-level costs of backing down.

Finally, model 5 replicates model 4 from Table 5.2, but using the World Value Survey collectivism scores as an alternative measure for societal risk attitudes. Once

\footnotetext{
${ }^{27}$ One potential explanation for this negative effect is that leaders with previous military interventions under their belt may actually bear lower reputational costs for backing down, already having established their reputations for resolve. Indeed, Wolford (2007) presents a formal model in which successors are more likely to be subject to (and initiate) military challenges than incumbents, whose reputations for resolve are already cemented. The empirical record, however, is less clear: Gelpi and Grieco (2001) find that leaders are less likely to be targeted for military crises the longer they spend in office, but Bak and Palmer (2010) finds that the effect of leadership tenure on the probability of military challenges is conditional on leaders' age - young leaders are actually less likely to be subjected to a challenge early on in their term, contrary to the leader-level reputation arguments (although they do not test whether leaders early on in their tenure are more likely to initiate military challenges themselves). Similarly, Horowitz, McDermott, and Stam (2005) find that older leaders are more likely to initiate military disputes.
} 
again, the measure is far from statistically significant, showing the weakness of time-invariant societal measures in predicting military outcomes.

\section{RESOLVE: SITUATIONAL OR DISPOSITIONAL}

The above analyses have modeled the situational and dispositional components of resolve as operating through a conjunctural causal path (Braumoeller, 2003): the probability of victory is relatively high when both situational and dispositional resolve scores are high, but relatively low when either dispositional or situational resolve scores are low. However, it is also worth testing whether these components of resolve operate using a substitutable causal path, such that the probability of victory will be relatively high if either situational or dispositional sources of resolve are high. This alternative path is worth estimating because folk theories of resolve suggest that resolve's impact on outcomes can be high even when the costs are high relative to the stakes.

Modeling this alternative boolean logic requires a different functional form that of equation 5.2 below, where $\lambda$ refers to the inverse logit function $\frac{1}{1+e^{-x}}$ :

$$
\operatorname{Pr}\left(y_{i, t}=1 \mid \alpha, \mathrm{B}, x_{i, t}\right)=1-\left(1-\lambda\left(\alpha_{1}+B_{S} x_{i, t}\right)\right) *\left(1-\lambda\left(\alpha_{2}+B_{D} x_{i, t}\right)\right)
$$

As was the case in equation 5.1, the situational determinants of resolve are grouped into the path depicted by the vector $B_{S}$, and the dispositional determinants of resolve into the path depicted by $B_{D}$, with $P_{i, t}$ as the probability that the great power achieves its objectives at time $t$. However, the revised functional form means that we can model the probability of victory as a function of either $B_{S}$ or $B_{D} \cdot{ }^{28}$

Table 5.4 estimates new version of models 1 and 3 from Table 5.2, dropping the minimum distance variable in order to allow the models to converge. ${ }^{29}$ The basic

\footnotetext{
${ }^{28}$ To understand the new functional form, recall that by DeMorgan's law, $\left.(\overline{A \cap B})=\bar{A} \cup \bar{B}\right)$. Thus, $(\overline{\bar{A} \cap \bar{B}})=A \cup B$.

${ }^{29}$ As the substitutable Boolean statistical models proved harder to estimate than their conjunc-
} 
Table 5.4: Boolean model estimates: situation or disposition

\begin{tabular}{|c|c|c|c|c|}
\hline & \multicolumn{2}{|c|}{$(1)$} & \multicolumn{2}{|c|}{ (2) } \\
\hline & $\beta *$ & $p *$ & $\beta *$ & $p *$ \\
\hline \multicolumn{5}{|l|}{ Situational } \\
\hline Intercept & $\begin{array}{l}-2.526 \\
(0.804)\end{array}$ & 0.000 & $\begin{array}{l}-2.243 \\
(0.565)\end{array}$ & 0.000 \\
\hline Force type & $\begin{array}{c}-0.769 \\
(0.595)\end{array}$ & 0.09 & $\begin{array}{l}-0.682 \\
(0.508)\end{array}$ & 0.091 \\
\hline Trade & $\begin{array}{c}4.343 \\
(4.133)\end{array}$ & 0.146 & $\begin{array}{c}4.026 \\
(3.795)\end{array}$ & 0.142 \\
\hline Colony & $\begin{array}{c}0.500 \\
(0.607)\end{array}$ & 0.208 & $\begin{array}{c}0.257 \\
(0.427)\end{array}$ & 0.279 \\
\hline Alliance & $\begin{array}{c}0.628 \\
(0.453)\end{array}$ & 0.069 & $\begin{array}{c}0.548 \\
(0.416)\end{array}$ & 0.092 \\
\hline Duration (Months) & $\begin{array}{c}-0.557 \\
(0.152)\end{array}$ & 0.000 & $\begin{array}{l}-0.559 \\
(0.122)\end{array}$ & 0.000 \\
\hline \multicolumn{5}{|l|}{ Dispositional } \\
\hline Intercept & $\begin{array}{l}-8.915 \\
(4.796)\end{array}$ & 0.001 & $\begin{array}{r}-17.510 \\
(6.360)\end{array}$ & 0.001 \\
\hline Survival probability & $\begin{array}{c}2.906 \\
(2.346)\end{array}$ & 0.091 & $\begin{array}{c}2.312 \\
(2.343)\end{array}$ & 0.171 \\
\hline 声 Military service & $\begin{array}{c}4.755 \\
(4.953)\end{array}$ & 0.14 & $\begin{array}{c}15.08 \\
(4.814)\end{array}$ & 0.007 \\
\hline 畨 Conservative & $\begin{array}{c}0.167 \\
(1.354)\end{array}$ & 0.439 & $\begin{array}{c}0.789 \\
(1.458)\end{array}$ & 0.287 \\
\hline 声 Leadership Turnover & $\begin{array}{l}-23.169 \\
(17.399)\end{array}$ & 0.012 & $\begin{array}{l}-26.635 \\
(23.213)\end{array}$ & 0.025 \\
\hline Risk (WVS) & & & $\begin{array}{c}-8.931 \\
(26.607)\end{array}$ & 0.357 \\
\hline Trait self-control (WVS) & & & $\begin{array}{c}7.764 \\
(23.957)\end{array}$ & 0.327 \\
\hline Duration (Months) & $\begin{array}{l}-0.290 \\
(0.393)\end{array}$ & 0.216 & $\begin{array}{l}-0.210 \\
(0.369)\end{array}$ & 0.304 \\
\hline Conflict-months & 330 & & 330 & \\
\hline Interventions & 12 & & 12 & \\
\hline AIC & 741. & & 745. & \\
\hline $\mathrm{BIC}$ & 815. & & 830. & \\
\hline
\end{tabular}



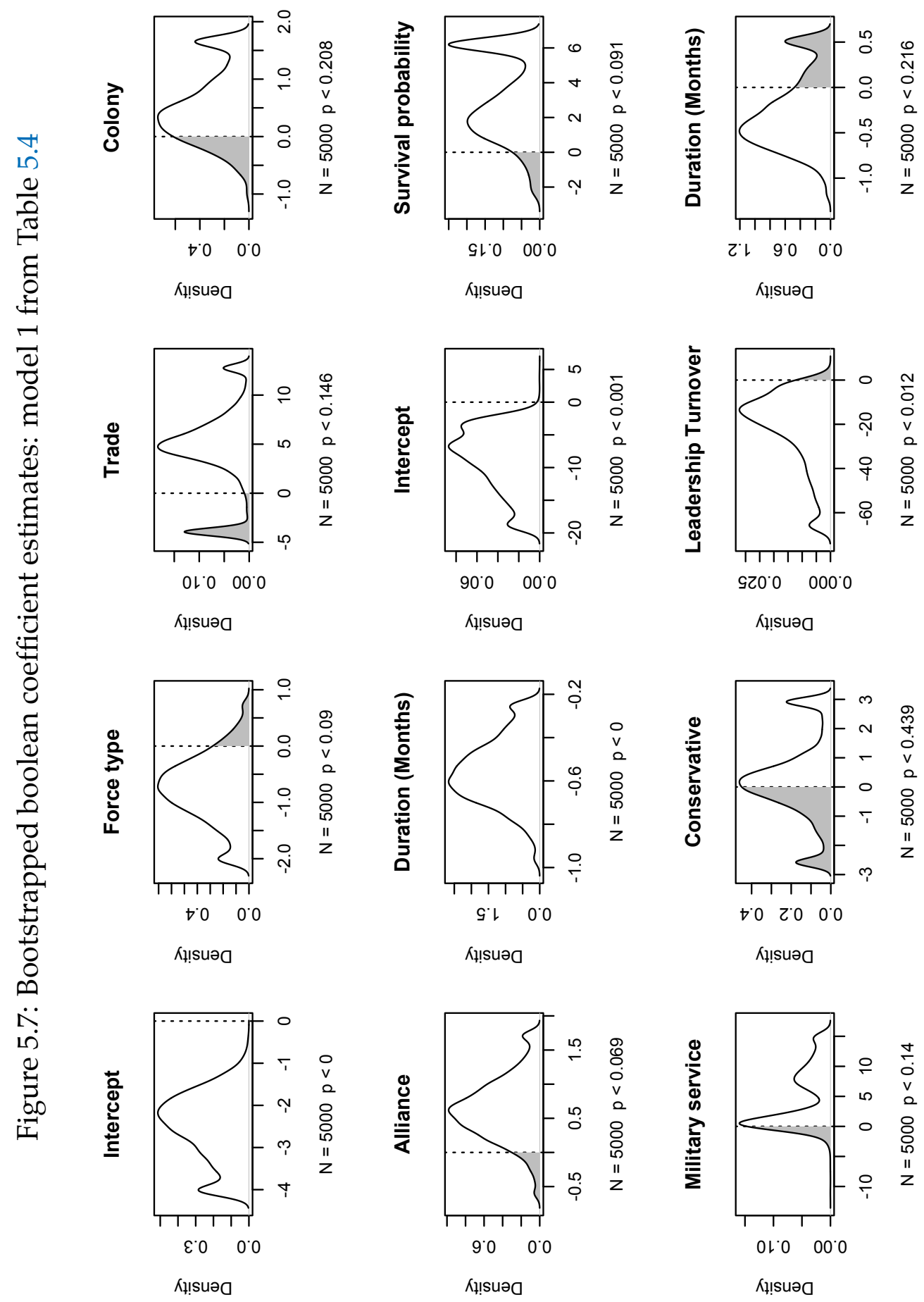

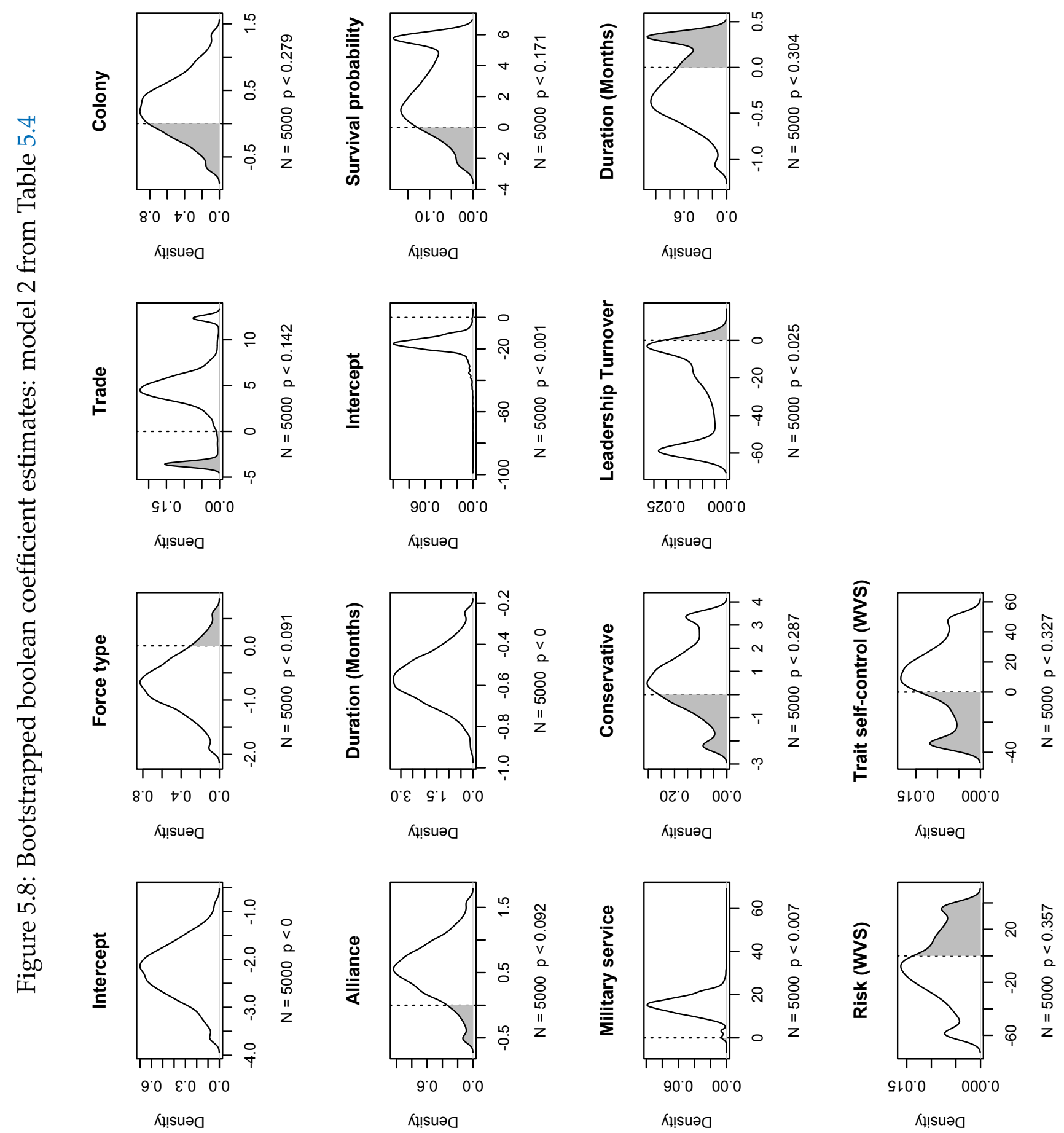
patterns in the results are familiar, although the baseline predicted probabilities are lower (as are the p-values), especially for the dispositional variables. Starting with the situational variables, the cost of fighting decreases the probability of victory, with an increase from the minimum to the maximum force type associated with a $1.0-1.1 \%$ decrease in the probability of victory $(p<0.09$ to $p<0.091$, depending on the model). Trade flows are associated with a substantively larger but statistically weaker impact: moving from the minimum to maximum levels of trade between the intervening power and the location of the intervention is associated with a $36.3 \%$ to $41.1 \%$ increase in the probability of victory, although failing to achieve conventional standards of statistical significance $(p<0.142$ to $p<0.146)$. As before, the current or former colonial status of the location of the intervention relative to the intervening power has no significant impact on the probability of victory, but alliance ties are associated with a $0.7 \%$ to $0.8 \%$ increase in the probability of victory ( $p<0.069$ to $p<0.092$ ). The dispositional effects are substantively weaker: an increase from the minimum to maximum leaders' probability of survival is associated with only a $0.22 \%$ to $0.66 \%$ increase in the probability of victory ( $p<0.091$ to $p<0.171)$, while military service is associated with a $0.02 \%$ to $0.04 \%$ increase ( $p<0.007$ to $p<0.14$ ). Conservatism, our measure of honor culture, is one again unrelated to the probability of victory - a fate similar to the societal-level risk and trait self-control variables - while an increase from the minimum to the maximum rate of leadership turnover is associated with a $4.5 \%$ to $5.3 \%$ decrease in the probability of victory $(p<0.012$ to $p<0.025)$.

The overall dynamics of the substitutable models are illustrated by the fitted value plot in Figure 5.9, which plots the fitted values from model 1 of Table 5.4. The plot shows that as our situational resolve measure increases, the probability of victory increases as well, while as the dispositional resolve measure increases, so too does the probability of victory. At the same time, though, the asymmetric tural counterparts, substitutable versions of models 2 and 4 from Table 5.2 failed to converge altogether. As before, the models are estimated with 5000 clustered bootstraps, each of which relies on a L-BFGS-B optimizer, with box constraints set to $\pm 3 \sigma$. 
Figure 5.9: Fitted values: substitutable causation

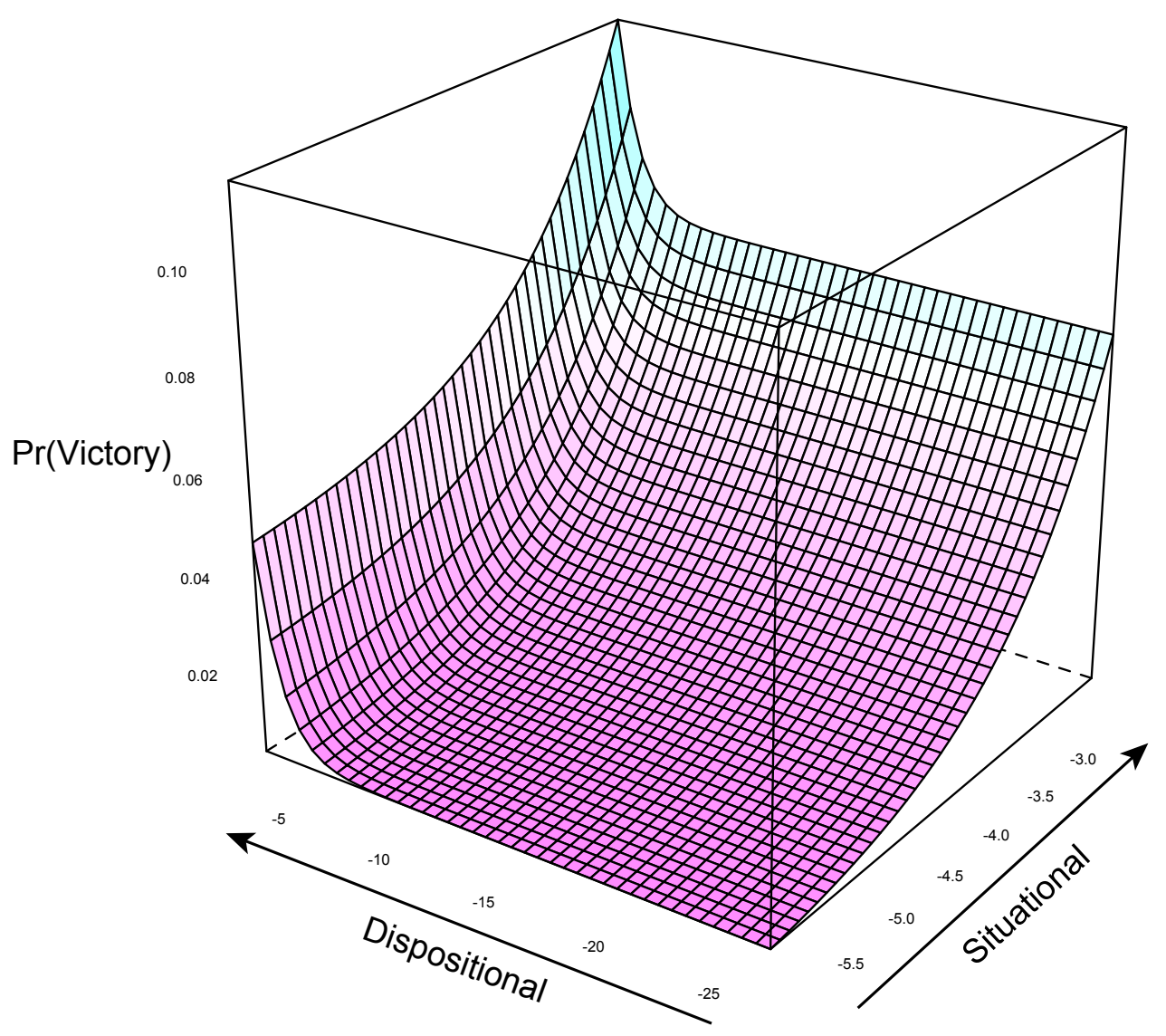

The fitted values of the dispositional and situational resolve scores from the substitutable model reveal a different dynamic than that of Figure 5.6. As before, as actors' dispositional resolve increases, so too does their likelihood of attaining their primary mission objective; the same can be said for increases in their situational resolve scores. However, since victory here is modeled as a function of situational or dispositional resolve, the overall shape of the plane differs from Figure 5.6: the probability of victory is relatively high if dispositional resolve is high, even if situational resolve is low (and vice versa). The fitted value scores here are derived from Model 1 in Table 5.4, clipped at the 2 nd and 98th percentiles to mitigate outliers. 
curvature of the plot surface shows that increases in the situational sources of resolve have substantively larger effects than those of the dispositional sources. AIC and BIC scores slightly favor the substitutable models in Table 5.4 over their conjunctural counterparts in Table 5.2, but the differences are relatively small once the two models include the same set of covariates, ${ }^{30}$ such that there is no decisive evidence in favor of one type of Boolean model against the other. The key question, though, is not how the conjunctural and substitutable models fare against one another, but how these two Boolean models compare with a traditional logit model, in which the effects of situational and dispositional resolve are additive rather than conjunctural or substitutable.

\section{How Well do the Boolean MOdels PREDict CONFLICT OUTCOMES?}

Table 5.5 re-estimates the four conjunctural models from model 5.2, but with traditional logistic regression rather than Boolean logit. The general pattern of results are similar - the probability of victory increases as a function of the countrylevel costs of backing down as measured by trade flows and alliance ties, and increases with leader-level time and risk preferences as measured by survival probabilities, leadership turnover, and military service. More important for our purposes, though, is that although the fit of the additive logit models are superior to the conjunctural Boolean logit models when measured by AIC and BIC scores, the additive logistic model has far lower predictive power than either of its Boolean counterparts. ${ }^{31}$ This point is illustrated by the Receiver Operating Characteristic (ROC) curves in Figure 5.10, which plots the true positive rate against the false positive rate for three models with identical covariates: a conjunctural Boolean logit model

\footnotetext{
${ }^{30}$ The AIC and BIC scores in Table 5.4 partly differ from those in Table 5.2 because they exclude the minimum distance variable; when an alternative version of the conjunctural model from Table 5.2 is estimated without minimum distance (as in the predictive model fit calculations below), it still has a slightly inferior fit to the substitutable model, with an AIC score of 744.55 (versus 741.96) and a BIC score of 817.81 (versus 815.22), although based on the criteria outlined in Raftery $(1995,139)$, BIC differences of 2 or less are considered weak.

${ }^{31}$ I use "prediction" here to refer to in-sample prediction rather than out-of-sample forecasting. For a review of out-of-sample forecasting in IR, see Schneider, Gleditsch, and Carey (2011).
} 
in which the probability of victory is based on situational and dispositional resolve, a substitutable Boolean logit model in which the probability of victory stems from situational or dispositional resolve, and a traditional logit model in which the odds of victory are simply an additive function of situational and dispositional resolve. ${ }^{32}$ The ROC plot shows that Boolean models far outpredict the traditional logit model, in that their true positive rates are consistently higher than their false positive rates, as reflected in their bowed shape. The regular logit model, on the other hand, has a false positive rate consistently higher than its true positive rate!

Figure 5.10: ROC curves for evaluating model predictive power

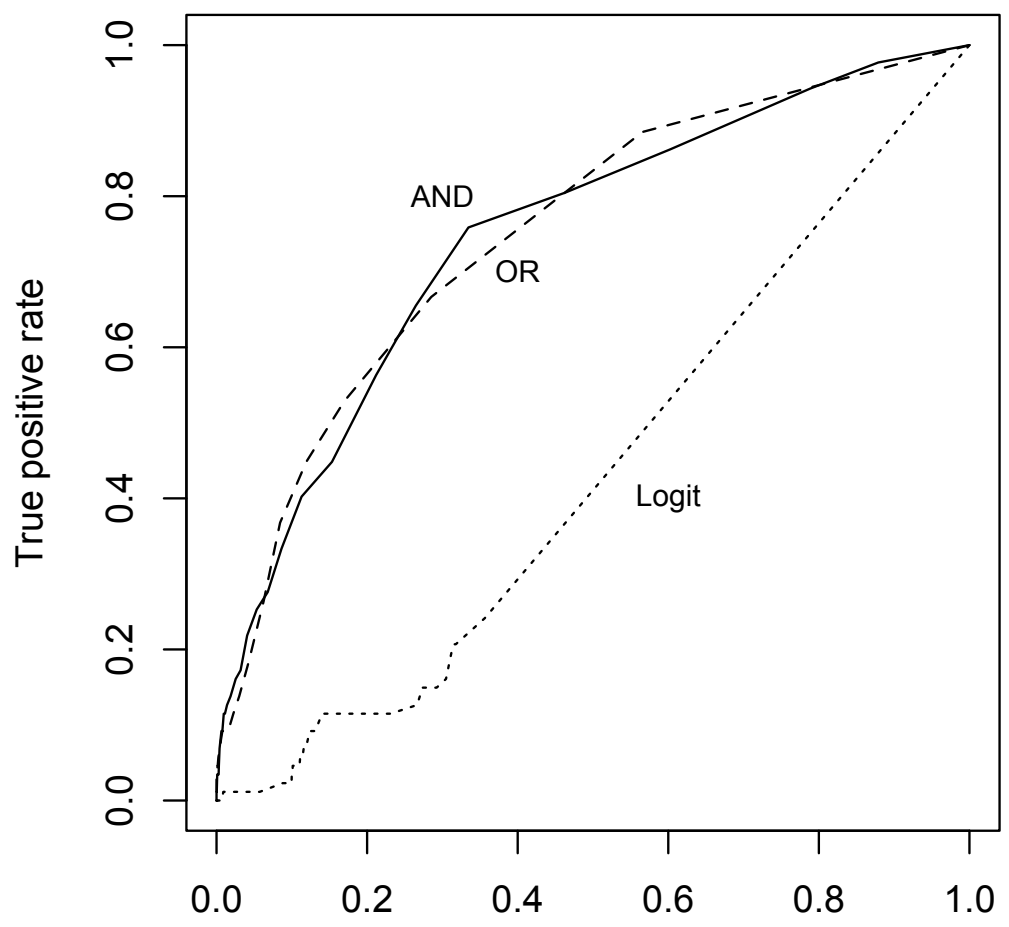

False positive rate

A comparison of ROC curves shows that the two Boolean models have far higher predictive power than the regular logit model: their true positive rates are consistently higher than their false positive rates, hence their bowed shape. The ROC curve for the regular logit, on the other hand, suggests that for many values of $\tau$, the logit model makes predictions worse than would be due to chance alone.

\footnotetext{
${ }^{32}$ The models are equivalent to model 1 from Table 5.2 without the minimum distance variable, model 1 from Table 5.3, and model 1 from Table 5.4.
} 
Table 5.5: Logistic model estimates: situation + disposition

\begin{tabular}{|c|c|c|c|c|c|c|c|c|}
\hline & \multicolumn{2}{|c|}{ (1) } & \multicolumn{2}{|c|}{ (2) } & \multicolumn{2}{|c|}{ (3) } & \multicolumn{2}{|c|}{ (4) } \\
\hline & $\beta *$ & $p *$ & $\beta *$ & $p *$ & $\beta *$ & $p *$ & $\beta *$ & $p *$ \\
\hline Intercept & $\begin{array}{l}-3.105 \\
(1.038)\end{array}$ & 0 & $\begin{array}{l}-3.123 \\
(1.147)\end{array}$ & 0 & $\begin{array}{l}-2.421 \\
(2.559)\end{array}$ & 0.164 & $\begin{array}{l}-2.608 \\
(2.667)\end{array}$ & 0.154 \\
\hline Force type & $\begin{array}{c}-0.46 \\
(0.413)\end{array}$ & 0.138 & $\begin{array}{c}-0.476 \\
(0.420)\end{array}$ & 0.118 & $\begin{array}{c}-0.465 \\
(0.451)\end{array}$ & 0.139 & $\begin{array}{c}-0.47 \\
(0.455)\end{array}$ & 0.143 \\
\hline Minimum Distance & $\begin{array}{c}-0.013 \\
(0.512)\end{array}$ & 0.498 & $\begin{array}{l}-0.015 \\
(0.517)\end{array}$ & 0.492 & $\begin{array}{c}0.32 \\
(0.642)\end{array}$ & 0.289 & $\begin{array}{c}0.290 \\
(0.657)\end{array}$ & 0.314 \\
\hline Trade & $\begin{array}{c}5.240 \\
(8.471)\end{array}$ & 0.066 & $\begin{array}{c}5.376 \\
(8.627)\end{array}$ & 0.07 & $\begin{array}{c}4.420 \\
(7.822)\end{array}$ & 0.102 & $\begin{array}{c}4.962 \\
(9.290)\end{array}$ & 0.107 \\
\hline Colony & $\begin{array}{c}0.133 \\
(0.313)\end{array}$ & 0.336 & $\begin{array}{c}0.149 \\
(0.328)\end{array}$ & 0.309 & $\begin{array}{c}0.376 \\
(0.384)\end{array}$ & 0.154 & $\begin{array}{c}0.35 \\
(0.403)\end{array}$ & 0.196 \\
\hline Alliance & $\begin{array}{c}0.542 \\
(0.295)\end{array}$ & 0.026 & $\begin{array}{c}0.527 \\
(0.299)\end{array}$ & 0.034 & $\begin{array}{c}0.482 \\
(0.335)\end{array}$ & 0.068 & $\begin{array}{c}0.458 \\
(0.341)\end{array}$ & 0.085 \\
\hline 曾 Leader Culpable & & & $\begin{array}{c}0.036 \\
(0.365)\end{array}$ & 0.458 & & & $\begin{array}{c}0.005 \\
(0.367)\end{array}$ & 0.502 \\
\hline 畨 Survival probability & $\begin{array}{c}1.046 \\
(0.940)\end{array}$ & 0.071 & $\begin{array}{c}1.048 \\
(0.992)\end{array}$ & 0.073 & $\begin{array}{c}1.000 \\
(0.881)\end{array}$ & 0.078 & $\begin{array}{c}1.017 \\
(0.882)\end{array}$ & 0.071 \\
\hline 胃 Military service & $\begin{array}{c}0.452 \\
(0.342)\end{array}$ & 0.090 & $\begin{array}{c}0.443 \\
(0.342)\end{array}$ & 0.08 & $\begin{array}{c}0.400 \\
(0.355)\end{array}$ & 0.126 & $\begin{array}{c}0.413 \\
(0.356)\end{array}$ & 0.118 \\
\hline 畨 Conservative & $\begin{array}{c}-0.082 \\
(0.334)\end{array}$ & 0.412 & $\begin{array}{l}-0.076 \\
(0.343)\end{array}$ & 0.419 & $\begin{array}{c}-0.012 \\
(0.349)\end{array}$ & 0.488 & $\begin{array}{c}0.004 \\
(0.348)\end{array}$ & 0.498 \\
\hline 县 Leadership Turnover & $\begin{array}{c}-0.834 \\
(0.669)\end{array}$ & 0.08 & $\begin{array}{l}-0.871 \\
(0.635)\end{array}$ & 0.08 & $\begin{array}{l}-1.235 \\
(0.717)\end{array}$ & 0.039 & $\begin{array}{l}-1.202 \\
(0.747)\end{array}$ & 0.054 \\
\hline Risk (WVS) & & & & & $\begin{array}{c}-5.029 \\
(5.600)\end{array}$ & 0.180 & $\begin{array}{c}-4.632 \\
(5.698)\end{array}$ & 0.2 \\
\hline Trait self-control (WVS) & & & & & $\begin{array}{c}4.802 \\
(4.632)\end{array}$ & 0.142 & $\begin{array}{c}4.724 \\
(4.699)\end{array}$ & 0.158 \\
\hline Duration (Months) & $\begin{array}{c}-0.498 \\
(0.079)\end{array}$ & 0.000 & $\begin{array}{c}-0.495 \\
(0.101)\end{array}$ & 0.000 & $\begin{array}{c}-0.482 \\
(0.084)\end{array}$ & 0.000 & $\begin{array}{c}-0.481 \\
(0.101)\end{array}$ & 0.000 \\
\hline Conflict & 33 & & 33 & & 33 & & 33 & \\
\hline Interventions & 12 & & 12 & & 12 & & 12 & \\
\hline $\mathrm{AIC}$ & 742 & & 744 & & 745 & & 747 & \\
\hline BIC & 809 & & 817 & & 824 & & 832 & \\
\hline
\end{tabular}

denote leader-level variables. Main entries are coefficient estimates; standard errors in parentheses, estimated using 2000 cluster bootstraps. Bootstrapped p-values are one-sided to facilitate a direct comparison with the results from the Boolean models. 
The same findings are evident when we employ other predictive measures of model fit. The expected percent correctly predicted (ePCP) (Herron, 1999) for the Boolean AND model and OR models are $93.0 \%$ and $95.6 \%$, respectively (indicating that our Boolean models correctly assign $93.0 \%$ and $95.6 \%$ of their PDFs to the correct level of the dependent variable), whereas the $\mathrm{PCP}$ for the regular logit is only 87.1\%. Similarly, the Brier scores for the two Boolean models are 0.025 , whereas the Brier score for the regular logit model is 0.088. Separation plots (Greenhill, Ward, and Sacks, 2011) shown in Figure 5.11 show that the regular logit model consistently misclassifies observations, predicting failures to achieve objectives where victories in fact occur; the Boolean models misclassify observations as well, but at a lower rate - for both the AND and the OR models, the darker lines are concentrated towards the right-side of the separation plots. As was the case in the other measures of predictive fit, the two Boolean models perform about the same: the AND model seems to have more false negatives in the lower fitted values (the left-hand third of the plot), while the OR model seems to have more false negatives at moderate fitted values (the middle third of the plot). Thus, both the conjunctive and substitutable models perform equally well with respect to predictive fit, and far superior to the additive logit.

\subsection{Conclusion}

One of the chief impediments to studying resolve with observational data has been a lack of understanding about what resolve's microfoundations are. This neglect of microfoundations has been one reason why our treatment of resolve in International Relations has been dissatisfying: not only are our theories of resolve underspecified, but our empirical tests are frequently misspecified, because we do not know how to measure resolve with observational data, forcing us to either to drop resolve from our empirical models, or infer it from one of its posited consequences. Indeed, it is striking how much of the literature that invokes resolve either laments how hard it is to measure - Organski $(1968,208)$, like Sartori $(2005,91)$, claims it is 
Figure 5.11: Separation plots show the predictive superiority of the Boolean models

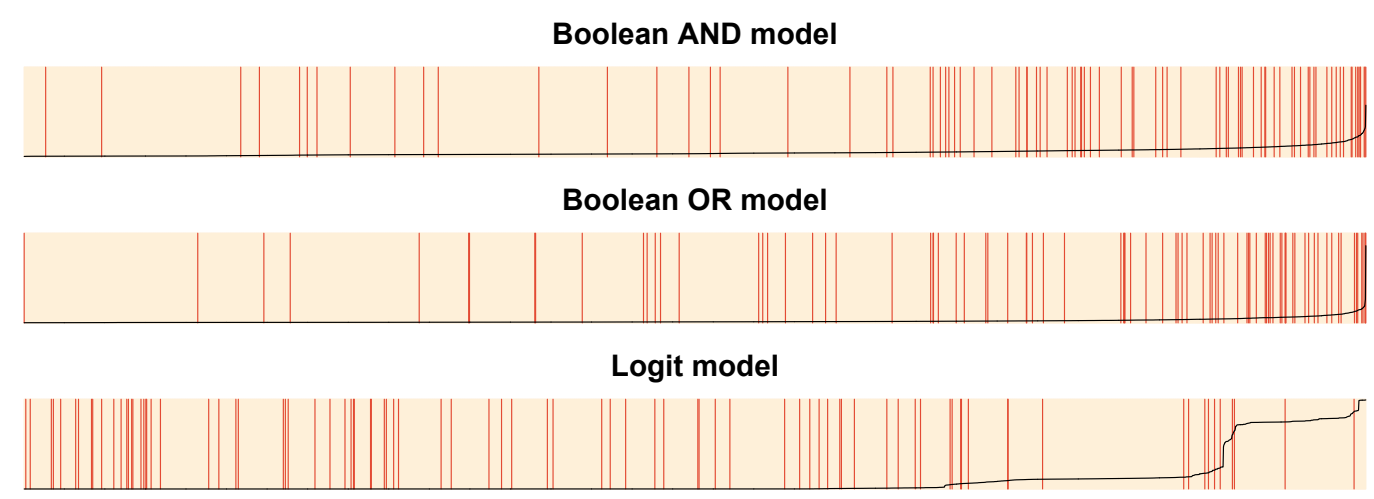

Separation plots (Greenhill, Ward, and Sacks, 2011) show that the conjunctive and substitutable Boolean models have higher degrees of predictive fit than the regular logit model. In a perfectly predictive model, the light and dark vertical lines representing the the $0 \mathrm{~s}$ and $1 \mathrm{~s}$ in the model would be perfectly separated from one another, with the light lines (representing observations where no victory occurs) clustered on the left, and the darker lines (representing observations where victory does in fact occur) clustered on the right. The black line shows the actual predicted values of each model.

"extremely difficult to measure objectively", Rummel $(1975 b, 318)$ calls it "the most elusive and ambiguous of psychological variables", and Cline $(1994,98)$ notes that the study of resolve is "a region where numbers can only be notations of highly subjective judgments, nothing more" - or complains that too many of the measures are derived ex post (March, 1966, 61; Baldwin, 1979; Jervis, 1979, 316; Ray and Vural, 1986; Morrow, 1989; Merom, 2003; Sullivan, 2007). Rather than study resolve based on its consequences, I do so based on its causes, gathering measures of the same constructs tested in the lab and survey experiments, and using them to build composite dispositional and situational measures of resolve in the context of great power military interventions from 1946-2003.

I therefore turn to Boolean statistics as a new way to answer an old question. The analyses suggest a number of findings. First, and most importantly, resolve and victory are indeed positively related: the situational and dispositional predictors of resolve also predict the probability that the great powers achieved their objectives in their military interventions from 1946-2003. This effect is important for three reasons. First, and most obviously, because the measures of resolve are de- 
rived from resolve's causes rather than its consequences, I am able to test resolve's effects without lapsing into tautology. Second, I am focusing solely on great power military interventions, which raises some interesting case selection implications. If great powers tend to have more resolve than other states - either because their resolve is what prompts them to become great powers in the first place, or because they tend to select more resolute leaders because of the frequency with which great powers tend to be involved in international crises - I am likely to be studying the impact of resolve in relatively high-resolve cases, thereby rendering my tests of resolve's effects relatively conservative. The fact that I still find that resolve makes a difference despite this ceiling effect suggests that resolve's impacts on conflict outcomes are likely to be even larger than what I find here. Third, and relatedly, the situational determinants of resolve I study here are observable ex ante - when deciding whether to intervene in the first place, decision-makers are aware of how far away the intervention would be taking place, whether the intervention would be taking place in an ally, and so on - and thus likely to factor into the initial decision of whether to intervene in the first place. Since I am not explicitly modeling this selection process, this ex ante observability raises the possibility that my estimates of the overall impact of resolve are biased. As I note earlier in the chapter, however, the downward direction of this bias would make the impact of resolve harder to detect, thereby rendering my tests more conservative, and the significant relationship between resolve and conflict outcomes all the more noteworthy.

Second, of the situational variables, I find fairly consistent evidence for the importance of the costs of backing down - both the intrinsic interests at stake, and reputation costs - but less support for the importance of the costs of fighting. This null finding could be due either to better measures of the former than the latter, or to selection effects, in that the cost of fighting may be taken into account when deciding whether to intervene in the first place, and thus have little impact on the probability of actually achieving military outcomes - similar to the effect we see in the mediation analyses of anticipated costs in the survey experiment. The impor- 
tance of reputation costs shows that even if decision-makers worry more about their national reputation than they should (Mercer, 1996; Press, 2005), these concerns do seem to guide their behavior: when the reputational consequences of walking away short of victory are higher, actors are more likely to emerge victorious.

Third, with respect to the dispositional variables, we see fairly strong evidence that the probability of victory increases with leaders' time preferences, and moderately supportive evidence that it increases with leaders' risk preferences, but no evidence in favor of honor culture, societal-level risk-attitudes, or trait self-control. The novel contribution of these analyses comes not from the individual variables themselves - none of which are particularly exotic in the study of peace science but from the overarching situational and dispositional framework in which they are incorporated. Thus, I find different patterns of effects for the situational and dispositional sources of resolve: when it comes to situational variables, the most relevant level of analysis for resolve seems to be that of the country as a whole, but for dispositional variables, the most relevant level of analysis appears to be the leader-level.

Finally, I modeled resolve here using two different types of Boolean models a conjunctural model in which military outcomes are function of both situational and dispositional resolve, and a substitutable model in which outcomes are a function of either situational or dispositional resolve; analyses show that the two sets of Boolean models have far higher predictive power than a traditional logit model, reconfirming the importance of acknowledging causal complexity (Braumoeller, 2003) in our models of conflict. Both models, then, suggest that scholars looking at resolve in the context of military interventions should pay attention to both situational and dispositional factors - in the conjunctural model because dispositional factors moderate the impact of the cost of war, and in the substitutable model because dispositional resolve can boost the odds of victory even when situational resolve is low. ${ }^{33}$

\footnotetext{
${ }^{33}$ Given similar predictive fits, which type of model should one choose to estimate? Two such criteria to consider are theoretical expectations, and the stability of the coefficient estimates. In this
} 
Several points should be noted here. First, as discussed above, these findings about levels of analysis could simply be due to better leader-level dispositional measures than country-level ones in that the former are time-varying wheres the latter are not. However, in emphasizing that our dispositional characteristics of interest are variables rather than constants, this pattern of findings nonetheless has implications for how we think about dispositions in IR: not as fixed and enduring national characteristics similar to early discussions in IR about "national character" (Charlesworth, 1967; Hoebel, 1967) or "national style" (Gray, 1981), but as evolving entities. Thus, although country-level fixed effects might capture unit heterogeneity, they would do a poor job of capturing dispositional characteristics. Meanwhile, the importance of leader-level characteristics in the findings speaks to a growing body of research showing that the importance of studying phenomena at the leader-level rather than simply the country-level as a whole (Hermann et al., 2001; Chiozza and Goemans, 2011; Jervis, 2013). The evolving nature of leader-level dispositional variables also suggests that leader dispositions are better modeled theoretically rather than controlled for using leader-level fixed effects.

Second, many of the substantive effects reported here may seem small - for example, previous military experience's substantively largest effect in Table 5.2 is only a $2.05 \%$ increase in the probability of victory. However, these small changes in predicted probability reflect the relatively low baseline probability of victory at any given point in time - as is common in quantitative studies of war (e.g. King and Zeng 2001). Indeed, if we express these substantive effects in terms of odds ratios rather than predicted probabilities, their substantive magnitude appears far greater: leaders with previous military experience (and thus, greater risk acceptance) are 2.4 times likelier to prevail than those without.

Third, it is important to acknowledge that many of the situational and dispositional measures I employ are relatively rough proxies, such that they may operate through alternative mechanisms. For example, previous military experience might case, both such criteria favor a conjunctive model over a substitutable one. 
increase the probability of victory not through greater risk acceptance, but because more experienced leaders may be better at selecting interventions they know they can win. This point is important to acknowledge, and in the following chapter, I suggest how the use of text as data may provide a more direct way of estimating leader and country-level dispositional characteristics in future research. 


\section{Chapter 6: Conclusion: Solving for $x$}

In War and Peace, Tolstoy analyzes the mechanics of war by using an analogy to physics, noting that "in warfare the force of armies is the product of the mass multiplied by something else, an unknown $x .^{\prime 1}$ The unknown $x$, he later explains, is resolve, a variable subjected to no less scrutiny by twenty-first century political scientists and policymakers than nineteenth century Russian novelists. Given the extent to which political science scholarship in general - and IR scholarship in particular — is often divided by both methodological and substantive sects (e.g. Lake, 2011), resolve's predominance presents something of a paradox. On the one hand, it is a central protagonist in all quadrants of the field, looming large in everything from classical qualitative accounts of 'national will' as a component of power (Wright, 1964; Organski, 1968; Morgenthau, 1985), to seminal game theoretic models of crisis bargaining and battlefield outcomes (Snyder and Diesing, 1977; Fearon, 1995; Smith and Stam, 2004; Powell, 2004), to influential quantitative studies examining whether democratic publics are really as irresolute or cost-sensitive as policymakers have often feared (Mueller, 1971; Bennett and Stam, 1998; Schultz, 2001b; Reiter and Stam, 2002; Gelpi, Feaver, and Reifler, 2009). On the other hand, I argued here that despite its ubiquity in the discipline, resolve very much remains an "unknown $x^{\prime \prime}$ : conceptually, we do not know what it is, and tend to use it in a variety of inconsistent ways, often conflating it with capabilities and intentions. Empirically, we do not know how to measure it, and tend to either relegate it to the error term, or infer it tautologically from the outcomes we're using it to explain. Theoretically, we do not know where resolve comes from, and why some actors are more resolved than others.

\footnotetext{
${ }^{1}$ Part XIV, Chapter II, cited in Morgenthau $(1985,151)$
} 
Following Waltz $(1959,11)$, who noted that there is no "self-denying ordinance against the use of materials and techniques of other social scientists," I sought to address these questions by connecting work on resolve in IR with a growing body of research on willpower and self-control from elsewhere in the social sciences, arguing that "political will" is not just a figure of speech: the same dispositional characteristics that social psychologists and behavioral economists use to predict willpower in our daily lives also moderate the impacts of the costs of war, and thus can be used to explain resolve in military interventions. I tested the theory with multiple methods, at multiple levels of analysis: first, using lab and survey experiments to study the determinants of resolve at the individual-level in the context of public opinion about military interventions, and subsequently using the experimental results as individual-level microfoundations for a series of country- and leader-level Boolean analyses of resolve in the context of great power military interventions.

Table 6.1: Comparison of results: experimental and observational data

\begin{tabular}{|c|c|c|c|}
\hline & \multicolumn{3}{|c|}{ Dependent variable } \\
\hline & \multicolumn{2}{|c|}{ Resolve } & \multirow{2}{*}{$\begin{array}{l}\text { Military outcomes } \\
\text { Boolean analyses }\end{array}$} \\
\hline & $\mathrm{Lab}$ & Survey & \\
\hline \multicolumn{4}{|l|}{ Situational variables } \\
\hline \multicolumn{4}{|l|}{ Cost of fighting } \\
\hline Ex ante & $\checkmark$ & $x$ & * \\
\hline Ex post & $\checkmark$ & $x$ & \\
\hline \multicolumn{4}{|l|}{ Cost of backing down } \\
\hline Intrinsic (stakes) & & & $\checkmark$ \\
\hline Extrinsic (reputation) & * & $\checkmark$ & $\checkmark$ \\
\hline \multicolumn{4}{|l|}{ Dispositional variables } \\
\hline Time preferences & $\checkmark$ & $\checkmark$ & $\checkmark$ \\
\hline Risk preferences & $\checkmark$ & $\checkmark$ & $\checkmark$ \\
\hline Honor culture & $\checkmark$ & $x$ & $x$ \\
\hline Trait / behavioral self-control & $\checkmark$ & $\checkmark$ & $x$ \\
\hline Interactionist dynamics & $\checkmark$ & * & $\checkmark$ \\
\hline
\end{tabular}


On the whole, the results, summarized in Table 6.1 offer relatively consistent evidence in favor of the importance of the costs of backing down, but less so with the costs of fighting. As evident in Table 6.1, the evidence for the impact of the cost of fighting is mixed: both anticipated costs and casualties significantly impact resolve in the lab experiment, but not the survey experiment, and the impact of force type on the probability of victory in the large- $\mathrm{N}$ analyses is relatively weak. The evidence for the impact of the cost of backing down is greater, for both reputation costs (tested in both the experiments and the large-N analyses) and the interests at stake (tested solely in the large-N analyses). Thus, although there is relatively weak evidence supporting the hypothesis that the costs of fighting lowers the amount of resolve displayed (and thus, decreases the probability of victory), there is stronger evidence in favor of the contention that the costs of backing down increases the amount of resolve displayed (and thus, increases the probability of victory).

This pattern of results has implications for three different literatures. First, in showing that resolve should not simply be treated as the inverse of an actor's costs of fighting, the findings challenge one of the working assumptions of the rationalist IR literature, which has tended to use the "costs of war" as a shorthand for resolve; across the empirical chapters, the cost of fighting appears to be neither the only nor even the predominant source of resolve. Second, the results also speak to the public opinion literature on the use of force, especially the "cost-benefit" model of public opinion (Larson, 2000; Klarevas, 2002), in that the benefits appear to offer higher explanatory power than the costs (Gelpi, Feaver, and Reifler, 2009). In this respect, although the public opinion literature has tended to focus quite heavily on casualties as a major determinant of public opinion about the use of force (e.g. Mueller, 1971; Gartner and Segura, 1998; Gartner, 2008a), they play less of a role here. One potential explanation for this discrepancy is that much of the experimental work that studies casualties tends to focus upon it in isolation, manipulating either relative casualties (Boettcher and Cobb, 2006), absolute casualty levels and trends (Gartner, 2008a), or secondary casualty information (like the number of 
female casualties) (Gartner, 2008b), collectively producing rich and detailed portraits of the different ways in which casualties affect attitudes towards conflict, but giving relatively little sense of the substantive importance of casualties relative to other factors. Meanwhile, although analyses of attitudes towards the use of force using observational data have tended to avoid this pitfall, the fact that casualties always accumulate as interventions proceed (Smith, 2005) mean that casualties are one of many factors correlated with time, making its relative effect difficult to disentangle (Althaus and Coe, 2011, 74; Althaus, Forthcoming). Third, these dynamics also speak to a well-established literature evaluating the public's prudence (Jentleson, 1992), rationality (Page and Shapiro, 1992), and consistency (Almond, 1950; Converse, 1964). One of the striking points about the lab and survey experiments is that they consistently fail to find evidence in favor of "sunk cost" logic driving participants' attitudes towards military interventions. Since sunk cost effects are, as Arkes and Blumer $(1985,124)$ note, "irrational," these results paint a relatively positive picture of the public.

The results for the dispositional variablesshow that war is not just something that takes place on the battlefield, but also takes place "in between the ears." In particular, we consistently see strong effects for time and risk preferences. Longer time preferences are associated with greater resolve in both the lab and survey experiments, and longer leader time horizons bolster the probability of victory in the large-N analyses. Although the functional form of risk attitudes changes - a significant nonlinear association with resolve in the experimental data, and a significant linear relationship with the probability of victory at the leader-level in the observational data - it consistently exerts a significant impact. ${ }^{2}$ Relatively weak evidence is found for honor culture, which only has a significant effect in the lab experiment, and neither affects resolve in the survey experiment, nor the probability of victory in the large-N analyses. Trait or behavioral self-control has a slightly better fate,

\footnotetext{
${ }^{2}$ As noted in the previous chapter, the fine-grained continuous measure of risk attitudes in the experimental data is conducive to detecting nonlinear relationships, whereas the dichotomous measures used in the observational data preclude such a possibility.
} 
producing significant effects in the experimental data, but not the observational data. Given that the experiments employ better measures of trait self-control than the large- $\mathrm{N}$ analyses, it is possible that trait self-control indeed bolsters the probability of victory, but we are unable to find support for this hypothesis with the existing data. Since dispositional variables implicate an interesting level-of-analysis question, it is interesting to note that in the large- $\mathrm{N}$ analyses, the dynamic leaderlevel dispositional measures consistently perform better than static country-level ones, suggesting we should think of dispositions in IR as evolving rather than fixed entities, and focus more on individual leaders as the relevant unit of analysis rather than countries as a whole. These results should thereby lend some skepticism about IR theories that rely on national cultural generalizations (e.g. Huntington, 1993) rather than invoking more contingent or fine-grained accounts of dispositional tendencies (e.g. Hopf, 2002).

At a broader level, I also find evidence in favor of an interactionist theory of resolve - tested in the lab and survey experiments using a series of interactions between situational treatments and dispositional features, and in the large- $\mathrm{N}$ analyses by a Boolean statistical model, in which the probability of victory is modeled as the product of situational and dispositional features - thus taking the exact functional form that Tolstoy predicts when he envisions material (situational) features being multiplied by $x$ in War and Peace. Importantly, this Boolean model displays a far higher predictive fit than a standard linear additive model, which is also outperformed by a substitutable Boolean model in which resolve is modeled as a function of either situational or dispositional features. Indeed, as noted in Chapter 5, the novel contribution of the project comes not from the individual variables in the analyses, but the ends to which they are applied and the overarching framework in which they are incorporated.

More important, though, is the following. Political scientists have long lamented our failure to subject theories of resolve to serious empirical scrutiny. As Ray and Vural $(1986,321)$ complained, "If there were a dependable, widely accepted way 
to measure the "will to win," silly arguments and their advocates could not escape detection so easily, and perhaps not at all." The tests in Chapters 3-5 suggest that the argument that resolve boosts the likelihood of victory is not so silly after all. That said, the dispositional side of resolve to which Ray and Vural are referring here is not as all-powerful as some of its boosters have claimed. Napoleon's famous dictum that in war, moral factors outweigh material factors by a ratio of three to one, for example, is not borne out by the data. If we think of dispositional sources of resolve as moral factors, and situational sources of resolve as material factors, the slopes of the $\mathrm{x}$ and $\mathrm{z}$ axes in Figures 5.6 and 5.9 show that both factors are important, but the slope of the fitted values along the dispositional axes are not three times higher than along the situational ones.

By highlighting the importance of causal complexity in thinking about social phenomena (Braumoeller, 2003) and avoiding what Morgenthau $(1985,174)$ called "the fallacy of the single factor," the results will likely disappoint scholars accustomed to understanding social phenomena as the function of a single treatment and a host of substantively uninteresting control variables. The findings will also be troubling for policymakers looking for a monocausal explanation for resolve. Indeed, the results suggest there is no "magic bullet" for resolve: no single variable that political scientists and policy-makers need to pay attention to in order to predict when some actors will be display more resolve than others.

Rather than rehashing the main points outlined in the previous pages, I turn instead to a number of extensions, qualifications, and areas for future research. First, I speculate about the theory's applicability to other domains. I then discuss how the findings raise questions about two common assumptions in IR theory - the extent to which resolve is "private information" that decision-makers can access but foreign rivals cannot, and the dichotomy between capabilities and resolve in classical theories of war. I then suggest a number of ways resolve could be studied in a dyadic rather than a monadic fashion, and raise a normative question regarding the ethics of resolve. I subsequently revisit the situational versus dispositional dis- 
tinction, noting how the use of text as data can offer a more direct way of measuring leaders' dispositional features. Finally, I conclude by responding to a potential objection from strong dispositionalists, and suggesting a number of additional causes of resolve that could be incorporated into future studies.

\subsection{Resolve in other domains}

Although the theory of resolve advanced here is deliberately general, all of the empirical tests focus explicitly on the context of resolve in military interventions. In this sense, although the results from Chapters 3-5 should not be used to evaluate claims about the dynamics of resolve in other contexts (for example, Krauthammer's (2009) assertion that sufficient resolve can reverse the decline of American unipolarity), the overarching framework itself easily applies and lends itself to future tests. For example, in the study of International Political Economy (IPE), "political will" is often used to explain phenomena like participation in IMF programs (Przeworski and Vreeland, 2000) and the implementation of cooperative international agreements like TRIPS (Levy, 1999). Although the costs of participation and the costs of backing down are different in an IPE context than an international security one, the basic framework still holds, as does the importance of dispositional variables like time and risk preferences.

\subsection{How private is private information?}

One key implication of the above findings is that if resolve is indeed a function of both situational stakes and dispositional traits, it may be worth revisiting our standard understanding of resolve in rationalist IR as private information that (i) decision-makers can access but (ii) foreign rivals cannot (e.g. Goemans 2000). My results challenge these assumptions on two contradictory fronts.

On the one hand, if resolve is indeed a state rather than a trait, an argument might be made that it is unclear that decision-makers should be able to know their 
own country's resolve ex ante, since it is a function of both situational and dispositional causes existing at multiple levels of analysis. ${ }^{3}$ In this understanding, conflict and crisis bargaining are not merely about finding out your opponent's level of resolve (Van Evera, 1999, 27-8), but about discovering your own resolve as well. This finding comports with research on willpower in social psychology and economics; in their study of willpower and personal rules, Bénabou and Tirole $(2004,159)$ base their model on the assumption is that "people have limited knowledge of their strength or weakness of will", consistent with a bevy of findings about self-serving and inaccurate assessments of one's own dispositions (e.g. Klein and Kunda, 1993; Epley and Dunning, 2000; Dunning, Heath, and Suls, 2004), and the tenets of selfperception theory, which argues that we are forced to make inferences about our own traits by observing our behavior (Bem, 1967). If this is the case, war does not just involve learning about your opponent's reservation point, but learning about your own as well. ${ }^{4}$

On the other hand, even if resolve itself is not directly observable, the premise for the large- $\mathrm{N}$ analyses in Chapter 5 is that resolve has observable causes. Certainly, many of the proxies employed in the previous chapter - rates of leadership turnover, leaders' previous military experience, alliance commitments, and so on — are observable not just by analysts, but by decision-makers themselves, not just ex post, but ex ante. Yet if information about these factors are available ex ante, then this private information is in fact public information, and uncertainty about resolve can no longer explain why rational actors would fight in the first place. One potential explanation is that not all of these indicators are always public: alliances through the nineteenth and early twentieth centuries were often secret, for example - as reflected in the first of Woodrow Wilson's Fourteen Points, which called for "open covenants openly arrived at." Yet this caveat does not apply to the 126 interventions investigated in the previous chapter, and from a rationalist perspective,

\footnotetext{
${ }^{3}$ This possibility is also raised by Morgan $(1990,280)$, as well as Walt $(1999,34$, fn 85$)$ in his critique of audience cost models.

${ }^{4}$ To be sure, psychologists argue we know more about ourselves than we do about others (Chambers et al., 2008), but this is a relatively low baseline.
} 
the fact that these interventions took place suggests that the interventions' targets and initiators failed to accurately gauge each others' resolve. A more plausible explanation is that these measures are relatively rough, and do not perfectly predict resolve, such that there is still substantial room for error. Another is that although this information might be observable ex ante, leaders might not avail themselves of it; indeed, as Morgan $(1990,280)$ points out, this is a broader concern for game theoretic approaches, in that "if the players know the situation and the theory, they can predict with certainty the outcome and should not waste their time "bargaining."' I may calculate fitted values from Boolean logistic regressions on situational and dispositional indicators of actors' resolve, but it is likely unrealistic to presume decision-makers do the same, rather than engaging in satisficing (Simon, 1957) or motivated reasoning (Kunda, 1990).

\subsection{Resolve and capabilities}

These findings also question some of the classical understandings about the relationship between capabilities and resolve. In the interactionist framework advanced here, capabilities are a source of resolve, in as much as increases in capabilities reduce the cost of fighting. If this framework is correct, it makes little sense to treat capabilities and resolve as competing explanations for military outcomes (e.g. Maoz, 1983; Ray and Vural, 1986); accounts that attempt to do so are really contrasting the explanatory power of a particular situational source of resolve versus a dispositional source, such that the real dichotomy is not between capabilities and resolve, per se, but between situational and dispositional features.

Similarly, the classical realists who argued that "national morale" was a source of power (e.g. Wright, 1964; Morgenthau, 1985) have the causal arrow reversed: rather than resolve bolstering power, power increases resolve. Claims about national morale or national will as a source of power show that the power literature suffers from many of the same tautologies that the resolve literature does: if power is defined via the outcomes it is used to explain, as in Dahl's (1957) seminal defini- 
tion of power as " $A$ has power over $B$ to the extent that he can get $B$ to do something that $B$ would not otherwise do", it becomes impossible to empirically test the relationship between power and outcomes (since by definition, $A$ must prevail over $B$ if the former is more powerful than the latter). The "paradox of unrealized power" (Baldwin, 1979, 163) is only a paradox if we take this tautology at face value. ${ }^{5}$

In a sense, the move to studying power via material capabilities - exemplified by the Correlates of War project's focus on military expenditures and personnel, energy consumption, iron and steel production, and population (Singer, Bremer, and Stuckey, 1972) - represents a similar move to the one I employ here with respect to resolve in Chapter 5: power is a latent variable, so rather than study it based on its consequences, we do so based on its causes. Indeed, many of the most important variables in International Relations are also the hardest the measure: legitimacy, leadership, status, prestige, and so on. Like resolve, many of these variables tend to be studied based on their posited consequences in a manner that flirts with tautology. The two-stage measurement strategy I adopt here to study of resolve can be appropriated to study other such constructs as well.

An entrepreneurial reader might wonder whether the type of two-stage framework I employ here could be used to generate situational and dispositional resolve scores similar to the CINC scores in the Correlates of War, which could then be exported to a vehicle like EUGene (Bennett and Stam, 2000) for easy use in other studies. Although this would be appropriate for dispositional resolve scores, this is less practical for situational resolve scores, since situational resolve is relational to the costs inherent in a particular intervention, rather than monadic: for example, Chinese situational resolve during the Korean War was different than its situational resolve during its intervention in Burma on behalf of Chinese nationalists, even though the two military operations took place at the same time.

\footnotetext{
${ }^{5}$ Note that this is different from the distinction between "latent capabilities" and actual capabilities (e.g. Walt, 1985), which refers instead to countries that have large scale industrial production but have not converted it into military assets.
} 


\subsection{On the dyadic nature of resolve}

The relational nature of the situational resolve measures raises the question of the dyadic element to resolve. As noted in Chapter 5, we often understand resolve dyadically, in terms of a "balance of resolve", but for reasons of tractability, neither the analyses with experimental data in Chapters 3-4 nor the analyses with observational data in Chapter 5 have a dyadic dynamic. In the lab and survey experiments, rather than explicitly including information about the opponent's resolve in the experimental vignettes, players must infer the resolve of the opponent through events on the battlefield (Ramsay, 2008). If we sought to more explicitly study how these situational and dispositional predictors of resolve change in a dyadic experimental context, there are three ways we might consider doing so, although two of them involve substantial tradeoffs, and the third ends up being similar to the experimental designs employed in Chapters 3-4.

First, we could change the format of the experiment, from a vignette-based public opinion about foreign policy experiment in the tradition of Herrmann, Tetlock, and Visser (1999) to an economics-style bargaining game experiment where two players face off against one another (e.g. Dickson, 2009; Tingley and Walter, 2011). We can manipulate situational determinants of resolve via the payoff structure, and as before, measure participants' dispositional traits using standard instruments from behavioral economics and social psychology. In abstracting to a bargaining game, though, we lose the IR-specific content of the vignettes, the ability to make inferences about public opinion (since participants play as agents rather than principals (Laffont and Martimort, 2002)), and the context of a military intervention more specifically: the players are simply challengers and defenders rather than an intervening power seeking to repel the invasion of its smaller ally by its larger neighbor. Second, we could attempt to preserve the IR-specific content of the scenario through a crisis simulation game (e.g. McDermott and Cowden, 2001; Johnson et al., 2006), where participants are leaders deciding whether to attack, and how long to persist. Given what we know about the extent to which non-elite 
participants differ from actual decision-makers (Mintz, Redd, and Vedlitz, 2006; Kertzer and McGraw, 2010; Hafner-Burton et al., 2013), however, there is a tradeoff here as well. ${ }^{6}$ Alternately, borrowing from attribution theory - in which the resolve of another actor simply becomes part of your own situation - we could retain the original vignette-based public opinion-style experiment setup, but also manipulate the opponent's resolve. However, given that resolve is not directly observable, these manipulations of resolve essentially turn into manipulating events on the battlefield, thus producing something strikingly similar to the experimental designs employed in Chapters 3 and 4. More importantly, if the resolve of your opponent affects your resolve simply by affecting your costs of fighting, the more interesting question is not how your resolve is shaped by your opponent's resolve, but rather, how this balance of resolve affects actual conflict outcomes.

To extend the large- $\mathrm{N}$ analyses to capture this dyadic component, we need to consider two different challenges, but neither of which are insurmountable. First, we need to calculate dispositional and situational resolve scores for the target of the intervention. This requires overcoming data availability issues, especially for the dispositional sources of resolve, as well as some conceptual considerations involving the relevant situational variables for the target. Second involves deciding the best way to operationalize the balance of resolve. One approach is to simply calculate the balance of resolve similar to how political scientists calculate the balance of capabilities (e.g. Reiter and Stam, 1998b, 380) - each actor's resolve score as a fraction of the total resolve of both actors. However, whereas relative capabilities are measured using a single composite indicator for each side, in an interactionist framework we have two resolve scores for each side, which means we would want to calculate both a relative dispositional resolve score, and a relative situational resolve score. A second approach, and more theoretically interesting, is to adopt a

\footnotetext{
${ }^{6}$ This is especially the case given the extent to which the original experiments focus on public opinion in the context of American foreign policy, such that if one player is playing the United States, the other would be playing a less powerful rival. Herrmann et al. (1997) warn that the full range of images from Herrmann and Fischerkeller's (1995) image theory cannot be tested experimentally on an American sample because of an inability to imagine interacting with a more powerful other.
} 
broader Boolean framework, in which the probability of victory is a function of situational and dispositional resolve for both the great power and the target, in which $\lambda$ once again refers to the inverse logit function $\frac{1}{1+e^{-x}}$ :

$$
\begin{aligned}
\operatorname{Pr}\left(y_{i, t}=1 \mid \alpha, \mathrm{B}, x_{i, t}\right)=\lambda\left(\alpha_{1}+B_{S_{1}} x_{i, t}\right) * \lambda\left(\alpha_{2}+B_{D_{1}} x_{i, t}\right) * & \lambda\left(\alpha_{3}+B_{S_{2}} x_{i, t}\right) \\
& * \lambda\left(\alpha_{4}+B_{D_{2}} x_{i, t}\right)
\end{aligned}
$$

The computationally intensive nature of this setup — with four different paths, two for each actor - thus produces considerable data demands, but has the potential for some intriguing empirical tests. If balance of resolve models are correct, we should see that as a simultaneous increase in each side's situational or dispositional resolve should have no corresponding increase on the probability of victory; it should only be when one side increases its resolve relative to the other that military outcomes should be affected. At the same time, there are interesting questions about logics of substitutability: ceteris paribus, if one side's situational resolve is higher, but the other side's dispositional resolve is higher, what impact does this have on conflict outcomes?

\subsection{The dark side of resolve}

Left unmentioned in the previous chapters is a broader normative issue that is also worth reflecting on. Beneath the massive literature on willpower in the social sciences lies a common normative agenda: a sense that resolve is good. It might seem excessive for two prominent social psychologists to go so far as to suggest that willpower is the "master virtue" (Baumeister and Exline, 1999), but cultural psychologists suggest that self-discipline is a universal value (Schwartz, 1992), and especially in the West, we look down upon the compulsive and weak-willed, caution against "taking the easy way out," laud those who overcome adversity, and treat bounded willpower as a deviation from classical rationality, an acknowledgment of the biases and frailties that characterize ordinary human decision-making 
(Jolls, Sunstein, and Thaler, 1998; Ainslie, 2001). The lionization of resolution reflects two motives. First are political ones, due to self-regulation's relationship with social order. Self-control, after all, is also a form of regulation: Elias's (2000) civilizing processes involve the rise of various norms and customs to regulate impulsive behavior, and Foucauldian analyses of governmentality focus on control of the body and the self, the word "government" referring to self-regulation in a medical or religious sense long before it adopted a legal or political connotation (Lemke, 2001). Second are instrumental ones: we reward motivation out of the belief that the motivated are themselves rewarded, since goals must be fought for in order to be achieved. Indeed, self-control is positively correlated with wealth (Offer, 2006; Ameriks et al., 2007), achievement (Mischel, Shoda, and Rodriguez, 1989) and well-being (Magaletta and Oliver, 1999), and negatively correlated with crime and delinquency (Gottfredson and Hirschi, 1990). It is for this reason that the "libertarian paternalist" tradition in behavioral economics and public policy has begun reflecting on public policy solutions to "nudge" individuals into displaying more self-control, out of the assumption that willpower and welfare go hand in hand (Fennell, 2009). Likewise, when we talk about resolve in IR, we tend to portray it in the same normative light: resolute states are posited to get more at the bargaining table (Morrow, 1985; Powell, 1987) and on the battlefield (Maoz, 1983), while to suggest the public is more resolute than commonly thought is to paint a more favorable picture (Gelpi, Feaver, and Reifler, 2009) than the naysayers.

Yet some of the literature in economics and psychology suggests the potential for what we might call the "dark side of resolve." If the will is a volitional faculty used to initiate action, then it can be employed for negative goals just like positive ones (Kivetz and Simonson, 2002; Fennell, 2009). While myopic decision-making that neglects the future is one type of self-control problem, hyperopic decisionmaking that neglects the present is another (Kivetz and Keinan, 2006). Ainslie (2001, 50-1) notes that excessive self-control leads to "compulsions": excessive dieting can lead to anorexia and excessive attention to detail can border on obsessive 
behavior, while Posner (1997) points to martyr complexes and asceticism as examples of the self-destructive nature of future-oriented selves. A similar phenomenon may be present in IR: some leaders are too indecisive and thus unable to "stay the course," while others are too dogmatic and unable to deviate from it, a "strategic rigidity" that is just as detrimental (Polsky, 2010). An argument could be made that many of the military interventions analyzed in Chapter 5 were not in fact in the national interest of the intervening power. Similarly, Gartzke, Li, and Boehmer (2001) note that there are some methods of signaling resolve that negatively impact your bargaining position, while Powell (1987) suggests that high levels of resolve can lead to perverse outcomes during crisis bargaining, since in order for states to screen out irresolute types, they have to escalate further, thereby rendering crises more likely. If underexpansion (Schweller, 2006) is the product of a lack of political will, overexpansion (Snyder, 1991) might be an indication of too much of it; the "myths of empire" to which Snyder alludes could be understood as the same sorts of "bright line" rules actors make in order to bolster their commitment, which can then prove maladaptive (Schelling, 1984; Ainslie, 2001; Bénabou and Tirole, 2004). In this sense, although this dissertation may be a disquisition on resolve, it should not be mistaken for a veneration of it.

The notion that resolve is neutral with respect to the content of the intentions underlying it means that rather than thinking about resolve exclusively in terms of support for war, future research could also examine resolve not just in nonmilitary domains, but also study resolve in terms of staying out of military misadventures, or studying resolve not just as mere stubbornness, but also reflecting a capacity to adjust. Indeed, although we tend to associate resolve in terms of avoiding the temptation to withdraw - Henry Kissinger famously told Richard Nixon that troop withdrawals were like "salted peanuts" - we can also understand resolve in terms of sailing against the predominant political winds and opposing intervention. It makes little sense to characterize political figures like Senator Wayne Morse of Oregon (a staunch opponent of the War in Vietnam) and Congressman 
Dennis Kucinich of Ohio (an outspoken critic of the invasion of the Iraq) as irresolute because they steadfastly opposed military interventions they never supported in the first place. Similarly, George Washington's Farewell Address argued that as American power grows and foreign policy evolves, the country should avoid the temptation of entangling itself in European affairs - which suggests that one can be resolved about non-intervention just as one can be resolved about pursuing an intervention to victory.

\subsection{On the distinction between situations and dispositions}

One potential concern with the large-N analyses in Chapter 5 is that the leader-level dispositional variables are not in fact measures of the dispositions themselves, but rather, measures of the situational factors that produce them. In the section below, I suggest how the use of text provides one way to get cleaner measures of leaderlevel dispositional traits, but this question also raises a broader concern: namely, if situational variables can be used to proxy dispositional ones, how meaningful is it to distinguish between situational and dispositional variables anyway?

Although the situational-dispositional distinction has a long heritage in social psychology (Heider, 1958; Ross, 1977; Gilbert, 1998) — and mirrors similar dichotomies like the agent-structure debate (Wendt, 1987; Dessler, 1989; Carlsnaes, 1992) - it is worth noting that the situational-dispositional distinction can blur together in at least two ways that have interesting implications for the study of resolve. First, particularly from an thicker interactionist perspective, many factors we consider to be situational features are in fact dispositionally derived. "The stakes" inherent in a situation are laden with subjective (and intersubjective) meaning: protracted conflicts often persist over parcels of land not because of their innate physical characteristics, but because of the meaning with which they've been imbued (Goddard, 2006; Toft, 2006; Hassner, 2006/2007). Similarly, recent studies suggest that some Iranians now see the pursuit of a nuclear program as a sacred value, such that abandoning the program in exchange for material incentives is perceived as taboo (De- 
hghani et al., 2009, 2010), thereby indicating that the stakes of the Iranian nuclear program are not simply a feature of the world, but vary systematically in the minds of Iranians.

Just as some situational factors draw their meaning through dispositional characteristics, many dispositional traits are often assumed to have situational causes. Benjamin, Choi, and Strickland (2010), for example, argue that social and cultural factors shape time preferences, while Wang, Rieger, and Hens (2010) show that economic development is associated with longer $\delta$ parameters. More abstractly, the basic tenet of prospect theory is that risk attitudes depend on a situational feature namely, whether the actor is in the domain of gains or losses (Kahneman and Tversky, 2000). In a similar vein, there is a long tradition of "geodeterminist" arguments that use environmental factors to explain the emergence of particular societal-level characteristics. In the history of political geography, Frederick Jackson Turner's famous frontier thesis, for example, argues that America's wide open spaces and expanding Western frontier were responsible for the individualistic nature of its political culture (Turner, 1956), while Sergey Solovyov, a nineteenth century Russian historian, made similar arguments tracing Russia's unique national character to the flatness and vast expanses of its steppes (Bassin, 1993). In psychology, Van de Vliert et al. (2013) show that climate extremity strongly predicts the distribution of collectivist orientations across Chinese provinces, whereas Schaller and Murray (2008) show that disease prevalence predict regional differences in personality traits. Closer to home, Mead's (2002) argument about the Jacksonian tradition in America - an impulse we can see as being conceptually similar to an honor culture - draws from the work of the historian David Hackett Fischer in tracing Jacksonianism to the influence of the "borderers", immigrants from the Scottish-English and Northern Ireland border area, whose constant exposure to conflict inculcated a marked distrust of those outside of the "folk community." (Fischer, 1989).

There are two speculative implications here that are of interest to theories of resolve like this one. First, if dispositional characteristics are indeed shaped over 
time by broader situational forces, we are likely to see that the distributions of distributional characteristics will vary both within and across populations. Indeed, the premise of cross-cultural psychology is that distributions of dispositional characteristics vary across countries and regions (McCrae and Terracciano, 2005; Rentfrow, Gosling, and Potter, 2008; Bartram, 2013), even if our perceptions of these "national characters" tend to be highly inaccurate (Terracciano et al., 2005). If the distribution of dispositional characteristics vary across populations, and leaders are drawn from these populations, then we might imagine that leader-level dispositional characteristics should also vary across countries as well, even if particular dispositional traits are correlated with the probability of becoming a leader in the first place. Rather than leader-level dispositional characteristics "washing out," we would still expect different distributions to occur.

Second, the relative contribution of dispositional sources of resolve on conflict outcomes should vary over time. If globalization is marked by diffusion processes (Simmons, Dobbin, and Garrett, 2006) and an increasing homogeneity amongst ruling elites, we should expect that these dispositional differences should decrease over time, such that dispositional determinants of resolve would likely have had a greater impact on war outcomes 100 years ago than they will 100 years from now. Similarly, given the extent to which "international society" in the seventeenth and eighteenth centuries consisted of what Morgenthau $(1985,236)$ calls a "small, cohesive, and homogenous" aristocracy, who had received the same education, shared the same set of values, and were often part of the same family, one might imagine there were fewer dispositional differences amongst the European leaders 300 years ago than between present world leaders, such that the leader-level dispositional determinants of resolve should have had less of an impact on military outcomes then than today. ${ }^{7}$ Both of these propositions are purely speculative and beyond

\footnotetext{
${ }^{7}$ This is especially the case if we think some of the dispositional sources of resolve at the leaderlevel have genetic origins. The debate on genopolitcs is vast and far beyond the scope of this footnote (see Fowler and Dawes, 2008; Charney and English, 2012), apart from two points. First, many psychologists understand self-control to be a trait or individual difference (e.g. Muraven et al., 2005), and since behavioral geneticists argue that may traits and individual differences are genet-
} 
the scope of the current data, but they each offer intriguing possibilities for further research.

\subsection{Textual analysis of leaders' speeches}

One theme woven through the previous chapters is the extent to which resolve has a discursive element, as rhetoric is used both to bolster and signal resolve (Fearon, 1994; Baum, 2004; Trager, 2011). ${ }^{8}$ Just as public health and social workers talk about "narratives" and "discourses" of resilience (Ungar, 2004; Lau and van Niekerk, 2011), both pundits and political scientists have noted the extent to which American foreign policy is often wrapped up in a rhetoric of resolve. We see this rhetoric reflected both in commonly invoked arguments about the need for America to maintain its reputation for resolve or else lose its credibility (Press, 2005; Fettweis, 2007) — what Tang (2005) calls the "cult of reputation" - and in the "green lantern" theories of politics referred to in Chapter 1, in which the President of the United States simply has to "add resolve and stir" in order to achieve his or her foreign policy goals. Indeed, resolve is rhetorically banded about with such great frequency that Iyengar and Monten (2008) are able to construct a weekly time

ically determined (Loehlin, 1992; Bouchard, 1994; Alford, Funk, and Hibbing, 2005), it should not be surprising that variations in self-control have been traced to heritable genetic influences as well, and that child levels of self-control are strongly predicted by parental levels of self-control (Boutwell and Beaver, 2010). Second, genetic arguments about willpower or self-control do not assume environmental factors are irrelevant. In the past two decades, a large body of work in epidemiology, behavioral genetics and neuroscience has emerged emphasizing gene-environment interaction, in which the effect of environmental stimuli is conditioned by genetic makeup: rather than positing that genes cause psychiatric disorders, for example, there is growing evidence that pathogens in the environment are responsible for the disorders, but that susceptibility to the pathogens varies by genotype (Caspi and Moffitt, 2006). In the realm of willpower, scholars have found evidence of gene-environment interactions at work as well: individuals who suffer from childhood mistreatment are more likely to display chronic self-control problems as adults if their genetic make-up includes the low-activity version of the monoamine oxidase A (MAOA) gene, for example (KimCohen et al., 2006; Beaver et al., 2010). More broadly, though, if we think leader-level dispositions predict resolve, and we think of dispositional characteristics as being at least partly shaped by genetic makeup, it is not a stretch to imagine that genes can play a role here as well, although disentangling genetic arguments from socialization arguments is likely to be difficult in this context.

${ }^{8}$ For this reason, it is interesting that resolve itself has historically played a larger role in the rationalist oeuvre than the constructivist canon, even though constructivists have historically been the ones to study communicative action and rhetorical contestation (Payne, 2001; Müller, 2004; Bially Mattern, 2005; Krebs and Jackson, 2007). 
series of mentions of resolve by senior American officials in major media outlets during the Iraq War. Similarly, when we think of famously resolute figures like Sir Winston Churchill, we think of the heresthetic component to his resolve, in the role that his rhetoric played in rallying the British troops and people behind him.

The role of rhetoric also suggests a different way to study leader-level resolve than the one employed in Chapter 5, one that circumvents some of the potential objections about the indirect nature of measures of leader-level dispositional sources of resolve. As noted in the previous chapter, there is increasing interest in the role of leaders in international politics, but extant quantitative studies of leaders in IR tend to predominantly focus on leader-level attributes (e.g. age (Horowitz, McDermott, and Stam, 2005), tenure (Chiozza and Goemans, 2004), professional background (Dreher et al., 2009), and education (Besley and ReynalQuerol, 2011)) rather than what leaders actually say. Meanwhile, analysis of leaders' speeches figures prominently in the study of operational codes (Leites, 1951; George, 1969; Walker, Schafer, and Young, 1999; Renshon, 2009) but has typically been idiographic rather than nomothetic, consisting of detailed case studies based on a small number of speeches. Recent developments in automated content analysis and natural language processing (see e.g. Hopkins and King, 2010; Grimmer and King, 2011; Spirling, 2012; King, Pan, and Roberts, 2013) offers an opportunity to bridge this gap. The logic behind supervised learning variants of automated content analysis is to (i) build the corpus of relevant texts, (ii) hand-code a small subset of them based on particular categories of interest, and (iii) employ the subset as a training dataset based on which the remaining texts are coded. The key challenge is not coding leaders' time, risk preferences, and honor orientations, but rather, building the corpus, as no centralized repository of leader speeches currently exists. The advantage of this approach is not only that it would provide finer-grained measures of leader dispositional traits than the indirect measures obtained by measuring the dispositions' situational causes, but that these measures would be time-varying. In this sense, they avoid the challenge raised by Bak and Palmer $(2010,259)$ (and 
the difficulty incurred by the use of the World Values Survey data in Chapter 5) of explaining conflict behavior via static measures of dispositional variables.

\subsection{Against a pure dispositional, and a "garbage can" model of resolve}

As noted in Chapter 2, my theory of resolve relies on a particular ontological claim: that resolve is a state with both situational and dispositional causes. Pure dispositionalists, who conceptualize resolve as an inherent actor characteristic, may therefore object to these arguments: from their perspective, it is no more logically coherent to assert that environmental features make you more or less resolved than to assert that they make you more or less tall. ${ }^{9}$ In this view, it is not that the costs of war affect your resolve, but rather, that actors respond to the costs of war, and resolved actors simply respond differently than irresolute ones.

Against the pure dispositionalists, I view resolve as a state rather than as an identity; in so doing, I borrow a maneuver from the person-situation debate in psychology, which faces a similar ontological conundrum about how to deal with phenomena that appear to have both situational and dispositional causes: "to be resolved", in this operation, is equivalent to "to display resolve", just as "to be angry" is equivalent to "to display anger." This ontological move allows me to advance an interactionist theory of resolve, and understand the traditional dichotomy between moral and material factors in war as reflecting dispositional versus situational sources of resolve rather than resolve versus capabilities, as I argued above. Indeed, I would suggest that a pure dispositionalist theory of resolve leads both to some theoretical conundrums. For example, it seems plausible from a dispositionalist perspective to treat resolve and the costs of fighting as competing explanations, such that actors who persist in a conflict because the costs of fighting are low are not actually resolved. However, as argued many times in the previous chapters, the costs of fighting are not the only costs of war, in that actors also concern themselves with the costs of backing down. If we accept that actors who persist in a

\footnotetext{
${ }^{9}$ The effects of gravity on vertebral compression notwithstanding.
} 
conflict because the costs of fighting are low are not actually resolved, we therefore also need to accept that actors who persist in a conflict because the costs of backing down are high are not actually resolved - which puts us in a strange position, in which actors who fight in order to secure a reputation for resolve are not deemed to be resolved, since they are being motivated by instrumental situational forces rather than intrinsic dispositional ones. Ultimately, though, the question of whether resolve is a state or strictly a trait is an ontological claim, and thus beyond the realm of empirical verification or refutation. Further research could examine how our understanding of the dynamics of resolve change when one begins from a different ontological premise.

Readers who accept my ontological claim that resolve is a state caused by both situational stakes and dispositional traits may nonetheless question the particular stakes and traits I have selected for study here. Indeed, there is perhaps an infinite number of potential dispositional and situational features that could merit study. To pick just a few, there's a large literature on the role that optimism and overconfidence have on both willpower (Magaletta and Oliver, 1999; Watson, Chemers, and Preiser, 2001; Bénabou and Tirole, 2002) and military outcomes (Blainey, 1973; Johnson, Wrangham, and Rosen, 2002; Johnson et al., 2006; Lewin, 2012, but see Fey and Ramsay, 2007), while both Organski $(1968,186)$ and Cline $(1994)$ understand national identity and cultural homogeneity as important sources of national morale. Psychologists studying the "Big 5" personality traits (McCrae and Costa, 1987) would point to conscientiousness as a key predictor of resolve (Roberts et al., 2005; Ameriks et al., 2007; Mondak, 2010, 53), while both IR and public opinion scholars emphasize the role that media and information environments play in affecting how long the public stays on board (Johnson and Tierney, 2006; Berinsky, 2007). The point, then, is not that reputation costs, the interests at stake, time and risk preferences, honor culture and trait self-control are the only predictors of resolve. Rather, the motivation behind the framework is to use prior theoretical expectations to cull a finite number of variables out of a sea of possibilities, out of the 
belief that models in the social sciences are sketches rather than photographs. In this sense, this project is designed not to be the last word on resolve, but an invitation for future conversation; I encourage scholars interested in other such variables to pick up where this work leaves off. 


\section{Appendix A: The Person-Situation Debate}

\section{A.1 Interactionism in action: the person-situation debate}

Personality psychology was constructed on the dispositional premise that individuals displayed stable traits or "individual differences" - the most prominent of which in the contemporary literature are the "Big 5" personality traits (McCrae and Costa, 1987; Mondak, 2010) - that could be used to explain why people often behave strikingly differently from one another (Bauer, 2011). The central challenge for these approaches, however, was how stable traits could be used to explain the surprisingly high level of intraindividual variability evident when individual behavior is studied over time, particularly when the same individuals behaved remarkably differently across different situations (Mischel, 1968). The dynamics of the debate are less important for our purposes than the fact that as Fleeson (2004, 83) notes, it came to a conclusion "because both sides of the debate turned out to be right." Rather than viewing traits as global and situation-free, psychologists began to examine how these dispositional factors display conditional effects based on particular aspects of the environment (Mischel, 2004), and a battery of increasingly sophisticated research designs revealed that although traits are poor at predicting single behaviors, they nonetheless do well at predicting overall trends (Fleeson, 2001).

Figure A.1 borrows from Fleeson's (2001) work, which seeks to reconcile the person-situation debate by viewing traits as "density distributions of states." In this approach, an observer measures an actor's trait-relevant behavior (in our case, the extent to which an actor displays resolve, maintaining a policy despite contrary inclinations or temptations to back down) at repeated intervals. The multiple mea- 
Figure A.1: Resolve as probability density functions
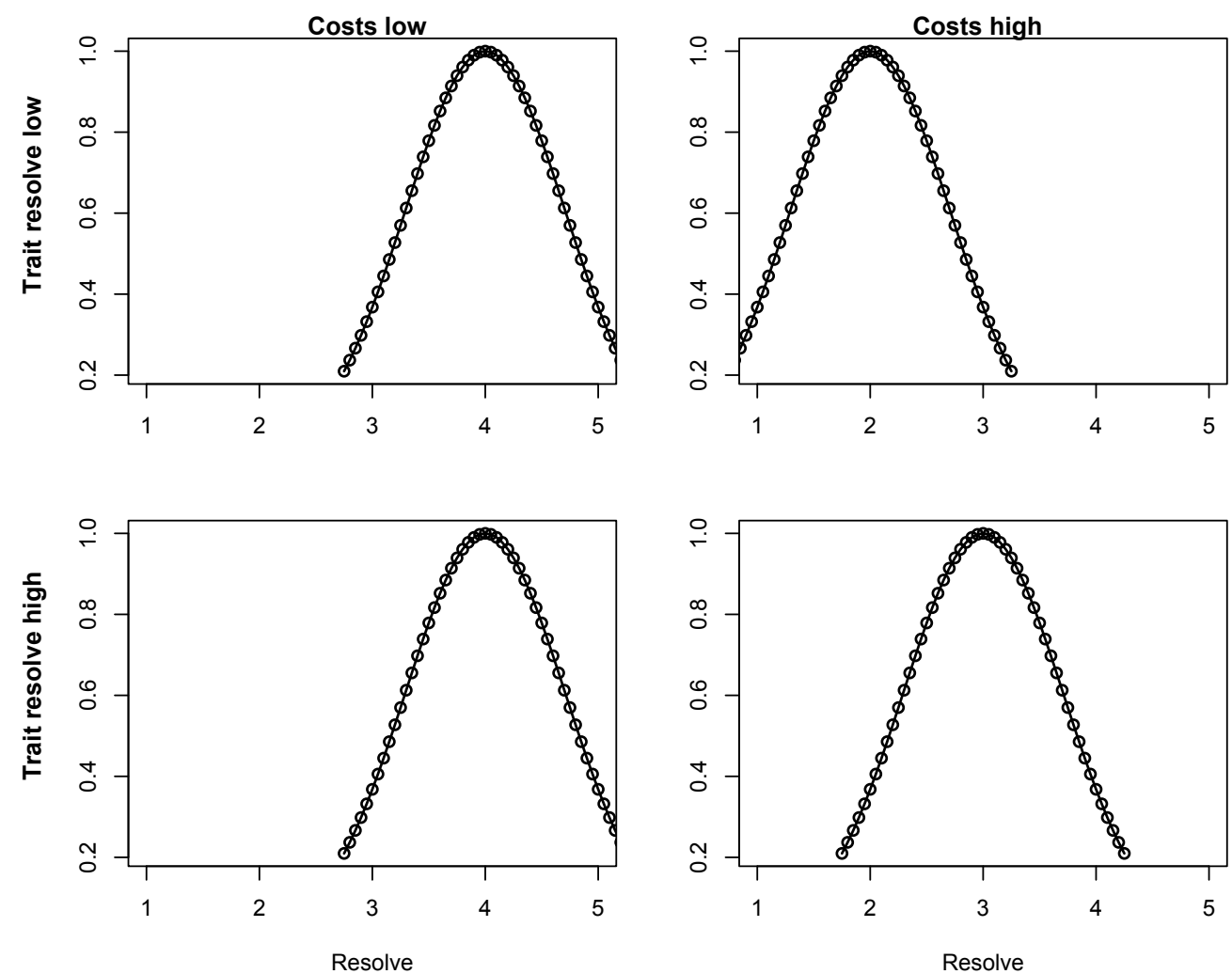

sures produce probability density functions (PDFs), whose moments (mean, variance, and so on) can be used to make inferences about the different distributions of resolve that arise with different types of actors in different types of settings. Thinking about resolve abstractly in this interactionist manner produces the $2 \times 2$ above, in which each of the four panels corresponds to the distribution of resolve manifested in a different dispositional-situational combination, with the x-axes measuring the level of resolve observed at a given moment, and the y-axes indicating the kernel densities of the distributions.

Across the $2 \times 2$ as a whole, the columns illustrate how resolve is affected by situational factors, and the rows by dispositional ones. Thus, the two columns of the figure depict the distribution of resolve when an actor's costs of war are low or high, respectively, while the two rows map out the impact of a dispositional factor, which, borrowing from the psychological literature, I call "trait resolve", to refer to 
an actor's general tendency to maintain her policies despite contrary inclinations or temptations to back down (Tangney, Baumeister, and Boone, 2004). The key conclusion to be drawn from the figure is how when the costs of war are low (the left-hand half of the figure), a pooling equilibrium exists, as both resolute and irresolute "types" display the same level of resolve $(\bar{x}=4)$. When the costs of war are high (the right-hand half), however, a separating equilibrium appears: resolute types will be less resolved than when they are facing lower costs of war $(\bar{x}=3)$, but nonetheless display more resolve than irresolute types, who wilt completely $(\bar{x}=2)$. Notably, this pattern of results differs from those predicted by either the strict dispositional or the purely situational account specified by the endpoints of the dispositional-situational spectrum displayed in Figure 1.2 in Chapter 1. In a purely dispositional account, moving from the left-hand to the right-hand column should not affect the distributions, while in a purely situational account, moving from the top row to the bottom one should have a similarly nonsignificant effect. ${ }^{1}$

Figure A.1 is useful as a preliminary illustration of what testing an interactionist theory looks like, but has three major shortcomings, the first involving its rows, the second implicating its columns, and the third reflecting on the two-dimensional nature of the chart altogether. First, turning to the rows, we need to specify more precisely what the dispositional sources of resolve might be. Figure A.1 uses "trait resolve" as a placeholder, but since traits are defined as "intraindividual consistencies... in propensities to behave" (Tett and Burnett, 2003, 502), we run the risk of a tautology, attempting to explain variation in resolved behavior by simply positing that actors differ in their propensity to display resolve. To produce a satisfactory causal theory - whether interactionist or merely dispositional - we need to specify which attributes of actors make them more likely to exhibit resolve in the first place, and thus to display the patterns in the bottom row of Figure A.1 rather than the top row. Second, turning to the columns, we need to further specify what counts as the costs of war. Since actors engaged in conflict must weigh a number of

\footnotetext{
${ }^{1}$ The plots are oversimplified in that the interactionist literature in psychology looks not just at the means of the distributions, but also at the variance (Fleeson, 2001; Mischel, 2004).
} 
different types of costs (reputational costs, casualties, economic costs, opportunity costs, and so on), it is important to open up the black box of "costliness" and elaborate which types of costs count as costly. Moving from an abstract interactionist framework to an interactionist theory therefore requires us to more precisely stipulate what the dispositional and situational variables in the theory are - a task I attempt in Chapter 2.

Third, although treating situational and dispositional factors as independent dimensions in a figure is a useful heuristic device, if we're interested in pursuing the thickest interactionist theorizing outlined in Table 2.1, the structure of the $2 \times 2$ is somewhat misleading, since it implies the existence of an objective situational reality apart from the observer, when dispositional factors may affect an actor's perception of the environment in the first place. Chapter 2 addresses these three questions, beginning by turning to the issue of costliness, which, once disaggregated, allows for situational features to be incorporated into actors' dispositions. 


\section{Appendix B: Lab Experiment Supplemental Analyses}

\section{B.1 Manipulation checks}

The main analyses in Chapter 3 suggest that the effect of reputation costs differ between participants who initially approved of the intervention and those who did not. There are a number of potential explanations for the divergent effects of the reputation cost manipulation, one stemming from selection effects, the other from escalation effects. Results at the first stage of the intervention scenario suggest that reputation costs increase the likelihood of initially wanting to intervene, especially among individuals who received the dispositional questionnaire prior to being presented with the intervention scenario. If reputation costs are already taken into account ex ante, it is possible that at the second stage they would no longer appear to exert any effect. If this selection effect argument is true, though, it raises the puzzle of why we do not see the same pattern for the anticipated costs treatment, which instead appears to increase in size among participants who supported the initial intervention. Second, it is also possible that reputation costs are automatically rendered salient by intervening: we know that the costs of backing down increase with the level of escalation (Fearon, 1994), so the act of the US deploying troops may raise the costs of backing down sufficiently that the reputation manipulation will no longer exert an effect on its own. Third, it is also possible that perceptions of reputation costs are largely a dispositional phenomenon.

Manipulation checks offer a preliminary means of testing the selection effects hypothesis. After participants withdrew, they were were presented with two ma- 
nipulation checks, one of which asked participants about the reputational costs they thought the US would face for backing down on a scale from 1-5, with 1 indicating minimal costs, and 5 indicating very steep costs. If the selection effects explanation is true, we would expect to see two findings:

1. Individuals who initially wanted to intervene should perceive higher reputation costs for withdrawal than those who wanted to stay out, thereby demonstrating that reputational considerations are associated with the initial decision to intervene.

2. The strength of the reputation cost manipulation should vary based upon participants' initial degrees of support, since pro-intervention respondents should already have been moved by reputational costs, while anti-intervention respondents should not.

We find support for both of these propositions. First, participants who originally supported the intervention were far more likely to believe the US would pay higher reputation costs than those who did not $(\mathrm{M}=3.19$ vs 2.84; Mann-Whitney $\mathrm{U}$ Test: $W=12948, p<0.003)$. Second, the strength of the reputation manipulation varies based on the initial decision to intervene. When respondents are studied as a whole, no significant differences were found between those in the reputation cost condition versus the control condition ( $\mathrm{M}=3.04$ vs 3.13; Mann-Whitney U Test: $W=13362, p<0.324)$, but when the manipulation check is reanalyzed based on whether participants initially supported the mission, the two groups diverge. Amongst participants who supported intervening, the reputation cost manipulation had no effect on the costs participants thought the US would pay for withdrawing ( $\mathrm{M}=3.06$ vs 3.13; Mann-Whitney U Test: $W=6120.5, p<0.736$ ). However, amongst participants who wanted to stay out, the reputation cost manipulation actually has a negative effect, inducing skepticism that the US would pay reputational costs for backing down ( $M=2.90$ vs 3.23; Mann-Whitney U Test: $W=1401.5, p<0.061)$. Note, however, that this latter effect is also consistent with 
a motivated reasoning argument, in which individuals who do not believe that an intervention is worth prosecuting will therefore therefore underweight criteria that could be used to support it.

If the escalation effects argument is true, perceived reputation costs should increase with the level of American commitment, and thus, the longer the mission continues, the steeper the perceived costs of backing down. An ANOVA confirms that the longer participants support the mission, the steeper the reputation costs, as participants who stayed in longer predicted steeper reputational consequences for withdrawal than those who pulled out earlier $(F=32.90, p<0.000)$. Here too, though, we can't distinguish an escalation effects argument from a motivated reasoning one, in which individuals justify their support for an intervention by appealing to the costs of backing down.

Indeed, all of the results with the manipulation checks also support a dispositional explanation, in which reminding participants of reputational costs does not affect their beliefs about what these costs will be, because they already have established beliefs about what the costs of backing down are in the first place. Table B.1 displays the results from an OLS regression in which perceived reputation costs are regressed against the experimental treatments as well as a number of individual difference variables and demographic characteristics. ${ }^{1}$ Although a number of characteristics are associated with higher perceived reputational consequences of backing down - military service, identifying as a strong Republican, and the casualty manipulation - subscribing to a culture of honor has by far the most substantively significant effect, even when controlling for participants' initial desire to intervene. ${ }^{2}$ The third model in the table adds an interaction between honor culture and the reputation manipulation; although the associated $\mathrm{p}$-value $(p<0.143)$ is outside conventional standards of statistical significance, ${ }^{3}$ it offers suggestive

\footnotetext{
${ }^{1}$ Although the dependent variable ranges from 1-5, because a number of participants responded with non-integer values (e.g. assessing the reputation costs as a 2.5 out of 5), I treat the responses as continuous, hence the use of OLS.

${ }^{2}$ The honor orientation scale is described in further detail in the next section.

${ }^{3}$ In a model with just the manipulations, honor orientations, and the reputation cost $\mathrm{x}$ honor
} 
Table B.1: Manipulation check: perceived reputational consequences of backing down

\begin{tabular}{|c|c|c|c|c|c|c|}
\hline & $\beta$ & $\mathrm{p}$ & $\beta$ & $\mathrm{p}$ & $\beta$ & $\mathrm{p}$ \\
\hline Anticipated costs & $\begin{array}{c}0.128 \\
(0.104)\end{array}$ & 0.221 & $\begin{array}{c}0.142 \\
(0.104)\end{array}$ & 0.171 & $\begin{array}{c}0.135 \\
(0.103)\end{array}$ & 0.195 \\
\hline Reputation costs & $\begin{array}{l}-0.103 \\
(0.104)\end{array}$ & 0.323 & $\begin{array}{l}-0.132 \\
(0.104)\end{array}$ & 0.206 & $\begin{array}{l}-1.173 \\
(0.716)\end{array}$ & 0.102 \\
\hline Casualties & $\begin{array}{c}0.233 \\
(0.104)\end{array}$ & 0.026 & $\begin{array}{c}0.246 \\
(0.104)\end{array}$ & 0.019 & $\begin{array}{c}0.255 \\
(0.104)\end{array}$ & 0.014 \\
\hline Order & $\begin{array}{c}0.013 \\
(0.103)\end{array}$ & 0.900 & $\begin{array}{c}0.032 \\
(0.102)\end{array}$ & 0.758 & $\begin{array}{c}0.032 \\
(0.102)\end{array}$ & 0.754 \\
\hline Male & $\begin{array}{l}-0.144 \\
(0.111)\end{array}$ & 0.197 & $\begin{array}{l}-0.153 \\
(0.110)\end{array}$ & 0.166 & $\begin{array}{l}-0.155 \\
(0.110)\end{array}$ & 0.160 \\
\hline Age & $\begin{array}{c}0.003 \\
(0.012)\end{array}$ & 0.833 & $\begin{array}{c}0.002 \\
(0.012)\end{array}$ & 0.885 & $\begin{array}{c}0.001 \\
(0.012)\end{array}$ & 0.937 \\
\hline White & $\begin{array}{l}-0.053 \\
(0.125)\end{array}$ & 0.672 & $\begin{array}{l}-0.055 \\
(0.125)\end{array}$ & 0.658 & $\begin{array}{l}-0.050 \\
(0.124)\end{array}$ & 0.687 \\
\hline Party ID & $\begin{array}{l}-0.487 \\
(0.196)\end{array}$ & 0.014 & $\begin{array}{l}-0.413 \\
(0.198)\end{array}$ & 0.037 & $\begin{array}{l}-0.399 \\
(0.197)\end{array}$ & 0.044 \\
\hline Honor culture & $\begin{array}{c}1.103 \\
(0.490)\end{array}$ & 0.025 & $\begin{array}{c}0.911 \\
(0.494)\end{array}$ & 0.066 & $\begin{array}{c}0.259 \\
(0.663)\end{array}$ & 0.696 \\
\hline Knowledge (International) & $\begin{array}{c}0.075 \\
(0.221)\end{array}$ & 0.734 & $\begin{array}{c}0.130 \\
(0.220)\end{array}$ & 0.555 & $\begin{array}{c}0.146 \\
(0.220)\end{array}$ & 0.507 \\
\hline Military & $\begin{array}{c}0.361 \\
(0.213)\end{array}$ & 0.091 & $\begin{array}{c}0.351 \\
(0.211)\end{array}$ & 0.097 & $\begin{array}{c}0.346 \\
(0.211)\end{array}$ & 0.102 \\
\hline Political Science major & $\begin{array}{l}-0.135 \\
(0.110)\end{array}$ & 0.219 & $\begin{array}{c}-0.25 \\
(0.109)\end{array}$ & 0.253 & $\begin{array}{l}-0.125 \\
(0.109)\end{array}$ & 0.251 \\
\hline Stay out & & & $\begin{array}{l}-0.269 \\
(0.116)\end{array}$ & 0.021 & $\begin{array}{l}-0.271 \\
(0.116)\end{array}$ & \\
\hline $\begin{array}{l}\text { Reputation costs } \\
\text { x Honor culture }\end{array}$ & & & & & $\begin{array}{c}1.367 \\
(0.930)\end{array}$ & 0.143 \\
\hline Intercept & $\begin{array}{c}2.424 \\
(0.538)\end{array}$ & 0.000 & $\begin{array}{c}2.863 \\
(0.567)\end{array}$ & 0.000 & $\begin{array}{c}3.354 \\
(0.657)\end{array}$ & 0.000 \\
\hline
\end{tabular}

OLS coefficients, SEs in parentheses. All variables except age are scaled from 0-1. 
evidence that the lack of significance of the reputation cost manipulation on the manipulation check is due to individuals low in honor orientation responding negatively to the manipulation, while participants high in honor orientation respond positively. In this respect, we see evidence of the third form of interactionist theorizing discussed in Chapter 2, in which dispositional characteristics affect actors' definition of the situation.

Finally, participants were also presented with another manipulation check, in which they were asked to indicate how many casualties they thought the US would sustain if US troops remained for another year. Unlike with the reputation cost manipulation check, the results here are straightforward: participants in the high casualty condition predicted a higher number of casualties than those in the low casualty condition ( $\mathrm{M}=2272.39$ vs. 240 ; Mann-Whitney $\mathrm{U}$ Test: $=591, p<0.000)$.

\section{B.2 Demand characteristics}

Since participants remain in the same casualty treatment over time - that is, the experiment manipulates absolute casualty levels, not casualty trends (Gartner, 2008a) - it is possible that participants become aware of the purpose of the experiment as time goes on, whereupon their behavior would become less informative in later rounds.

This casualty manipulation check offers the opportunity to test for this possibility: if participants are more accurate in their predictions of future casualties as time goes on, it indicates the existence of a potential demand characteristic problem. ${ }^{4}$ Figure B.1 plots how accurate participants' casualty predictions were, as a function of the casualty treatment, and how long they remained in the intervention, as expressed by equation B.1:

orientation interaction, the interaction's level of statistical significance is a slightly more comfortable $p<0.107$.

${ }^{4}$ I am grateful to Scott Althaus for his helpful comments on this point. 


$$
\widehat{y}_{i t}=\log \left(\frac{1}{B-1} \sum_{b=1}^{B} \frac{\left|{\overline{x_{i t}}}^{*}-T_{i}\right|}{T_{i}}\right)
$$

where $B=1500$ bootstraps, $\bar{x}_{i t}^{*}$ the bootstrapped mean casualty prediction in condition $i$ at time $t$, and $T=$ the true level of casualties (72 in the low-casualty condition, 1284 in the high-casualty condition).

As Figure B.1 illustrates, participants' responses to the casualty manipulation check do not become more accurate the longer they stay in the intervention scenario, thereby mitigating concerns about demand characteristics.

Figure B.1: Bootstrapped mean casualty prediction errors

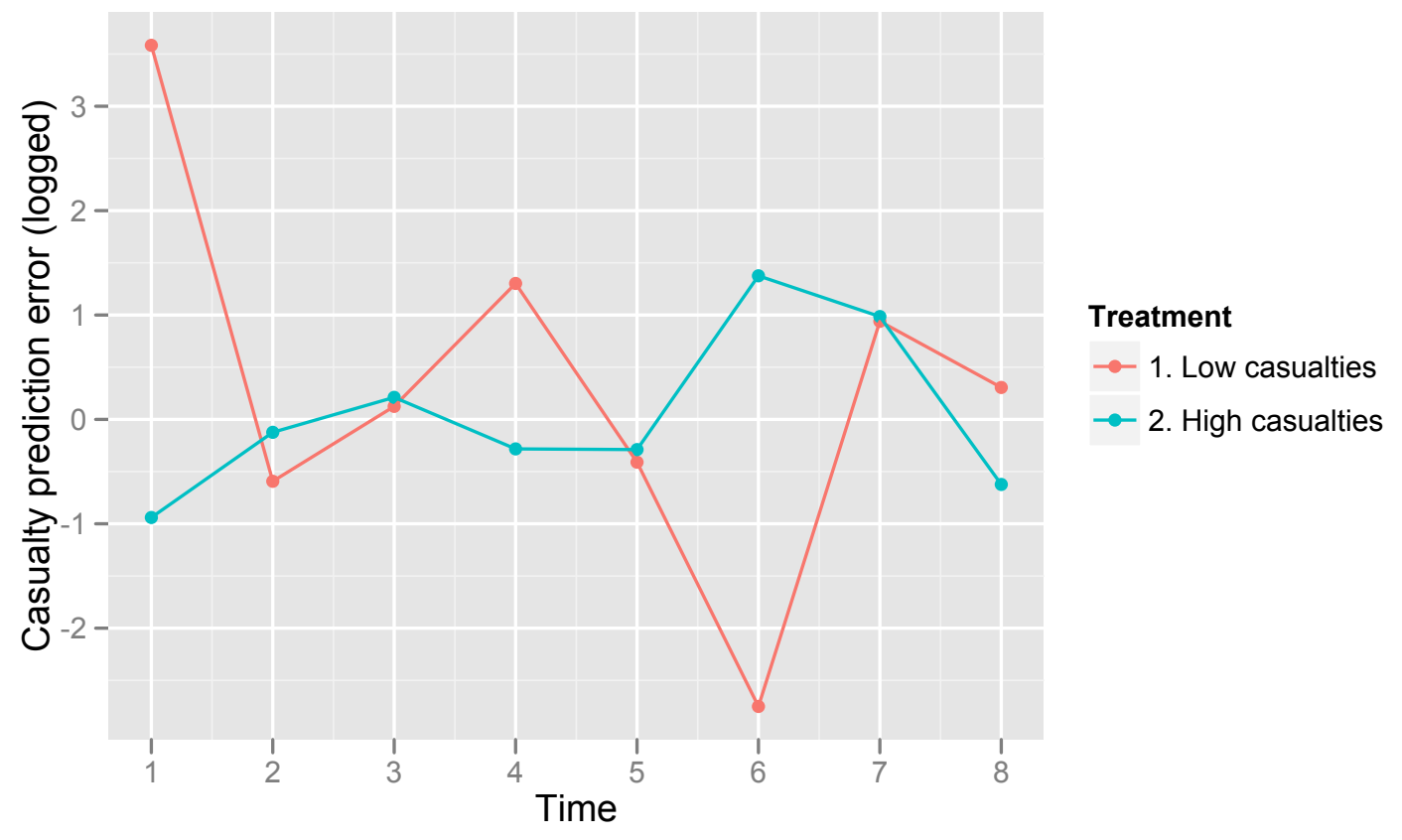

Participants' responses to the casualty manipulation check do not become more accurate the longer they stay in, as measured by the deviation between the bootstrapped mean response to the casualty manipulation check and the casualty treatment level. There is thus little evidence for concerns about demand characteristics.

\section{B.3 Scale reliabilities}

Reliabilities for the scales are shown in Table B.2 below. 
Table B.2: Scale reliabilities

\begin{tabular}{lcc} 
& $\alpha$ & Number of items \\
\hline Honor orientation & 0.511 & 4 \\
Trait self-control: urgency & 0.735 & 4 \\
Trait self-control: perseverance & 0.708 & 4
\end{tabular}

\section{B.4 Demographic controls}

Table B.3 replicates the models estimated in Table 3.4, but also controlling for a number of demographic characteristics like age, race and party ID, and stratifying the baseline hazard on gender. ${ }^{5}$ The results from the first model show that the three main situational treatments retain their statistical significance: anticipated costs boost resolve by $22.7 \%$ and reputation costs by $21.4 \%$, while casualties lower it by $46.5 \%$. Long-term discounting has a similar effect as without the demographic controls, with a one unit increase in $\delta$ associated with a $74.9 \%$ increase in resolve, while present bias continues to lack statistical significance, and is associated with only a $10.9 \%$ change in resolve. Risk aversion's significant curvilinear effect also remains the same, as risk aversion initially decreases resolve (by $28.1 \%$ as $\rho$ grows from 0 to $0.5)$, but gradually increases it again at higher levels of risk aversion $(-53.5 \%$ as $\rho$ increases from 0.5 to 1 ). The one variable that loses its statistical significance because of the addition of demographic controls (particularly gender and party ID) is honor orientation, illustrating the extent to which honor concerns are heavily gendered, and associated with conservative political ideology. ${ }^{6}$ Indeed, it is noteworthy how substantively large an effect partisanship has even when controlling for a number of dispositional variables and situational manipulations: self-described strong

\footnotetext{
${ }^{5}$ Residual-based Proportional Hazard tests indicate that gender's effects violate the proportional hazards assumption, so the models stratify the baseline hazard on gender to avoid biasing the coefficient estimates (see Box-Steffensmeier and Zorn, 2001, 975).

${ }^{6}$ Indeed, the notion of honor is heavily gendered (masculine honor, family honor, feminine honor, etc.), such that the honor literature in cross-cultural psychology typically relies on questions about female sexual purity, male sexual virility, and so on. (See Rodriguez Mosquera, Manstead, and Fischer (2002)). The effect of political ideology is identical to that of party ID: the two variables are highly intercorrelated $(r=0.835)$, so to avoid multicollinearity, I include only party ID in the model.
} 
Republicans were twice as likely to stay in than self-described strong Democrats. Neither race, age, nor the political science major variable were significant.

Table B.3: Demographic characteristics and the duration of the intervention

\begin{tabular}{|c|c|c|c|c|}
\hline & \multicolumn{2}{|c|}{ All participants } & \multicolumn{2}{|c|}{ Pro-intervention participants } \\
\hline & B & Hazard Ratio & B & Hazard Ratio \\
\hline \multirow[t]{2}{*}{ Anticipated costs } & -0.258 & $-22.7 \%$ & -.347 & $-29.3 \%$ \\
\hline & $(0.135)$ & {$[-38.1 \%,-3.5 \%]$} & $(0.167)$ & {$[-46.2 \%,-7.1 \%]$} \\
\hline \multirow{2}{*}{ Reputation costs } & -0.241 & $-21.4 \%$ & -0.222 & $-19.9 \%$ \\
\hline & $(0.138)$ & {$[-37.3 \%,-1.6 \%]$} & $(0.178)$ & {$[-40.2 \%, 7.2 \%]$} \\
\hline \multirow[t]{2}{*}{ Casualties } & 0.382 & $46.5 \%$ & 0.475 & $60.7 \%$ \\
\hline & $(0.138)$ & {$[16.9 \%, 83.7 \%]$} & $(0.175)$ & {$[20.7 \%, 114.1 \%]$} \\
\hline \multirow[t]{2}{*}{ Order } & 0.072 & $7.4 \%$ & 0.218 & $24.4 \%$ \\
\hline & $(0.134)$ & {$[-13.8 \%, 33.9 \%]$} & $(0.174)$ & {$[-6.5 \%, 65.4 \%]$} \\
\hline \multirow[t]{2}{*}{$\delta$} & -1.382 & $-74.9 \%$ & -1.969 & $-86.0 \%$ \\
\hline & $(0.537)$ & {$[-89.6 \%,-39.4 \%]$} & $(0.627)$ & {$[-95.0 \%,-61.0 \%]$} \\
\hline \multirow[t]{2}{*}{$\beta$} & -0.115 & $-10.9 \%$ & -0.306 & $-26.4 \%$ \\
\hline & $(0.171)$ & {$[-32.7 \%, 18.0 \%]$} & $(0.217)$ & {$[-48.4 \%, 5.1 \%]$} \\
\hline \multirow[t]{2}{*}{ Honor culture } & -0.186 & $-17.0 \%$ & 0.643 & $90.3 \%$ \\
\hline & $(0.676)$ & {$[-72.6 \%, 151.4 \%]$} & $(0.850)$ & {$[-53.3 \%, 652.5 \%]$} \\
\hline \multirow[t]{2}{*}{ Risk aversion } & 1.508 & * & 1.430 & * \\
\hline & (1.125) & * & $(1.180)$ & * \\
\hline \multirow[t]{2}{*}{ Risk aversion $^{2}$} & -2.026 & * & -2.418 & * \\
\hline & $(0.915)$ & * & $(1.006)$ & * \\
\hline \multirow[t]{2}{*}{ Party ID } & 1.099 & $200.2 \%$ & 1.442 & $322.9 \%$ \\
\hline & $(0.263)$ & {$[94.9 \%, 362.4 \%]$} & $(0.333)$ & {$[144.9 \%, 630.3 \%]$} \\
\hline \multirow[t]{2}{*}{ Age } & -0.016 & $-1.6 \%$ & -0.002 & $-0.2 \%$ \\
\hline & $(0.017)$ & {$[-4.3 \%, 1.1 \%]$} & $(0.019)$ & {$[-3.2 \%, 2.9 \%]$} \\
\hline \multirow[t]{2}{*}{ White } & -0.165 & $-15.2 \%$ & 0.029 & $2.9 \%$ \\
\hline & $(0.164)$ & {$[-35.2 \%, 11.0 \%]$} & $(0.214)$ & {$[-27.5 \%, 46.2 \%]$} \\
\hline \multirow[t]{2}{*}{ Political Science major } & -0.012 & $-1.2 \%$ & 0.023 & $2.3 \%$ \\
\hline & $(0.139)$ & {$[-21.3 \%, 24.1 \%]$} & $(0.180)$ & {$[-23.8 \%-37.4 \%]$} \\
\hline $\mathrm{N}$ & & 289 & & 199 \\
\hline
\end{tabular}

\section{B.5 Survival curves}

In the main analyses presented in Chapter 3, I visually present the substantive effects using coefficient plots of hazard ratios, since they are relatively straightfor- 
ward to interpret for readers who may be less familiar with event history models. Another way of presenting the results from event history models comes by directly plotting the survival curves, which depicts the probability of the intervention "surviving" - that is, of participants continuing to advocate for the presence of US troops at time $t$; the higher the probability of survival, the more resolve participants display. Figure B.2 plots the survival curves for the situational treatments shown in Table 3.2. Figure B.3 also adds the dispositional variables shown in Table 3.4. Figure B.4 uses survival curves to illustrate the quadratic effect of risk aversion in a different manner to the simulations illustrated by Figure 3.6. Finally, Figures B.5 - B.7 plot survival curves for the interactions between present bias and the casualty treatment (Figure B.5), risk aversion and the reputation cost treatment (Figure B.6), and risk aversion and the casualty treatment (Figure B.7). 
Figure B.2: Survival plots of situational manipulations
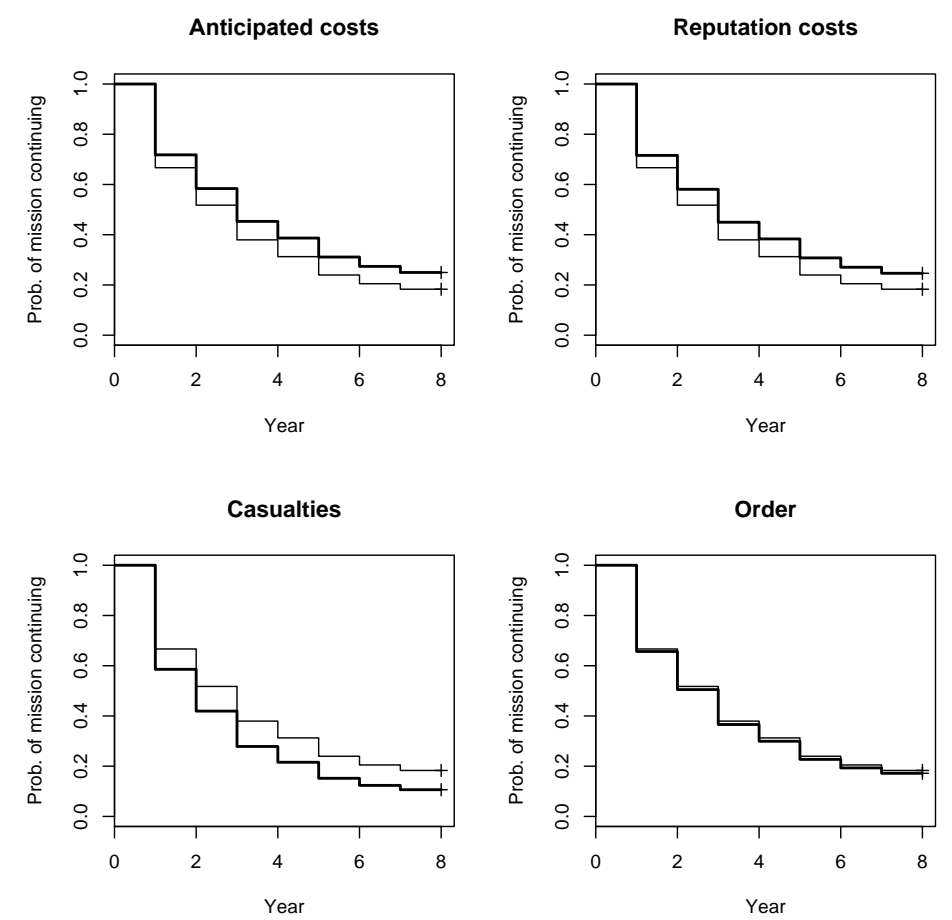

(a) All participants (treatment condition represented by the bold line)
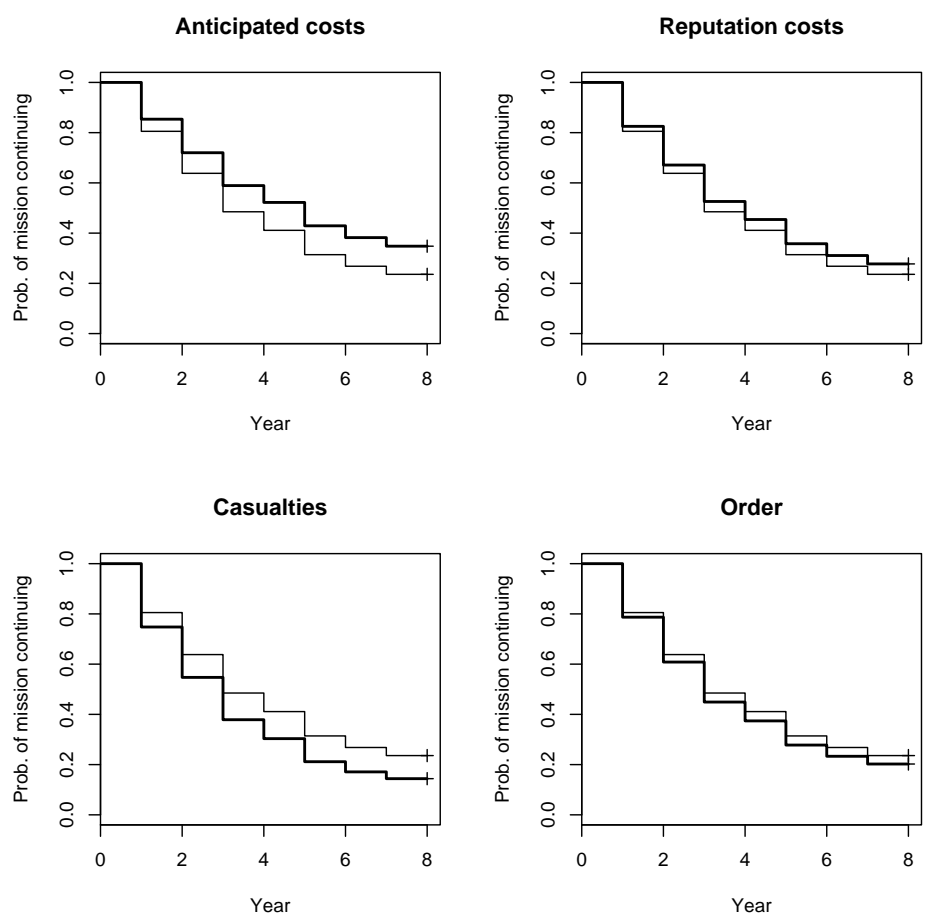

(b) Pro-intervention participants (treatment condition represented by the bold line) 
Figure B.3: Dispositional survival curves
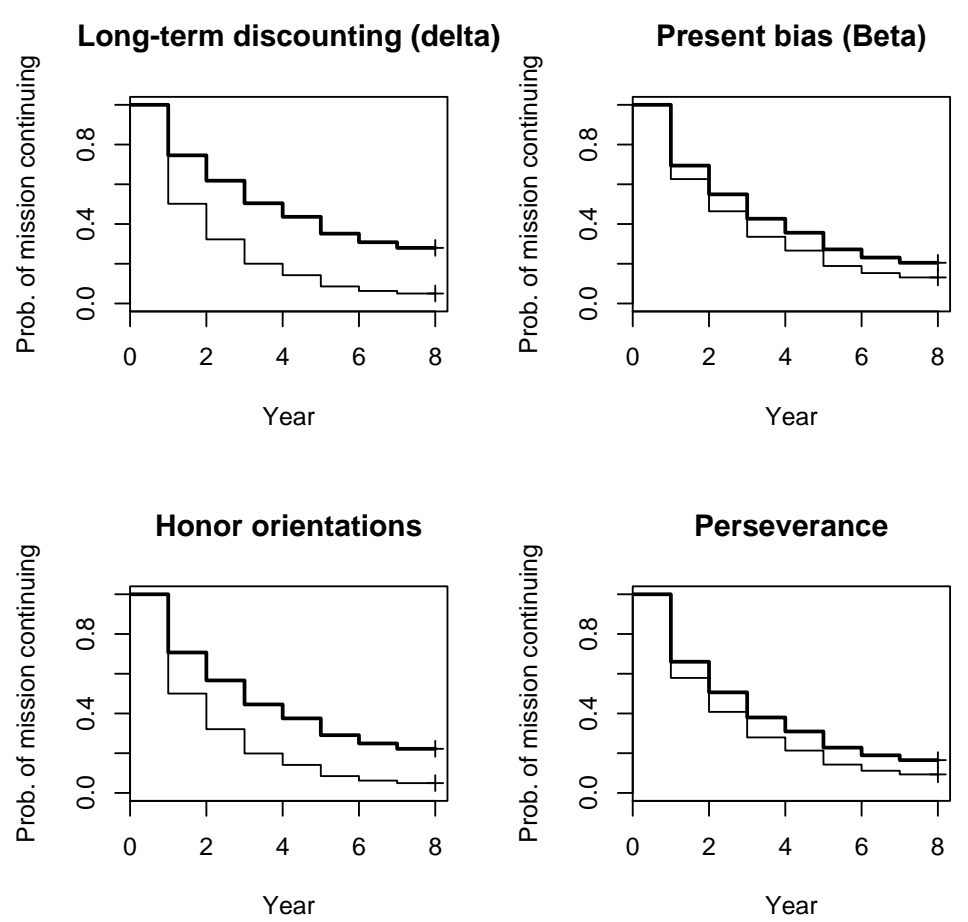

(a) All participants
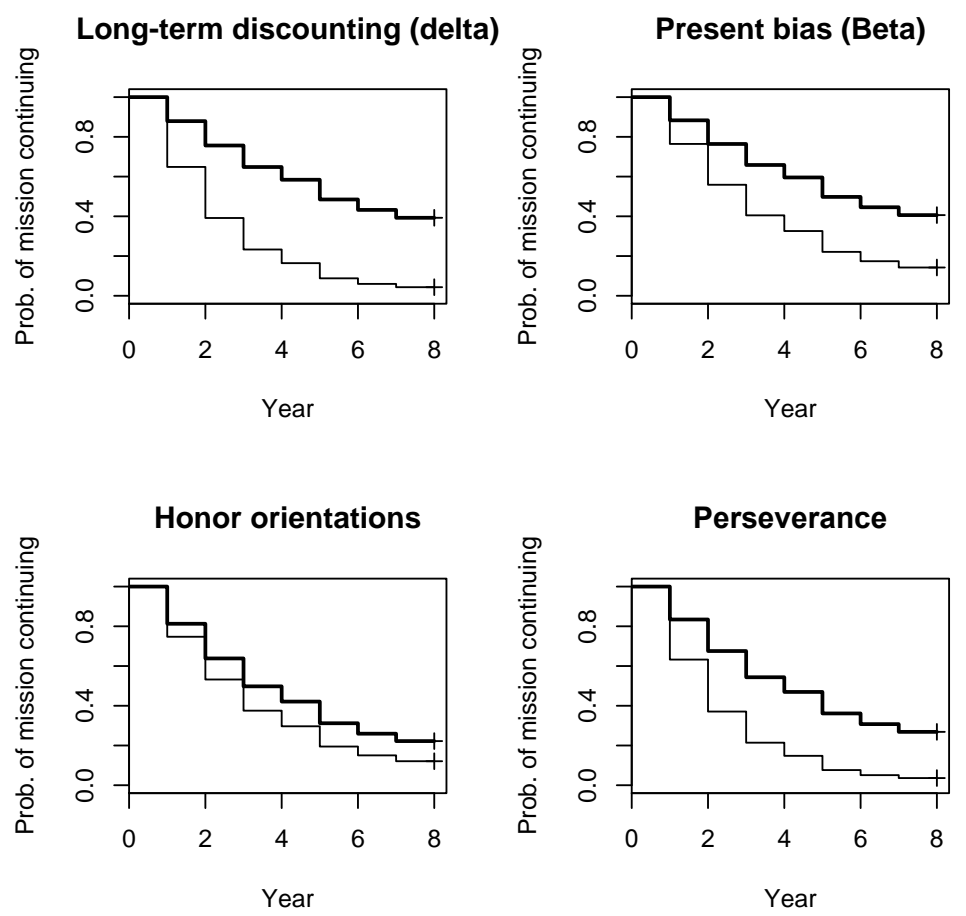

(b) Pro-intervention participants 
Figure B.4: Nonlinear effect of risk preferences survival curves
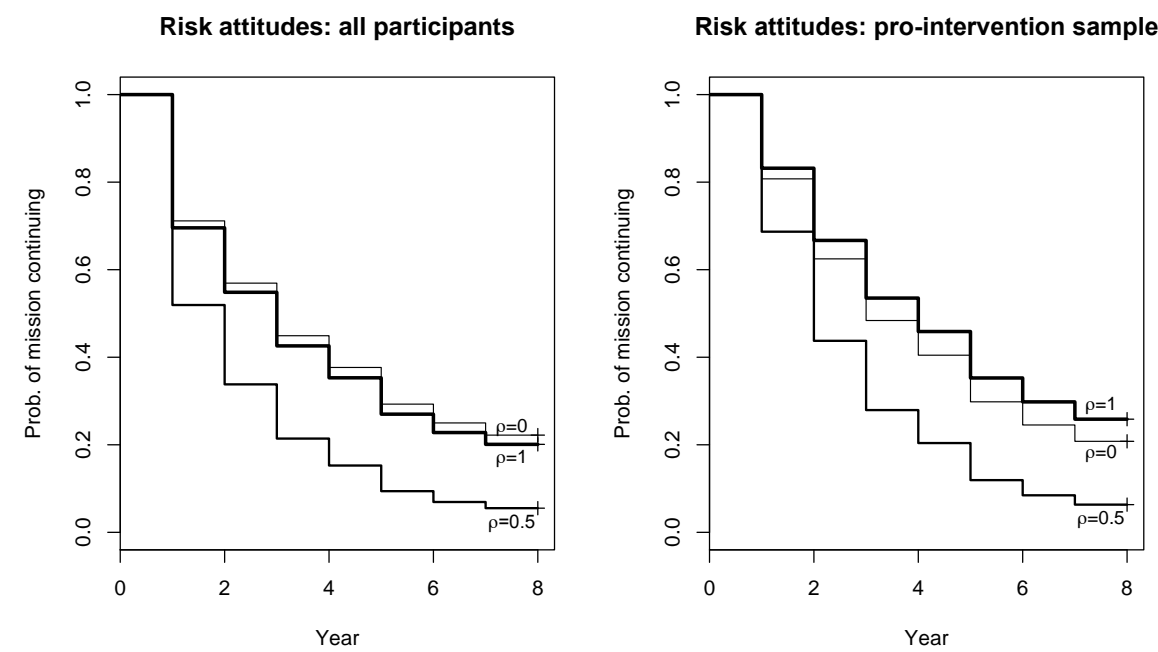

For both the full sample and pro-intervention sub-sample, an increase in risk aversion from risk neutrality $(\rho=0)$ to a moderate level of risk aversion $(\rho=0.5)$ is associated with a large drop in participants' tendency to wait things out; as participants become highly risk averse, however $(\rho=1)$, they become more persistent and less likely to cut and run.

Resolve is thus lowest at moderate levels of risk aversion. 
Figure B.5: Time preferences $x$ casualty survival curves
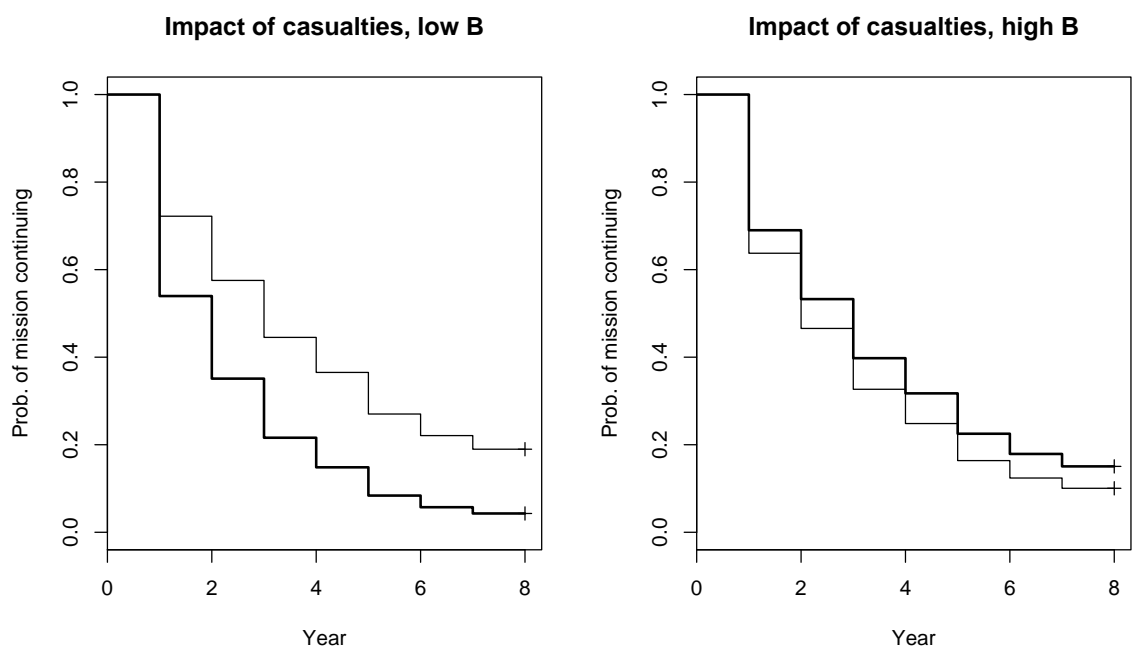

(a) All participants
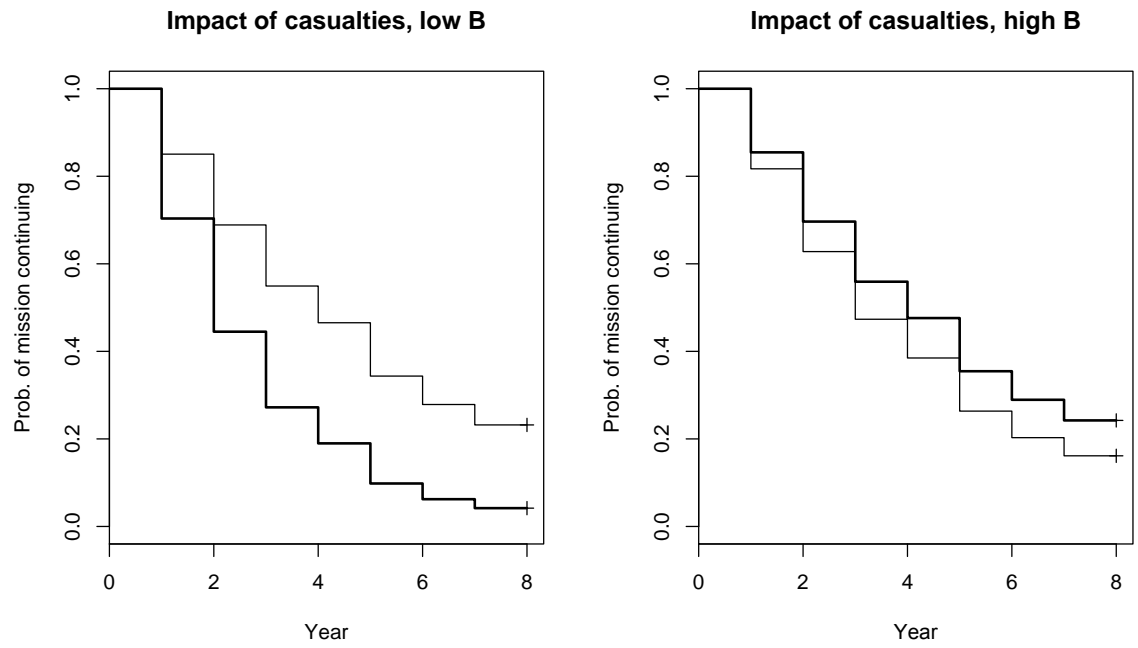

(b) Pro-intervention participants

Individuals with high present bias (low $\beta$ ) are less resolute (more sensitive to casualties) than more patient individuals. Here, low and high values of $\beta$ are set to the 1st and 99th percentiles. 
Figure B.6: Risk preferences x reputation survival curves
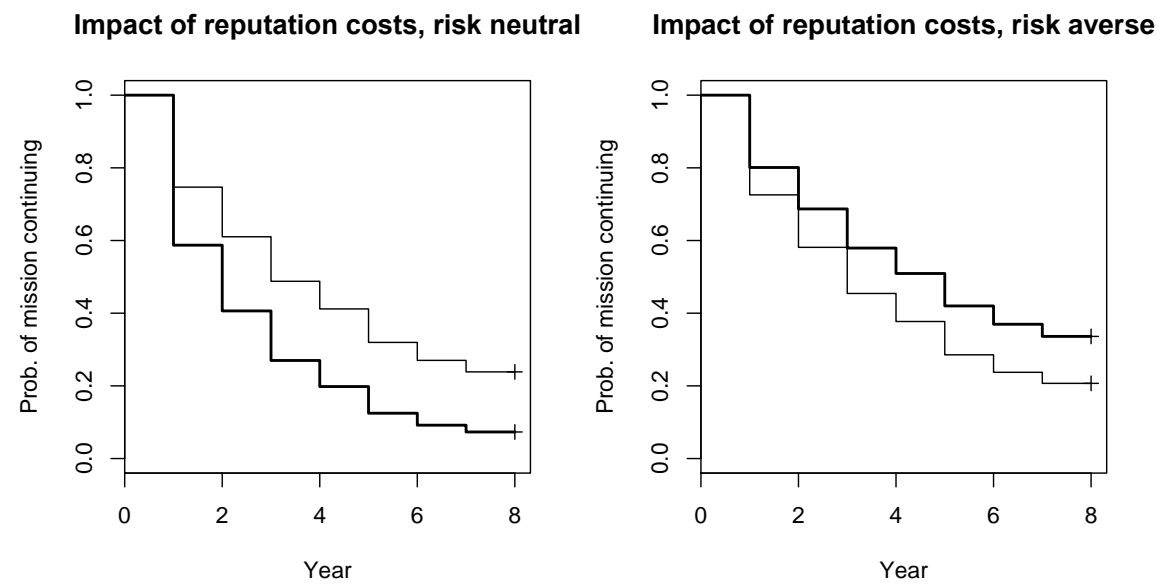

(a) All participants
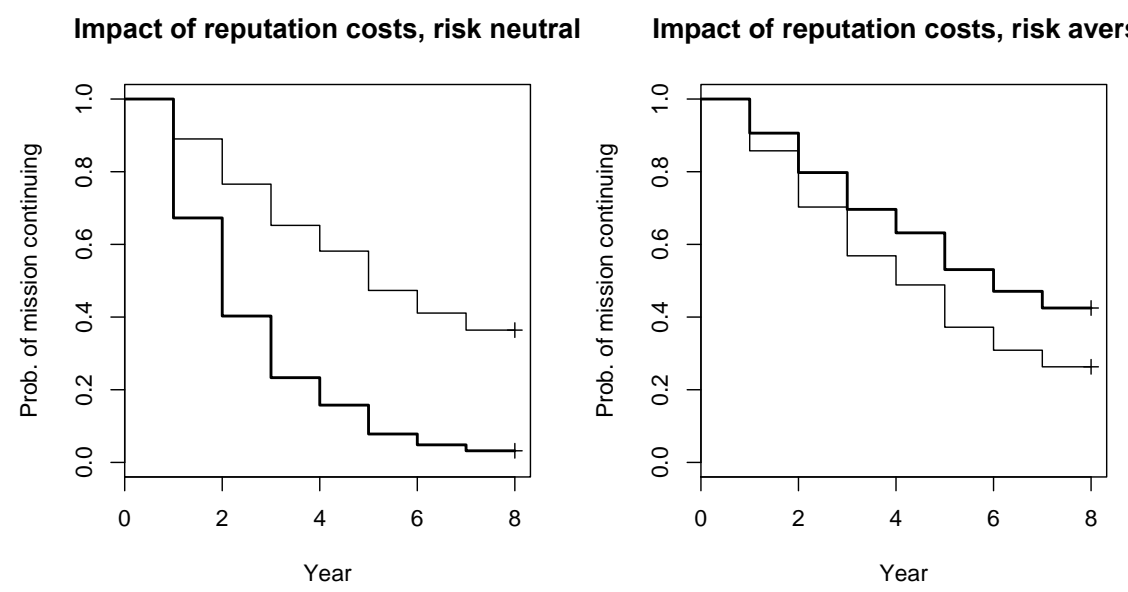

(b) Pro-intervention participants

As risk aversion increases, individuals are more sensitive to reputation costs. 
Figure B.7: Risk preferences x casualty survival curves
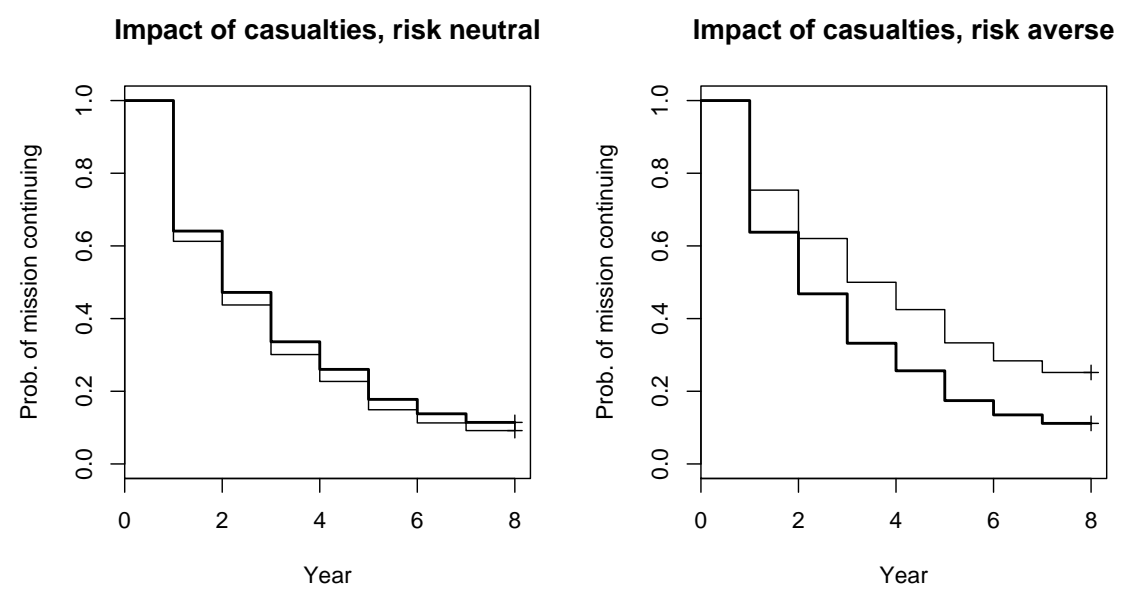

(a) All participants
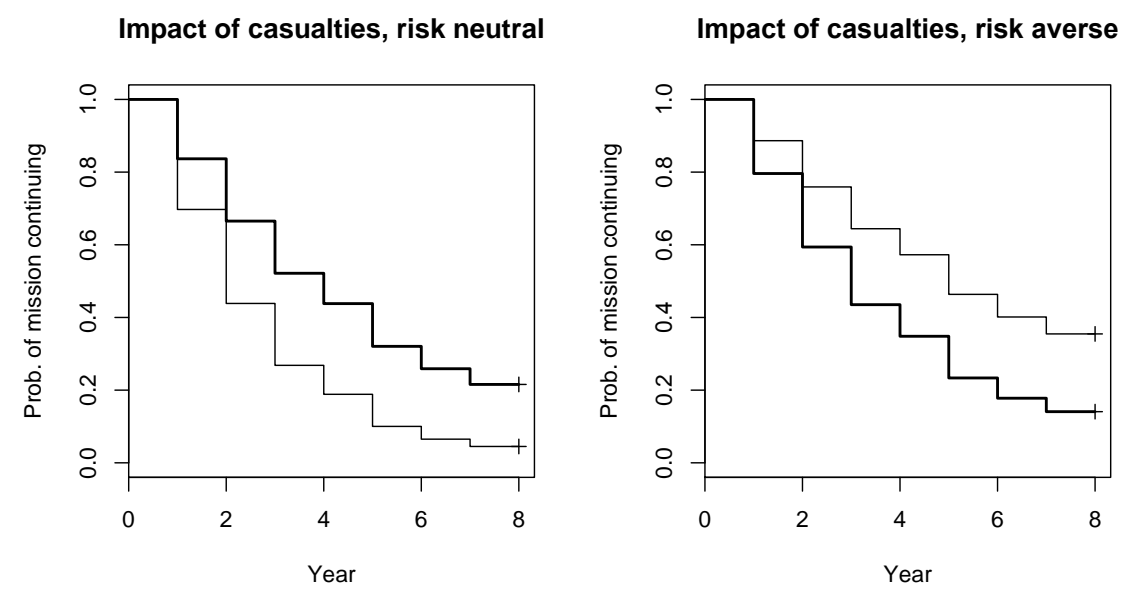

(b) Pro-intervention participants

As risk aversion increases, individuals are more sensitive to casualties. 


\section{B.6 Results from fully-crossed factorial analyses}

Table B.4: Hazard ratios of duration of intervention: full factorial model

\begin{tabular}{|c|c|c|c|c|}
\hline & \multicolumn{4}{|c|}{ Impact of A: (Higher anticipated costs) } \\
\hline & \multicolumn{2}{|c|}{$\mathrm{D}=0$} & \multicolumn{2}{|c|}{$\mathrm{D}=1$} \\
\hline & $\mathrm{B}=0$ & $\mathrm{~B}=1$ & $\mathrm{~B}=0$ & $\mathrm{~B}=1$ \\
\hline \multirow[t]{2}{*}{$\mathrm{C}=0$} & $+16.95 \%$ & $-20.05 \%$ & $-50.05 \%$ ** & $-50.58 \%$ ** \\
\hline & {$[-35.8 \%-131.5 \%]$} & {$[-60.1 \%-26.8 \%]$} & {$[-71.4 \%--12.8 \%]$} & {$[-76.7 \%-4.7 \%]$} \\
\hline \multirow[t]{5}{*}{$\mathrm{C}=1$} & $+1.93 \%$ & $-2.94 \%$ & $-28.68 \%$ & $-13.39 \%$ \\
\hline & {$[-43.2 \%-82.8 \%]$} & {$[-45.9 \%-74.1 \%]$} & {$[-65.9 \%-49.2 \%]$} & {$[-48.5 \%-45.6 \%]$} \\
\hline & \multicolumn{4}{|c|}{ Impact of B: (Reputation costs) } \\
\hline & \multicolumn{2}{|c|}{$\mathrm{D}=0$} & \multicolumn{2}{|c|}{$\mathrm{D}=1$} \\
\hline & $\mathrm{A}=0$ & $\mathrm{~A}=1$ & $\mathrm{~A}=0$ & $\mathrm{~A}=1$ \\
\hline \multirow[t]{2}{*}{$\mathrm{C}=0$} & $+40.58 \%$ & $-3.9 \%$ & $-47.12 \%$ ** & $-47.68 \%$ ** \\
\hline & {$[-21.1 \%-150.6 \%]$} & {$[-46.2 \%-71.6 \%]$} & {$[-70.0 \%--6.7 \%]$} & {$[-75.1 \%-10.1 \%]$} \\
\hline \multirow[t]{5}{*}{$\mathrm{C}=1$} & $+1.49 \%$ & $-3.36 \%$ & $-43.72 \%$ & $-31.65 \%$ \\
\hline & {$[-44.9 \%-87.6 \%]$} & {$[-44.6 \%-68.5 \%]$} & {$[-72.8 \%-16.4 \%]$} & {$[-60.0 \%--5.1 \%]$} \\
\hline & \multicolumn{4}{|c|}{ Impact of C: (Casualties) } \\
\hline & \multicolumn{2}{|c|}{$\mathrm{D}=0$} & \multicolumn{2}{|c|}{$\mathrm{D}=1$} \\
\hline & $\mathrm{A}=0$ & $\mathrm{~A}=1$ & $\mathrm{~A}=0$ & $\mathrm{~A}=1$ \\
\hline \multirow[t]{2}{*}{$\mathrm{B}=0$} & $+33.76 \%$ & $+16.58 \%$ & $+30.72 \%$ & $+86.6 \% *$ \\
\hline & {$[-29.9 \%-155.1 \%]$} & {$[-31.6 \%-98.9 \%]$} & {$[-36.7 \%-170.0 \%]$} & {$[5.4 \%-230.6 \%]$} \\
\hline \multirow[t]{4}{*}{$\mathrm{B}=1$} & $-3.43 \%$ & $+17.24 \%$ & $+39.14 \%$ & $+143.8 \%$ ** \\
\hline & {$[-43.7 \%-65.6 \%]$} & {$[-35.6 \%-113.7 \%]$} & {$[-21.1 \%-145.2 \%]$} & {$[19.1 \%-399.2 \%]$} \\
\hline & \multicolumn{4}{|c|}{ Impact of D: (Order manipulation) } \\
\hline & \multicolumn{2}{|c|}{$\mathrm{B}=0$} & \multicolumn{2}{|c|}{$\mathrm{B}=1$} \\
\hline \multirow{3}{*}{$\mathrm{C}=0$} & $\mathrm{~A}=0$ & $\mathrm{~A}=1$ & $\mathrm{~A}=0$ & $\mathrm{~A}=1$ \\
\hline & $+93.6 \% *$ & $-17.31 \%$ & $-27.18 \%$ & $-54.99 \%$ * \\
\hline & {$[8.6 \%-245 \%]$} & {$[-53.7 \%-47.7 \%]$} & {$[-58.6 \%-28.4 \%]$} & {$[-78.6 \%--5.33 \%]$} \\
\hline \multirow[t]{2}{*}{$\mathrm{C}=1$} & $+89.2 \% \dagger$ & $+32.37 \%$ & $+4.93 \%$ & $-6.38 \%$ \\
\hline & {$[-13.3 \%-313.1 \%]$} & {$[-21.7 \%-123.8 \%]$} & {$[-38.8 \%-80.0 \%]$} & {$[-46.8 \%-64.9 \%]$} \\
\hline
\end{tabular}

$90 \%$ CIs around hazard ratios in brackets. Positive values means more likely to withdraw. 
Figure B.8: Hazard ratio plots: full factorial analysis

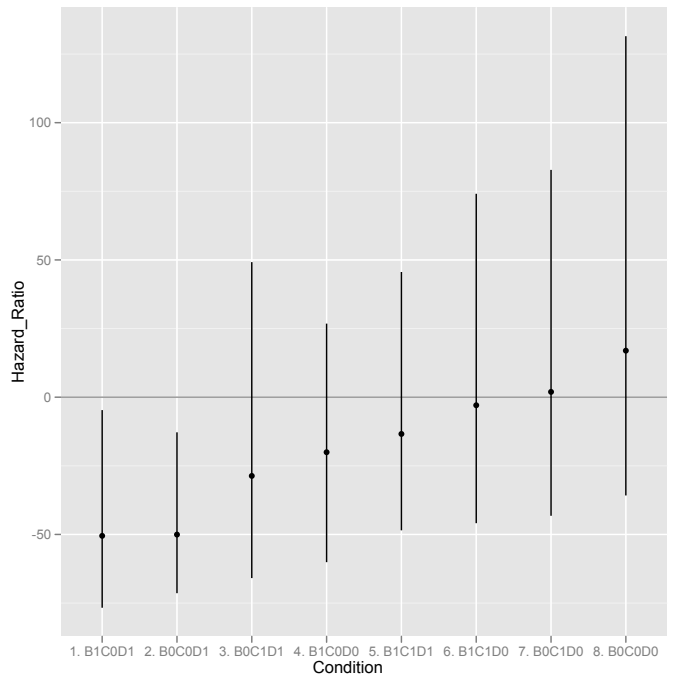

(a) Anticipated costs

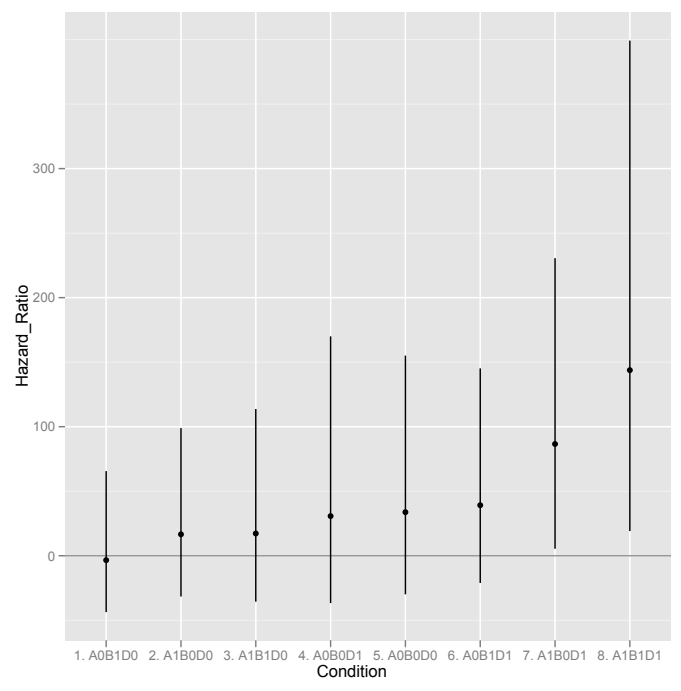

(c) Casualties

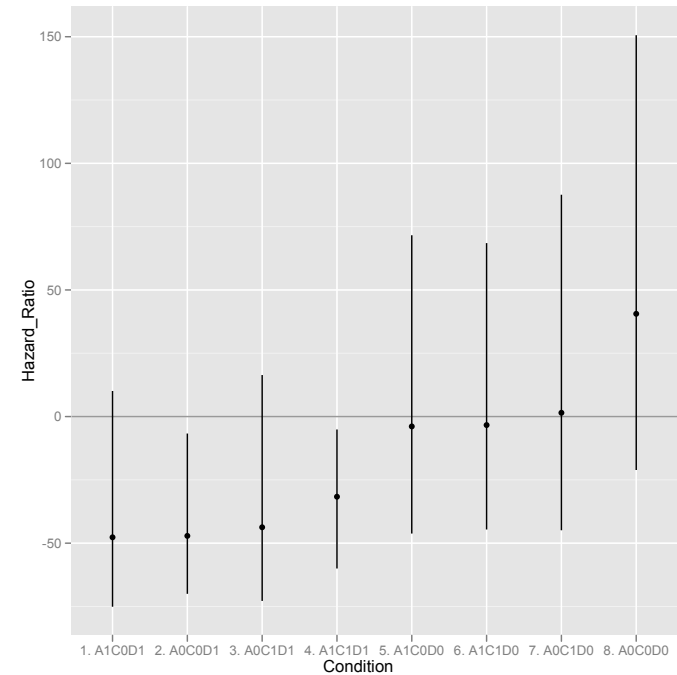

(b) Reputation costs

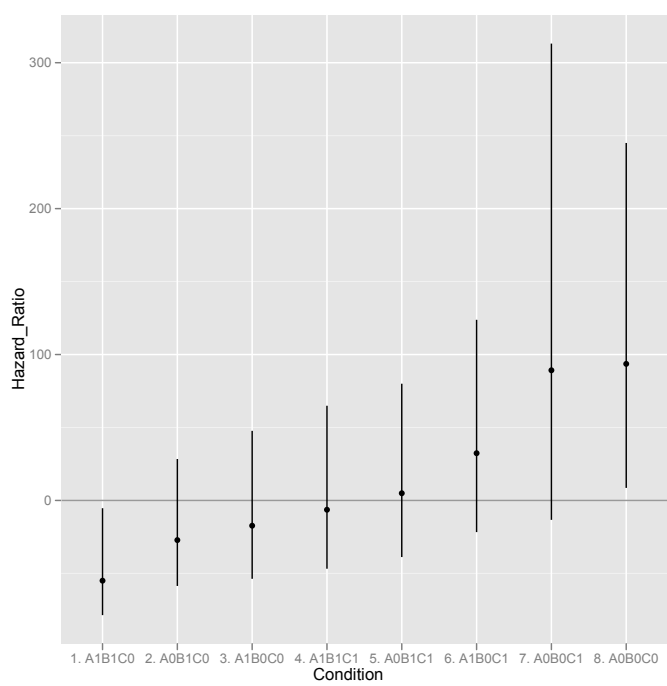

(d) Order manipulation

Hazard ratios of duration of intervention: full factorial model. Because the cell sizes are smaller, the confidence intervals around the hazard ratios are far larger when the experiment is reanalyzed with four-way interactions, but the general pattern remains the same: higher anticipated costs tend to prolong the intervention and make withdrawal less likely, as does rendering reputation costs salient. Casualties, though, have the opposite effect, making interventions more likely to end. In general, respondents were more sensitive to all three of the situational manipulations when they received the dispositional questionnaire first, possibly suggesting that participants took some time to "warm up." 


\section{Appendix C: Survey Experiment Supplementary Analyses}

\section{C.1 Treatment heterogeneity checks}

To ensure that the nonsignificant casualty treatment effect was not obscuring treatment effect heterogeneity, a series of models were estimated with interactions between the casualty treatment and the demographic characteristics for which the survey experiment sample differs the most from the convenience sample used in the lab experiment. Table C.1 depicts the results from a model with interactions between the casualty treatment and age, disaggregated into a system of dummy variables to avoid linearity assumptions. ${ }^{1}$ As the p-values in the third column show, there is no evidence that participants in different age cohorts respond differently to the casualty treatment. Since we have specific theoretical reasons to expect that college-age respondents will behave differently from older participants, age was also dichotomized and separate models estimated featuring an interaction between the treatment and whether participants were 24 or below, but the interaction effect remained insignificant $(p<0.473)$. Similarly, Table C.2 finds no significant interactions between the treatment and education, while Table C.3 finds no significant interactions with geographic region, and Table C.4 finds no significant interactions with income. Likewise, a model estimating an interaction between the casualty treatment and gender finds the treatment's impact is not conditional on gender $(p<0.373)$. The only demographic characteristic where treatment heterogeneity

\footnotetext{
${ }^{1}$ For Tables C.1-C.5, the p-values reported are for the interaction between the treatment and a dummy variable; the baseline categories are listed at the bottom of each table, along with the treatment's significance and percentage change in the hazard rate for that particular group.
} 
is evident is with respect to ethnicity (Table C.5), where Hispanics respond to the casualty treatment by becoming $38.1 \%$ less likely to withdraw at any given point in time. The relatively small proportion of Hispanics in both the lab and the survey experiment, however suggest that this difference is unlikely to be driving the results.

Table C.1: The impact of the casualty treatment is not conditional upon age

\begin{tabular}{ccc}
\hline $\begin{array}{c}\text { Age } \\
\text { Cohort }\end{array}$ & HR & $\begin{array}{c}\text { Casualty treatment effect } \\
\text { p-value from interaction }\end{array}$ \\
\hline $25-34$ & $-3.93 \%$ & $p<0.66$ \\
$35-44$ & $-1.56 \%$ & $p<0.72$ \\
$45-54$ & $-7.75 \%$ & $p<0.53$ \\
$55-64$ & $-2.35 \%$ & $p<0.68$ \\
$65-74$ & $-12.6 \%$ & $p<0.42$ \\
$75+$ & $-30.5 \%$ & $p<0.18$ \\
\hline \multicolumn{3}{c}{ Positive HRs = greater probability of withdrawal. } \\
Baseline category: age cohort 18-24 (HR 7.92\%, $p<0.71$ )
\end{tabular}

Table C.2: The impact of the casualty treatment is not conditional upon education

\begin{tabular}{ccc}
\hline Education & \multicolumn{2}{c}{ Casualty treatment effect } \\
Level & HR & p-value from interaction \\
\hline Less than High School & $16.55 \%$ & $p<0.33$ \\
High School & $-14.43 \%$ & $p<0.59$ \\
Some College & $-5.96 \%$ & $p<0.96$ \\
\hline Positive HRs = greater probability of withdrawal. \\
Baseline category: college graduate (HR $6.72 \%, p<0.53)$
\end{tabular}

\section{C.2 Estimating the casualty treatment effect for compliers}

Experimentalists often have to wrestle with problems of noncompliance, in which participants fail to comply with the experimenter's manipulation (Morton and Williams, 2010, 48). For example, in voter turnout field experiments, some voters may be assigned a treatment (e.g. being visited by a canvasser, or receiving a phone call), but not be home to answer the door (Imai, 2005). However, we can also think of 
Table C.3: The impact of the casualty treatment is not conditional upon geographic region

\begin{tabular}{ccc}
\hline Region & \multicolumn{2}{c}{ Casualty treatment effect } \\
& HR & p-value from interaction \\
\hline Northeast & $-9.81 \%$ & $p<0.39$ \\
South & $-14.93 \%$ & $p<0.17$ \\
West & $-4.29 \%$ & $p<0.56$ \\
\hline Positive HRs = greater probability of withdrawal. \\
Baseline category: Midwest (HR 6.54\%, $p<0.62$ )
\end{tabular}

Table C.4: The impact of the casualty treatment is not conditional upon income

\begin{tabular}{ccc}
\hline Income & \multicolumn{2}{c}{ Casualty treatment effect } \\
Level & HR & p-value from interaction \\
\hline$\$ 20,000-\$ 39,999$ & $3.03 \%$ & $p<0.60$ \\
$\$ 40,000-\$ 59,999$ & $-1.17 \%$ & $p<0.74$ \\
$\$ 60,000-\$ 84,999$ & $-18.35 \%$ & $p<0.64$ \\
$\$ 85,000-\$ 99,999$ & $44.65 \%$ & $p<0.12$ \\
$\$ 100,000-\$ 124,999$ & $-11.13 \%$ & $p<0.92$ \\
$\$ 125,000-\$ 149,999$ & $4.17 \%$ & $p<0.67$ \\
$\$ 150,000-\$ 174,999$ & $-44.24 \%$ & $p<0.21$ \\
$\$ 175,000+$ & $-34.45 \%$ & $p<0.32$ \\
\hline
\end{tabular}

Positive HRs $=$ greater probability of withdrawal.

Baseline category: Less than $\$ 20,000$ (HR $-8.73 \%, p<0.62$ )

Table C.5: The impact of the casualty treatment is conditional upon ethnicity

\begin{tabular}{|c|c|c|}
\hline \multirow{2}{*}{ Ethnicity } & \multicolumn{2}{|c|}{ Casualty treatment effect } \\
\hline & HR & $\mathrm{p}$-value from interaction \\
\hline Black & $9.56 \%$ & $p<0.56$ \\
\hline Hispanic & $-38.10 \%$ & $p<0.04$ \\
\hline Other & $-18.89 \%$ & $p<0.53$ \\
\hline
\end{tabular}

Positive HRs $=$ greater probability of withdrawal.

Baseline category: White, Non-Hispanic (HR $-3.10 \%, p<0.66)$ 
noncompliance as occurring when participants in lab or survey experiments fail to pay sufficient attention to stimuli (Morton and Williams, 2010, 156). One way of gauging the impact of the casualty treatment is thus to use the manipulation check to identify the subset of participants who were the most attentive to the treatment, and estimate the treatment effect for just these compliers.

Figure C.1: Casualty treatment p-values for compliers

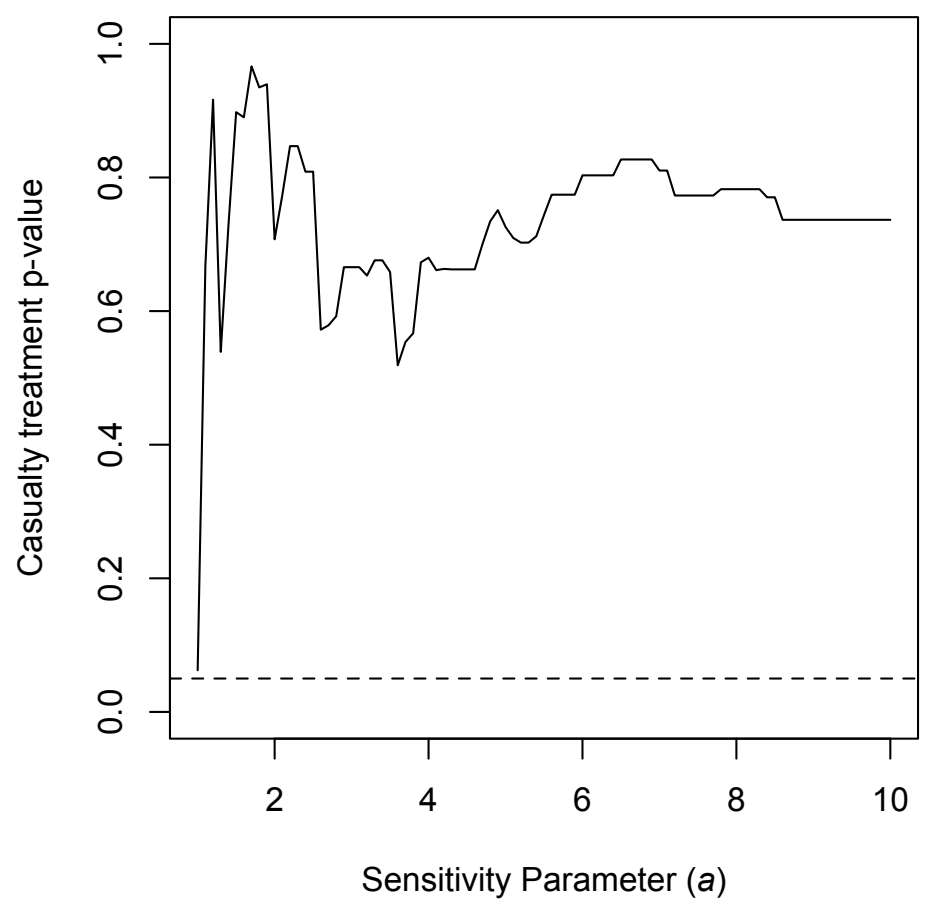

The casualty treatment lacks statistical significance amongst compliers (defined as those participants whose response to the casualty manipulation check ranged from $X_{i} / a$ to $X_{i}(a)$, where $X_{i}$ is the true number of casualties from manipulation $T_{i}$, and $a$ is a sensitivity parameter ranging from 1-10.) The one exception is when $a=1$, but as discussed in the text, the sample size is too small here to draw valid inferences. The dashed line indicates $p<0.05$.

The challenge, however, is determining who counts as a complier. I employ the following approach. First, note that each individual $i$ either receives the highcasualty treatment $\left(T_{i}=1\right)$ in which the US experiences $X_{0}=72$ casualties, or the low-casualty treatment $\left(T_{i}=0\right)$, in which the US experiences $X_{1}=1284$ casualties. Participants also complete a casualty manipulation check, where they indicate $Z_{i}$, the number of casualties they expect the US to receive over the next year. To de- 
termine the thresholds for who counts as a complier, I re-estimate the first model from Table 4.1, but on the subsample of participants for whom $X_{i} / a \leq Z_{i} \leq X_{i}(a)$, where $a=1 \ldots 10$ is a sensitivity parameter; thus, if $a=3$, the group of compliers is defined as those participants in the control group whose manipulation check results range from 24-216, and those participants in the treatment group whose manipulation check results range from 428-3852.

Figure C.1 plots the p-value for the casualty treatment for the compliers, varying $a$ from 1-10. The plot shows that the casualty treatment consistently lacks statistical significance, regardless of the level of $a$, except for when $a=1$ - such that compliers are defined as those participants for whom $X_{i}=Z_{i}$ (that is, they predicted an identical number of casualties as the number of casualties the US received in the previous year). Since this stringent threshold reduces the sample size to 25, however (22 participants in the control group and 3 participants in the treatment group), the analysis is severely underpowered, and as soon as the the sensitivity parameter increases to 1.1 , the $\mathrm{p}$-value jumps back up to $p<0.667$. In other words, it therefore appears that the casualty treatment lacks significance in the survey experiment even amongst compliers, regardless of whether the parameters defining compliance are relatively strict or relatively liberal. 


\section{Appendix D: Boolean Supplementary Analyses}

D.1 Alternative bootstrapped boolean coefficient estimates

Figures D.1 - D.5, below, illustrate the bootstrapped Boolean coefficient estimates for models 1-5 from Table 5.3. 


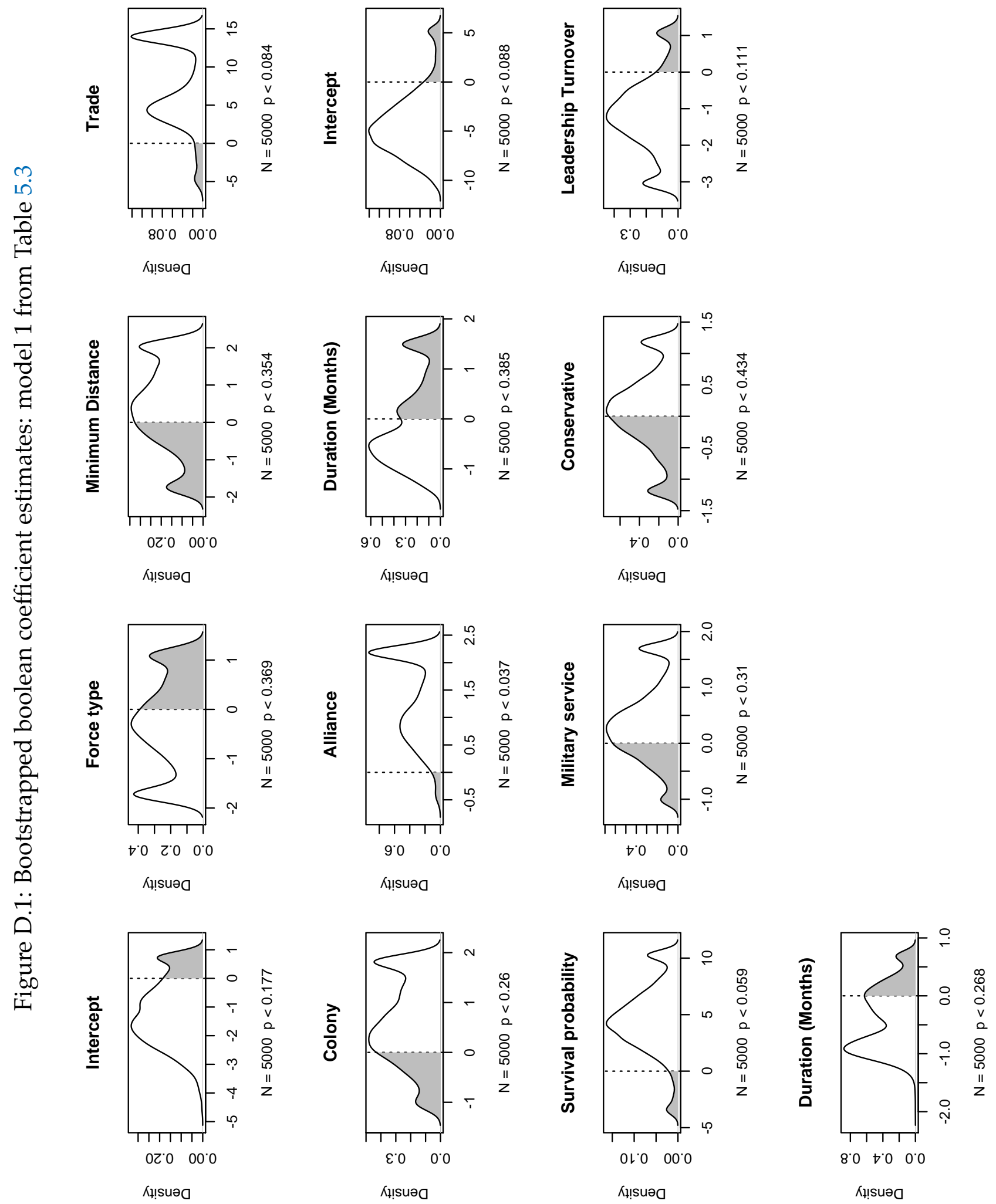




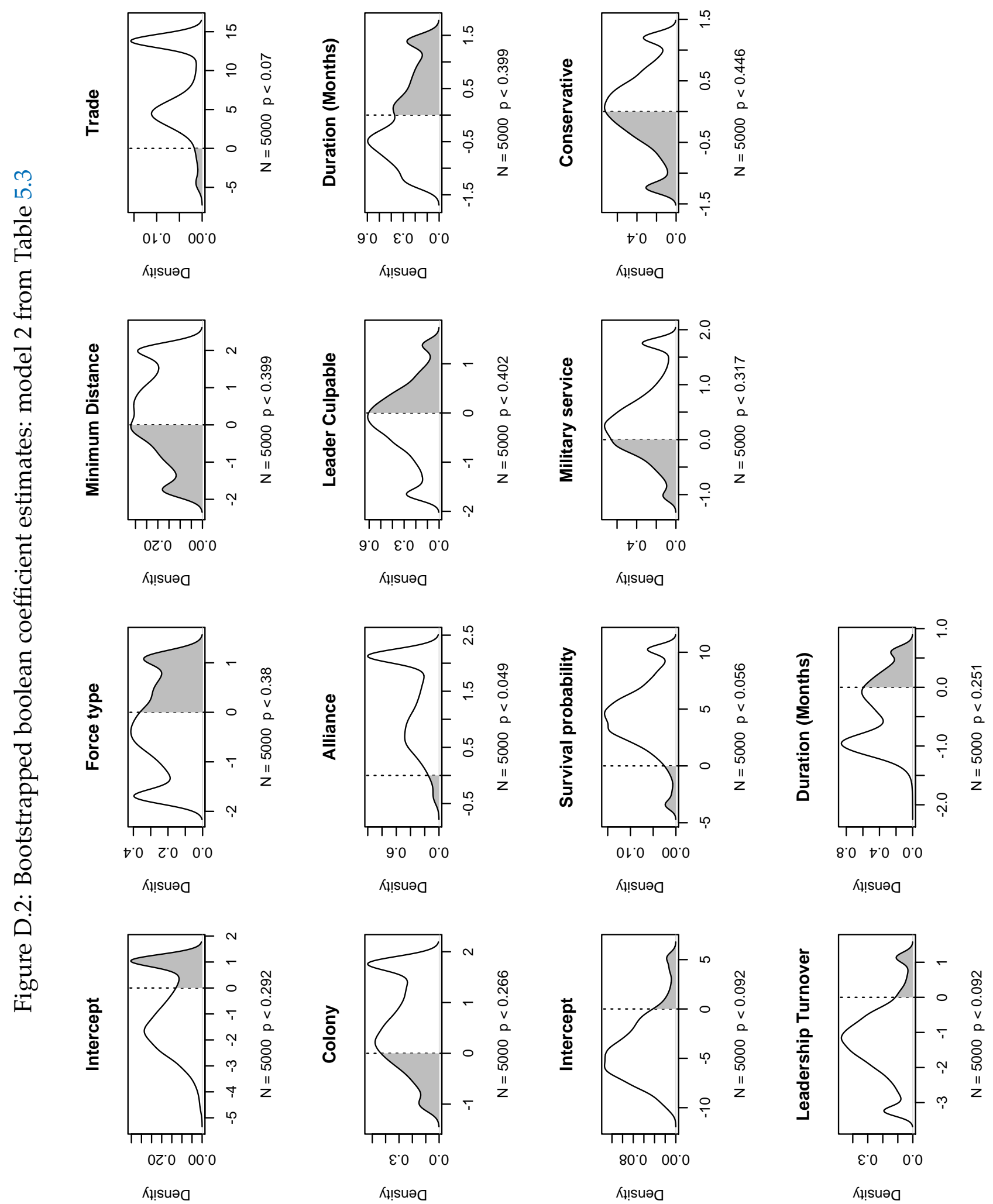




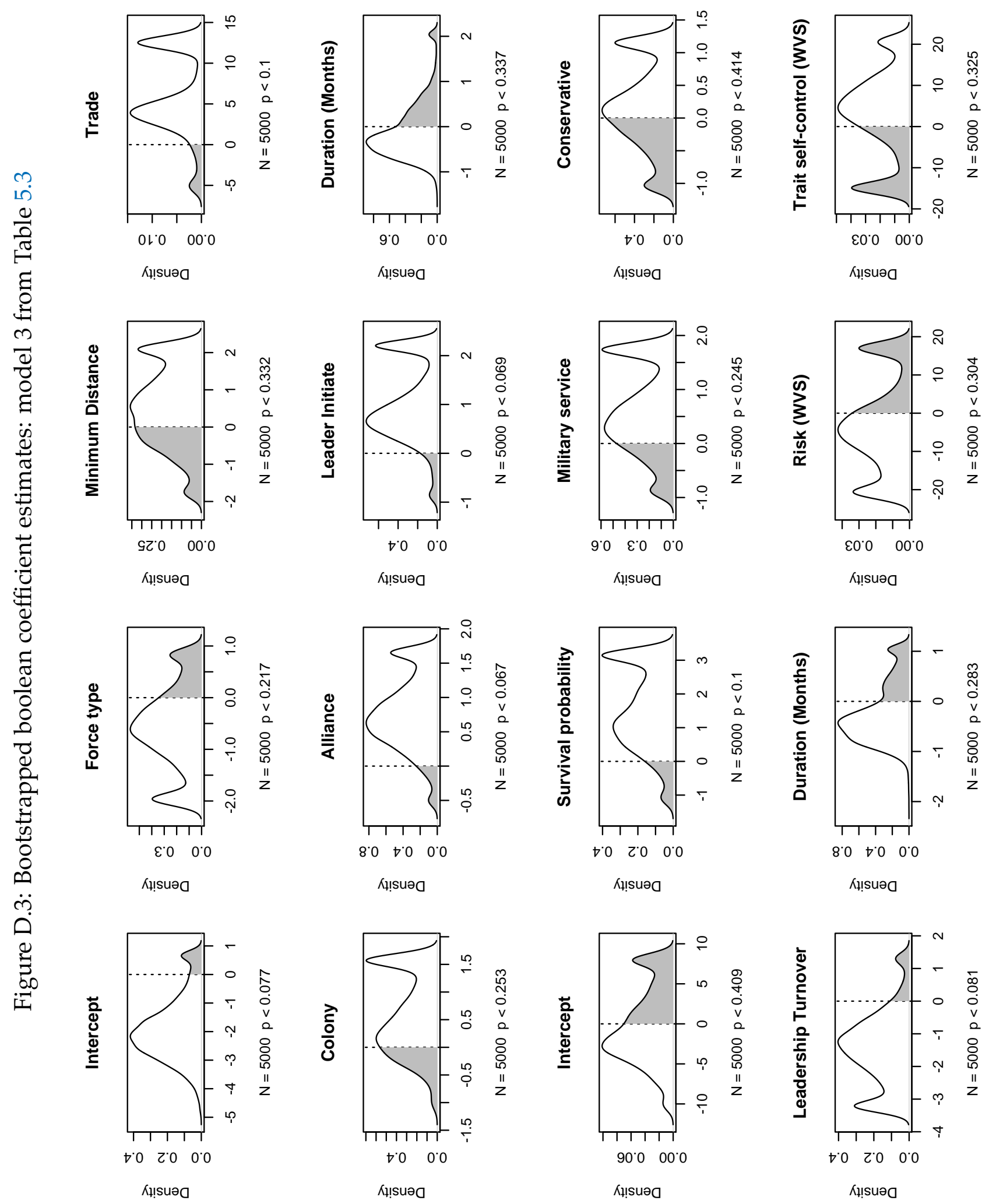




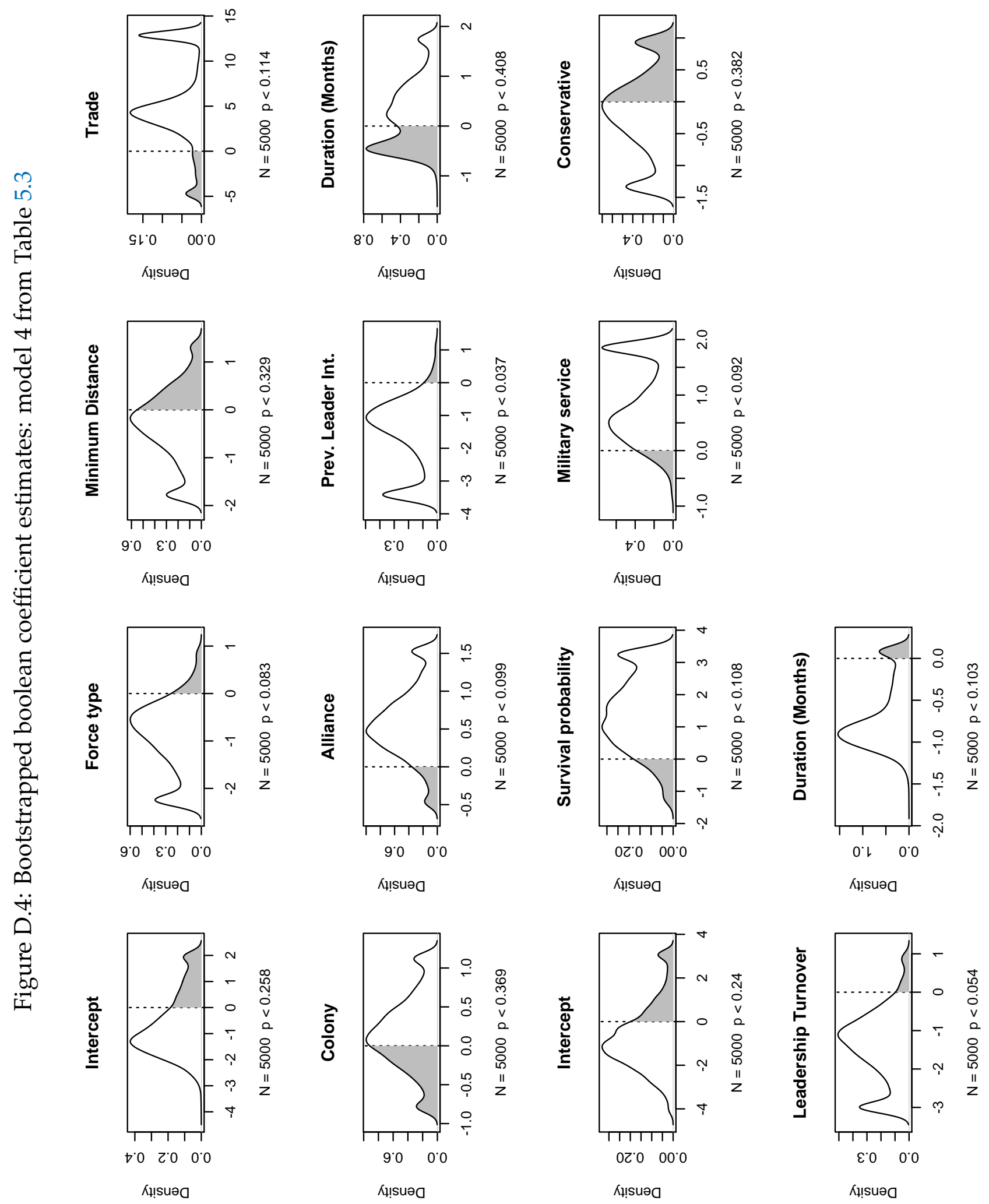




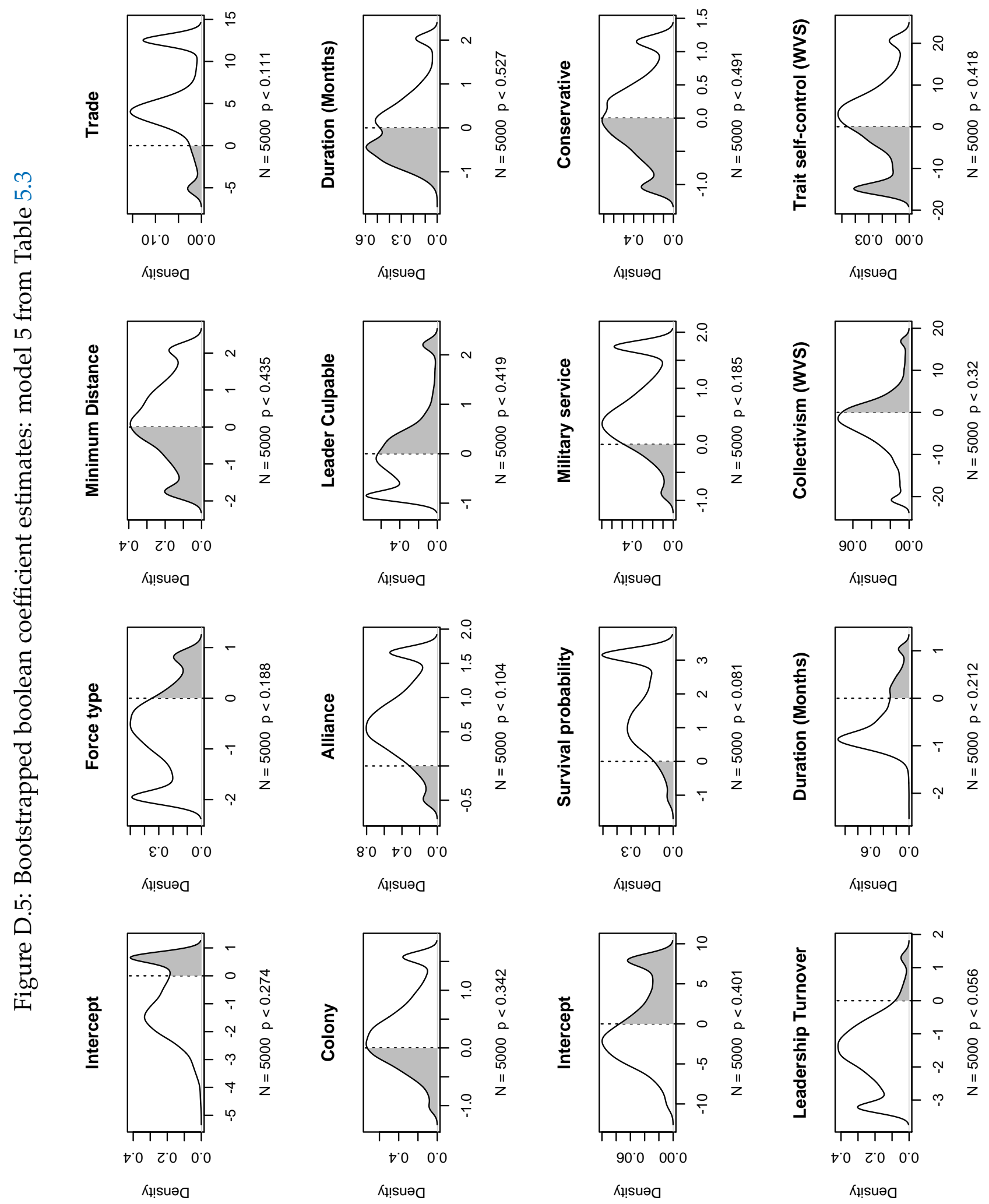




\section{Bibliography}

Achen, Christopher H. 1977. "Measuring Representation: Perils of the Correlation Coefficient." American Journal of Political Science 21 (4): 805-815.

Achen, Christopher H. 2002. "Toward a New Political Methodology: Microfoundations and ART." Annual Review of Political Science 5: 423-450.

Ackerman, Joshua M., Noah J. Goldstein, Jenessa R. Shapiro, and John A. Bargh. 2009. "You Wear Me Out: The Vicarious Depletion of Self-Control." Psychological Science 20 (3): 326-332.

Agnew, Christopher R., Aaron M. Hoffman, Justin J. Lehmiller, and Natasha T. Duncan. 2007. "From the Interpersonal to the International: Understanding Commitment to the "War on Terror"." Personality and Social Psychology Bulletin 33 (11): 1559-1571.

Ainslie, George. 1986. "Beyond microeconomics: Conflict among interests in a multiple self as a determinant of value." In The Multiple Self, ed. Jon Elster. Cambridge: Cambridge University Press.

Ainslie, George. 1992. Picoeconomics: The Strategic Interaction of Successive Motivational States within the Person. Cambridge: Cambridge University Press.

Ainslie, George. 2001. Breakdown of Will. Cambridge: Cambridge University Press.

Alford, John R., Carolyn L. Funk, and John R. Hibbing. 2005. “Are Political Orientations Genetically Transmitted?" American Political Science Review 99 (2): 153-167.

Allison, Graham. 1971. Essence of Decision. Boston: Little Brown and Company.

Almond, Gabriel A. 1950. The American People and Foreign Policy. New York: Harcourt, Brace and Company.

Alt, James E., Randall L. Calvert, and Brian Humes. 1988. "Reputation and Hegemonic Stability: A Game-Theoretic Analysis." American Political Science Review 82 (2): 445-66.

Althaus, Scott L. Forthcoming. Time in the Fight: Understanding Popular Support for America's Wars. New York: Cambridge University Press.

Althaus, Scott L., and Kevin Coe. 2011. "Social Identity Processes and the Dynamics of Public Support for War." Public Opinion Quarterly 75 (1): 65-88. 
Alvarez, R. Michael, and John Brehm. 1995. “American Ambivalence Towards Abortion Policy: Development of a Heteroskedastic Probit Model of Competing Values." American Journal of Political Science 39 (4): 1055-1082.

Ameriks, John, Andrew Caplin, John Leahy, and Tom Tyler. 2007. “Measuring SelfControl Problems." American Economic Review 97 (3): 966-972.

Anastasi, Anne. 1937. Differential Psychology - Individual and Group Differences in Behavior. New York: MacMillan.

Arendt, Hannah. 1971. The Life of the Mind. Vol. II: Willing Orlando, FL: Harcourt.

Arkes, Hal R., and Catherine Blumer. 1985. "The psychology of sunk cost." Organizational Behavior and Human Decision Processes 35 (1): 124-140.

Arneklev, Bruce J., Harold G. Grasmick, and Robert J. Bursik. 1999. "Evaluating the Dimensionality and Invariance of "Low Self-Control"." Journal of Quantitative Criminology 15 (3): 307-331.

Arreguín-Toft, Ivan. 2001. "How the Weak Win Wars: A Theory of Asymmetric Conflict." International Security 26 (1): 93-128.

Atran, Scott. 2010. "A Question of Honour: Why the Taliban Fight and What to Do About It." Asian Journal of Social Science 38 (3): 343-363.

Atran, Scott, Robert Axelrod, and Richard Davis. 2007. "Sacred Barriers to Conflict Resolution." Science 317: 1039-1040.

Axelrod, Robert. 1984. The Evolution of Cooperation. New York: Basic Books.

Axelrod, Robert. 1997. The Complexity of Cooperation: Agent-Based Models of Cooperation and Collaboration. Princeton, NJ: Princeton University Press.

Azam, Jean-Paul. 2005. “Suicide-bombing as inter-generational investment." Public Choice 122 (1-2): 177-198.

Bak, Daehee, and Glenn Palmer. 2010. "Testing the Biden Hypotheses: Leader Tenure, Age, and International Conflict." Foreign Policy Analysis 6 (3): 257-273.

Baldwin, David. 1979. "Power Analysis and World Politics: New Trends versus Old Tendencies." World Politics 31 (2): 161-194.

Barbieri, Katherine, Omar M.G. Keshk, and Brian Pollins. 2009. “Trading Data: Evaluating our Assumptions and Coding Rules." Conflict Management and Peace Science 26 (5): 471-491.

Barkin, J. Samuel. 2004. “Time Horizons and Multilateral Enforcement in International Cooperation." International Studies Quarterly 48 (2): 363-382. 
Baron, Reuben M., and David A. Kenny. 1986. "The moderator-mediator variable distinction in social psychological research: Conceptual, strategic, and statistical considerations." Journal of Personality and Social Psychology 51 (6): 1173-1182.

Bartone, Paul T. 2006. "Resilience Under Military Operational Stress: Can Leaders Influence Hardiness?" Military Psychology 18: 131-148.

Bartram, Dave. 2013. "Scalar Equivalence of OPQ32: Big Five Profiles of 31 Countries." Journal Of Cross-Cultural Psychology 44 (1): 61-83.

Bas, Muhammet A. 2012. "Measuring Uncertainty in International Relations: Heteroskedastic Strategic Models." Conflict Management and Peace Science 29 (5): 490520 .

Bassin, Mark. 1993. "Turner, Solov'ev, and the "Frontier Hypothesis"." Journal of Modern History 65 (3): 473-511.

Bauer, Daniel J. 2011. "Explaining Individual Differences in Psychological Processes." Current Directions in Psychological Science 20 (2): 115-118.

Baum, Matthew A. 2002. "The Constituent Foundations of the Rally-Round-theFlag Phenomenon." International Studies Quarterly 46 (2): 263-298.

Baum, Matthew A. 2004. "Going Private: Public Opinion, Presidential Rhetoric, and the Domestic Politics of Audience Costs in U.S. Foreign Policy Crises." Journal of Conflict Resolution 48 (5): 603-631.

Baumeister, Roy F., and Julie Juola Exline. 1999. "Virtue, Personality, and Social Relations: Self-Control as the Moral Muscle." Journal of Personality 67 (6): 11651194.

Baumeister, Roy F., and Kathleen D. Vohs. 2004. Handbook of Self-Regulation: Research, Theory, and Applications. New York: The Guilford Press.

Baumeister, Roy F., Kathleen D. Vohs, and Dianne M. Tice. 2007. “The Strength Model of Self-Control." Current Directions in Psychological Science 16 (6): 351-355.

Baynes, John. 1967. Morale: A Study of Men and Courage. Frederick A. Praeger.

Beardsley, Kyle. 2008. "Agreement without Peace? International Mediation and Time Inconsistency Problems." American Journal of Political Science 52 (4): 723-740.

Beaver, Kevin M., Matt DeLisi, Michael G. Vaughn, and John Paul Wright. 2010. "The Intersection of Genes and Neuropsychological Deficits in the Prediction of Adolescent Delinquency and Low Self-Control." International Journal of Offender Therapy and Comparative Criminology 54 (1): 22-42.

Becker, Howard S. 1961. "Notes on the Concept of Commitment." American Journal of Sociology 66 (1): 32-40. 
Bem, Daryl J. 1967. "Self-Perception: An Alternative Interpretation of Cognitive Dissonance Phenomena." Psychological Review 74 (3): 183-200.

Bénabou, Roland, and Jean Tirole. 2002. "Self-Confidence and Personal Motivation." The Quarterly Journal of Economics 117 (3): 871-915.

Bénabou, Roland, and Jean Tirole. 2003. "Intrinsic and Extrinsic Motivation." Review of Economic Studies 70 (3): 489-520.

Bénabou, Roland, and Jean Tirole. 2004. "Willpower and Personal Rules." Journal of Political Economy 112 (4): 848-886.

Benhabib, Jess, and Alberto Bisin. 2005. "Modelling Internal Commitment Mechanisms and Self-Control: A Neuroeconomics Approach to Consumption-Saving Decisions." Games and Economic Behavior 52 (2): 460-492.

Benjamin, Daniel J., James J. Choi, and A. Joshua Strickland. 2010. "Social Identity and Preferences." American Economic Review 100 (4): 1913-1928.

Bennett, D. Scott. 1999. "Parametric Models, Duration Dependence, and TimeVarying Data Revisited." American Journal of Political Science 43 (1): 256-270.

Bennett, D. Scott, and Allan C Stam. 1998. "The Declining Advantages of Democracy: A Combined Model of War Outcomes and Duration." Journal of Conflict Resolution 42 (3): 344-366.

Bennett, D. Scott, and Allan C Stam. 2000. "EUGene: A conceptual manual." International Interactions 26: 179-204.

Berejekian, Jeffrey. 1997. “The Gains Debate: Framing State Choice.” American Political Science Review 91 (4): 789-805.

Berinsky, Adam J. 2007. "Assuming the Costs of War: Events, Elites, and American Public Support for Military Conflict.” Journal of Politics 69 (4): 975-97.

Besley, Timothy, and Marta Reynal-Querol. 2011. “Do Democracies Select More Educated Leaders?" American Political Science Review 105 (3): 552-566.

Betts, Richard K. 1980. “Comment on Mueller: Interests, Burdens, and Persistence: Asymmetries Between Washington and Hanoi." International Studies Quarterly 24 (4): 520-524.

Bially Mattern, Janice. 2005. “Why Soft Power Isn't So Soft: Representational Force and the Sociolinguistic Construction of Attraction in World Politics." Millennium: Journal of International Studies 33 (3): 583-612.

Bieler, Andreas, and Adam David Morton. 2001. "The Gordian Knot of AgencyStructure in International Relations: A Neo-Gramscian Perspective." European Journal of International Relations 7 (1): 5-35. 
Bilmes, Linda J., and Joseph E. Stiglitz. 2012. "Estimating the Costs of War: Methodological Issues, with Applications to Iraq and Afghanistan." In Oxford Handbook of the Economics of Peace and Conflict. Oxford University Press.

Blainey, Geoffrey. 1973. The Causes of War. New York: Free Press.

Boettcher, William A. III. 1995. “Context, Methods, Numbers, And Words: Prospect Theory in International Relations." Journal of Conflict Resolution 39 (3): 561-583.

Boettcher, William A. III, and Michael D. Cobb. 2006. "Echoes of Vietnam? Casualty Framing and Public Perceptions of Success and Failure in Iraq." Journal of Conflict Resolution 50 (6): 831-854.

Boettcher, William A. III, and Michael D. Cobb. 2009. "'Don't Let Them Die in Vain": Casualty Frames and Public Tolerance for Escalating Commitment in Iraq." Journal of Conflict Resolution 53 (5): 677-697.

Bollen, Kenneth, A. 1989. Structural equations with latent variables. New York: John Wiley \& Sons.

Bouchard, Thomas J., Jr. 1994. "Genes, Environment, and Personality." Science 264 (5166): 1700-1701.

Boutwell, Brian B., and Kevin M. Beaver. 2010. “The Intergenerational Transmission of Low Self-control." Journal of Research in Crime and Delinquency 47 (2): 174-209.

Box-Steffensmeier, Janet M., and Bradford S. Jones. 2004. Event History Modeling: A Guide for Social Scientists. New York: Cambridge University Press.

Box-Steffensmeier, Janet M., and Christopher Zorn. 2001. "Duration Models and Proportional Hazards in Political Science." American Journal of Political Science 45 (4): 972-988.

Braumoeller, Bear F. 2003. "Causal Complexity and the Study of Politics." Political Analysis 11 (3): 209-233.

Braumoeller, Bear F., and Austin Carson. 2011. "Political Irrelevance, Democracy, and the Limits of Militarized Conflict." Journal of Conflict Resolution 55 (2): 292320.

Brehm, John. 1993. The Phantom Respondents. Opinion Surveys and Political Representation.

Brennan, Timothy J. 1989. "A Methodological Assessment of Multiple Utility Frameworks." Economics and Philosophy 5 (2): 189-208.

Bueno de Mesquita, Bruce, and Randolph M. Siverson. 1995. "War and the Survival of Political Leaders: A Comparative Study of Regime Types and Political Accountability." American Political Science Review 89 (4): 841-855. 
Byman, Daniel L., and Kenneth M. Pollack. 2001. "Let Us Now Praise Great Men: Bringing the Statesman Back In." International Security 25 (4): 107-146.

Carlsnaes, Walter. 1992. “The Agent-Structure Problem in Foreign Policy Analysis.” International Studies Quarterly 36 (3): 245-270.

Carter, David B., and Curtis S. Signorino. 2010. “Back to the Future: Modeling Time Dependence in Binary Data." Political Analysis 18 (3): 271-292.

Caspi, Avshalom. 2000. "The Child Is Father of the Man: Personality Continuities from Childhood to Adulthood." Journal of Personality and Social Psychology 78 (1): 158-172.

Caspi, Avshalom, and Terrie E. Moffitt. 2006. “Gene-environment interactions in psychiatry: joining forces with neuroscience." Nature Reviews Neuroscience 7: 583590.

Castles, Francis G., and Peter Mair. 1984. “Left-Right Political Scales: Some 'Expert' Judgments." European Journal of Political Research 12 (1): 73-88.

Caverley, Jonathan D. 2009/10. “The Myth of Military Myopia: Democracy, Small Wars, and Vietnam." International Security 34 (3): 119-157.

Cederman, Lars-Erik. 1997. Emergent Actors in World Politics: How States \& Nations Develop $\mathcal{E}$ Dissolve. Princeton, NJ: Princeton University Press.

Cederman, Lars-Erik. 2003. "Modeling the Size of Wars: From Billiard Balls to Sandpiles." American Political Science Review 97 (1): 135-150.

Chait, Jonathan. 2011. "Paul Ryan's Norquistian-Churchillian Foreign Policy.” In The New Republic. http://www . newrepublic.com/blog/jonathan-chait/89382/ paul-ryan-foreign-policy-american-exceptionalism-obama.

Chambers, John R., Nicholas Epley, Kenneth Savitsky, and Paul D. Windschitl. 2008. "Knowing Too Much: Using Private Knowledge to Predict How One Is Viewed by Others." Psychological Science 19 (6): 542-548.

Chandler, David. 2013. "International Statebuilding and the Ideology of Resilience." Politics Forthcoming.

Chandler, David G. 1966. The Campaigns of Napoleon. MacMillan.

Charlesworth, James C. 1967. "National Character in the Perspective of Political Science." Annals of the American Academy of Political and Social Science 370: 23-29.

Charney, Evan, and William English. 2012. "Candidate Genes and Political Behavior." American Political Science Review 106 (1): 1-34. 
Cheng, Ken, Jennifer Pena, Melanie A. Porter, and Julia D. Irwin. 2002. "Self-control in honeybees." Psychonomic Bulletin E Review 9 (2): 259-263.

Chiozza, Giacomo, and H.E. Goemans. 2004. "International Conflict and the Tenure of Leaders: Is War Still Ex Post Inefficient?" American Journal of Political Science 48 (3): 604-619.

Chiozza, Giacomo, and H.E. Goemans. 2011. Leaders and International Conflict. Cambridge, UK: Cambridge University Press.

Clark, David H. 2003. “Can Strategic Interaction Divert Diversionary Behavior? A Model of U.S. Conflict Propensity." Journal of Politics 65 (4): 1013-1039.

Clark, David H., and Patrick M. Regan. 2003. “Opportunities to Fight: A Statistical Technique For Modeling Unobservable Phenomena." Journal of Conflict Resolution 47 (1): 94-115.

Clauset, Aaron, Maxwell Young, and Kristian Skrede Gleditsch. 2007. "On the Frequency of Severe Terrorist Events." Journal of Conflict Resolution 51 (1): 58-87.

Cline, Ray S. 1975. World Power Assessment: A Calculus of Strategic Drift. Boulder, Colorado: Westview Press.

Cline, Ray S. 1994. The Power of Nations in the 1990s. Lanham, MD: University Press of America.

Condra, Luke N., and Jacob N. Shapiro. 2012. "Who Takes the Blame? The Strategic Effects of Collateral Damage." American Journal of Political Science 56 (1): 167-187.

Converse, Philip E. 1964. "The nature and origin of belief systems in mass publics." In Ideology and Discontent, ed. David E. Apter. New York: Free Press.

Copeland, Dale C. 2000. The Origins of Major War. Ithaca: Cornell University Press.

Corno, Lyn. 1993. “The Best-Laid Plans: Modern Conceptions of Volition and Educational Research." Educational Researcher 22 (2): 14-22.

Croco, Sarah E. 2011. “The Decider's Dilemma: Leader Culpability, War Outcomes, and Domestic Punishment." American Political Science Review 105 (August 2011): 457-477.

Dafoe, Allan, and Devin M. Caughey. N.d. "Honor and War: Using Southern Presidents to Identify Reputational Effects in International Conflict." Unpublished manuscript.

Dahl, Robert A. 1957. “The concept of power.” Systems Research 2 (3): 201-215.

Davenport, John. 2007. Will as Commitment and Resolve: An Existential Account of Creativity, Love, Virtue, and Happiness. New York: Fordham University Press. 
de Ridder, Denise T.D., Gerty Lensvelt-Mulders, Catrin Finkenauer, F. Marijn Stok, and Roy F. Baumeister. 2012. "Taking Stock of Self-Control: A Meta-Analysis of How Trait Self-Control Relates to a Wide Range of Behaviors." Personality and Social Psychology Review 16 (1): 76-99.

Dehghani, Morteza, Rumen Iliev, Sonya Sachdeva, Scott Atran, Jeremy Ginges, and Douglas Medin. 2009. “Emerging sacred values: Iran's nuclear program.” Judgment and Decision Making 4 (7): 930-933.

Dehghani, Morteza, Scott Atran, Rumen Iliev, Sonya Sachdeva, Douglas Medin, and Jeremy Ginges. 2010. "Sacred values and conflict over Iran's nuclear program." Judgment and Decision Making 5 (7): 540-546.

Dennett, Daniel C. 1987. The Intentional Stance. Cambridge, MA: MIT Press.

Desch, Michael C. 1998. "Culture Clash: Assessing the Importance of Ideas in Security Studies." International Security 23 (1): 141-170.

Dessler, David. 1989. "What's At Stake in the Agent-Structure Debate?" International Organization 43 (3): 441-73.

Diamantopoulos, Adamantios, and Heidi M. Winklhofer. 2001. "Index Construction with Formative Indicators: An Alternative to Scale Development." Journal of Marketing Research 38 (2): 269-277.

Dickson, Eric S. 2009. “Do Participants and Observers Assess Intentions Differently During Bargaining and Conflict?" American Journal of Political Science 53 (4): 910930.

Dolar, Mladen. 1992. "Hitchcock's Objects." In Everything you always wanted to know about Lacan (but were afraid to ask), ed. Slavoj Žižek. Verso.

Dorfleitner, Gregor, and Michael Krapp. 2007. "On multiattributive risk aversion: some clarifying results." Review of Managerial Science 1 (1): 47-63.

Döring, Holger, and Philip Manow. 2012. "Parliament and government composition database (ParlGov): An Infrastructure for empirical information on parties, elections and governments in modern democracies." www. parlgov. org.

Doty, Roxanne Lynn. 1997. “Aporia: A Critical Exploration of the Agent-Structure Problematique in International Relations Theory." European Journal of International Relations 3 (3): 365-392.

Downs, George W., and David M. Rocke. 1995. Optimal Imperfection? Domestic Uncertainty and Institutions in International Relations. Princeton, NJ: Princeton University Press. 
Dreher, Axel, Michael J. Lamla, Sarah M. Lein, and Frank Somogyi. 2009. “The impact of political leaders' profession and education on reforms." Journal of Comparative Economics 37 (1): 169 - 193.

Druckman, James N., and Cindy D. Kam. 2011. "Students as Experimental Participants: A Defense of the "Narrow Data Base"." In Cambridge Handbook of Political Science, ed. James N. Druckman, Donald P. Green, James H. Kuklinski, and Arthur Lupia. Cambridge University Press.

Druckman, James N., and Thomas J. Leeper. 2012. “Learning More from Political Communication Experiments: Pretreatment and Its Effects." American Journal of Political Science 56 (4): 875-896.

Duckworth, Angela Lee, and Margaret L. Kern. 2011. "A Meta-Analysis of the Convergent Validity of Self-Control Measures." Journal of Research in Personality 45 (3): 259-268.

Dunn, Robert. 1987. The Possibility of Weakness of Will. Indianapolis: Hackett.

Dunning, David, Chip Heath, and Jerry M. Suls. 2004. “Flawed Self-Assessment: Implications for Health, Education, and the Workplace." Psychological Science in the Public Interest 5: 69-106.

Dyson, Stephen Benedict. 2007. "Alliances, Domestic Politics, and Leader Psychology: Why Did Britain Stay Out of Vietnam and Go into Iraq?" Political Psychology 28 (6): 647-666.

Eckles, David L., and Brian F. Schaffner. 2011. "Risk Tolerance and Support for Potential Military Interventions." Public Opinion Quarterly 75 (3): 533-544.

Edwards, Jeffrey R., and Richard P. Bagozzi. 2000. "On the Nature and Direction of Relationships Between Constructs and Measures." Psychological Methods 5 (2): 155-174.

Ehrlich, Sean, and Cherie Maestas. 2010. "Risk Orientation, Risk Exposure, and Policy Opinions: The Case of Free Trade." Political Psychology 31 (5): 657-684.

Elias, Norbert. 2000. The Civilizing Process. Revised ed. Oxford: Blackwell Publishers.

Elster, Jon. 1979. Ulysses and the Sirens. Cambridge: Cambridge University Press.

Elster, Jon. 1982. "The Case for Methodological Individualism." Theory and Society 11 (4): 453-482.

Elster, Jon. 1985. Making Sense of Marx. Cambridge, UK: Cambridge University Press.

Elster, Jon. 1986. The Multiple Self. Cambridge: Cambridge University Press. 
Elster, Jon. 1989. Nuts and Bolts for the Social Sciences. Cambridge, UK: Cambridge University Press.

Elster, Jon. 2000. Ulysses Unbound: Studies in Rationality, Precommitment, and Constraints. Cambridge: Cambridge University Press.

Elster, Jon. 2006. "Weakness of will and preference reversals." In Understanding Choice, Explaining Behavior: Essays in Honour of Ole-Jørgen Skog, ed. Jon Elster, Gjelsvik, Aanund Hylland, and Karl Moene. Oslo: Unipub Forlag.

Endler, Norman S., and David Magnusson. 1976. "Toward an Interactional Psychology of Personality." Psychological Bulletin 83 (5): 956-974.

Epley, Nicholas, and David Dunning. 2000. "Feeling "Holier Than Thou": Are SelfServing Assessments Produced by Errors in Self- or Social Prediction?" Journal of Personality and Social Psychology 79 (6): 861-875.

Epley, Nicholas, and Eugene M. Caruso. 2008. "Perspective Taking: Misstepping Into Others' Shoes." In The handbook of imagination and mental simulation, ed. Keith D. Markman, William M.P. Klein, and Julie A. Suhr. New York: Psychology Press.

Etzioni, Amitai. 1986. “The Case for a Multiple-Utility Conception.” Economics and Philosophy 2 (2): 159-183.

Eyal, Tal, Nira Liberman, Yaacov Trope, and Eva Walther. 2004. “The Pros and Cons of Temporally Near and Distant Action." Journal of Personality and Social Psychology 86 (6): 781-795.

Fearon, James. 1995. “Rationalist Explanations for War." International Organization 49 (3): 379-414.

Fearon, James. 1997. “Tying Hands versus Sinking Costs: Signaling Foreign Policy Interests." Journal of Conflict Resolution 41 (1): 68-90.

Fearon, James D. 1994. "Domestic Political Audiences and the Escalation of International Disputes." American Political Science Review 88 (3): 577-592.

Fearon, James D. 1998. “Domestic Politics, Foreign Policy, and Theories of International Relations." Annual Review of Political Science 1: 289-313.

Fearon, James D. 2002. "Selection Effects and Deterrence." International Interactions 28 (1): 5-29.

Feaver, Peter D., and Christopher Gelpi. 2005. Choosing Your Battles: American CivilMilitary Relations and the Use of Force. Princeton: Princeton University Press.

Feinerer, Ingo, Kurt Hornik, and David Meyer. 2008. “Text Mining Infrastructure in R." Journal of Statistical Software 25 (5). 
Feldman, Ofer and Linda O. Valenty, eds. 2001. Profiling political leaders: crosscultural studies of personality and behavior. Westport, CT: Praeger.

Fennell, Jonathan. 2011. Combat and Morale in the North African Cmpaign: The Eighth Army and the Path to el Alamein. Cambridge University Press.

Fennell, Lee Anne. 2009. "Willpower and Legal Policy." Annual Review of Law and Social Science 5: 91-113.

Fettweis, Christopher J. 2007. "Credibility and the War on Terror." Political Science Quarterly 122 (4): 607-633.

Fey, Mark, and Kristopher W. Ramsay. 2006. “The Common Priors Assumption: A Comment on "Bargaining and the Nature of War"." Journal of Conflict Resolution 50 (4): 607-613.

Fey, Mark, and Kristopher W. Ramsay. 2007. "Mutual Optimism and War." American Journal of Political Science 51 (4): 738-754.

Fey, Mark, and Kristopher W. Ramsay. 2011. "Uncertainty and Incentives in Crisis Bargaining: Game-Free Analysis of International Conflict." American Journal of Political Science 55 (1): 149-169.

Field, Christopher, and Alan H. Welsh. 2007. "Bootstrapping clustered data." Journal of the Royal Statistical Society: Series B 69: 369-390.

Filson, Darren, and Suzanne Werner. 2002. "A Bargaining Model of War and Peace: Anticipating the Onset, Duration, and Outcome of War." American Journal of Political Science 46 (4): 819-838.

Filson, Darren, and Suzanne Werner. 2004. "Bargaining and Fighting: The Impact of Regime Type on War Onset, Duration, and Outcomes." American Journal of Political Science 48 (2): 296-313.

Filson, Darren, and Suzanne Werner. 2007a. "The Dynamics of Bargaining and War." International Interactions 33 (1): 31-50.

Filson, Darren, and Suzanne Werner. 2007b. "Sensitivity to Costs of Fighting versus Sensitivity to Losing the Conflict." Journal of Conflict Resolution 51 (5): 691-714.

Finkel, Eli J., Erica B. Slotter, C. Nathan DeWall, Megan Oaten, and Vangie A. Foshee. 2009. "Self-Regulatory Failure and Intimate Partner Violence Perpetration." Journal of Personality and Social Psychology 97 (3): 483-499.

Finkenauer, Catrin, Rutger C.M.E. Engels, and Roy F. Baumeister. 2005. “Parenting behaviour and adolescent behavioural and emotional problems: The role of selfcontrol." International Journal of Behavioral Development 29 (1): 58-69. 
Fischer, David Hackett. 1989. Albion's Seed: Four British Folkways in America. New York: Oxford University Press.

Fleeson, William. 2001. "Toward a Structure- and Process-Integrated View of Personality: Traits as Density Distributions of States." Journal of Personality and Social Psychology 80 (6): 1011-1027.

Fleeson, William. 2004. "Moving Personality Beyond the Person-Situation Debate." Current Directions in Psychological Science 13 (2): 83-87.

Fletcher, Joseph F., Heather Bastedo, and Jennifer Hove. 2009. "Losing Heart: Declining Support and the Political Marketing of the Afghanistan Mission." Canadian Journal of Political Science 42 (4): 911-937.

Flores, Thomas Edward, and Irfan Nooruddin. 2009. "Financing the Peace: Evaluating World Bank Post-Conflict Assistance Programs." Review of International Organizations 4 (1): 1-27.

Fowler, James H., and Christopher T. Dawes. 2008. "Two Genes Predict Voter Turnout." Journal of Politics 70 (3): 579-594.

Frankfurt, Harry G. 1971. "Freedom of the Will and the Concept of a Person." The Journal of Philosophy 68 (1): 5-20.

Frederick, Shane. 2006. "Valuing future life and future lives: A framework for understanding discounting." Journal of Economic Psychology 27 (5): 667-680.

Freese, Jeremy. 2010. "Preferences." In Oxford Handbook of Analytical Sociology, ed. Peter Hedstrom and Peter Bearman. Oxford: Oxford University Press.

Friedman, Milton. 1953. “The Methodology of Positive Economics." In Essays in Positive Economics. Chicago: University of Chicago Press.

Fritsche, Immo, Eva Jonas, and Thomas Fankhänel. 2008. “The Role of Control Motivation in Mortality Salience Effects on Ingroup Support and Defense." Journal of Personality and Social Psychology 95 (3): 524-541.

Fudenberg, Drew, and David K. Levine. 2006. “A Dual-Self Model of Impulse Control." American Economic Review 96 (5): 1449-1476.

Fujita, Kentaro, and Joseph C. Roberts. 2010. "Promoting prospective self-control through abstraction." Journal of Experimental Social Psychology 46 (6): 1049-1054.

Furlong, Kathryn, Nils Petter Gleditsch, and Håvard Hegre. 2006. “Geographic Opportunity and Neomalthusian Willingness: Boundaries, Shared Rivers, and Conflict." International Interactions 32 (1): 79-108.

Gaines, Brian J., James H. Kuklinski, and Paul J. Quirk. 2007. "The Logic of the Survey Experiment Reexamined." Political Analysis 15 (1): 1-20. 
Galliot, Matthew T., and Roy F. Baumeister. 2007. "The Physiology of Willpower: Linking Blood Glucose to Self-Control." Personality and Social Psychology Review 11 (4): 303-327.

Galliot, Matthew T., Roy F. Baumeister, C. Nathan DeWall, Jon K. Maner, Ashby Plant, Dianne M. Tice, Lauren E. Brewer, and Brandon J. Schmeichel. 2007. "SelfControl Relies on Glucose as a Limited Energy Source: Willpower Is More Than a Metaphor." Journal of Personality and Social Psychology 92 (2): 325-336.

Gartner, Scott Sigmund. 1997. Strategic Assessment in War. New Haven, CT: Yale University Press.

Gartner, Scott Sigmund. 2008a. "The Multiple Effects of Casualties on Public Support for War: An Experimental Approach." American Political Science Review 102 (1): 95-106.

Gartner, Scott Sigmund. 2008b. "Secondary Casualty Information: Casualty Uncertainty, Female Casualties, and Wartime Support." Conflict Management and Peace Science 25 (2): 98-111.

Gartner, Scott Sigmund, and Gary M. Segura. 1998. "War, Casualties, and Public Opinion." Journal of Conflict Resolution 42 (3): 278-300.

Gartner, Scott Sigmund, Gary M. Segura, and Michael Wilkening. 1997. "All Politics Are Local: Local Losses and Individual Attitudes toward the Vietnam War." Journal of Conflict Resolution 41 (5): 669-694.

Gartzke, Eric. 1998. "Kant We All Just Get Along? Opportunity, Willingness, and the Origins of the Democratic Peace." American Journal of Political Science 42 (1): $1-27$.

Gartzke, Eric. 2001. "Democracy and the Preparation for War: Does Regime Type Affect States' Anticipation of Casualties?" International Studies Quarterly 45 (3): 467-484.

Gartzke, Eric, Quan Li, and Charles Boehmer. 2001. "Investing in the Peace: Economic Interdependence and International Conflict." International Organization 55 (2): 391-438.

Gelman, Andrew. 2007. "Struggles with Survey Weighting and Regression Modeling." Statistical Science 22 (2): 153-164.

Gelpi, Christopher F., and Michael Griesdorf. 2001. "Winners or Losers? Democracies in International Crisis, 1918-94." American Political Science Review 95 (3): 633-647.

Gelpi, Christopher, Jason Reifler, and Peter Feaver. 2007. “Iraq the Vote: Retrospective and Prospective Foreign Policy Judgments on Candidate Choice and Casualty Tolerance." Political Behavior 29 (2): 151-174. 
Gelpi, Christopher, and Joseph M. Grieco. 2001. "Attracting Trouble: Democracy, Leadership Tenure, and the Targeting of Militarized Challenges, 1918-1992." Journal of Conflict Resolution 45 (6): 794-817.

Gelpi, Christopher, Peter D. Feaver, and Jason Reifler. 2005/ 06. "Success Matters: Casualty Sensitivity and the War in Iraq." International Security 30 (3): 7-46.

Gelpi, Christopher, Peter D. Feaver, and Jason Reifler. 2009. Paying the Human Costs of War. Princeton: Princeton University Press.

George, Alexander L. 1969. "The "operational code": A neglected approach to the study of political leaders and decision making." International Studies Quarterly 13 (2): 190-222.

George, Alexander L., and William E. Simons. 1994. The Limits of Coercive Diplomacy. 2nd ed. Boulder, CO: Westview Press.

Gerring, John. 2007. "The Mechanistic Worldview: Thinking Inside the Box." British Journal of Political Science 38 (1): 161-179.

Geys, Benny. 2010. "Wars, Presidents and Popularity: The Political Cost(s) of War Re-examined." Public Opinion Quarterly 74 (2): 357-374.

Gibbs, John J., Dennis Giever, and Jamie S. Martin. 1998. “Parental Management and Self-Control: An Empirical Test of Gottfredson and Hirschi's General Theory." Journal of Research in Crime and Delinquency 35 (1): 40-70.

Gibler, Douglas M. 2007. “Bordering on Peace: Democracy, Territorial Issues, and Conflict." International Studies Quarterly 51 (3): 509-532.

Gibler, Douglas M. 2010. “Outside-In: The Effects of External Threat on State Centralization." Journal of Conflict Resolution 54 (4): 519-542.

Gifford, Adam, Jr. 2002. “Emotion and self-control." Journal of Economic Behavior \& Organization 49 (1): 113-130.

Gilbert, Daniel T. 1998. “Ordinary Personology." In The Handbook of Social Psychology, ed. Daniel T. Gilbert, Susan T. Fiske, and Gardner Lindzey. McGraw-Hill.

Ginges, Jeremy, and Scott Atran. 2011. "War as a moral imperative (not just practical politics by other means)." Proceedings of the Royal Society B: Biological Sciences 278 (February 16, 2011): 1-9.

Goddard, Stacie E. 2006. “Uncommon Ground: Indivisible Territory and the Politics of Legitimacy." International Organization 60 (1): 35-68.

Goemans, H.E., Kristian Skrede Gleditsch, and Giacomo Chiozza. 2009. "Introducing Archigos: A Data Set of Political Leaders." Journal of Peace Research 46 (2): 269-283. 
Goemans, Hein. 2000. War and Punishment: The Causes of War Termination and the First World War. Princeton, NJ: Princeton University Press.

Gottfredson, Michael, and Travis Hirschi. 1990. A General Theory of Crime. Stanford: Stanford University Press.

Grasmick, Harold G., Charles R. Tittle, Robert J. Bursik, and Bruce J. Arneklev. 1993. "Testing the Core Empirical Implications of Gottfredson and Hirschi's General Theory of Crime." Journal of Research in Crime and Delinquency 30 (1): 5-29.

Gray, Colin S. 1981. “National Style in Strategy: the American Example.” International Security 6 (2): 21-47.

Green, Donald P., and Holger L. Kern. 2012. "Modeling Heterogeneous Treatment Effects in Survey Experiments with Bayesian Additive Regression Trees." Public Opinion Quarterly 76 (3): 491-511.

Green, Donald P., Soo Yeon Kim, and David H. Yoon. 2001. "Dirty Pool." International Organization 55 (2): 441-468.

Green, Leonard, and Jr. Fisher, Edwin B. 1988. "Self-control in context." Behavioral and Brain Sciences 11 (4): 684-685.

Greenhill, Brian, Michael D. Ward, and Audrey Sacks. 2011. “The Separation Plot: A New Visual Method for Evaluating the Fit of Binary Models." American Journal of Political Science 55 (4): 990-1002.

Grieco, Joseph. 1993. "Anarchy and the Limits of Cooperation: A Realist Critique of the Newest Liberal Institutionalism." In Neorealism and Neoliberalism: The Contemporary Debate, ed. David A. Baldwin. New York: Columbia University Press.

Grimmer, Justin, and Gary King. 2011. “General purpose computer-assisted clustering and conceptualization." Proceedings of the National Academy of Sciences 108 (7): 2643-2650.

Gul, Faruk, and Wolfgang Pesendorfer. 2001. "Temptation and Self-Control." Econometrica 69 (6): 1403-1435.

Hafner-Burton, Emilie M., Brad L. LeVeck, David G. Victor, and James H. Fowler. 2013. “Decision Makers' Preferences for International Legal Cooperation." ILAR Working Paper \#13.

Hagger, Martin S., Chantelle Wood, Chris Stiff, and Nikos L.D. Chatzisarantis. 2010. "Ego Depletion and the Strength Model of Self-Control: A Meta-Analysis." Psychological Bulletin 136 (4): 495-525.

Haglund, David G. 2004. "What Good Is Strategic Culture? A Modest Defence of an Immodest Concept." International Journal 59 (3): 479-502. 
Haglund, David G., and Joshua D. Kertzer. 2008. “From Geo to Neo: A Speculative Inquiry into the Unusual "Geo-Ethnic" Roots of Neoconservatism in U.S. Foreign Policy." Geopolitics 13 (3): 519-544.

Hartog, Joop, Ada Ferrer-i Carbonell, and Nicole Jonker. 2002. “Linking Measured Risk Aversion to Individual Characteristics." Kyklos 55 (1): 3-26.

Hassner, Ron E. 2003. "'To Halve and to Hold": Conflicts over Sacred Space and the Problem of Indivisibility." Security Studies 12 (4): 1-33.

Hassner, Ron E. 2006/ 2007. “The Path to Intractability: Time and the Entrenchment of Territorial Disputes." International Security 31 (3): 107-138.

Hecht, Tracy D., Natalie J. Allen, Joy D. Klammer, and Elizabeth C. Kelly. 2002. "Group Beliefs, Ability, and Performance: The Potency of Group Potency." Group Dynamics: Theory, Research, and Practice 6 (2): 143-152.

Hedström, Peter, and Petri Ylikoski. 2010. "Causal Mechanisms in the Social Sciences." Annual Review of Sociology 36: 49-67.

Heider, Fritz. 1958. The Psychology of Interpersonal Relations. New York: Wiley.

Henrich, Joseph, Steven J. Heine, and Ana Norenzayan. 2010. "The weirdest people in the world?" Behavioral and Brain Sciences 33 (2-3): 1-75.

Herbert, Bob. 2010. "Resolve Among the Ruins." In The New York Times. p. A21. http://www.nytimes.com/2010/01/16/opinion/16herbert.html.

Hermann, Margaret G., Thomas Preston, Baghat Korany, and Timothy M. Shaw. 2001. "Who Leads Matters: The Effects of Powerful Individuals." International Studies Review 3 (2): 88-131.

Herrmann, Richard. 1988. "The Empirical Challenge of the Cognitive Revolution: A Strategy for the Drawing Inferences about Perceptions." International Studies Quarterly 32 (2): 175-203.

Herrmann, Richard K., James F. Voss, Tonya Y. E. Schooler, and Joseph Ciarrochi. 1997. "Images in International Relations: An Experimental Test of Cognitive Schemata." International Studies Quarterly 41 (3): 403-433.

Herrmann, Richard K., and Michael P. Fischerkeller. 1995. "Beyond the Enemy Image and Spiral Model: Cognitive-Strategic Research After the Cold War." International Organization 49 (3): 415-50.

Herrmann, Richard K., Philip E. Tetlock, and Penny S. Visser. 1999. "Mass Public Decisions to Go to War: A Cognitive-Interactionist Framework." American Political Science Review 93 (3): 553-573. 
Herrmann, Richard K., and Vaughn P. Shannon. 2001. "Defending International Norms: The Role of Obligation, Material Interest, and Perception in Decision Making." International Organization 55 (3): 621-654.

Herrnstein, Richard J. 1970. "On the law of effect." Journal of the Experimental Analysis of Behavior 13 (2): 243-266.

Herron, Michael C. 1999. "Postestimation Uncertainty in Limited Dependent Variable Models." Political Analysis 8 (1): 83-98.

Hoebel, E. Adamson. 1967. "Anthropological Perspectives on National Character." Annals of the American Academy of Political and Social Science 370: 1-7.

Hoffman, Aaron M., Christopher R. Agnew, Justin J. Lehmiller, and Natasha T. Duncan. 2009. "Satisfaction, Alternatives, Investments, and the Microfoundations of Audience Cost Models." International Interactions 35 (4): 365 - 389.

Hoffmann, Tobias. 2008. Weakness of Will From Plato to the Present. Washington, D.C.: The Catholic University of America Press.

Hofmann, Wilhelm, Malte Friese, and Fritz Strack. 2009. “Impulse and Self-Control From a Dual-Systems Perspective." Perspectives on Psychological Science 4 (2): 162176.

Hollis, Martin, and Steve Smith. 1990. Explaining and Understanding International Relations. Oxford: Oxford University Press.

Holsti, Ole R. 2004. Public Opinion and American Foreign Policy. Revised edition ed. Ann Arbor, MI: University of Michigan Press.

Holton, Richard. 1999. "Intention and Weakness of Will." Journal of Philosophy 96 (5): 241-262.

Hopf, Ted. 1991a. "Polarity, the Offense Defense Balance, and War." American Political Science Review 85 (2): 475-493.

Hopf, Ted. 1991b. "Soviet Inferences from Their Victories in the Periphery: Visions of Resistance or Cumulating Gains?" In Dominoes and Bandwagons: Strategic Beliefs and Great Power Competition in the Eurasian Rimland, ed. Robert Jervis and Jack Snyder. Oxford: Oxford University Press.

Hopf, Ted. 1998. "The Promise of Constructivism in International Relations Theory." International Security 23 (1): 171-200.

Hopf, Ted. 2002. Social Construction of International Politics: Identities \& Foreign Policies, Moscow, 1955 \& 1999. Ithaca: Cornell University Press. 
Hopkins, Daniel, and Gary King. 2010. "A Method of Automated Nonparametric Content Analysis for Social Science." American Journal of Political Science 54 (1): 229-247.

Horowitz, Michael C., and Allan C Stam. 2012. "How Prior Military Experience Influences The Future Militarized Behavior of Leaders." Unpublished manuscript.

Horowitz, Michael C., and Matthew S. Levendusky. 2011. "Drafting Support for War: Conscription and Mass Support for Warfare." Journal of Politics 73 (2): 524534 .

Horowitz, Michael, Rose McDermott, and Allan C Stam. 2005. "Leader Age, Regime Type, and Violent International Relations." Journal of Conflict Resolution 49 (5): 661-685.

Hsee, Christopher K., and Elke U. Weber. 1999. “Cross-National Differences in Risk Preference and Lay Predictions." Journal Of Behavioral Decision Making 12 (2): 165179.

Hudson, Valerie M. 2005. "Foreign Policy Analysis: Actor-Specific Theory and the Ground of International Relations." Foreign Policy Analysis 1 (1): 1-30.

Huntington, Samuel P. 1957. The Soldier and the State: The Theory and Politics of CivilMilitary Relations. Cambridge, MA: Belknap/Harvard University Press.

Huntington, Samuel P. 1993. “The Clash of Civilizations?” Foreign Affairs 72: 22-49.

Huth, Paul K. 1997. "Reputations and Deterrence: A Theoretical and Empirical Assessment." Security Studies 7 (1): 72-99.

Iklé, Fred Charles. 1964. How Nations Negotiate. New York: Harper \& Row.

Imai, Kosuke. 2005. "Do Get-Out-the-Vote Calls Reduce Turnout? The Importance of Statistical Methods for Field Experiments." American Political Science Review 99 (2): 283-300.

Imai, Kosuke, Luke Keele, Dustin H. Tingley, and Teppei Yamamoto. 2011. “Unpacking the Black Box of Causality: Learning about Causal Mechanisms from Experimental and Observational Studies." American Political Science Review 105 (November 2011): 765-789.

Iyengar, Radha, and Jonathan Monten. 2008. "Is There an "Emboldenment" Effect? Evidence from the Insurgency in Iraq." NBER Working Paper 13839.

Izjerman, Hans, and Dov Cohen. 2011. "Grounding cultural syndromes: Body comportment and values in honor and dignity cultures." European Journal of Social Psychology 41 (4): 456-467. 
Jabri, Vivienne, and Stephen Chan. 1996. "The ontologist always rings twice: two more stories about structure and agency in reply to Hollis and Smith." Review of International Studies 22 (1): 107-110.

Jaccard, James, and Robert Turrisi. 2003. Interaction effects in multiple regression. Vol. 72 of University Paper series on Quantitative Applications in the Social Sciences 2nd ed. Newbury Park: Sage.

Jentleson, Bruce W. 1992. “The Pretty Prudent Public: Post Post-Vietnam American Opinion on the Use of Military Force." International Studies Quarterly 36 (1): 49-74.

Jervis, Robert. 1972. "Bargaining and Bargaining Tactics." In Nomos, vol. 14, Coercion, ed. J. Roland Pennock and John W. Chapman. Vol. 14. Chicago: Aldine Atherton.

Jervis, Robert. 1976. Perception and Misperception in International Politics. Princeton, NJ: Princeton University Press.

Jervis, Robert. 1979. “Deterrence Theory Revisited.” World Politics 31: 289-324.

Jervis, Robert. 2013. "Do Leaders Matter and How Would We Know?" Security Studies 22 (2): 153-179.

Johnsen, Bjørn Helge, Jarle Eid, Staale Pallesen, Paul T. Bartone, and Odd A. Nissestad. 2009. "Predicting Transformational Leadership in Naval Cadets: Effects of Personality Hardiness and Training." Journal of Applied Social Psychology 39 (9): 2213-2235.

Johnson, Dominic D. P., and Dominic Tierney. 2006. Failing to Win: Perceptions of Victory and Defeat in International Politics. Cambridge, MA: Harvard University Press.

Johnson, Dominic D. P., Richard W. Wrangham, and Stephen Peter Rosen. 2002. “Is military incompetence adaptive? An empirical test with risk-taking behaviour in modern warfare." Evolution and Human Behavior 23: 245-264.

Johnson, Dominic D. P., Rose McDermott, Emily S. Barrett, Jonathan Cowden, Richard Wrangham, Matthew H. McIntyre, and Stephen Peter Rosen. 2006. "Overconfidence in wargames: experimental evidence on expectations, aggression, gender and testosterone." Proceedings of the Royal Society B: Biological Sciences 273 (1600): 2513-2520.

Johnston, Alastair Iain. 1995. Cultural Realism: Strategic Culture and Grand Strategy in Chinese History. Princeton, NJ: Princeton University Press.

Jolls, Christine, Cass R. Sunstein, and Richard Thaler. 1998. "A Behavioral Approach to Law and Economics." Stanford Law Review 50 (5): 1471-1550. 
Joseph, Jonathan. 2013. "Resilience in UK and French Security Strategy: An AngloSaxon Bias?" Politics Forthcoming.

Joseph, Jonathan, and Colin Wight. 2010. Scientific Realism and International Relations. New York: Palgrave Macmillan.

Kahneman, Daniel, and Amos Tversky. 2000. Choices, Values and Frames. Cambridge: Cambridge University Press.

Kalis, Annemarie, Andreas Mojzisch, T. Sophie Schweizer, and Stefan Kaiser. 2008. "Weakness of will, akrasia, and the neuropsychiatry of decision making: An interdisciplinary perspective." Cognitive, Affective \& Behavioral Neuroscience 8 (4): $402-417$.

Kam, Cindy D., and Elizabeth N. Simas. 2010. "Risk Orientations and Policy Frames." Journal of Politics 72 (2): 381-396.

Kam, Cindy D., and Jennifer M. Ramos. 2008. "Joining and Leaving the Rally: Understanding the Surge and Decline in Presidential Approval Following 9/11." Public Opinion Quarterly 72 (4): 619-650.

Katzenstein, Peter J. 1996. The Culture of National Security: Norms and Identity in World Politics. New York: Columbia University Press.

Keele, Luke. 2008. Semiparametric Regression for the Social Sciences. Chichester, UK: John Wiley \& Sons.

Keele, Luke J. 2010. “Proportionally Difficult: Testing for Nonproportional Hazards in Cox Models." Political Analysis 18 (2): 189-205.

Kennedy, Paul. 1987. The Rise and Fall of the Great Powers. New York: Vintage Books.

Keohane, Robert O., and Jr. Nye, Joseph S. 1977. Power and Interdependence. New York: HarperCollins.

Kertzer, Joshua D. 2013. "Making Sense of Isolationism: Foreign Policy Mood as a Multilevel Phenomenon." Journal of Politics 75 (1): 225-240.

Kertzer, Joshua D., and Kathleen M. McGraw. 2010. “Uncertainty, Attributions and Foreign Policy Preferences: A Fractional Factorial Experiment." In American Political Science Association annual meeting, . Washington, D.C.

Kertzer, Joshua D., and Kathleen M. McGraw. 2012. "Folk Realism: Testing the Microfoundations of Realism in Ordinary Citizens." International Studies Quarterly 56 (2): 245-258.

Khong, Yuen Foong. 1992. Analogies at War: Korea, Munich, Dien Bien Phu and the Vietnam Decision of 1965. Princeton, NJ: Princeton University Press. 
Kim-Cohen, J, A Caspi, A Taylor, B Williams, R Newcombe, IW Craig, and TE Moffitt. 2006. "MAOA, maltreatment, and gene-environment interaction predicting children's mental health: new evidence and a meta-analysis." Molecular Psychiatry 11: 903-913.

King, Gary. 1991. "“Truth” Is Stranger than Prediction, More Questionable than Causal Inference." American Journal of Political Science 35 (4): 1047-1053.

King, Gary, Jenifer Pan, and Margaret E. Roberts. 2013. "How Censorship in China Allows Government Criticism but Silences Collective Expression." American Political Science Review 107 (2): 1-18.

King, Gary, and Langche Zeng. 2001. "Explaining Rare Events in International Relations." International Organization 55 (3): 693-715.

King, Gary, and Langche Zeng. 2007. "When Can History Be Our Guide? The Pitfalls of Counterfactual Inference." International Studies Quarterly 51 (1): 183-210.

King, Lynda A., Daniel W. King, John A. Fairbank, Terence M. Keane, and Gary A. Adams. 1998. "Resilience-Recovery Factors in Post-Traumatic Stress Disorder Among Female and Male Vietnam Veterans: Hardiness, Postwar Social Support, and Additional Stressful Life Events." Journal of Personality and Social Psychology 74 (2): 420-434.

Kirshner, Jonathan. 2000. "Rationalist Explanations for War?" Security Studies 10 (1): 143-150.

Kivetz, Ran, and Anat Keinan. 2006. "Repenting Hyperopia: An Analysis of SelfControl Regrets." Journal of Consumer Research 33 (2): 273-282.

Kivetz, Ran, and Itamar Simonson. 2002. "Self-Control for the Righteous: Toward a Theory of Precommitment to Indulgence." Journal of Consumer Research 29 (2): 199-217.

Klarevas, Louis. 2002. "The "Essential Domino" of Military Operations: American Public Opinion and the Use of Force." International Studies Perspectives 3 (4): 417437.

Klein, William M., and Ziva Kunda. 1993. "Maintaining Self-Serving Social Comparisons: Biased Reconstruction of One's Past Behaviors." Personality and Social Psychology Bulletin 19 (6): 732-739.

Klingberg, Frank L. 1952. "The Historical Alternation of Moods in American Foreign Policy." World Politics 4 (2): 239-273.

Koch, Michael, and Scott Sigmund Gartner. 2005. "Casualties and Constituencies: Democratic Accountability, Electoral Institutions, and Costly Conflicts." Journal of Conflict Resolution 49 (6): 874-894. 
Koch, Michael T., and Patricia L. Sullivan. 2010. "Should I Stay or Should I Go Now? Partisanship, Approval, and the Duration of Major Power Democratic Military Interventions." Journal of Politics 72 (3): 616-629.

Kohn, Alfie. 2008. "Why Self-Discipline is Overrated: The (Troubling) Theory and Practice of Control from Within." Phi Delta Kappan 90 (3): 168-176.

Kowert, Paul A., and Margaret G. Hermann. 1997. "Who Takes Risks? Daring and Caution in Foreign Policy Making." Journal of Conflict Resolution 41 (5): 611-637.

Krauthammer, Charles. 2009. "Decline is a Choice: The New Liberalism and the end of American ascendancy." The Weekly Standard 15 (October 19, 2009).

Krebs, Ronald R., and Aaron Rapport. 2012. "International Relations and the Psychology of Time Horizons." International Studies Quarterly Forthcoming.

Krebs, Ronald R., and Patrick Thaddeus Jackson. 2007. "Twisting Tongues and Twisting Arms: The Power of Political Rhetoric." European Journal of International Relations 13 (1): 35-66.

Kreps, Sarah. 2008. "When Does the Mission Determine the Coalition? The Logic of Multilateral Intervention and the Case of Afghanistan." Security Studies 17 (3): 531-567.

Kuhl, Julius. 1988. "Functional characteristics of human self-control." Behavioral and Brain Sciences 11 (4): 688.

Kunda, Ziva. 1990. “The Case for Motivated Reasoning." Psychological Bulletin 108 (3): 480-498.

Kydd, Andrew. 2003. "Which Side Are You On? Bias, Credibility, and Mediation." American Journal of Political Science 47 (4): 597-611.

Kydland, Finn E., and Edward C. Prescott. 1977. "Rules Rather than Discretion: The Inconsistency of Optimal Plans." Journal of Political Economy 85 (3): 473-492.

Laffont, Jean-Jacques, and David Martimort. 2002. The Theory of Incentives: The Principal-Agent Model. Princeton, NJ: Princeton University Press.

Lake, David A. 2010/11. “Two Cheers for Bargaining Theory: Assessing Rationalist Explanations of the Iraq War." International Security 35 (3): 7-52.

Lake, David A. 2011. "Why "isms" are Evil: Theory, Epistemology, and Academic Sects as Impediments to Understanding and Progress." International Studies Quarterly 55 (2): 465-480.

Langlois, Catherine C., and Jean-Pierre P. Langlois. 2009. “Does Attrition Behavior Help Explain the Duration of Interstate Wars? A Game Theoretic and Empirical Analysis." International Studies Quarterly 53 (4): 1051-1073. 
Larson, Eric V. 2000. "Putting Theory to Work: Diagnosing Public Opinion on the U.S. Intervention in Bosnia." In Being Useful: Policy Relevance and International Relations Theory, ed. Miroslav Nincic and Joseph Lepgold. Ann Arbor: University of Michigan Press.

Lau, Ursula, and Ashley van Niekerk. 2011. "Restorying the Self: An Exploration of Young Burn Survivors' Narratives of Resilience." Qualitative Health Research 21 (9): 1165-1181.

Lebow, Richard Ned. 1998. "Beyond Parsimony: Rethinking Theories of Coercive Bargaining." European Journal of International Relations 4 (1): 31-66.

Leeds, Brett Ashley, Jeffrey M. Ritter, Sara McLaughlin Mitchell, and Andrew G. Long. 2002. "Alliance Treaty Obligations and Provisions, 1815-1944." International Interactions 28: 237-260.

Leites, Nathan. 1951. The Operational code of the Politburo. McGraw-Hill.

Lemke, Thomas. 2001. "'The birth of bio-politics': Michel Foucault's lecture at the College de France on neo-liberal governmentality." Economy and Society 30 (2): 190-207.

Lentz, Harris M. 1994. Heads of States and Governments: A Worldwide Encyclopedia of Over 2,300 Leaders, 1945 through 1992. Jefferson, NC: McFarland \& Company.

Leung, Angela K.-Y., and Dov Cohen. 2011. “Within- and Between-Culture Variation: Individual Differences and the Cultural Logics of Honor, Face, and Dignity Cultures." Journal of Personality and Social Psychology 100 (3): 507-526.

Levendusky, Matthew S., and Michael C. Horowitz. 2012. "When Backing Down is the Right Decision: Partisanship, New Information, and Audience Costs." Journal of Politics 74 (2): 323-338.

Levy, Charles S. 1999. "Implementing TRIPS - A Test of Political Will." Law and Policy in International Business 31: 789-795.

Levy, Jack S. 1997. "Prospect Theory, Rational Choice, and International Relations." International Studies Quarterly 41 (1): 87-112.

Levy, Jack S., and William F. Mabe Jr. 2004. "Politically Motivated Opposition to War." International Studies Review 6 (1): 65-83.

Levy, Yael. 2006. "The War of the Peripheries: A Social Mapping of IDF Casualties in the Al-Aqsa Intifada." Social Identities 12 (3): 309-324.

Lewin, Eyal. 2012. National Resilience During War: Refining the Decision-Making Model. Plymouth, UK: Lexington Books. 
Lieberman, Joseph. 2011. “A Decade of Resolve." In Foreign Policy. http://www. foreignpolicy.com/articles/2011/09/09/a_decade_of_resolve.

Loehlin, John C. 1992. Genes and environment in personality development. Newbury Park, CA: Sage.

Loewenstein, George, and Jon Elster. 1992. Choice Over Time. New York: Russell Sage Foundation.

Logue, A.W. 1988. "Research on self-control: An integrating framework." Behavioral and Brain Sciences 11 (4): 665-709.

Logue, A.W. 1998. "Self-Control." In Learning and Behavior Therapy, ed. William O'Donohue. Boston: Allyn and Bacon.

Lupovici, Amir. 2010. “The Emerging Fourth Wave of Deterrence Theory - Toward a New Research Agenda." International Studies Quarterly 54 (3): 705-732.

Lyall, Jason. 2010. “Do Democracies Make Inferior Counterinsurgents? Reassessing Democracy's Impact on War Outcomes and Duration." International Organization 64 (1): 167-92.

Lyall, Jason, Graeme Blair, and Kosuke Imai. Forthcoming. "Explaining Support for Combatants during Wartime: A Survey Experiment in Afghanistan." American Political Science Review.

MacCrimmon, Kenneth R., Donald A. Wehrung, and W.T. Stanbury. 1986. Taking risks: The management of uncertainty. New York: Free Press.

Mack, Andrew. 1974. "Why Big Nations Lose Small Wars: The Politics of Asymmetric Conflict." World Politics 27 (2): 175-200.

MacKinnon, David P., Jennifer L. Krull, and Chondra M. Lockwood. 2000. “Equivalence of the Mediation, Confounding and Suppression Effect." Prevention Science 1 (4): 173-181.

Magaletta, Philip R., and J.M. Oliver. 1999. "The Hope Construct, Will, and Ways: Their Relations with Self-Efficacy, Optimism, and General Well-Being." Journal Of Clinical Psychology 55 (5): 539-551.

Manley, John, Derek H. Burney, Jake Epp, Paul Tellier, and Pamela Wallin. 2008. Independent Panel on Canada's Future Role in Afghanistan. Technical report Minister of Public Works.

Mansfield, Edward D., and Jack Snyder. 1995. "Democratization and the Danger of War." International Security 20 (1): 5-38.

Maoz, Zeev. 1983. "Resolve, Capabilities, and the Outcomes of Interstate Disputes, 1816-1976." Journal of Conflict Resolution 27 (2): 195-229. 
Maoz, Zeev, and Randolph M. Siverson. 2008. "Bargaining, Domestic Politics, and International Context in the Management of War: A Review Essay." Conflict Management and Peace Science 25 (2): 171-189.

March, James G. 1966. “The Power of Power." In Varieties of Political Theory, ed. David Easton. Englewood Cliffs, N.J.: Prentice-Hall.

March, James G., and Johan P. Olsen. 1998. "The Institutional Dynamics of International Political Orders." International Organization 52 (4): 943-969.

Martin, Lisa L. 1999. "The Contributions of Rational Choice: A Defense of Pluralism." International Security 24 (2): 74-83.

Mattes, Michaela, and T. Clifton Morgan. 2004. "When Do They Stop? Modeling the Termination of War." Conflict Management and Peace Science 21 (3): 179-193.

Mattiacci, Eleonora, and Bear F. Braumoeller. 2012. "The Fog of Peace: Uncertainty, War and the Resumption of International Crises." Unpublished manuscript, The Ohio State University.

May, Joshua, and Richard Holton. 2012. "What in the World is Weakness of Will?" Philosophical Studies 157: 341-360.

McCrae, Robert R., and Antonio Terracciano. 2005. "Personality Profiles of Cultures: Aggregate Personality Traits." Journal of Personality and Social Psychology 89 (3): 407-425.

McCrae, Robert R., and Jr. Costa, Paul T. 1987. "Validation of the Five-Factor Model of Personality Across Instruments and Observers." Journal of Personality and Social Psychology 52 (1): 81-90.

McDermott, Rose. 1998. Risk-Taking in International Politics: Prospect Theory in American Foreign Policy. University of Michigan Press.

McDermott, Rose, and Jonathan A. Cowden. 2001. "The effects of uncertainty and sex in a crisis simulation game." International Interactions 27 (4): 353-380.

Mead, Walter Russell. 2002. Special Providence: American Foreign Policy and How it Changed the World. New York: Routledge.

Mearsheimer, John J. 2001. The Tragedy of Great Power Politics. New York: W.W. Norton.

Mebane, Walter R. Jr., and Jasjeet S. Sekhon. 2011. “Genetic Optimization Using Derivatives: The rgenoud package for R." Journal of Statistical Software 42 (11).

Meirowitz, Adam, and Ann E. Sartori. 2008. "Strategic Uncertainty as a Cause of War." Quarterly Journal of Political Science 3 (4): 327-352. 
Mercer, Jonathan. 1996. Reputation and International Politics. Ithaca, NY: Cornell University Press.

Mercer, Jonathan. 2010. “Emotional Beliefs.” International Organization 64 (1): 1-31.

Merom, Gil. 2003. How Democracies Lose Small Wars: State, Society, and the Failures of France in Algeria, Israel in Lebanon, and the United States in Vietnam. Cambridge: Cambridge University Press.

Merton, Robert K. 1968. Social Theory and Social Structure. Free Press.

Michaels, Jim, and Theodore May. 2011. "Protesters' resolve 'unshakable': Many promise to continue demonstrations until Mubarak has left office." In USA Today. p. 5A. http://usatoday30.usatoday.com/printedition/news/20110202/ egyptinside02_st.art.htm.

Miller, Holly C., Kristina F. Pattison, C. Nathan DeWall, Rebecca Rayburn-Reeves, and Thomas R. Zentall. 2010. "Self-Control Without a "Self"?" Psychological Science: $1-5$.

Miller, John H., and Scott E. Page. 2007. Complex Adaptive Systems: An Introduction to Computational Models of Social Life. Princeton: Princeton University Press.

Miller, Ted R. 2000. "Variations between Countries in Values of Statistical Life." Journal of Transport Economics and Policy 34 (2): 169-188.

Milner, Helen. 1997. Interests, Institutions and Information: Domestic Politics and International Relations. Princeton, NJ: Princeton University Press.

Mintz, Alex, Steven B. Redd, and Arnold Vedlitz. 2006. "Can We Generalize from Student Experiments to the Real World in Political Science, Military Affairs, and International Relations?" Journal of Conflict Resolution 50 (5): 757-776.

Mischel, Walter. 1968. Personality and Assessment. New York: Wiley.

Mischel, Walter. 2004. "Toward an Integrative Science of the Person." Annual Review of Psychology 55: 1-22.

Mischel, Walter, and Joan Grusec. 1967. "Waiting for Rewards and Punishments: Effects of Time and Probability on Choice." Journal of Personality and Social Psychology 5 (1): 24-31.

Mischel, Walter, Yuichi Shoda, and Monica L. Rodriguez. 1989. “Delay of Gratification in Children." Science 244 (4907): 933-938.

Moller, Arlen C., Edward L. Deci, and Richard M. Ryan. 2006. "Choice and EgoDepletion: The Moderating Role of Autonomy." Personality and Social Psychology Bulletin 32 (8): 1024-1036. 
Mondak, Jeffrey J. 2010. Personality and the Foundations of Political Behavior. Cambridge: Cambridge University Press.

Moore, Jay. 1988. "Evolution and impulsiveness." Behavioral and Brain Sciences 11 (4): 691.

Moravscik, Andrew. 1997. "Taking Preferences Seriously: A Liberal Theory of International Politics." International Organization 51 (4): 513-53.

Morgan, T. Clifton. 1990. "Power, Resolve and Bargaining in International Crises: A Spatial Theory." International Interactions 15 (3/4): 279-302.

Morgan, T. Clifton. 1994. Untying the Knot of War: A Bargaining Theory of International Crises. Ann Arbor: University of Michigan Press.

Morgenthau, Hans J. 1951. In Defense of the National Interest. New York: Alfred A. Knopf.

Morgenthau, Hans J. 1985. Politics Among Nations: The Struggle for Power and Peace. Brief ed. Boston, MA: McGraw-Hill.

Morrow, James D. 1985. "A Continuous-Outcome Expected Utility Theory of War." Journal of Conflict Resolution 29 (3): 473-502.

Morrow, James D. 1987. "On the Theoretical Basis of a Measure of National Risk Attitudes." International Studies Quarterly 31 (4): 423-438.

Morrow, James D. 1988. "Social Choice and System Structure in World Politics." World Politics 41 (1): 75-97.

Morrow, James D. 1989. “Capabilities, Uncertainty, and Resolve: A Limited Information Model of Crisis Bargaining." American Journal of Political Science 33 (4): 941-72.

Morsella, Ezequiel, John A. Bargh, and Peter M. Gollwitzer. 2009. Oxford Handbook of Human Action. Oxford: Oxford University Press.

Morton, Rebecca B., and Kenneth C. Williams. 2010. Expeirmental Political Science and the Study of Causality. Cambridge University Press.

Most, Benjamin A., and Harvey Starr. 1984. "International Relations Theory, Foreign Policy Substitutability, and “Nice” Laws." World Politics 36 (3): 383-406.

Most, Benjamin A., and Harvey Starr. 1989. Inquiry, Logic and International Politics. Columbia, SC: University of South Carolina Press.

Mueller, John. 2005. “The Iraq Syndrome.” Foreign Affairs 84: 44-54.

Mueller, John E. 1971. "Trends in Popular Support for the Wars in Korea and Vietnam." American Political Science Review 65 (2): 358-375. 
Mueller, John E. 1980. "The Search for the "Breaking Point" in Vietnam: The Statistics of a Deadly Quarrel." International Studies Quarterly 24 (4): 497-519.

Müller, Harald. 2004. "Arguing, Bargaining and All That: Communicative Action, Rationalist Theory and the Logic of Appropriateness in International Relations." European Journal of International Relations 10 (3): 395-435.

Muraven, Mark, R. Lorraine Collins, Saul Shiffman, and Jean A. Paty. 2005. "Daily Fluctuations in Self-Control Demands and Alcohol Intake." Psychology of Addictive Behaviors 19 (2): 140-147.

Mutz, Diana C. 2011. Population-Based Survey Experiments. Princeton University Press.

Myers, Teresa A., and Andrew F. Hayes. 2010. "Reframing the Casualties Hypothesis: (Mis)perceptions of Troop Loss and Public Opinion about War." International Journal of Public Opinion Research 22 (2): 256-275.

Nelson, Alan. 1984. "Some Issues Surrounding the Reduction of Macroeconomics to Microeconomics." Philosophy of Science 51 (4): 573-594.

Nincic, Miroslav. 1997. "Domestic Costs, the U.S. Public, and the Isolationist Calculus." International Studies Quarterly 41 (4): 593-610.

Nisbett, Richard E., and Dov Cohen. 1996. Culture of Honor: The Psychology of Violence in the South. Boulder, CO: Westview Press.

Noyes, Richard. 1980. “The Time Horizon of Planned Social Change: I. Why Utopian Movements Always Promise Amelioration in the Future." American Journal of Economics and Sociology 39 (1): 65-77.

Offer, Avner. 2006. The Challenge of Affluence: Self-Control and Well-Being in the United States and Britain since 1950. Oxford: Oxford University Press.

O'Neill, Barry. 1999. Honor, Symbols and War. Ann Arbor: University of Michigan Press.

O'Neill, Barry. 2001. “Risk Aversion in International Relations Theory." International Studies Quarterly 45 (4): 617-649.

Ong, Anthony D., C.S. Bergeman, Toni L. Bisconti, and Kimberly A. Wallace. 2006. "Psychological Resilience, ositive Emotions, and Successful Adaptation to Stress in Later Life." Journal of Personality and Social Psychology 91 (4): 730-749.

Onuf, Nicholas. 1995. "Levels." European Journal of International Relations 1 (1): 3558.

Organski, A.F.K. 1968. World Politics. 2nd ed. New York: Alfried A. Knopf. 
Orne, Martin T. 1962. "On the social psychology of the psychological expeirment: With particular reference to demand characteristics and their implications." American Psychologist 17 (11): 776-783.

Osterman, Lindsey L., and Ryan P. Brown. 2011. "Culture of Honor and Violence Against the Self." Personality and Social Psychology Bulletin 37 (12): 1611-1623.

Oye, Kenneth A. 1985. "Explaining Cooperation Under Anarchy: Hypotheses and Strategies." World Politics 38 (1): 1-24.

Page, Benjamin I., and Robert Y. Shapiro. 1992. The Rational Public: Fifty Years of Trends in Americans' Policy Preferences. Chicago: University of Chicago Press.

Palacios-Huerta, Ignacio. 2003. “Time-Inconsistent Preferences in Adam Smith and David Hume." History of Political Economy 35 (2): 241-268.

Palmer, Glenn, Tamar London, and Patrick Regan. 2004. “What's stopping you? The sources of political constraints on international conflict behavior in parliamentary democracies." International Interactions 30: 1-24.

Pape, Robert A. 1996. Bombing to Win: Air Power and Coercion in War. Ithaca, NY: Cornell University Press.

Pape, Robert A. 2003. “The Strategic Logic of Suicide Terrorism." American Political Science Review 97 (3): 343-361.

Papies, Esther K., Wolfgang Stroebe, and Henk Aarts. 2008. "Healthy Cognition: Processes of Self-Regulatory Success in Restrained Eating." Personality and Social Psychology Bulletin 34 (9): 1290-1300.

Parfit, Derek. 1971. "Personal Identity." The Philosophical Review 80 (1): 3-27.

Payne, Rodger A. 2001. "Persuasion, Frames and Norm Construction." European Journal of International Relations 7 (1): 37-61.

Perri, Michael G., C. Steven Richards, and Karen R. Schultheis. 1977. “Behavioral self-control and smoking reduction: A study of self-initiated attempts to reduce smoking." Behavior Therapy 8 (3): 360-365.

Polsky, Andrew J. 2010. "Staying the Course: Presidential Leadership, Military Stalemate, and Strategic Inertia." Perspectives on Politics 8 (1): 127-139.

Posner, Richard A. 1997. "Are We One Self or Multiple Selves? Implications for Law and Public Policy." Legal Theory 3 (1): 23-35.

Powell, Robert. 1987. "Crisis Bargaining, Escalation, and MAD." American Political Science Review 81 (3): 717-735. 
Powell, Robert. 1988. “Nuclear Brinksmanship with Two-Sided Incomplete Information." American Political Science Review 82 (1): 155-177.

Powell, Robert. 2003. “Nuclear Deterrence Theory, Nuclear Proliferation, and National Missile Defense." International Security 27 (4): 86-118.

Powell, Robert. 2004. "Bargaining and Learning While Fighting." American Journal of Political Science 48 (2): 344-361.

Powell, Robert. 2006. "War as a Commitment Problem." International Organization 60 (1): 169-203.

Power, Samantha. 2002. "A Problem from Hell": America and the Age of Genocide. New York: Basic Books.

Pratt, Travis C., Michael G. Turner, and Alex R. Piquero. 2004. “Parental Socialization and Community Context: A Longitudinal Analysis of the Structural Sources of Low Self-Control." Journal of Research in Crime and Delinquency 41 (3): 219-243.

Press, Daryl G. 2005. Calculating Credibility: How Leaders Assess Military Threats. Ithaca, NY: Cornell University Press.

Presser, Stanley, and Howard Schuman. 1981. Questions and Answers in Attitude Surveys: Experiments on Question Form, Wording, and Context. San Diego: Academic Press.

Przeworski, Adam, and James Raymond Vreeland. 2000. "The effect of IMF programs on economic growth." Journal of Development Economics 62: 385-421.

Putnam, Robert D. 1988. “Diplomacy and Domestic Politics: the Logic of Two-Level Games." International Organization 42 (3): 427-60.

Rachlin, Howard, and Leonard Green. 1972. "Commitment, Choice and SelfControl." Journal of the Experimental Analysis of Behavior 17 (1): 15-22.

Raftery, Adrian E. 1995. "Bayesian Model Selection in Social Research." Sociological Methodology 25: 111-164.

Ramsay, Kristopher W. 2008. "Settling It on the Field: Battlefield Events and War Termination." Journal of Conflict Resolution 52 (6): 850-879.

Rauchhaus, Robert W. 2006. "Asymmetric Information, Mediation, and Conflict Management." World Politics 58 (2): 207-241.

Ray, James Lee, and Ayse Vural. 1986. "Power Disparities and Paradoxical Conflict Outcomes." International Interactions 12 (4): 315-342.

Read, Daniel. 2006. "Which side are you on? The ethics of self-command." Journal of Economic Psychology 27 (5): 681-693. 
Recouly, Raymond. 1920. Foch: The Winner of the War. Charles Scribner's Sons.

Reed, William. 2000. "A Unified Statistical Model of Conflict Onset and Escalation." American Journal of Political Science 44 (1): 84-93.

Reed, William. 2003. "Information, Power, and War." American Political Science Review 97 (4): 633-641.

Reiter, Dan, and Allan C Stam. 2002. Democracies at War. Princeton, NJ: Princeton University Press.

Reiter, Dan, and III Stam, Allan C. 1998a. "Democracy and Battlefield Military Effectiveness." Journal of Conflict Resolution 42 (3): 259-277.

Reiter, Dan, and III Stam, Allan C. 1998b. "Democracy, War Initiation, and Victory." American Political Science Review 92 (2): 377-389.

Renshon, Jonathan. 2008. "Stability and Change in Belief Systems: The Operational Code of George W. Bush." Journal of Conflict Resolution 52 (6): 820-849.

Renshon, Jonathan. 2009. “When Public Statements Reveal Private Beliefs: Assessing Operational Codes at a Distance." Political Psychology 30 (4): 649-661.

Renshon, Jonathan, and Arthur Spirling. Forthcoming. "Modeling 'Effectiveness' in International Relations." Journal of Conflict Resolution.

Rentfrow, Peter J., Samuel D. Gosling, and Jeff Potter. 2008. "A Theory of the Emergence, Persistence, and Expression of Geographic Variation in Psychological Characteristics." Perspectives on Psychological Science 3 (5): 339-369.

Reus-Smit, Christian. 2003. "Politics and International Legal Obligation." European Journal of International Relations 9 (4): 591-625.

Reynolds, Katherine J., John C. Turner, Nyla R. Branscombe, Kenneth I. Mavor, Boris Bizumic, and Emina Subašić. 2010. "Interactionism in personality and social psychology: An integrated approach to understanding the mind and behaviour." European Journal of Personality 24 (5): 458-482.

Richardson, Lewis F. 1948. "Variation of the frequency of fatal quarrels with magnitude." Journal of the American Statistical Association 43 (244): 523-546.

Roberts, Brent W., Oleksandr S. Chernyshenko, Stephen Stark, and Lewis R. Goldberg. 2005. "The Structure of Conscientiousness: An Empirical Investigation Based on Seven Major Personality Questionnaires." Personnel Psychology 58: 103139.

Rodriguez Mosquera, Patricia M., Antony S.R. Manstead, and Agneta H. Fischer. 2002. "The role of honour concerns in emotional reactions to offences." Cognition and Emotion 16 (1): 143-163. 
Rogers, Alan R. 1994. "Evolution of Time Preference by Natural Selection." American Economic Review 84 (3): 460-481.

Rosato, Sebastian. 2003. “The Flawed Logic of Democratic Peace Theory." American Political Science Review 97 (4).

Rose, Gideon. 1998. "Neoclassical Realism and Theories of Foreign Policy." World Politics 51 (1): 144-172.

Rosen, Stephen. 1972. "War Power and the Willingness to Suffer." In Peace, War, and Numbers, ed. Bruce M. Russett. Beverly Hills, CA: Sage Publications.

Rosen, Stephen Peter. 2005. War and Human Nature. Princeton, NJ: Princeton University Press.

Rosenau, James N. 1980. “Thinking Theory Thoroughly." In The Scientific Study of Foreign Policy. Revised \& enlarged ed. London: Frances Pinter Publishers.

Ross, Lee. 1977. "The intuitive psychologist and his shortcomings: Distortions in the attribution process." In Advances in Experimental Social Psychology, Vol. 10, ed. Leonard Berkowitz. New York: Academic Press.

Rubinstein, Ariel. 1982. "Perfect Equilibrium in a Bargaining Model." Econometrica 50 (1): 97-109.

Rummel, Rudolph J. 1975a. Understanding Conflict and War: Vol. 1, The Dynamic Psychological Field. Beverly Hills, CA: Sage Publications.

Rummel, Rudolph J. 1975b. Understanding Conflict and War: Vol. 4, War, Power, Peace. Beverly Hills, CA: Sage Publications.

Russett, Bruce, Jonathan Cowden, David Kinsella, and Shoon Murray. 1994. “Did Americans' Expectations of Nuclear War Reduce Their Savings?" International Studies Quarterly 38 (4): 587-603.

Russett, Bruce, and Miles Lackey. 1987. "In the Shadow of the Cloud: If There's No Tomorrow, Why Save Today?" Political Science Quarterly 102 (2): 259-272.

Russett, Bruce, and Miroslav Nincic. 1976. "American Opinion on the Use of Military Force Abroad." Political Science Quarterly 91 (3): 411-431.

Ryan, Richard M., and Edward L. Deci. 2006. "Self-Regulation and the Problem of Human Autonomy: Does Psychology Need Choice, Self-Determination, and Will?" Journal of Personality 74 (6): 1557-1586.

Sarason, Irwin G., Ronald E. Smith, and Edward Diener. 1975. "Personality Research: Components of Variance Attributable to the Person and the Situation." Journal of Personality and Social Psychology 32 (2): 199-204. 
Sartori, Ann E. 2002. "The Might of the Pen: A Reputational Theory of Communication in International Disputes." International Organization 56 (1): 121-149.

Sartori, Ann E. 2005. Deterrence by Dipomacy. Princeton, NJ: Princeton University Press.

Sartori, Giovanni. 1970. "Concept Misformation in Comparative Politics." American Political Science Review 64 (4): 1033-1053.

Saunders, Elizabeth N. 2011. Leaders at War: How Presidents Shape Military Interventions. Ithaca, NY: Cornell University Press.

Schaller, Mark, and Damian R. Murray. 2008. "Pathogens, Personality, and Culture: Disease Prevalence Predicts Worldwide Variability in Sociosexuality, Extraversion, and Openness to Experience." Journal of Personality and Social Psychology 95 (1): 212-221.

Schelling, Thomas C. 1960. The Strategy of Conflict. Cambridge, MA: Harvard University Press.

Schelling, Thomas C. 1966. Arms and Influence. New Haven, CT: Yale University Press.

Schelling, Thomas C. 1978. Micromotives and Macrobehavior. New York: W.W. Norton.

Schelling, Thomas C. 1984. "Self-Command in Practice, in Policy, and in a Theory of Rational Choice." American Economic Review 74 (2): 1-11.

Schmitt, Manfred. 2009. "Person x situation-interactions as moderators." Journal of Research in Personality 43 (2): 267.

Schneider, Gerald, Nils Petter Gleditsch, and Sabine Carey. 2011. "Forecasting in International Relations: One Quest, Three Approaches." Conflict Management and Peace Science 28 (1): 5-14.

Schultz, Kenneth. 1998. "Domestic Opposition and Signaling in International Crises." American Political Science Review 92 (4): 829-44.

Schultz, Kenneth A. 1999. “Do Democratic Institutions Constrain or Inform? Contrasting Two Institutional Perspectives on Democracy and War." International Organization 53 (2): 233-266.

Schultz, Kenneth A. 2001a. Democracy and Coercive Diplomacy. New York: Cambridge University Press.

Schultz, Kenneth A. 2001b. "Looking for Audience Costs." Journal of Conflict Resolution 45 (1): 32-60. 
Schuman, Howard. 1992. "Historical Analogies, Generational Effects, and Attitudes Toward War." American Sociological Review 57 (3): 315-326.

Schwartz, Shalom H. 1992. “Universals in the Content and Structure of Values: Theoretical Advances and Empirical Tests in 20 Countries." In Advances in Experimental Social Psychology, Vol. 25, ed. Mark P. Zanna. Vol. 25. San Diego: Academic Press.

Schweller, Randall L. 1994. "Bandwagoning for Profit: Bringing the Revisionist State Back In." International Security 19 (1): 72-107. The MIT Press

Schweller, Randall L. 2003. “The Progressiveness of Neoclassical Realism." In Progress in International Relations Theory: Appraising the Field, ed. Colin Elman and Miriam Fendius Elman. Cambridge, MA: MIT Press.

Schweller, Randall L. 2006. Unanswered Threats: Political Constraints on the Balance of Power. Princeton, NJ: Princeton University Press.

Sechser, Todd S. 2004. "Are Soldiers Less War-Prone than Statesmen?" Journal of Conflict Resolution 48 (5): 746-774.

Shadish, William R., Thomas D. Cook, and Donald T. Campbell. 2002. Experimental and Quasi-Experimental Designs for Generalized Causal Inference. Boston: HoughtonMifflin.

Shannon, Vaughn P., and Michael Dennis. 2007. "Militant Islam and the Futile Fight for Reputation." Security Studies 16 (2): 287-317.

Sheeran, Paschal. 2002. "Intention-Behavior Relations: A Conceptual and Empirical Review." In European Review of Social Psychology, ed. Wolfgang Stroebe and Miles Hewstone. Vol. 12. London: John Wiley \& Sons.

Shepsle, Kenneth A., and Barry R. Weingast. 1981. "Structure-induced equilibrium and legislative choice." Public Choice 37: 503-519.

Shils, Edward A., and Morris Janowitz. 1948. "Cohesion and Disintegration in the Wehrmacht in World War II." Public Opinion Quarterly 12 (2): 280-315.

Simmons, Beth A., Frank Dobbin, and Geoffrey Garrett. 2006. "Introduction: The International Diffusion of Liberalism." International Organization 60 (4): 781-810.

Simon, Herbert A. 1957. Models of man. New York: Wiley.

Simon, Herbert A. 1985. "Human Nature in Politics: The Dialogue of Psychology with Political Science." American Political Science Review 79 (2): 293-304.

Singer, J. David. 1961. "The Level-of-Analysis Problem in International Relations." World Politics 14 (1): 77-92. 
Singer, J. David, Stuart Bremer, and John Stuckey. 1972. "Capability Distribution, Uncertainty, and Major Power War, 1820-1965." In Peace, War, and Numbers, ed. Bruce M. Russett. Beverly Hills, CA: Sage Publications.

Siverson, Randolph M. 1995. "Democracies and War Participation: In Defense of the Institutional Constraints Argument." European Journal of International Relations 1 (4): 481-489.

Slantchev, Branislav L. 2003. "The Power to Hurt: Costly Conflict with Completely Informed States." American Political Science Review 97 (1): 123-133.

Slantchev, Branislav L. 2005. "Military Coercion in Interstate Crises." American Political Science Review 99 (4): 533-547.

Slantchev, Branislav L. 2006. "Politicians, the Media, and Domestic Audience Costs." International Studies Quarterly 50 (2): 445-477.

Slantchev, Branislav L. 2010. “Feigning Weakness." International Organization 64 (3): 357-388.

Smith, Alastair. 1998. "Fighting Battles, Winning Wars." Journal of Conflict Resolution 42 (3): 301-320.

Smith, Alastair, and Allan C Stam. 2004. "Bargaining and the Nature of War." Journal of Conflict Resolution 48 (6): 783-813.

Smith, Anthony D. 2001. "Will and Sacrifice: Images of National Identity." Millennium: Journal of International Studies 30 (3): 571-584.

Smith, Hugh. 2005. "What Costs Will Democracies Bear? A Review of Popular Theories of Casualty Aversion." Armed Forces \& Society 31 (4): 487-512.

Sniehotta, Falko F., Urte Scholz, and Ralf Schwarzer. 2005. "Bridging the intention-behaviour gap: Planning, self-efficacy, and action control in the adoption and maintenance of physical exercise." Psychology \& Health 20 (2): 143-160.

Snyder, Glenn H., and Paul Diesing. 1977. Conflict Among Nations: Bargaining, Decision Making, and System Structure in International Crises. Princeton, NJ: Princeton University Press.

Snyder, Jack. 1977. The Soviet Strategic Culture: Implications for Limited Nuclear Operations. Santa Monica, CA: RAND Institute.

Snyder, Jack. 1991. Myths of Empire: Domestic Politics and International Ambition. Ithaca, NY: Cornell University Press.

Snyder, Jack, and Erica D. Borghard. 2011. "The Cost of Empty Threats: A Penny, Not a Pound." American Political Science Review 105 (August 2011): 437-456. 
Snyder, Mark, and Nancy Cantor. 1998. "Understanding Personality and Social Behavior: A Functionalist Strategy." In The Handbook of Social Psychology, ed. Daniel T. Gilbert, Susan T. Fiske, and Gardner Lindzey. 4th ed. New York: McGraw-Hill.

Sovey, Allison J., and Donald P. Green. 2011. “Instrumental Variables Estimation in Political Science: A Readers' Guide." American Journal of Political Science 55 (1): 288-200.

Spirling, Arthur. 2012. "US Treaty-making with American Indians: Institutional Change and Relative Power, 1784-1911." American Journal of Political Science 56 (1): 84-97.

Sprout, Harold, and Margaret Sprout. 1957. "Environmental factors in the study of international politics." Journal of Conflict Resolution 1 (4): 309-328.

Stam, Allan C III. 1996. Win, Lose, or Draw: Domestic Politics and the Crucible of War. Ann Arbor: University of Michigan Press.

Stanley, Elizabeth A. 2009. Paths to Peace: Domestic Coalition Shifts, War Temrination and the Korean War. Stanford: Stanford University Press.

Starr, Harvey. 1978. "'Opportunity" and "Willingness" as Ordering Concepts in the Study of War." International Interactions 4 (4): 363-387.

Stein, Arthur A. 1993. "Coordination and Collaboration: Regimes in an Anarchic World." In Neorealism and Neoliberalism: The Contemporary Debate, ed. David A. Baldwin. New York: Columbia University Press.

Streich, Philip, and Jack S. Levy. 2007. "Time Horizons, Discounting, and Intertemporal Choice." Journal of Conflict Resolution 51 (2): 199-226.

Stroud, Sarah, and Christine Tappolet. 2003. Weakness of Will and Practical Irrationality. Oxford: Clarendon Press.

Sullivan, Patricia L. 2007. "War Aims and War Outcomes." Journal of Conflict Resolution 51 (3): 496-524.

Sullivan, Patricia L. 2008. "Sustaining the Fight: A Cross-Sectional Time-Series Analysis of Public Support for Ongoing Military Interventions." Conflict Management and Peace Science 25 (2): 112-135.

Sullivan, Patricia L., and Michael T. Koch. 2009. "Military Interventions by Powerful States, 1945-2003." Journal of Peace Research 46 (5): 707-718.

Tang, Shiping. 2005. "Reputation, Cult of Reputation, and International Conflict." Security Studies 14 (1): 34-62. 
Tang, Shiping. 2008. "Fear in International Politics: Two Positions." International Studies Review 10 (3): 451-471.

Tangney, June P., Roy F. Baumeister, and Angie Luzio Boone. 2004. “High SelfControl Predicts Good Adjustment, Less Pathology, Better Grades, and Interpersonal Success." Journal of Personality 72 (2): 273-324.

Terracciano, A., A. M. Abdel-Khalek, N. Ádám, L. Adamovová, C.-k. Ahn, H.-n. Ahn, B. M. Alansari, L. Alcalay, J. Allik, A. Angleitner, M. D. Avia, L. E. Ayearst, C. Barbaranelli, A. Beer, M. A. Borg-Cunen, D. Bratko, M. Brunner-Sciarra, L. Budzinski, N. Camart, D. Dahourou, F. De Fruyt, M. P. de Lima, G. E. H. del Pilar, E. Diener, R. Falzon, K. Fernando, E. Ficková, R. Fischer, C. Flores-Mendoza, M. A. Ghayur, S. Gülgöz, B. Hagberg, J. Halberstadt, M. S. Halim, M. Hřebíčková, J. Humrichouse, H. H. Jensen, D. D. Jocic, F. H. Jónsson, B. Khoury, W. Klinkosz, G. Knežević, M. A. Lauri, N. Leibovich, T. A. Martin, I. Marušić, K. A. Mastor, D. Matsumoto, M. McRorie, B. Meshcheriakov, E. L. Mortensen, M. Munyae, J. Nagy, K. Nakazato, F. Nansubuga, S. Oishi, A. O. Ojedokun, F. Ostendorf, D. L. Paulhus, S. Pelevin, J.-M. Petot, N. Podobnik, J. L. Porrata, V. S. Pramila, G. Prentice, A. Realo, N. Reátegui, J.-P. Rolland, J. Rossier, W. Ruch, V. S. Rus, M. L. Sánchez-Bernardos, V. Schmidt, S. Sciculna-Calleja, A. Sekowski, J. ShakespeareFinch, Y. Shimonaka, F. Simonetti, T. Sineshaw, J. Siuta, P. B. Smith, P. D. Trapnell, K. K. Trobst, L. Wang, M. Yik, A. Zupančič, and R. R. McCrae. 2005. “National Character Does Not Reflect Mean Personality Trait Levels in 49 Cultures." Science 310 (5745): 96-100.

Tetlock, Philip E. 2003. "Thinking the unthinkable: sacred values and taboo cognitions." Trends in Cognitive Sciences 7 (7): 320-324.

Tett, Robert P., and Dawn D. Burnett. 2003. “A Personality Trait-Based Interactionist Model of Job Performance." Journal Of Applied Psychology 88 (3): 500-517.

Tett, Robert P., and Hal A. Gutterman. 2000. "Situation trait relevance, trait expression, and cross-situational consistency: Testing a principle of trait activation." Journal of Research in Personality 36 (4): 397-423.

Thaler, Richard. 1985. "Mental Accounting and Consumer Choice." Marketing Science 4 (3): 199-214.

Thaler, Richard, and H.M. Shefrin. 1981. "An Economic Theory of Self-Control." Journal of Political Economy 89 (2): 392-406.

Thompson, William R. 2003. "A Streetcar Named Sarajevo: Catalysts, Multiple Causation Chains, and Rivalry Structures." International Studies Quarterly 47 (3): 453-474.

Thucydides. 1998. The Landmark Thucydides: A Comprehensive Guide to the Peloponnesian War. Trans. Richard Crawley and Ed. Robert B. Strassler. New York: Simon \& Schuster. 
Tice, Dianne M., Roy F. Baumeister, Dikla Shmueli, and Mark Muraven. 2007. "Restoring the self: Positive affect helps improve self-regulation following ego depletion." Journal of Experimental Social Psychology 43 (3): 379-384.

Tingley, Dustin H., and Barbara F. Walter. 2011. “The Effect of Repeated Play on Reputation Building: An Experimental Approach." International Organization 65 (2): 343-365.

Tingley, Dustin, Teppei Yamamoto, Luke Keele, and Kosuke Imai. 2012. "mediation 4.0: Statistical Analysis of Causal Mechanisms Using R." Journal of Statistical Software Forthcoming.

Tittle, Charles R., David A. Ward, and Harold G. Grasmick. 2003. "Self-control and Crime/Deviance: Cognitive vs. Behavioral Measures." Journal of Quantitative Criminology 19 (4): 333-365.

Tittle, Charles R., David A. Ward, and Harold G. Grasmick. 2004. "Capacity for SelfControl and Individuals' Interest in Exercising Self-Control." Journal of Quantitative Criminology 20 (2): 143-172.

Tobin, Henry, and A.W. Logue. 1994. "Self-Control Across Species (Columba livia, Homo sapiens, and Rattus norvegicus)." Journal of Comparative Psychology 108 (2): 126-133.

Todd, Peter M., and Gerd Gigerenzer. 2003. "Bounding rationality to the world." Journal of Economic Psychology 24 (2): 143-165.

Toft, Monica Duffy. 2006. "Issue Indivisibility and Time Horizons as Rationalist Explanations for War." Security Studies 15 (1): 34-69.

Tomz, Michael. 2007. "Domestic Audience Costs in International Relations: An Experimental Approach." International Organization 61 (4): 821-40.

Trager, Robert F. 2011. "Multidimensional Diplomacy." International Organization 65 (3): 469-506.

Trager, Robert F., and Lynn Vavreck. 2011. "The Political Costs of Crisis Bargaining: Presidential Rhetoric and the Role of Party." American Journal of Political Science 55 (3): 526-545.

Travers, T.H.E. 1979. “Technology, Tactics, and Morale: Jean de Bloch, the Boer War, and British Military Theory, 1900-1914." The Journal of Modern History 51 (2): 264286.

Treier, Shawn, and Simon Jackman. 2008. "Democracy as a Latent Variable." American Journal of Political Science 52 (1): 201-217. 
Trivedi, Ranak B., Hayden B. Bosworth, and George L. Jackson. 2011. "Resilience in Chronic Illness." In Resilience in Aging: Concepts, Research, and Outcomes, ed. Barbara Resnick, Lisa P. Gwyther, and Karen A. Roberto. Springer.

Trope, Yaacov, and Nira Liberman. 2003. "Temporal Construal." Psychological Review 110 (3): 403-421.

Turner, Frederick Jackson. 1956. "The Significance of the Frontier in American History." In The Turner Thesis: Concerning the Role of the Frontier in American History, ed. George Rogers Taylor. Boston: D.C. Heath and Company.

Twomey, Christopher P. 2008. "Lacunae in the Study of Culture in International Security." Contemporary Security Policy 29 (2): 338-357.

Udehn, Lars. 2001. Methodological Individualism: Background, history and meaning. London: Routledge.

Ungar, Michael. 2004. "A Constructionist Discourse on Resilience: Multiple Contexts, Multiple Realities among At-Risk Children and Youth." Youth \& Society 35 (3): 341-365.

Valentino, Benjamin A., Paul K. Huth, and Sarah E. Croco. 2010. “Bear Any Burden? How Democracies Minimize the Costs of War." Journal of Politics 72 (2): 528-544.

Van de Vliert, Evert, Huadong Yang, Yongli Wang, and Xiao-peng Ren. 2013. "Climato-Economic Imprints on Chinese Collectivism." Journal Of Cross-Cultural Psychology 44 (4): 589-605.

Van Evera, Stephen. 1984. "The Cult of the Offensive and the Origins of the First World War." International Security 9 (1): 58-107.

Van Evera, Steven. 1999. Causes of War: Power and the Roots of Conflict. Ithaca: Cornell University Press.

Vasquez, Joseph Paul III. 2005. "Shouldering the Soldiering : Democracy, Conscription, and Military Casualties." Journal of Conflict Resolution 49 (6): 849-873.

Velleman, J. David. 2007. "What Good is a Will?" In Action in Context, ed. Anton Leist. Berlin: Walter de Gruyter.

Viscusi, W. Kip, and Joseph E. Aldy. 2003. "The Value of a Statistical Life: A Critical Review of Market Estimates Throughout the World." Journal of Risk and Uncertainty 27 (1): 5-76.

Voeten, Erik. 2000. "Clashes in the Assembly." International Organization 54 (2): 185215.

von Neumann, John, and Oskar Morgenstern. 1944. Theory of Games and Economic Behavior. Princeton: Princeton University Press. 
Wagner, R. Harrison. 2000. "Bargaining and War." American Journal of Political Science 44 (3): 469-484.

Walker, Stephen G., Mark Schafer, and Michael D. Young. 1999. "Presidential operational codes and foreign policy conflicts in the post-cold war world." Journal of Conflict Resolution 43 (5): 610-625.

Wallerstein, Immanuel. 1974. The Modern World-System I: Cpaitalist Agriculture and the Origins of the European World-Economy in the Sixteenth Century. New York: Academic Press.

Walt, Stephen M. 1985. "Alliance Formation and the Balance of World Power." International Security 9 (4): 3-43. The MIT Press

Walt, Stephen M. 1999. “Rigor or Rigor Mortis?: Rational Choice and Security Studies." International Security 23 (4): 5-48. The MIT Press

Walter, Barbara F. 1997. "The Critical Barrier to Civil War Settlement." International Organization 51 (03): 335-364.

Walter, Barbara F. 2006. "Building Reputation: Why Governments Fight Some Separatists but Not Others." American Journal of Political Science 50 (2): 313-330.

Waltz, Kenneth N. 1959. Man, The State, and War: A Theoretical Analysis. New York: Columbia University Press.

Waltz, Kenneth N. 1979. Theory of International Politics. Boston: McGraw-Hill.

Wang, Mei, Marc Oliver Rieger, and Thorsten Hens. 2010. How Time Preferences Differ: Evidence from 45 Countries. Technical report Swiss Finance Institute Research Paper No. 09-47.

Wansbeek, Tom, and Erik Meijer. 2000. Measurement Error and Latent Variables in Econometrics. Amsterdam: North-Holland.

Warren, Michael. 2011. "Paul Ryan Embraces American Exceptionalism, Rejects Isolationism in Foreign Policy Speech." In The Weekly Standard. http://www.weeklystandard.com/blogs/ ryan-embraces-exceptionalism-rejects-isolationism-foreign-policy-speech_ 573194.html.

Watson, Alexander. 2008. Enduring the Great War: Combat, Morale and Collapse in the German and British Armies, 1914-1918. Cambridge University Press.

Watson, Carl B., Martin M. Chemers, and Natalya Preiser. 2001. "Collective Efficacy: A Multilevel Analysis." Personality and Social Psychology Bulletin 27 (8): 1057-1068. 
Weber, Elke U., Ann-Renée Blais, and Nancy E. Betz. 2002. "A Domain-specific Risk-attitude Scale: Measuring Risk Perceptions and Risk Behaviors." Journal Of Behavioral Decision Making 15 (4): 263-290.

Weber, Elke U., and Christopher K. Hsee. 1998. “What Folklore Tells Us about Risk and Risk Taking: Cross-Cultural Comparisons of American, German, and Chinese Proverbs." Organizational Behavior and Human Decision Processes 75 (2): 170186.

Weber, Max. 1904/1984. The Protestant Ethic and the Spirit of Capitalism. Winchester, MA: Allen \& Unwin.

Wedeen, Lisa. 2002. "Conceptualizing Culture: Possibilities for Political Science." American Political Science Review 96 (4): 713-28.

Weeks, Jessica L. 2008. “Autocratic Audience Costs: Regime Type and Signaling Resolve." International Organization 62 (1): 35-64.

Weidmann, Nils B., Doreen Kuse, and Kristian Skrede Gleditsch. 2010. “The Geography of the International System: The CShapes Dataset." International Interactions 36 (1): 86-106.

Wendt, Alexander. 1987. "The Agent-Structure Problem in International Relations Theory." International Organization 41 (3): 335-69.

Wendt, Alexander. 1992. "Anarchy is what States Make of it: the Social Construction of Power Politics." International Organization 46 (2): 391-425.

Wendt, Alexander. 1998. "On constitution and causation in International Relations." Review of International Studies 24 (5): 101-117.

Wendt, Alexander. 1999. Social Theory of International Politics. Cambridge, UK: Cambridge University Press.

Wendt, Alexander. 2004. "The state as person in international theory." Review of International Studies 30 (2): 289-316.

White, Jeffrey. 2011. The Grinding War in Libya Favors Qadhafi. Technical report The Washington Institute for Near East Policy.

Whiteside, Stephen P., and Donald R. Lynam. 2001. "The Five Factor Model and impulsivity: using a structural model of personality to understand impulsivity." Personality and Individual Differences 30 (4): 669-689.

Wight, Colin. 2004. "State agency: social action without human activity?" Review of International Studies 30 (2): 269-280. 
Wikstrom, Per-Olof H., and Kyle Treiber. 2007. "The Role of Self-Control in Crime Causation: Beyond Gottfredson and Hirschi's General Theory of Crime." European Journal of Criminology 4 (2): 237-264.

Williams, Michael C. 2008. Culture and Security: Symbolic Power and the Politics of International Security. London: Routledge.

Winship, Christopher, and Larry Radbill. 1994. "Sampling Weights and Regression Analysis." Sociological Methods E Research 23 (2): 230-257.

Wittkopf, Eugene R. 1990. Faces of internationalism: Public Opinion and American foreign policy. Duke University Press.

Wolfers, Arnold. 1962. Discord and Collaboration: Essays on International Politics. Baltimore: Johns Hopkins University Press.

Wolford, Scott. 2007. “The Turnover Trap: New Leaders, Reputation, and International Conflict." American Journal of Political Science 51 (4): 772-788.

World Values Survey Association. 2009. “World Values Survey 1981-2008 Official Aggregate v. 20090901." http://www.wvsevsdb.com.

Wright, Quincy. 1964. A Study of War. Abridged ed. Chicago: University of Chicago Press.

Xiang, Jun. 2010. "Relevance as a Latent Variable in Dyadic Analysis of Conflict." Journal of Politics 72 (2): 484-498.

Yglesias, Matthew. 2006. “The Green Lantern Theory of Geopolitics.” In TPM Cafe. http://www. smirkingchimp.com/thread/845.

Young, Joseph K. 2008. "Repression, Dissent, and the Onset of Civil War." PhD Dissertation, Florida State University.

Young, Oran R. 1968. The Politics of Force: Bargaining During International Crises. Princeton, NJ: Princeton University Press.

Zakaria, Fareed. 2008. The Post-American World. New York: W.W. Norton.

Zaller, John R. 1992. The Nature and Origins of Mass Public Opinion. Cambridge: Cambridge University Press. 